\title{
Hysteresis and Capacitive Features of Perovskite Solar Cells
}

Hysterese und Kapazitive Eigenschaften von Perowskit-Solarzellen
Histéresis y Características Capacitivas

de Celdas Solares de Perovskitas

\section{Doctoral Thesis}

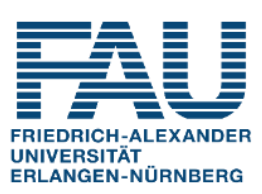

Dissertation zur Erlangung der

Doktorgrades Dr.-Ing.

in der Technischen Fakultät der

Friedrich-Alexander Universität

Erlangen-Nürnberg, Deutschland (FAU), in einem binationalen programm mit der Jaume I Universität in Castelló, Spanien, vorgelegt von

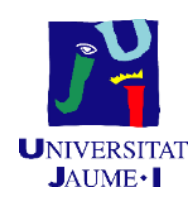

Memoria para optar al grado de

Doctor en Ciencias

de la Escuela de Doctorado de la

Universitat Jaume I de Castelló, España (UJI), en régimen de co-tutela con la Universidad Friedrich-Alexander de

Erlangen-Núremberg, Alemania, presentada por:

\section{Osbel Almora Rodríguez}

aus Pinar del Río, Kuba, und betreut an der FAU durch:

Prof. Dr. Christoph J. Brabec de Pinar del Río, Cuba, y supervisada en la UJI por: 

Osbel Almora

\section{Hysteresis and Capacitive Features of Perovskite Solar Cells}

\section{Erlangen}

2020 
Als Dissertation genehmigt in der

Doktoratsprogramm in

Werkstoffwissenschaften von der

Technischen Fakultät der

Friedrich-Alexander Universität

Erlangen-Nürnberg, Deutschland

Ort and Datum der mündlichen Prüfung:
Aprobado como disertación

en el Programa de

Doctorado en Ciencias

de la Escuela de Doctorado de la

Universitat Jaume I

de Castelló, España

Lugar y Fecha del examen oral:

Fundings

- Generalitat Valenciana grant GRISOLIA/2014/035.

- VDI/VD Innovation + Technik GmbH (Project-title: PV-ZUM)

- Erlangen Graduate School of Advanced Optical Technologies (SAOT) funded by the German Research Foundation in the framework of the German Excellence Initiative. 


\section{Acknowledgments}

Firstly, I would like to thank all the colleagues who provided and/or assisted the fabrication of the samples for the several experiments discussed in this work. All my gratitude to Clara Aranda, Elena Mas Marza, Antonio Guerrero, Isaac Zarazúa, Kyung Taek Cho, Iwan Zimmermann, Yi Hou, Wei Meng, Marta Valles Pelarda, Marisé García Batlle and Yicheng Zhao.

Many thanks for the contributions in terms of numerical simulations by Pilar López Varo and Prof. Juan Antonio Jiménez Tejada, the assistance of Daniel Miravet with Mathematica coding and the first principles calculations by Carlos Echeverría Arrondo. The contributions of Alfredo Gonzalez Lezcano on the hopping model are also acknowledged.

I would also like to extend my thanks to Francisco Fabregat Santiago and Gebhard J. Matt for their support as group leaders and assistance/instruction with instrumentation tasks. Furthermore, the many assistances provided by Thomas Heumüller and his incomes with the transient photovoltage technique were greatly appreciated.

I would like to acknowledge Prof. Juan Bisquert for his support and many inspiring lessons, and Prof. Mohammad Khaja Nazeeruddin for the opportunity to make a research stay at EPFL Valais Wallis, Sion, Switzerland.

I wish to express my sincere and deepest appreciation to my supervisors, Prof. Germà Garcia Belmonte and Prof. Christoph J. Brabec. Every moment under their guidance has been my privilege.

And last but not least, I want to thank all my colleagues, technicians, and staff at the Institute of Advanced Materials, Castellón, Spain, and the Institute of Materials for Electronics and Energy Technologies, Erlangen, Germany, thanks to my friends and my family, for all the support, lessons and good moments during these years. 


\section{Abstract}

In the present work, anomalous distortions occurring in the current-voltage characteristic of perovskite solar cells (PSCs), usually called $J-V$ curve hysteresis, are studied by several methods. This includes dynamic direct current (DC) mode $J-V$ experiments and impedance spectroscopy (IS) analyses in dark and under illumination. Initially, the $\mathrm{CH}_{3} \mathrm{NH}_{3} \mathrm{PbI}_{3}$ absorber material is characterized by alternating current (AC) and transient techniques showing ionic-related features. Subsequently, dark $J-V$ curves of PSCs measured under different conditions are shown to exhibit capacitive hysteretic currents. This is related with low frequency excess capacitance in the dark IS spectra. These two features are correlated with the response of mobile ions in space charge regions close to the interfaces. The ion-related low frequency capacitance is shown to hinder the evaluation of deep trap and shallow doping concentrations from IS analyses as a function of temperature and DC bias, i.e. TAS and Mott-Schottky analysis, respectively. The light $J-V$ curve hysteresis was checked at faster scan rates after pre-bias in different device structures. The results were simulated by drift diffusion methods, suggesting that the formation of ionic dipoles can create large hysteresis. The light IS analyses at open-circuit allowed to identify different recombination mechanisms via ideality factor parametrization and revealed different exponential trends for the low-frequency capacitance. The low frequency capacitance was also studied at short-circuit under light and forward bias in the dark. The large values of capacitance in the sub-Hz regime were explained in terms of mobile ions space charges and chemical capacitances assuming a proportionality between the number of ionized/activated mobile ions and the concentration of charge carriers and photon fluence. Finally, a new method of characterization of photo-sensitive devices was introduced, named light intensity modulated impedance spectroscopy (LIMIS). This is based on the evaluation of photo-impedance from both, the individual photovoltage and photocurrent signals, under small AC light perturbation at DC open circuit. The impedance difference between IS and LIMIS informs on recombination velocity in traditional photovoltaics. Preliminary measurements of LIMIS in PSCs reveal significant impedance differences as light intensity increases and provide improved measurements of charge carrier lifetimes. 


\section{Zusammenfassung}

In der vorliegenden Arbeit werden anomale Verzerrungen in der Strom-SpannungsCharakteristik von Perowskit-Solarzellen (PSCs), die als J-V-Kurven-Hysterese bezeichnet werden, im Dunkeln und unter Beleuchtung mit verschiedenen Methoden untersucht. Dazu gehören J-V-Experimente im dynamischen Gleichstrommodus (DC) und Impedanzspektroskopieanalysen (IS). Anschließend werden J-V-Dunkelkurven von PSCs unter verschiedenen Bedingungen gemessen, die kapazitive Hystereseströme aufweisen, welche mit niederfrequenten Kapazitätsüberhöhungen in den Dunkel-IS-Spektren zusammenhängen. Diese beiden Merkmale sind mit der Modulation mobiler Ionen in Raumladungszonen an der Grenzflächen korreliert. Es wird gezeigt, dass die ionenbezogene Niederfrequenzkapazität die Auswertung von tiefen Fallen- und flachen Dotierstoffkonzentrationen aus IS-Analysen als Funktion der Temperatur, genannt Wärmeleitfähigkeitsspektroskopie, und als Funktion der Gleichstromvorspannung, bekannt als Mott-Schottky-Plot, behindert. Die leichte J-V-KurvenHysterese wurde bei schnelleren Abtastraten nach Vorspannung in verschiedenen Bauelementstrukturen überprüft. Die Ergebnisse wurden durch Drift-Diffusionsmethoden simuliert, was darauf hindeutet, dass die Bildung von Ionendipolen eine große Hysterese erzeugen kann. Die Licht-IS-Analysen bei offenem Stromkreis ermöglichten die Identifizierung verschiedener Rekombinationsmechanismen durch Idealitätsfaktor-Parametrisierung und zeigten unterschiedliche exponentielle Trends für die niederfrequente Kapazität. Die niederfrequente Kapazität wurde auch bei Kurzschluss unter Licht und bei Vorwärtsspannung im Dunkeln untersucht. Die großen Kapazitätswerte im Sub-Hz-Regime wurden anhand der Raumladungen mobiler Ionen und der chemischen Kapazitäten erklärt, wobei eine Proportionalität zwischen der Anzahl ionisierter/aktivierter mobiler Ionen und der Konzentration von Ladungsträgern und der Photonenfluenz angenommen wurde. Schließlich wurde eine neue Methode zur Charakterisierung lichtempfindlicher Bauelemente eingeführt, die als Lichtintensitätsmodulierte Impedanzspektroskopie (LIMIS) bezeichnet wird und auf der Auswertung der Photoimpedanz sowohl der einzelnen Photospannungs- als auch der Photostromsignale bei kleinen AC-Störungen der Beleuchtung unter DC-Leerlaufspannung basiert. Die Impedanzdifferenz zwischen IS und LIMIS gibt Aufschluss über die Rekombinationsgeschwindigkeit in der traditionellen Photovoltaik. Vorläufige Messungen von LIMIS in PSCs zeigen signifikante Impedanzunterschiede mit zunehmender Lichtintensität und liefern verbesserte Messungen der Ladungsträger-Lebensdauer. 


\section{Resumen}

En el presente trabajo se estudian por varios métodos las distorsiones anómalas en la característica de corriente-voltaje de las celdas solares de perovskita (PSC), típicamente llamada histéresis de $J-V$. Esto incluye experimentos dinámicos de $J-V$ en modo de corriente continua (DC) y análisis de espectroscopía de impedancia (IS) en oscuridad y bajo iluminación. Las curvas $J-V$ en oscuridad de las PSCs exhiben corrientes capacitivas, relacionadas con un exceso de capacitancia de baja frecuencia en los espectros de IS. Estas dos características están correlacionadas con la respuesta de iones móviles en regiones espaciales de carga hacia las interfaces. Es mostrado que la capacitancia de baja frecuencia relacionada con iones móviles dificulta la evaluación de las concentraciones de trampas de niveles profunda y de dopaje de los análisis de IS en función de la temperatura (TAS), y el voltaje DC (análisis de Mott-Schottky). La histéresis de la curva $J-V$ bajo iluminación se midió a velocidades de barrido rápidas después de pre-polarización en diferentes estructuras de PSCs. Los resultados fueron simulados por métodos numéricos, sugiriendo que la formación de dipolos iónicos puede crear gran histéresis. Los análisis de IS bajo iluminación en circuito abierto permitieron identificar diferentes mecanismos de recombinación a través de la parametrización del factor de idealidad y revelaron diferentes tendencias exponenciales para la capacitancia de baja frecuencia. Esta capacitancia también se estudió en cortocircuito y bajo polarización directa en oscuridad. Los grandes valores de capacitancia por debajo de las unidades de $\mathrm{Hz}$ se explicaron en términos de regiones de cargas espaciales de iones móviles y capacitancias químicas, suponiendo una proporcionalidad entre el número de iones móviles ionizados/activados y la concentración de portadores de carga y flujo de fotones. Finalmente, se introdujo un nuevo método de caracterización de dispositivos fotosensibles, denominado espectroscopía de impedancia por intensidad de luz modulada (LIMIS) basado en la evaluación de la foto-impedancia bajo una pequeña perturbación de la luz AC de las señales individuales de foto-voltaje y fotocorriente en circuito abierto DC. La diferencia de impedancia entre IS y LIMIS informa de la velocidad de recombinación en dispositivos fotovoltaicos tradicionales. Las mediciones preliminares de LIMIS en PSC informan de diferencias significativas en impedancia a medida que la intensidad de la luz crece y aporta una corrección a la evaluación de los tiempos característicos. 


\section{Table of Contents}

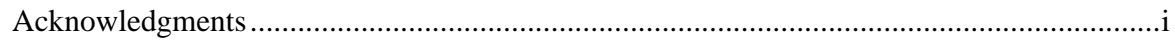

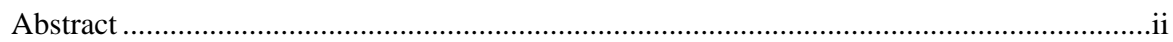

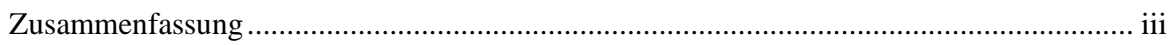

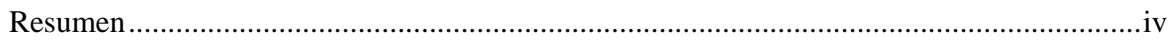

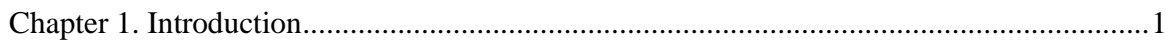

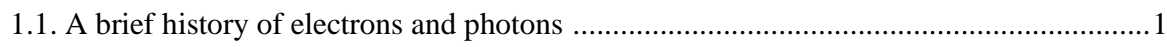

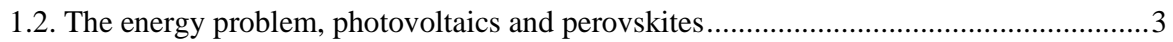

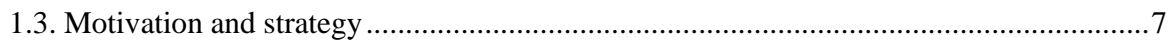

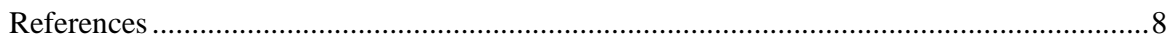

Chapter 2. The physics of solar cells, perovskites and capacitance ………………...........13

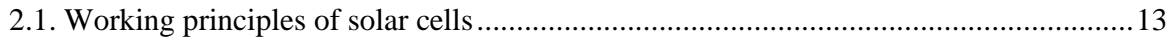

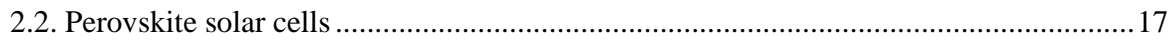

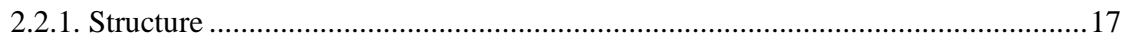

2.2.2. Materials

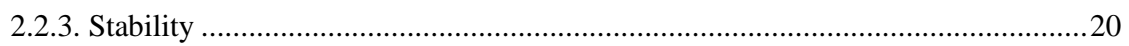

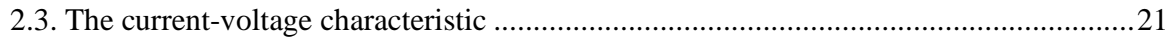

2.4. Phenomenology of the hysteresis in perovskite solar cells ............................................29

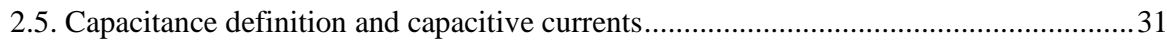

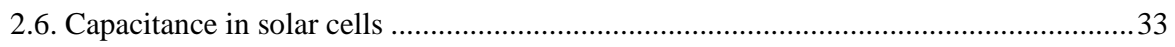

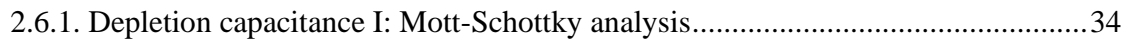

2.6.2. Depletion capacitance II: Deep trap levels ............................................................ 38

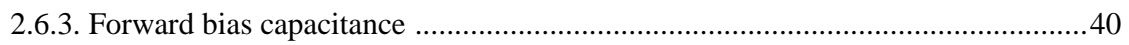

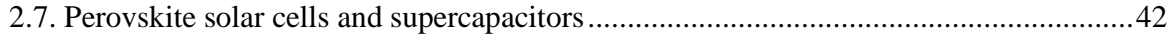

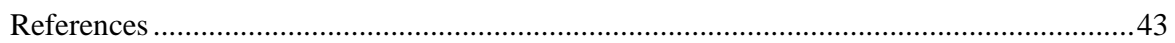

Chapter 3. Characterization techniques: concepts and instrumentation...............................49

3.1. Structure and materials of the characterized samples ...................................................49

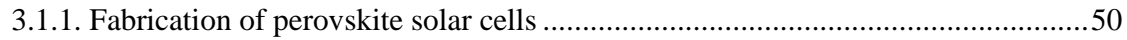

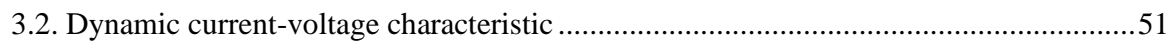

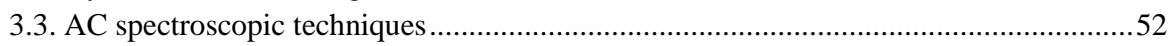

3.3.1. Potentiostatic impedance spectroscopy............................................................52

3.3.2. Mott-Schottky measurement ..........................................................................57

3.3.3. Thermal admittance spectroscopy (TAS) measurement ......................................59 
3.3.4. Light modulated photocurrent and photovoltage spectroscopies .61

3.3.5. Instrumentation of impedance and light modulating spectroscopies ..................63

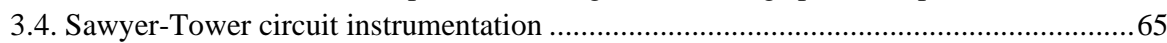

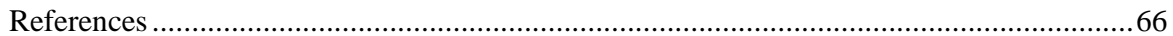

Chapter 4. Dark characterizations and modeling ................................................................69

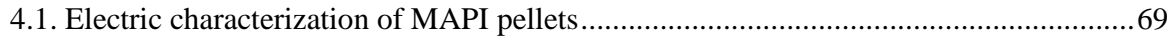

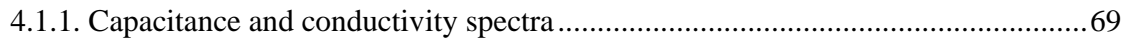

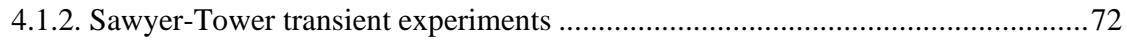

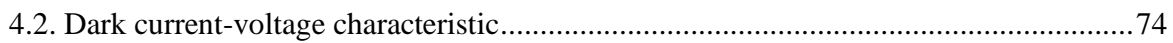

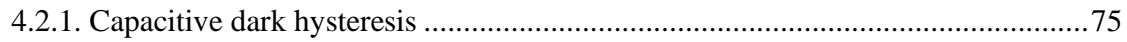

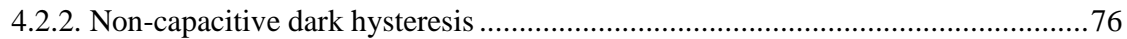

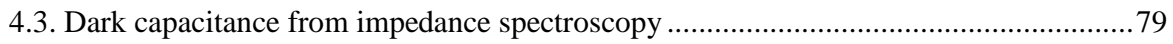

4.3.1. Thermal admittance spectroscopy .................................................................... 80

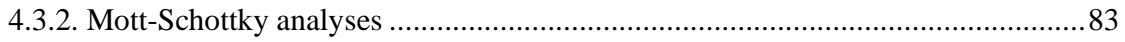

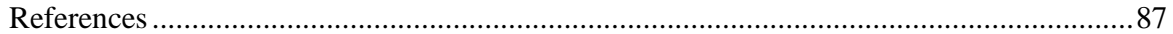

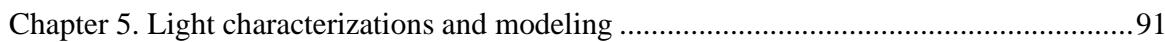

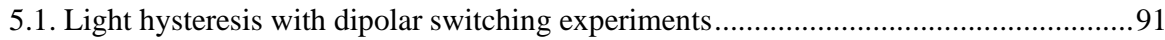

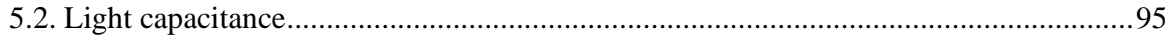

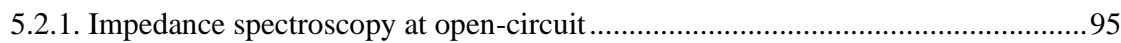

5.2.2. Ionization, space charge regions and chemical capacitances ..............................99

5.3. Light intensity modulated impedance spectroscopy (LIMIS) ........................................ 103

5.3.1. Theoretical introduction to LIMIS: the one-sided p-n junction case ...................104

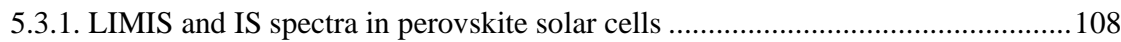

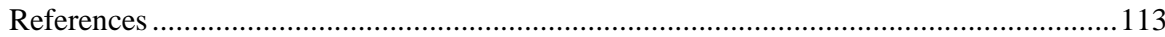

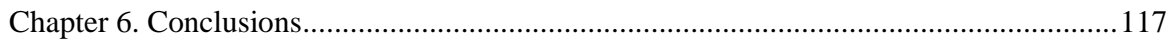

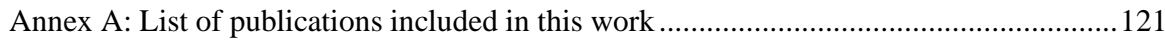

Annex B: List of other publications by the author..........................................................122 


\section{Chapter 1. Introduction}

Science and technology can tell the history of humankind and one could even say that they write the history of the human need to overcome the continuously evolving life challenges. In terms of needs, the energy has always been a constant key element since more than 50 thousand years ago, when heat and light were first necessary to survive weather and predators, until modern times.

The energy is the quantitative property of matter and radiation which manifests as a capacity to perform work, such as causing motion or the interaction of molecules. But, for an average daily worker in modern societies the meaning of energy is probably more associated with electricity, which holds/assists most of transportation to/from/as work, work itself and home/leisure activities. The electricity production can be performed in several ways, being the photovoltaic (PV) solar cells one of the most environment-friendly and sustainable technologies there are.

The PV devices transform light energy into electric energy, to be used as electricity for satisfying a given demand. The present work summarizes some recent contributions by the author in the research field of solar cells. Particularly, the focus of this text is set on perovskite solar cells and the understanding of their optoelectronic characteristics.

In this introductory chapter a first section intends to provide some context by introducing necessary notions and concepts, following a chronological order and highlight-ting main history contributors. In a second section the context of the general problem and solution strategies are provided: the development of perovskite-absorber photovoltaic solar cells. This is a renewable energy strategic response to the problem of the transition to a sustainable and clean energy generation scheme, at global scale. Later, in a third section, the specific problems attended in this work are introduced and the general chapters structure is commented.

\subsection{A brief history of electrons and photons}

A necessary start point would be the "age of enlightenment", between the end of the $17^{\text {th }}$ century and the next one, ${ }^{[1]}$ with I. Newton, marking a singular milestone with his Principia, ${ }^{[2]}$ the pillar of the classical mechanics, and Opticks, ${ }^{[3]}$ the foundation of the physical optics. Newton, independently to G.W. Leibniz, ${ }^{[4]}$ also invented the differential and integral calculus. The development of this mathematical branch catalyzed the progress of physics, and by nearly half a century later L. Euler ${ }^{[5]}$ had published the set of differential equations named after him, including the general form of the continuity equation.

Simultaneously, from mid- $18^{\text {th }}$ century on, the electricity raised as a major field from the studies of B. Franklin ${ }^{[6]}$ C.-A. Coulomb,${ }^{[7]}$ L. Galvani ${ }^{[8]}$ and A. Volta. ${ }^{[9]}$ Then, Franklin realized the effect of dielectric charge storing in the first capacitor, the Leyden jar, ${ }^{[10]}$ and Volta introduced the denomination of condenser.

In the first decades of the $19^{\text {th }}$ century, the electromagnetism was introduced by H.C. Oersted $^{[11]}$ and A.-M. Ampère. ${ }^{[12]}$ Purposely, S.D. Poisson ${ }^{[13]}$ had derived his equation relating the potential and the charge density. Then, M. Faraday ${ }^{[14]}$ discovered the electromagnetic induction, established the basis for the concept of the electromagnetic field and additionally he likewise first documented semiconducting behavior in 1833. ${ }^{[15]}$ 
Born and doctored in Erlangen, G.S. Ohm ${ }^{[16]}$ presented his law relating the voltage $V$ and the current density $J$ due to electric field $\xi$ drift, and the useful concept of electrical resistance $R$. In 1839 A.-E. Becquerel first observed photovoltaic effect. ${ }^{[15,17]}$ Later, G.R. Kirchhoff ${ }^{[18]}$ postulated his circuit laws and, by the way, the black-body radiation concept. Then, "there was light" with the equations of electromagnetism from J.C. Maxwell, ${ }^{[19]}$ which were subsequently reformulated by $\mathrm{O}$. Heaviside ${ }^{[20]}$ in terms of electric and magnetic forces and energy flux. Additionally, Heaviside also introduced the theory of the complex numbers for the description of electrical circuits, and a valuable terminology including impedance $Z$, admittance $Y$, capacitance $C$, and inductance $L$. But it was H.R. Hertz ${ }^{[21]}$ who experimentally proved Maxwell's electromagnetic theory of light, and furthermore he also first observed the photoelectric effect. Years later, in 1897, the electron was finally discovered by J.J. Thomson ${ }^{[22]}$ who provided the first estimates of the elementary charge $q$ and mass $m_{e}$.

In 1900 the energy quanta was introduced by M. Planck ${ }^{[23]}$ in his solution to the black-body radiation spectra. In the next year O.W. Richardson ${ }^{[24]}$ published his law on thermionic emission and the light quanta came later in 1905, the annus mirabilis of A. Einstein, ${ }^{[25]}$ when he explained the photoelectric effect and defined what we now call photons. Actually, in that year next paper ${ }^{[26]}$ the Einstein relation for the diffusion coefficient $D$ came out along with his approach to the Brownian motion.

By the 1930s E. Fermi ${ }^{[27]}$ had published his statistics and the quantum mechanics theory was already very popular. Hence F. Bloch ${ }^{[28]}$ introduced it for describing electrons in crystal lattices and A.H. Wilson ${ }^{[29]}$ came with the band theory of semiconductors. Later, important contributions to the understanding of semiconducting junctions and rectifying behaviors arose from W.H. Schottky ${ }^{[30-31]}$ and N.F. Mott. ${ }^{[32-33]}$ Nearly a decade afterwards, W.B. Shockley, ${ }^{[34-35]}$ head of the solid-state research group at Bell Labs, presented his theory on p-n junctions in semiconductors and transistors. Collaborating with W.T. Read Jr., ${ }^{[36]}$ and simultaneously to R.N. Hall, ${ }^{[37]}$ Shockley also co-credited the analysis of the non-radiative carrier recombination in semiconductors.

In 1954 D.M. Chapin, C.S. Fuller and G.L. Pearson, also at Bell Labs, announced the first practical silicon $(\mathrm{Si})$ photovoltaic cell with around $6 \%$ power conversion efficiency. ${ }^{[17,38]}$ The new application also interested Shockley, thus he and H.-J. Queisser first calculated the detailed balance limit of efficiency for $\mathrm{p}$-n single junction solar cells. ${ }^{[39]}$

From the 1960s on, the first generation of Si-based photovoltaics (SiSCs) have led the market and it is still among the most successful devices in terms of best research-cell efficiencies. ${ }^{\text {[0-41] }}$ In the 1970 s a second generation of thin film devices emerged with an irregular presence in the market, typically amorphous-silicon (a-Si), copper-indium-gallium-selenide (CIGS) and cadmium telluride (CdTe) solar cells. ${ }^{[42]}$ Alternative technologies with specific applications or market opportunities gained attention in the late $1980 \mathrm{~s}$, like gallium arsenide $(\mathrm{GaAs}),{ }^{[43]}$ the multijunction cells ${ }^{[44]}$ and the dye sensitized solar cells (DSSCs). ${ }^{[45]}$ Similarly in the 2000s there were other emerging technologies like the organic photovoltaics (OSCs) ${ }^{[46]}$ and the inorganic kesterites CZTS. ${ }^{[47]}$ In the 2010s decade the quantum dots cells ${ }^{[48]}$ were introduced and then, the perovskite solar cells (PSCs). ${ }^{[49]}$ 


\subsection{The energy problem, photovoltaics and perovskites}

In the previous section, important moments between the $18^{\text {th }}$ century and the first half of the $20^{\text {th }}$ century were surveyed because of its importance in the theoretical framework of the following chapters. Their repercussions conditioned most of the aspects in science, technology, economy and society, as we know it. Overall, in order to continue reading, general notions on what are electrons, photons, electric current, voltage and capacitance are advised.

The attention from the 1950 s on, in the previous section, was particularly set on the development of the photovoltaic technologies (PVs), whose objective is to provide energy for electricity consumption. The difficulty to access energy resources currently in some developing countries and worldwide in a near future, known as the energy problem, is one of the top challenges towards the end of the $21^{\text {th }}$ century. ${ }^{[50-55]}$

Fossil and mineral fuels are currently over $80 \%$ of the world primary energy supply. ${ }^{[56]}$ These are non-renewal energetic sources, meaning that they will eventually reach their irreversible consumption limit. ${ }^{[57]}$ The speed at which these non-renewal resources are consumed is fostered by the increase of both population and energy usage per capita. ${ }^{[54,58]}$ But more urgent than the expiration of fossil and mineral fuels, there is the growing environmental harm as a result of such global energetic scheme. ${ }^{[59]}$ The consequent global warming is actually considered one of the biggest threats facing humankind today. ${ }^{[51,55]}$ In that scenario, the proper transition to renewal energy technologies can be the key for future sustainable societies with comparable or better than our current standards of livings. ${ }^{[57,60]}$

The ecological and economic need for renewable energies lead us to their concept, which lies in the exploitation of renewable resources that are naturally replenished on a human timescale. ${ }^{[61]}$ Typical examples are wind, tidal, biomass, and solar renewal energy. The latter is the focus of our attention here.

The solar intensity at the surface of Earth can be approached as the standard 1 sun $=$ $100 \mathrm{~mW} \cdot \mathrm{cm}^{-2},{ }^{[62]}$ and considering a $70 \%$ of sunshine days per year at 8 hours of daylight on average, the energy that Earth receives from the Sun is around $2.1 \mathrm{MW} \mathrm{h} \mathrm{m}^{-2}\left(\sim 10^{24} \mathrm{~J}\right)$ per year. ${ }^{[63]}$ On the other hand, the annual world energy consumption by 2040 is estimated to be around $2.2 \cdot 10^{11} \mathrm{MW} \mathrm{h}\left(\sim 10^{20} \mathrm{~J}\right){ }^{[64]}$ This means that we would need a surface comparable to that of France, or less than the a tenth of the Sahara desert, to supply the entire world energy demand with $17 \%$ efficiency devices. The latter is illustrated in the photovoltaic power potential world map of Figure 1.1a. The area of France, i.e. entire world energy demand, has been reproduced in several desertic regions where more than $5 \mathrm{kWh}$ can be harvested as long-term daily averages.

Fortunately, photovoltaic systems (PVSs) capable of converting around 20\% of solar energy into electric energy are already in the market. The PVSs are in general very environmental

friendly and advantageous, ${ }^{[65-66]}$ which in the present scenario make optimistic projections: the up-to-date capacity of $\sim 40 \mathrm{GW}$ is believed to be almost duplicated, accounting for nearly half of the renewables by $2040 .{ }^{[56]}$ The PVSs are mainly composed by solar panels, direct current (DC) to alternating current $(\mathrm{AC})$ inverters and several more structures depending on the application. ${ }^{[62,}$ 67] 
(a)

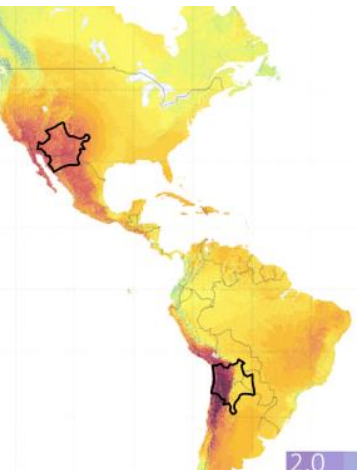

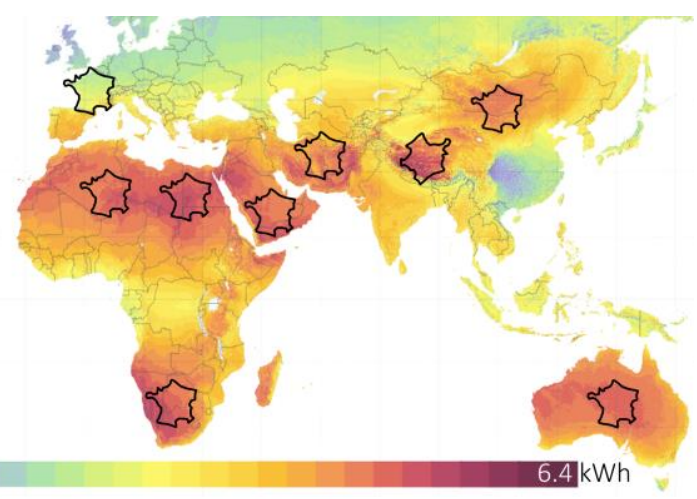

(b)

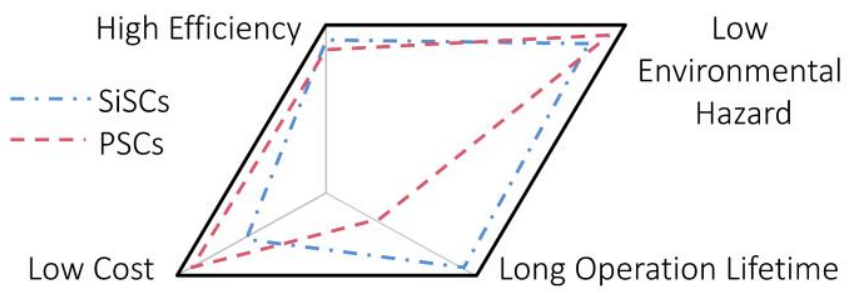

Figure 1.1. (a) Photovoltaic power potential with daily totals from The World Bank () 2019 Global Solar Atlas 2.0, Solargis. ${ }^{[68]}$ The area corresponding to France represents the world energy demand and it is reproduced in several deserts worldwide (b) Three (plus one) main aimed elements for the development of energy production technologies and the comparison (dashed lines) between current states of silicon- and perovskite-based solar cells, SiSCs and PSCs, respectively.

Extensive outdoor applications are typically classified as grid-connected, stand-alone and hybrid PVSs. First, the grid-connected PVSs are operated parallelly interconnected with the electrical grid, so they reduce the load to other energy generating technologies during the Sun hours. Second, the stand-alone PVSs are designed to be self-sufficient during the entire day, so they storage non-consumed energy during Sun hours to be consumed when dark from the storage unit (e.g. batteries). Note that the power storage unit has a significant contribution in terms of costs. ${ }^{[69]}$ The hybrid systems combine both functionalities. Nevertheless, here we focus on the solar cells which compose the solar panel, which are the core of the PV technology.

The development of every energy production technology may maximize three main aspects: high efficiencies, long operation lifetimes and low costs. In the best practices, low environmental hazard is considered a fourth main element, as in the scheme of Figure 1.1b. In terms of efficiency, the goal is to minimize the amount of energy which is dissipated. Here there are two main strategies: to optimize existing devices towards their theoretical limit and/or design new devices with higher theoretical limits. 
The perovskite solar cells (PSCs) gained the attention of most of the research community in the PVs, booming after 2013 with the establishment of the methylammonium lead iodide $\mathrm{CH}_{3} \mathrm{NH}_{3} \mathrm{PbI}_{3}$ (MAPI) as the light harvesting material. ${ }^{[49,70]}$ The MAPI is a hybrid material which belongs to the wide family known as perovskites. The perovskites have the general chemical formula $\mathrm{ABX}_{3}$ and the crystal structure of the original perovskite mineral: the calcium titanate $\left(\mathrm{CaTiO}_{3}\right)$. Such structure is illustrated in Figure 1.2a. In MAPI, the A cation is the methylammonium $\mathrm{CH}_{3} \mathrm{NH}_{3}$ (MA), the $\mathrm{B}$ cation is the lead and iodide is the $\mathrm{X}$ anion, ${ }^{[71]}$ as shown in Figure 1.2a left panel. Other total or partial substitutions typically include formamidinium $\mathrm{CH}\left(\mathrm{NH}_{2}\right)_{2}$ (FA) and/or $\mathrm{Cs}$ as A-cation and other halides like $\mathrm{Br}$ (Figure 1.2a right panel) or $\mathrm{Cl}$. Also the metallic B-cation have been substituted by $\mathrm{Sn}$ in lower bandgap absorbers. ${ }^{[72-73]}$ More than a few properties have been characterized for these materials during decades, ${ }^{[74]}$ being the ferroic nature ${ }^{[75]}$ probably the most notorious before the age of PSCs and optoelectronic applications.

The manufacturing of PSCs involves low cost materials and fabrication methods. ${ }^{[70,76-77]}$ This is the strongest pro element for PSCs in comparison with stablished technologies like silicon, ${ }^{[78]}$ as schemed in Figure 1.1b. PSCs are also very versatile, with already well performing reports for semi-transparent, ${ }^{[79]}$ flexible $^{[80]}$ and tandem ${ }^{[81]}$ applications. These elements motivated the research, allowing reproducible power conversion efficiencies (PCE) abode $20 \%$ in less than 5 years, with a latest research-cell PCE record abode $25.2 \% .{ }^{[41,82]}$ PSCs almost debuted with PCEs above $10 \%$ and their unprecedented rate of improvement (see Figure 1.2b) suggest that overcoming SiSCs is a matter of time. This makes the efficiency the second strongest favorable factor when competing with SiSCs (see Figure 1.1b). In addition, provided a proper management of the harmful elements, like $\mathrm{Pb}$, first studies estimate lower environmental impacts for industrial manufacture of PSCs, in comparison with traditional technologies. ${ }^{[83-85]}$ However, there are still several challenges to overcome in order to PSCs to reach the market, being definitely the device stability the most important one. ${ }^{[86-89]}$ This is the weakest element of PSCs in comparison with established SiSCs, as schemed in Figure 1.1b.

A bottleneck for the stabilization and general optimization of PSCs is the need for better understanding of crucial performance aspects. ${ }^{[90]}$ Here probably one of the most debated issues has been the so-called hysteresis in the current-voltage characteristic of PSCs. ${ }^{[91-96]}$ These phenomena refer to a difficulty of PSCs to deliver steady-state current at a given voltage. It is associated with slow kinetics and large low-frequency capacitances. ${ }^{[97-98]}$ Depending on previous polarization history, and other parameters like light intensity, the current and the high frequency capacitances that can be measured vary. For instance, sweeping the voltage results to produce different DC currents and capacitances at $10 \mathrm{~Hz}$ depending on the bias scan rate or direction. Moreover, transient photo-voltage and photo-current have been similarly found to evolve slowly, even in the order of minutes, and large capacitances in the order of $\mathrm{mF} \cdot \mathrm{cm}^{-2}$ are reported under illumination. ${ }^{[90]}$ These behaviors even questioned the PCE reports during the first years of development. Hence, the validation of PCE in PSCs requires special protocols for best practices. ${ }^{[99-100]}$ The reason behind these anomalous behaviors seems to be associated with slow evolving mobile ions and their interaction with electronic charge and photon fluence, in a close relation with the charge carriers recombination. ${ }^{[90]}$ 


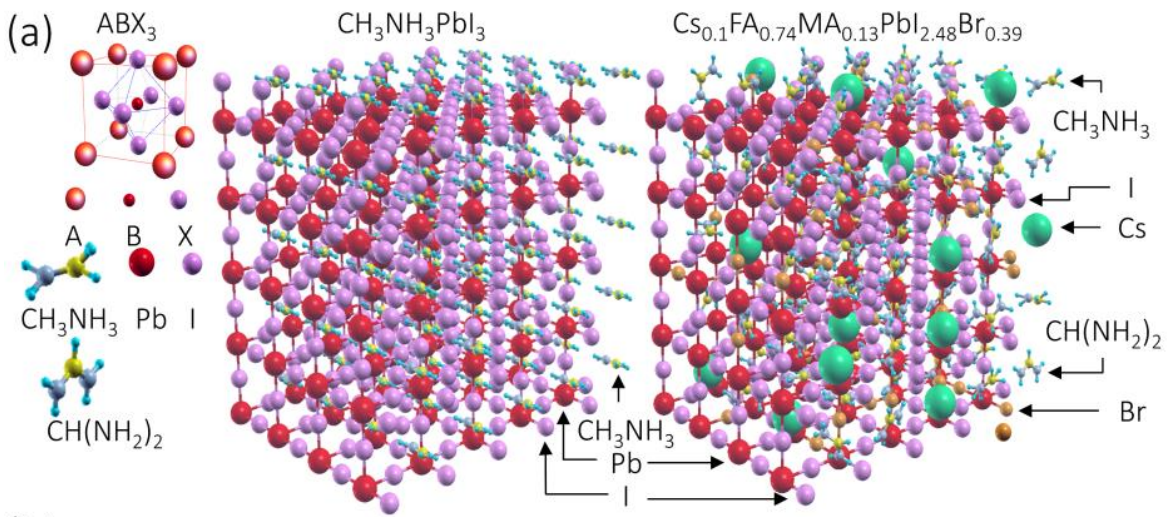

(b)

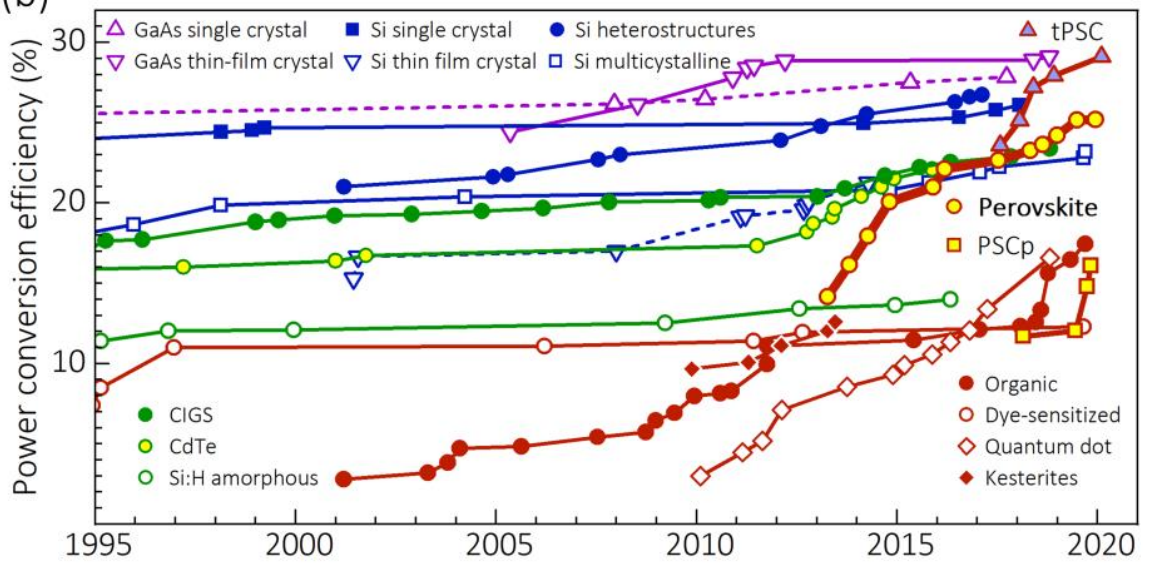

Figure 1.2. (a) Generic perovskite crystal unit cell structure, in the left, and some examples in 100 atoms arrays. In MAPI (center) $\mathrm{A}$ is the organic cation $\mathrm{CH}_{3} \mathrm{NH}_{3}, \mathrm{~B}$ is the metallic cation $\mathrm{Pb}$ and $\mathrm{X}$ is the halide anion $\mathrm{I}$. In some mixed compounds (right) a given proportion of A-cations may be substituted by $\mathrm{CH}\left(\mathrm{NH}_{2}\right)_{2}$ or $\mathrm{Cs}$ and the $\mathrm{X}$ halide can be $\mathrm{Br}$, as indicated. (b) Best research-cell efficiencies from single junction devices during the last 25 years for different technologies, including perovskite-based tandem and panel devices, tPSCs and PSCsp, respectively. Adapted from NREL. ${ }^{[41]}$

The complex kinetics of the electrical response of PSCs demands the study of several timescales and the simultaneous characterization of the mechanisms of dissipation and storage of energy, i.e. the resistive and capacitive features. In this context, the use of AC mode techniques like impedance spectroscopy (IS) can be very useful. Therefore, the combination of AC experiments and DC mode dynamic measurements of current voltage $(J-V)$ curves will be the main strategy, as discussed in following chapters. 


\subsection{Motivation and strategy}

The contribution to the sustainability of economic progress is the ultimate motivation of this work, via the development of renewable energies. Attending particularly to the case of the PVs technology, the focus here is more specifically set on the PSCs. The understanding of their physical working mechanisms will be the concrete goal along the following chapters.

Particularly, the subsequent chapters answer the questions of: what the origin of the hysteresis in the $J-V$ curves of PSCs is, and what the nature of the large capacitances and slow kinetics phenomena in these devices may be. Furthermore, this work pretends to clarify how to use and/or interpret dynamic DC $J-V$ measurements and IS analyses in PSCs. On the later, the aim is to clarify several inconsistencies with the thermal admittance spectroscopy (TAS) and the MottSchottky (MS) analyses, when they are applied to PSCs. Lastly, a new characterization technique is proposed and first tested in PSC: the light intensity modulated impedance spectroscopy (LIMIS).

The present study tackles three of the main elements in the development of the PV technology (Figure 1.1b). The understanding of the hysteresis phenomena seeks to validate the efficiency reports and, as well as the clarification of the capacitive features, contributes to the elucidation of the operation modes of PSCs, aiming their optimization. The study of the large photogenerated low-frequency capacitances is also intended to be a starting point for the possible application on super-capacitor solar cells as complementary energy storage mechanism in stand-alone and hybrid photovoltaic systems. Furthermore, for grid-connected and hybrid PVSs the large capacitance could contribute to the stabilization of the current supply to the net. The combination of energy conversion and storage in PSCs could have an extra impact in the reduction of costs. Additionally, the understanding of the role of mobile ions in perovskites and their characterization in terms of capacitance via impedance spectroscopy aims to contribute to the optimization of the device stability, increasing the lifetime of the PSCs.

In order to present the results of this work, a theoretical chapter is provided in the following. Firstly, this theoretical survey introduces the main working principles of photovoltaic solar cells and the particular case of PSCs is commented: its structure, materials and stability. Then, the general formalism of the $J-V$ curve in p-n junction based solar cells is introduces and the phenomenology of the $J-V$ curve hysteresis in PSCs is described. Subsequently, the capacitance as a general concept and in solar cells is introduced. The differences between the capacitances due to shallow levels doping density and those from deep trap levels is tackled, and the chemical capacitance mechanisms at forward biases are also introduced. Lastly, an overview on the fusion of the concepts of PSCs and supercapacitors is provided.

An experimental instrumentation chapter is included after the introductory framework. First, some context is provided on the samples which will be analyzed in subsequent chapters. I that section the labeling for each device and a general description of the fabrication process of PSCs are presented. Later, a brief description of the instrumentation details regarding dynamic DC J$V$ curve measurement precedes a deeper discussion on concept and instrumentation of IS and related methods. IS versus DC bias and temperature, MS and TAS respectively, are described. Similarly, the use of light modulating photocurrent and photovoltage, IMPS and IMVS 
respectively, are tackled. The chapter is concluded with a brief discussion on the Sawyer-Tower (ST) circuit setup and the actual ferroelectric hysteresis.

The dark characterizations and modeling are discussed in the fourth chapter. Earliest sections of the chapter deal with the material characterization of MAPI pellet samples by IS and ST methods. Then, the scan-rate and temperature dependencies of the dark $J-V$ curves are discussed. Subsequently, the dark capacitance spectra via IS are examined as a function of temperature (TAS) and DC bias (MS).

The light characterization and modeling are considered in the fifth chapter. First, the experiments of fast scan rate $J-V$ curves after pre-bias are confronted with drift diffusion simulations. Then, an IS analysis at open circuit under different illumination intensities is presented along with the corresponding equivalent circuit parametrization. In the next section, a more general analysis is made on the low-frequency capacitance at open-circuit and short-circuit under light and forward bias in dark and an explanation is proposed for the origin of these features. Lastly, the new photoimpedance technique, named LIMIS, is introduced and the preliminary results on PSCs are presented.

In a concluding chapter the main individual conclusions from each chapter are summarized. This work is focused in the understanding of the physics behind several device phenomena and the development of characterization techniques. Nevertheless, some final comments are also provided regarding the material science results displayed along the manuscript. Moreover, some comments on outlook and open research problems are also included.

After the conclusions, annexed sections present the lists of publications on which the thesis is based, as well as further publications by the author.

\section{References}

[1] F. Ferlin, Centaurus 59 (2017), 308. https://doi.org/10.1111/1600-0498.12163

[2] I. Newton, The Principia, Univ. of California Press, 1999.

[3] I. Newton, Opticks, or, a Treatise of the Reflections, Refractions, Inflections and Colours of Light (1721), General Books LLC, 2010.

[4] C. S. Roero, in Landmark Writings in Western Mathematics 1640-1940, (Ed: I. Grattan-Guinness), Elsevier Science, Amsterdam 2005, 46. https://doi.org/10.1016/B978-044450871-3/50085-1

[5] L. Debnath, Int. J. Math. Educ. Sci. Tech. 40 (2009), 353. https://doi.org/10.1080/00207390802642237

[6] B. F. J. Schonland, J. Franklin Inst. 253 (1952), 375. https://doi.org/10.1016/0016-0032(52)90717-5

[7] I. Falconer, Metrologia 41 (2004), S107. https://doi.org/10.1088/0026-1394/41/5/S01

[8] C. Cajavilca, J. Varon, G. L. Sternbach, Resuscitation 80 (2009), 159. https://doi.org/10.1016/j.resuscitation.2008.09.020

[9] R. Cecchini, G. Pelosi, IEEE Antennas Propag. Mag. 34 (1992), 30. https://doi.org/10.1109/74.134307

[10] G. W. A. Dummer, Electronic Inventions and Discoveries. Electronics from its Earliest Beginnings to the Present Day, Inst. Phys. Publ., Bristol, 1997.

[11] B. Dibner, Electr. Eng. 80 (1961), 321. https://doi.org/10.1109/EE.1961.6433241

[12] J. R. Hofmann, Osiris 3 (1987), 45. https://doi.org/10.1086/368661

[13] A. H. D. Cheng, D. T. Cheng, Eng. Anal. Bound. Elem. 29 (2005), 268. https://doi.org/10.1016/j.enganabound.2004.12.001

[14] L. Pearce Williams, Contemp. Phys. 5 (1963), 28. https://doi.org/10.1080/00107516308205836

[15] T. Jenkins, Phys. Educ. 40 (2005), 430. https://doi.org/10.1088/0031-9120/40/5/002

[16] M. S. Gupta, IEEE Trans. Educ. 23 (1980), 156. https://doi.org/10.1109/TE.1980.4321401 
[17] L. M. Fraas, in Low-Cost Solar Electric Power, Springer, Cham 2014, 1. https://doi.org/10.1007/9783-319-07530-3_1

[18] A. S. Inan, Proc. 2010 IEEE Int. Symp. Circuits Syst., Paris, (2010), p. 73. https://doi.org/10.1109/ISCAS.2010.5537049

[19] T. K. Sarkar, M. Salazar-Palma, D. L. Sengupta, IEEE Antennas Propag. Mag. 51 (2009), 97. https://doi.org/10.1109/MAP.2009.5338690

[20] H. Unz, IEEE Trans. Educ. 6 (1963), 30. https://doi.org/10.1109/TE.1963.4321796

[21] C. Susskind, IEEE Trans. Microwave Theory Tech. 36 (1988), 802. https://doi.org/10.1109/22.3599

[22] I. W. Griffiths, Rapid Commun. Mass Spectrom. 11 (1997), 2. https://doi.org/10.1002/(SICI)10970231(19970115)11:1<2::AID-RCM768>3.0.CO;2-V

[23] M. J. Klein, Arch. Hist. Exact Sci. 1 (1961), 459. https://doi.org/10.1007/BF00327765

[24] W. Wilson, Biogr. Mem. Fellows R. Soc. 5 (1960), 206. https://doi.org/10.1098/rsbm.1960.0016

[25] J. Renn, D. Hoffmann, J. Phys. B: At., Mol. Opt. Phys. 38 (2005), S437.

https://doi.org/10.1088/0953-4075/38/9/001

[26] A. Einstein, Annalen der Physik 322 (1905), 549. https://doi.org/10.1002/andp.19053220806

[27] E. Bretscher, J. D. Cockcroft, Biogr. Mem. Fellows R. Soc. 1 (1955), 68. https://doi.org/10.1098/rsbm.1955.0006

[28] F. Bloch, Zeitschrift für Physik 52 (1929), 555. https://doi.org/10.1007/BF01339455

[29] A. H. Wilson, P. A. M. Dirac, Proc. R. Soc. London, Ser. A 133 (1931), 458. https://doi.org/10.1098/rspa.1931.0162

[30] W. H. Schottky, Zeitschrift für Physik 113 (1939), 367. https://doi.org/10.1007/BF01340116

[31] W. H. Schottky, Naturwissenschaften 26 (1938), 843. https://doi.org/10.1007/BF01774216

[32] N. F. Mott, Math. Proc. Cambridge Philos. Soc. 34 (2008), 568.

https://doi.org/10.1017/S0305004100020570

[33] N. F. Mott, Proc. R. Soc. London, Ser. A 171 (1939), 27. https://doi.org/10.1098/rspa.1939.0051

[34] W. Shockley, Bell Syst. Tech. J. 28 (1949), 435. https://doi.org/10.1002/j.1538-7305.1949.tb03645.x

[35] W. Shockley, Electrons and Holes in Semiconductors with Applications to Transistor Electronics, D.

Van Nostrand, Princenton, New Jersey, 1950.

[36] W. Shockley, W. T. Read, Phys. Rev. 87 (1952), 835. https://doi.org/10.1103/PhysRev.87.835

[37] R. N. Hall, Phys. Rev. 87 (1952), 387. https://doi.org/10.1103/PhysRev.87.387

[38] D. M. Chapin, C. S. Fuller, G. L. Pearson, J. Appl. Phys. 25 (1954), 676.

https://doi.org/10.1063/1.1721711

[39] W. Shockley, H. J. Queisser, J. Appl. Phys. 32 (1961), 510. https://doi.org/10.1063/1.1736034

[40] M. A. Green et al., Prog. Photovoltaics Res. Appl. 27 (2019), 3. https://doi.org/10.1002/pip.3102

[41] NREL's Best Research-Cell Efficiency Chart, 2020, https://www.nrel.gov/pv/cell-efficiency.html.

Accessed 30.03.2020

[42] T. D. Lee, A. U. Ebong, Renewable Sustainable Energy Rev. 70 (2017), 1286.

https://doi.org/10.1016/j.rser.2016.12.028

[43] B. Gai et al., ACS Photonics 5 (2018), 2786. https://doi.org/10.1021/acsphotonics.8b00586

[44] S. P. Philipps, F. Dimroth, A. W. Bett, in McEvoy's Handbook of Photovoltaics, (Ed: S. A.

Kalogirou), Academic Press, 2018, 439. https://doi.org/10.1016/B978-0-12-809921-6.00012-4

[45] M. Freitag et al., Nat. Photon. 11 (2017), 372. https://doi.org/10.1038/nphoton.2017.60

[46] J. Zhang, L. Zhu, Z. Wei, Small Methods 1 (2017), 1700258.

https://doi.org/10.1002/smtd.201700258

[47] K. Pal, P. Singh, A. Bhaduri, K. B. Thapa, Sol. Energy Mater. Sol. Cells 196 (2019), 138.

https://doi.org/10.1016/j.solmat.2019.03.001

[48] Z. Pan et al., Chem. Soc. Rev. 47 (2018), 7659. https://doi.org/10.1039/C8CS00431E

[49] J.-P. Correa-Baena et al., Energy Environm. Sci. 10 (2017), 710.

https://doi.org/10.1039/C6EE03397K

[50] J. Urry, Theory Cult. Soc. 31 (2014), 3. https://doi.org/10.1177/0263276414536747

[51] W. Haber, Environ. Sci. Pollut. Res. Inter. 14 (2007), 359. https://doi.org/10.1065/espr2007.09.449

[52] Global Challenges for Humanity, 2014, http://107.22.164.43/millennium/challenges.html. Accessed 30.03.2020 
[53] Top Challenges for the Future of Humanity and the Planet Include Sustaining Ocean Health, Reversing Biodiversity Loss and Addressing Infectious Agents, 2015, https://en.unesco.org/news/topchallenges-future-humanity-and-planet-include-sustaining-ocean-health-reversing. Accessed 30.03.2020

[54] J. P. Holdren, Popul. Environ. 12 (1991), 231. https://doi.org/10.1007/BF01357916

[55] H. Flohn, Climatic Change 1 (1977), 5. https://doi.org/10.1007/BF00162774

[56] World Energy Outlook 2017, IEA, 2017.

[57] N. Abas, A. Kalair, N. Khan, Futures 69 (2015), 31. https://doi.org/10.1016/j.futures.2015.03.003

[58] J. M. Pearce, Futures 34 (2002), 663. https://doi.org/10.1016/S0016-3287(02)00008-3

[59] M. Höök, X. Tang, Energy Policy 52 (2013), 797. https://doi.org/10.1016/j.enpol.2012.10.046

[60] N. L. Panwar, S. C. Kaushik, S. Kothari, Renewable Sustainable Energy Rev. 15 (2011), 1513. https://doi.org/10.1016/j.rser.2010.11.037

[61] O. Ellabban, H. Abu-Rub, F. Blaabjerg, Renewable Sustainable Energy Rev. 39 (2014), 748. https://doi.org/10.1016/j.rser.2014.07.113

[62] M. A. Green, Solar Cells. Operating principles, Technology and System Applications, Prentice-Hall, 1982.

[63] V. Smil, Energy. A Beginner's Guide, One World Oxford, Oxford, 2006.

[64] L. Capuano, International Energy Outlook 2018 (IEO2018), 2018, https://www.eia.gov/pressroom/presentations/capuano_07242018.pdf. Accessed 30.03.2020

[65] A. Sendy, How the Pros and Cons of Solar Power Have Changed in 2019?, 2019,

https://www.solarreviews.com/blog/pros-and-cons-of-solar-energy\#advantages. Accessed 30.03.2020

[66] T. Tsoutsos, N. Frantzeskaki, V. Gekas, Energy Policy 33 (2005), 289.

https://doi.org/10.1016/S0301-4215(03)00241-6

[67] J. Khan, M. H. Arsalan, Renewable Sustainable Energy Rev. 55 (2016), 414.

https://doi.org/10.1016/j.rser.2015.10.135

[68] Global Solar Atlas version 2.0, 2019, https://globalsolaratlas.info/download/world. Accessed 30.03 .2020

[69] G. A. Barzegkar-Ntovom et al., Renewable Energy 152 (2020), 1302.

https://doi.org/10.1016/j.renene.2020.01.061

[70] H. J. Snaith, J. Phys. Chem. Lett. 4 (2013), 3623. https://doi.org/10.1021/jz4020162

[71] P. Gao, M. Gratzel, M. K. Nazeeruddin, Energy Environ. Sci. 7 (2014), 2448.

https://doi.org/10.1039/C4EE00942H

[72] F. Li et al., Angew. Chem. Int. Ed. 58 (2019), 6688. https://doi.org/10.1002/anie.201902418

[73] S. Shao et al., Adv. Energy Mater. 8 (2018), 1702019. https://doi.org/10.1002/aenm.201702019

[74] O. Almora, L. Vaillant-Roca, G. Garcia-Belmonte, Rev. Cubana Fis. 34 (2017), 58.

http://www.revistacubanadefisica.org/index.php/rcf/article/view/RCF_34-1_58

[75] H. Liu, X. Yang, Ferroelectrics 507 (2017), 69. https://doi.org/10.1080/00150193.2017.1283171

[76] J. Bisquert, E. J. Juárez-Pérez, P. V. Kamat, Hybrid Perovskite Solar Cells: the Genesis and Early Developments 2009-2014, Fundació Scito, Valencia, 2017.

[77] N. L. Chang et al., Prog. Photovoltaics Res. Appl. 25 (2017), 390. https://doi.org/10.1002/pip.2871

[78] Z. Song et al., Energy Environm. Sci. 10 (2017), 1297. https://doi.org/10.1039/C7EE00757D

[79] Q. Xue, R. Xia, C. J. Brabec, H.-L. Yip, Energy Environ. Sci. 11 (2018), 1688.

https://doi.org/10.1039/C8EE00154E

[80] I. K. Popoola, M. A. Gondal, T. F. Qahtan, Renewable Sustainable Energy Rev. 82 (2017), 3127. https://doi.org/10.1016/j.rser.2017.10.028

[81] T. Leijtens, K. A. Bush, R. Prasanna, M. D. McGehee, Nat. Energy 3 (2018), 828. https://doi.org/10.1038/s41560-018-0190-4

[82] M. A. Green et al., Prog. Photovoltaics 28 (2020), 3. https://doi.org/10.1002/pip.3228

[83] A. Babayigit et al., Sci. Reports 6 (2016), 18721. https://doi.org/10.1038/srep18721

[84] B. Hailegnaw et al., J. Phys. Chem. Lett. 6 (2015), 1543. https://doi.org/10.1021/acs.jpclett.5b00504

[85] T. Ibn-Mohammed et al., Renewable Sustainable Energy Rev. 80 (2017), 1321.

https://doi.org/10.1016/j.rser.2017.05.095

[86] J.-P. Correa-Baena et al., Science 358 (2017), 739. https://doi.org/10.1126/science.aam6323

[87] Y. Rong et al., Science 361 (2018), eaat8235. https://doi.org/10.1126/science.aat8235

[88] M. L. Petrus et al., Adv. Energy Mater. 7 (2017), 1700264. https://doi.org/10.1002/aenm.201700264 
[89] M. I. H. Ansari, A. Qurashi, M. K. Nazeeruddin, J. Photochem. Photobiol., C 35 (2018), 1. https://doi.org/10.1016/j.jphotochemrev.2017.11.002

[90] P. Lopez-Varo et al., Adv. Energy Mater. 8 (2018), 1702772.

https://doi.org/10.1002/aenm.201702772

[91] H. J. Snaith et al., J. Phys. Chem. Lett. 5 (2014), 1511. https://doi.org/10.1021/jz500113x

[92] E. L. Unger et al., Energy Environ. Sci. 7 (2014), 3690. https://doi.org/10.1039/C4EE02465F

[93] H.-S. Kim, N.-G. Park, J. Phys. Chem. Lett. 5 (2014), 2927. https://doi.org/10.1021/jz501392m

[94] O. Almora et al., Sol. Energy Mater. Sol. Cells 195 (2019), 291.

https://doi.org/10.1016/j.solmat.2019.03.003

[95] O. Almora et al., ACS Energy Lett. 1 (2016), 209. https://doi.org/10.1021/acsenergylett.6b00116

[96] O. Almora et al., J. Phys. Chem. Lett. 6 (2015), 1645. https://doi.org/10.1021/acs.jpclett.5b00480

[97] O. Almora, C. Aranda, G. Garcia-Belmonte, J. Phys. Chem. C 122 (2018), 13450. https://doi.org/10.1021/acs.jpcc.7b11703

[98] O. Almora et al., Nano Energy 48 (2018), 63. https://doi.org/10.1016/j.nanoen.2018.03.042

[99] J. A. Christians, J. S. Manser, P. V. Kamat, J. Phys. Chem. Lett. 6 (2015), 852.

https://doi.org/10.1021/acs.jpclett.5b00289

[100] E. Zimmermann et al., APL Mater. 4 (2016), 091901. https://doi.org/10.1063/1.4960759 


\section{Chapter 2. The physics of solar cells, perovskites and capacitance}

In the introductory chapter, the photovoltaic cells were only considered like "magic" artifacts which convert light energy into electricity. On the contrary, here a more detailed description of its working principles is depicted, with specific interest on the structure of perovskite solar cells (PSCs). Independently, a revision to the literature ${ }^{[1-5]}$ on solar cells is recommended for a better reading. This will be complemented with the phenomenological description of the current-voltage $(J-V)$ curve and formalisms of capacitance in solar cells.

\subsection{Working principles of solar cells}

The general idea of a photovoltaic cell includes two main and closely linked elements: (i) an effective absorber and (ii) a structure of selective contacts, also known as rectifying junction. These two components may guarantee three main processes: (1) photon absorption, (2) charge carrier generation and (3) charge carrier separation for selective extraction. Figure 2.1 illustrates these elements and processes.

Semiconductors are the most common materials in the photovoltaic applications, despite some mixed solid-liquid phases devices can work as solar cells ${ }^{[6]}$ following the above generalizations. Accordingly, despite the main concepts will be introduced, an understanding of solid-state physics ${ }^{[7-8]}$ and semiconductor-device physics ${ }^{[9-10]}$ is recommended for a better reading of the following contents.

The (i) absorber semiconductor material is responsible for collecting the fraction of the incident power $P_{\text {in }}$ which remains after the optical losses $P_{l o s t, o p}$ by reflection and transmission. The absorption may occur if the photon energy $E_{p h}$ is larger than the band-gap $E_{g}$ of the absorber material, i.e. the difference between the bottom and top of the conduction and valence bands, $E_{C}$ and $E_{V}$ respectively, or LUMO and HOMO in organics. Note that $E_{p h}=h v=h c / \lambda$ where $v$ and $\lambda$ are the frequency and wavelength of the photon and $c$ is the speed of light in vacuum and $h$ the Plank's constant. In the subsequent analyses the band-gap-levels-mediated absorption ${ }^{[1]}$ is neglected.

At (1) absorption, the photons transfer their energy to electrons in the top of the valence band (VB) making them go to the conduction band (CB), as in the left of Figure 2.1. This leaves an empty state in the VB with effective positive charge $+q$ : a hole. This is taken as (2) generated charge carriers.

But an absorber by itself is just a piece of material where, after (1) absorption, the (2) generated electrons $\Delta n$ and holes $\Delta p$ just (4-7) recombine. Recombination occurs after lifetimes, $\tau_{n}$ and $\tau_{p}$ at rates $U_{n}=\Delta n / \tau_{n}$ and $U_{p}=\Delta p / \tau_{p}$, for electrons and holes respectively.

The process that completes the harvesting of power $P_{\text {out }}$ is due to (ii) the diode-like structure of selective contacts. The (3) extraction of carriers may occur in a well-defined direction: the electrons are blocked towards the hole transport material (HTM) but they can scape in the 
direction of the electron transport material (ETM), while the holes cannot access the ETM and scape to the HTM. In the middle of Figure 2.1, this is the exact situation of a diode, only that in diodes the charge is injected by the current source instead that by the photon-generation, and the current flows in the opposite direction of photocurrent, typically by recombination.

(i)

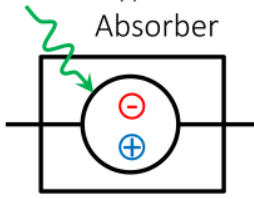

Absorber

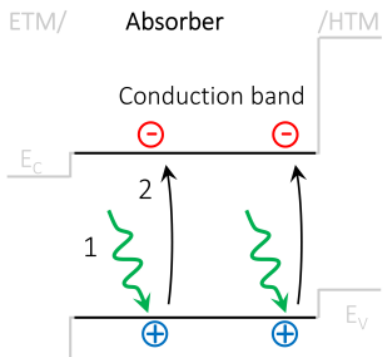

Valence band (ii)
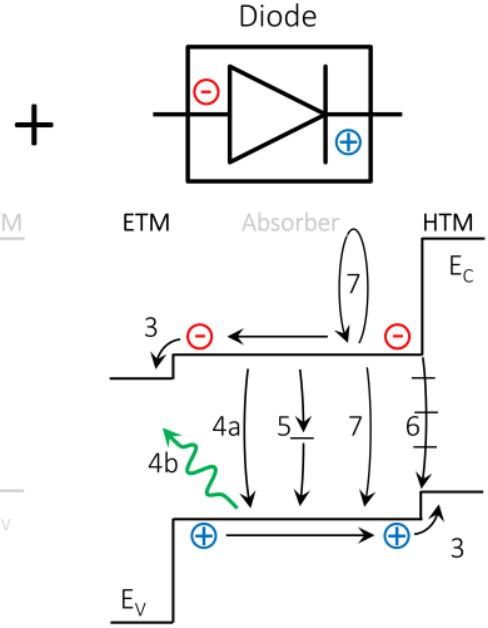
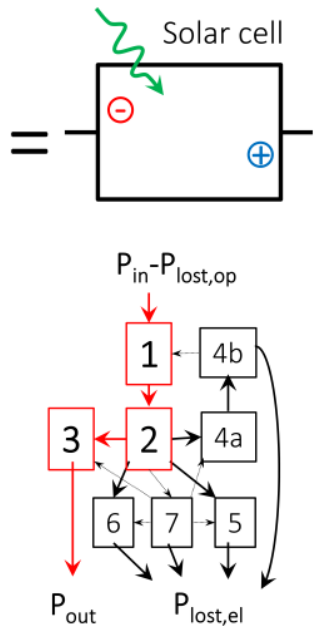

Figure 2.1. Schematics of the elements and processes in a photovoltaic cell. Above: absorber + a diode-like selective contacts structure $=$ solar cell. Wavy arrows stand for photons and circles with "-" and "+" symbol electrons and holes, respectively. Below: Energy diagram with sequence of processes occurring first at the absorber and later towards the electrodes. Total working of the cell is explained in the right bottom diagram given an incident power $P_{\text {in }}$ : (1) photon absorption; (2) electron-hole charge carriers generation; then (3) charge separation and extraction toward the respective contact to deliver a power $P_{\text {out }}$. Simultaneously, some power $P_{\text {lost }}$ is lost optically due to photon reflection and transmission $\left(P_{\text {lost }, o p}\right)$ or electrically $\left(P_{\text {lost }, e l}\right)$ when charge carriers recombine (4) radiatively or (5-7) non radiatively. Radiative recombination (4a) re-emits a photon (4b) with low probability of (1) re-absorption. Non-radiative recombination could be via (5) Shockley-Read-Hall (SRH) close mid-gap trap states, (6) surface recombination at the interfaces or grain boundaries, or the less likely (7) three particles Auger recombination, schematized just for 2 electrons and a hole case. After (7) the third carrier could recombine (4-6) or (3) being extracted. Note that $P_{\text {in }}=P_{\text {out }}+P_{\text {lost }}=P_{\text {out }}+P_{\text {lost }, \text { op }}+P_{\text {lost }, \text { el }}$. Not scaling nor band bending was considered.

The ETM and HTM are not necessarily different from the absorber. They could be sections of the same material where the conductivity is tuned. In notation terms, for thin film devices it is often used ESL and HSL as electrons and holes selective layers, respectively. The final structure of a solar cell includes metallic cathode/ETM/absorber/HTM/metallic anode. 
After charge carriers generation, not all the electrons and holes reach (3) charge extraction. Instead, they can recombine through several mechanisms: (4) radiative or (5-7) non-radiative. The dissipation of energy by the electrons in both cases makes power loses $P_{l o s t, e l}$ via photons or to the lattice, e.g. as acoustic phonons.

In the band-to-band radiative recombination (4a) the electrons in the $\mathrm{CB}$ along the bulk return to empty states in the VB and (4b) photons are re-emitted being hardly (1) re-absorbed in photonrecycling events. ${ }^{[11-12]}$ Note that here we keep the attention to the simpler direct band-gap semiconductors.

Non-radiative recombination can occur via (5) nearly mid-band-gap trap states also along the bulk. This is described by the so-called Shockley-Read-Hall (SRH) recombination model ${ }^{[13-14]}$ where the $\mathrm{CB}$ electron steps in the trap level before reaching the VB, dissipating the energy to the lattice. On the other hand, at the grain boundaries and, more importantly, at the interfaces, (6) surface recombination dominates where several trap states can mediate the path of the electron from the $\mathrm{CV}$ to the VB.

Last and least likely, after (2) carrier generation (7) the electron energy in the CB could be transferred to another electron also in the $\mathrm{CB}$ which may increase its kinetic energy before dissipating it and returning to the bottom of the CB. This is a three particle event (hole +2 electrons, or electron +2 holes -not schemed in Figure 2.1), known as Auger recombination, which allows the third particle to still contribute to (3) charge extraction or (4-6) other recombination, but wastes (1) the absorbed photon energy.

Main elements (i-ii) and processes (1-7) in the working principles of solar cells are summarized in Figure 2.1. There, the idea of power losses $P_{\text {lost }}=P_{\text {lost }, \text { op }}+P_{\text {lost }, e l}$ is also introduced, which leads to the concept of power conversion efficiency:

$$
P C E=\frac{P_{\text {in }}-P_{\text {lost }}}{P_{\text {in }}}=\frac{P_{\text {out }}}{P_{\text {in }}}
$$

which is basically the ratio between the incident power $P_{\text {in }}$ and that which can be used $P_{\text {out }}$. PCE is a common parameter for every energy conversion machine. In the case of single junction solar cells, as described in Figure 2.1, it is theoretically constrained by the Shockley-Queisser detailed balance limit. ${ }^{[15-16]}$

The natural way of estimating the PCE in solar cells is obviously the measurement of the current density $J_{\text {out }}$ and the voltage $V_{\text {out }}$ which can be used: $P_{\text {out }}=J_{\text {out }} \cdot V_{\text {out }}$. The collection of power depends on the condition of the absorber to possess the "right" $E_{g}$ in order to absorb as much as possible the given target photon flux spectrum $\Gamma$. Note that the absorber can be likewise ETM, HTM, intrinsic or a combination/sequence of these.

A conductivity type most be granted in order to be a selective material. In Figure 2.2a, the generic energy diagram of a semiconductor in several conductivity states is presented before joining them. Similarly to Figure 2.1, the levels of $E_{C}, E_{V}$, and the $E_{g}$ have been drawn. The intrinsic energy level lies approximately in the middle between the $\mathrm{CB}$ and the VB, at exactly:

$$
E_{i}=\frac{E_{C}+E_{V}}{2}+\frac{k_{B} T}{2} \ln \left[\frac{N_{V}}{N_{C}}\right]
$$


where $k_{B}$ is the Boltzmann constant, $T$ the temperature, and $N_{C}$ and $N_{V}$ are the effective densities of states in the conduction and valence bands, respectively, resulting:

$$
N_{C}=2 M_{C}\left(\frac{2 \pi m_{e}^{*} k_{B} T}{h^{2}}\right)^{3 / 2} \quad \text { and } \quad N_{V}=2 M_{V}\left(\frac{2 \pi m_{h}^{*} k_{B} T}{h^{2}}\right)^{3 / 2}
$$

Here $m_{e}^{*}$ and $m_{h}^{*}$ are the density-of-states effective masses of electrons at $E_{C}$ and holes at the $E_{V}$, respectively. Also $M_{C}$ and $M_{V}$ are the number of equivalent minima in the CB and maxima in the VB, respectively. Subsequently, de intrinsic carrier density can be obtained as:

$$
n_{i}=\sqrt{N_{C} N_{V}} \exp \left[-\frac{E_{g}}{2 k_{B} T}\right]
$$

Note that $E_{C}, E_{V}, E_{g}, E_{i}, N_{C}, N_{V}$, and $n_{i}$ characterize the semiconductor and are in principle independent on the conductivity type. Similarly the electron and hole affinities, $\chi_{n}$ and $\chi_{p}$ respectively, are introduced and defined as the energy needed for an electron in the CB or the VB, respectively, to reach the vacuum level $E_{v a c}$. The $E_{v a c}$ refers to that energy of a free stationary electron just outside the material.

The Fermi level $E_{F}$ is also depicted in Figure 2.2. The $E_{F}$ is the energy at which the probability of electron occupation of states is exactly $50 \%$ assuming the Fermi-Dirac distribution function. Moreover, the amount of energy needed by an electron to escape the material, i.e. to reach $E_{v a c}$, is known as the work function $\Phi$.
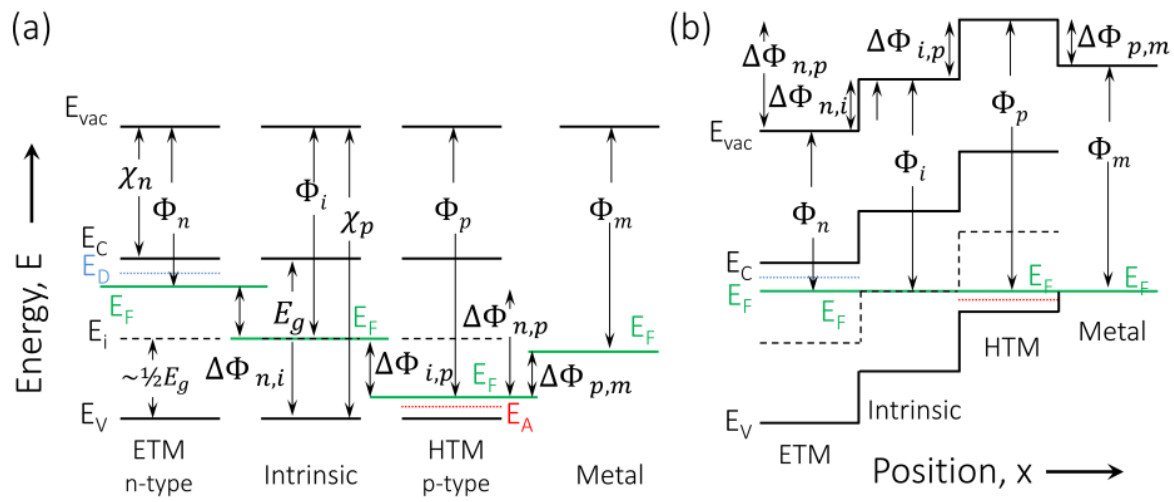

Figure 2.2. Schematics of the energy diagrams in the formation of a rectifying junction (a) before and (b) after joining the materials illustrating the aligning of the Fermi level. Not scaling nor band bending is considered.

The presence or size of the $E_{g}$ informs if it is a metal $\left(E_{g} \cong 0\right)$, a semiconductor $E_{g} \sim 1-$ $4 \mathrm{eV}$, or an insulator $\left(E_{g}>4 \mathrm{eV}\right)$. In metals, $\Phi_{m}=\chi_{n}=\chi_{p}$ is the ionization energy. Later, given a semiconductor, the position of $E_{F}$ determines intrinsic, n- or p-type character. In intrinsic semiconductors $E_{F}$ lies nearly there with $E_{i}$ in the middle of the bandgap, due to the absence or symmetry of defect levels. On the other hand, selective conductivities require shallow defect levels, typically known as doping levels in traditional semiconductors. ETM (HTM) are expected to behave like n-type (p-type) semiconductors where the shallow level of donor (acceptor) defects 
$E_{D}\left(E_{A}\right)$ makes $E_{F}$ approaching the CB (VB). Accordingly, different work functions (e.g. $\Phi_{i}, \Phi_{n}$, and $\Phi_{p}$ ) can occur with the same material

When joining the materials with different $\Phi$, the equilibrium is restored by matching $E_{F}$ at each interface. In the example of Figure $2.2 \mathrm{~b}$ a ladder-like energy diagram is evident, very similar to Figure 2.1. Distinctly, the Figure 2.2b points out how the work functions differences $\left(\Delta \Phi_{n, i}\right.$, $\Delta \Phi_{i, p}, \Delta \Phi_{n, p}, \Phi_{p, m}$ ) are mirrored by $\mathrm{E}_{v a c}$ (and also $\mathrm{E}_{i}$ ). Electrostatically, this has important consequences since the effective position $x$ dependent vacuum level gives shape to the electrostatic potential, e.g. in a one-dimensional situation:

$$
-q \varphi(x)=E_{v a c}(x)=E_{i}(x)+\left(\chi_{n}+\frac{E_{g}}{2}+\frac{k_{B} T}{2} \ln \left[\frac{N_{C}}{N_{V}}\right]\right)
$$

Therefore, the total step in $E_{v a c}$ between electrodes may result in a built-in potential $V_{b i}$, as discussed in following sections. From this we conclude that the formation of rectifying junctions requires a sequence of materials, or materials structures, with the appropriate difference in work functions.

Formation of a contact selectivity structure and effective absorption to create charge carriers are the main ideas behind the concept of photovoltaic solar cells.

\subsection{Perovskite solar cells}

As a brief introduction to PSCs, ${ }^{[17]}$ here the basic structures and materials are presented. In addition, the issue of stability is commented in a subsequent sub-section. Degradation of PSCs is not only the major problem for reaching industrial scale, it is also a matter of reliability for the characterization processes.

\subsubsection{Structure}

Detailed reviews on the material science and interface engineering can be found in the literature. ${ }^{[18-21]}$ The PSCs are typically structured as a sequence of thin film and/or nanostructured layers. The perovskite is the light harvesting material, which is comprised by the electron and hole selective layers, ESL and HSL respectively.

Despite several variations have been reported, ${ }^{[22-25]}$ here we focus on the "regular" arrangement of Figure 2.3a. There we find the $\mathrm{TiO}_{2}(\Phi \approx 3.7-4.2 \mathrm{eV})^{[26-27]}$ as ETM, which is deposited over the fluorine-doped tin oxide (FTO)/glass substrate, then the MAPI perovskite and later the spiro-OMeTAD $(\Phi \approx 3.9-5.2 \mathrm{eV})^{[28-29]}$ as HTM. The metallic contacts are often made of gold $(\Phi \approx 5.0 \mathrm{eV}) \cdot{ }^{\left[{ }^{[0-32]}\right.}$ Thus, in the regular structure the light goes first through glass, then the transparent conducting oxide (TCO), and subsequently through the $\mathrm{TiO}_{2} / \mathrm{MAPI} /$ spiro device. The regular structure is also often termed "n-i-p", despite there is no consensus on the conductivity nature of MAPI.

Also of our interest here it is the "inverted" or "p-i-n" architecture, as presented in Figure 2.3b. In this case, for the same direction of the light, the order of layers now includes indium tin oxide (ITO) as TCO, PEDOT ( $\Phi \approx 4.9-5.3 \mathrm{eV})^{[33-34]}$ as HTM, the absorber perovskite, PCBM $(\Phi \approx 4.4-5.0 \mathrm{eV})^{[35-36]}$ as ETM and quite often silver $(\Phi \approx 4.4 \mathrm{eV})^{[30,32]}$ for the metallic electrodes. 
(a)

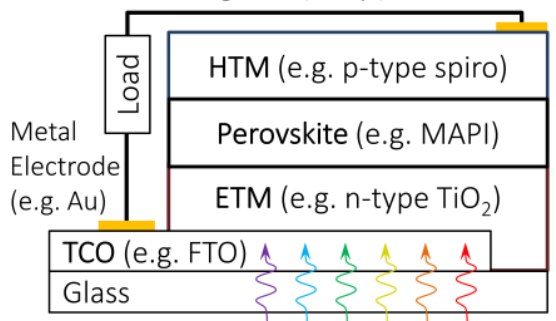

(b) Inverted (p-i-n)

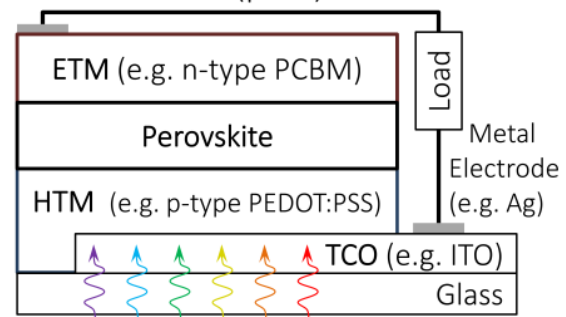

Figure 2.3. Schematics of the most common and simpler layered structures of perovskite solar cells with (a) regular and (b) inverted architectures.

\subsubsection{Materials}

The role of ETM in the regular structure is usually played by the $\mathrm{TiO}_{2}$, as in Figure 2.3a. This material is transparent to visible light $\left(\mathrm{E}_{g} \approx 3.2 \mathrm{eV}\right),{ }^{[37]}$ it has low absorption and high refractive index. ${ }^{[38]}$ The three crystalline polymorphs of $\mathrm{TiO}_{2}$ are: rutile (tetragonal), anatase (tetragonal), and brookite (orthorhombic). We are interested in the anatase $\mathrm{TiO}_{2}$, which is an indirect bandgap semiconductor with n-type conductivity due to oxygen vacancies and/or titanium interstitials. ${ }^{[37}$, ${ }^{39]}$ For the donor carrier density an order of $10^{18} \mathrm{~cm}^{-3}$ has been found. ${ }^{[40-41]}$ The distribution of the $\mathrm{TiO}_{2}$ donors levels seem to follow an exponential density of states beneath the conduction band. ${ }^{[42-43]}$

The layers of $\mathrm{TiO}_{2}$ for application in photovoltaic devices can be deposited by solution-based low cost techniques like spin coating and spray pyrolysis. ${ }^{[37]}$ As result, crystalline thin films as well as nanostructured coverings (e.g. nanotubes, nanosheets, nanorods and nanofibers) ${ }^{[44-45]}$ can be obtained on top of the FTO. In the regular structure, the $\mathrm{TiO}_{2}$ selective contact is can be found as a compact layer (flat) or as a mesoporous scaffold (meso) deposited on top of the planar compact film. Purposely, the use of a variety of nanostructures for PSCs has been reviewed in the literature. ${ }^{[46]}$

The typical ETM for the inverted structure is the fullerene derivative PCBM $\left(E_{g} \approx 2.1 \mathrm{eV}\right){ }^{[47]}$ which have been extensively used in $\mathrm{OrgPVs}^{[48]}$ in two variants: the [6,6]-phenyl-C-61-butyric acid methyl ester $\left(\mathrm{PC}_{60} \mathrm{BM}\right)$ and the [6,6]-phenyl-C-71-butyric acid methyl ester $\left(\mathrm{PC}_{70} \mathrm{BM}\right)$. For these materials, there is evidence of relatively low electron mobility in large size molecules, which influences the charge carrier transport and phase separation. ${ }^{[49]}$ Further reviews on recent progress on ETM in PSCs can be found in the literature. ${ }^{[50-51]}$

The common HTM for the regular PSCs is the 2,2'(7,7')-tetrakis-(N,N-di-p-methoxyphenylamine) $9,9^{\prime}$-spirobifluorene, i.e. the spiro-OMeTAD. This organic semiconductor $\left(E_{g} \approx 3.0\right.$ $\mathrm{eV}^{[52]}$ has also been widely characterized due to its application as HSL in solid-state dye sensitized solar cells. ${ }^{[53]}$ The spiro-OMeTAD possess a p-type self-doping which emerges during exposure to oxygen and light, besides other several extrinsic dopants which have been used to controllably oxidize the material. ${ }^{[52,54]}$ 
For the inverted structured devices, the poly(3,4-ethylenedioxythiophene) (PEDOT) doped with poly(4-styrenesulfonate) (PSS) is a typical HTM. The PEDOT:PSS $\left(E_{g} \approx 2.0 \mathrm{eV}\right)^{[55]}$ is a conjugated polymer widely used in organic photovoltaics and light-emitting devices. ${ }^{[56]}$ Several authors review on recent progress on HTM in PSCs, including also numerous buffer interlayers, e.g. fullerenes, metal oxides $2 \mathrm{D}$ capping layers. ${ }^{[57-58]}$

Besides MAPI, the family of photovoltaic absorber perovskites is very extensive. The change in compositions between the A and B cations and X anion not only modifies electrical and chemical properties aiming appropriate performance. A wide range of bandgaps has been already proved to work as absorber in PSCs, which result in performances summarized in Figure 2.4. Most of the efforts, and the best results, are on the devices with perovskite absorber $E_{g}$ within 1.1-1.7 eV, where the efficiency limit is more optimal. However, more recently, perovskite absorbers with much higher $E_{g}$ are gaining attention for semitransparent PVs in building integration, wearable applications and tandem devices. Other applications of halide perovskites include light-emitting diodes and lasers, ${ }^{[59-60]}$ and several concepts have been proposed for energy storage, like solar fuels, perovskite batteries and perovskite in supercapacitors. ${ }^{[61]}$

(a)

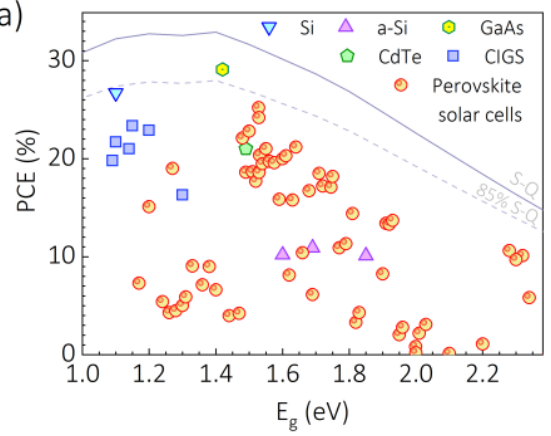

(b)

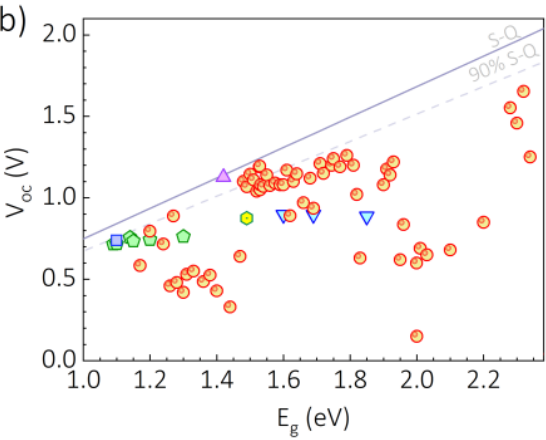

Figure 2.4. Power conversion efficiency (a) and open-circuit voltage (b) as a function of the absorber bandgap energy from PSCs and some other stablished technologies, as indicated. Experimental data (dots) are from ref. ${ }^{[62]}$ and the Shockley-Queisser (S-Q) theoretical limit data (lines) are from tabulated calculations by Rühle. ${ }^{[16]}$ Dashed lines correspond to $85 \%$ and $90 \%$ of S-Q limit in (a) and (b), respectivelly.

The MAPI absorber has been found a direct band-gap semi-conductor $\left(E_{g} \approx 1.6 \mathrm{eV}\right)^{[63-65]}$ with high absorption coefficient ${ }^{[66-67]}$ and large carrier mobility. ${ }^{[67-68]}$ One can deposit MAPI films for PV application solution-based fabrication processes, like dip and spin coating. ${ }^{[66,69]}$ The resulting layers typically show good crystalline quality at relatively high reaction rates and low temperatures. From first-principles calculations of effective masses of MAPI at room temperature, ${ }^{[70]}$ equations (2.3) and (2.4) can be evaluated giving $N_{C} \approx 5.8 \times 10^{19} \mathrm{~cm}^{-3}, N_{V} \approx$ $1.1 \times 10^{19} \mathrm{~cm}^{-3}$, and $n_{i} \approx 9.1 \times 10^{5} \mathrm{~cm}^{-3}$.

The electrical intrinsic conductivity of MAPI have been suggested to hold tuning, or "unintentional" doping. Several studies suggest that it is possible to control the concentration of donor or acceptor shallow defects, making the perovskite layer more $n$ - or p-type, respectively. ${ }^{[71-}$ 
${ }^{72]}$ However, Shi et al. ${ }^{[73]}$ suggested that p-type MAPI can be obtained by introducing impurities from groups IA, IB, or VIA, such as $\mathrm{Na}, \mathrm{K}, \mathrm{Rb}, \mathrm{Cu}$, as well as incorporating oxygen under I-rich growth conditions. On the other hand, it would be more difficult to obtain n-type MAPI given the creation of neutral defects or the compensation from intrinsic point defects.

The transition energy levels of MAPI-point-defects have been calculated by several first principle simulations. ${ }^{[72,74-75]}$ These studies suggest as dominant shallow levels close to $E_{V}$ : the vacancies of methylammonium $\left(V_{M A}\right)$ and lead $\left(\mathrm{V}_{\mathrm{Pb}}\right)$, the interstitial iodine $\left(I_{i}\right)$ and the anti-site replacement of methylammonium in a lead site $(\mathrm{MAPb})$. In contrast, the shallow levels near $E_{C}$ are the iodine vacancies $\left(V_{I}\right)$, the interstitial methylammonium $\left(\mathrm{MA}_{\mathrm{i}}\right)$ and the anti-sites of lead in MA site (PbMA) and MA in iodine site (MAI). Other deep energy levels in the bandgap are the donor interstitial leads $\left(\mathrm{Pb}_{\mathrm{i}}\right)$ and leads in iodine sites $\left(\mathrm{Pb}_{\mathrm{I}}\right)$, and the acceptor iodine in methylammonium ( $\left.\mathrm{I}_{\mathrm{MA}}\right)$ and lead $\left(\mathrm{I}_{\mathrm{Pb}}\right)$ sites.

Mixed ionic-electronic conductor character has been also suggested for MAPI. ${ }^{\text {[76-77] }}$ Specifically, activation energies for vacancy-assisted migrating ions have been calculated for $V_{I}^{-}$ and $V_{M A}{ }^{+}$to be approximately $450 \mathrm{meV}$ and $550 \mathrm{meV},{ }^{[78]}$ respectively, despite some scattering in the literature. ${ }^{[79-80]}$

\subsubsection{Stability}

Dedicated reviews can be found in the literature addressing the degradation issues of MAPI and similar photovoltaic perovskites. ${ }^{\left[{ }^{81-82]}\right.}$ This is a capital subject on the optimization of PSCs and a constant issue on characterization studies.

Here, a first significant element is the MAPI degradation in presence of moisture. This process occurs through two reactions: the generation of a MAPI hydrate phase by water incorporation and the formation of $\mathrm{PbI}_{2}$ by the desorption of $\mathrm{CH}_{3} \mathrm{NH}_{3} \mathrm{I}^{\left[{ }^{[83]}\right.}$ Afterward, $\mathrm{CH}_{3} \mathrm{NH}_{3}{ }^{+}$and $\mathrm{I}^{-}$species are lost and decompositions into $\mathrm{PbCO}_{3}, \mathrm{~Pb}(\mathrm{OH})_{2}$, and $\mathrm{PbO}$ occur. ${ }^{[84]}$ These considerations suggest encapsulation or low humidity control (below 10\%) during characterization procedures. However, even without any moisture, there are further degradation mechanisms.

Thermal instability is also an issue, ${ }^{[85]}$ considering that the solar cells working conditions are around $40-80^{\circ} \mathrm{C}$. MAPI's main tetragonal crystalline phase occurs between $160-330 \mathrm{~K} .{ }^{[63,68,86]}$ This implies phase transitions around $-113^{\circ} \mathrm{C}$ from orthorhombic and, more worrying, around $57^{\circ} \mathrm{C}$ to cubic. Irreversible critical degradation have been found over $65^{\circ} \mathrm{C}$. ${ }^{[87-88]}$ More specifically, two thermal degradation pathways have been suggested: $\mathrm{CH}_{3} \mathrm{NH}_{2}+\mathrm{HI}$ (reversible), and $\mathrm{NH}_{3}+\mathrm{CH}_{3} \mathrm{I}$ (irreversible). ${ }^{[89]}$

Also the load and electrical field affect stability. ${ }^{[87,90-91]}$ A larger chemical reactivity of the external contacts with drifted iodide ions under applied bias has been reported. ${ }^{[90-91]}$ Similarly, the diffusion of iodine and MA ions towards the electrodes or metals from the contacts have been found in encapsulated devices, which speaks of the intrinsic nature of perovskite instability. ${ }^{\text {[92] }}$

In closing, PSCs are an emerging family of devices with thin film structure and ease fabrication procedures. The absorber materials, as MAPI, have very optimal optoelectronic properties for versatile applications in the PV field. The main current challenge for PSCs is the stability which is found to depend on intrinsic and extrinsic factors. 


\subsection{The current-voltage characteristic}

Having introduced earlier the working principles of solar cells, and even some remarks on PSCs, ${ }^{[17,93]}$ the next step is to tackle their electrical response. As above mentioned, evaluating the PCE in PVs requires to measure $P_{\text {out }}$, which in an electricity generation device comes from the product $J \times V$. In this section, the presented framework of concepts and equations can be complemented with the reading of semiconductor device physics literature. ${ }^{[1,3-4,9,42]}$

The voltage responds to the difference in electrostatic potential between two points. However, note that the presence of dipoles at the interfaces justifies two potential differences to be considered: the Galvani or inner potential difference $\Delta \varphi_{G}$ and the Volta or outer potential $\Delta \varphi_{V}$, the one that can be always measured. For instance, taking a solar cell at the points 0 and $d$ corresponding to the electrodes interfaces in a one-dimensional situation, at equilibrium

$$
\begin{aligned}
& \Delta \varphi_{G}=\varphi\left(d^{\leftarrow}\right)-\varphi\left(0^{\rightarrow}\right)=-V_{b i} \\
& \Delta \varphi_{V}=\varphi\left(d^{\rightarrow}\right)-\varphi\left(0^{\leftarrow}\right)=V
\end{aligned}
$$

where the sides $0^{\rightarrow}$ and $d^{\leftarrow}$ are in the ETM and HTM, respectively, and $0^{\leftarrow}$ and $d^{\rightarrow}$ are in the metals, which considers interphase-dipoles canceling $V_{b i}$, somewhere between 0 and $L$.

The current density, on the other hand, comes from two main mechanism: drift and diffusion of charge carriers. Note that the concentrations of electrons $n$ in the conduction band and holes $p$ in the valence band may have different kinetics, which makes useful to introduce them in terms of the quasi-Fermi levels for electrons and holes

$$
\begin{array}{lrl}
n=n_{i} \exp \left[\frac{E_{F n}-E_{i}}{k_{B} T}\right], & p=n_{i} \exp \left[\frac{E_{i}-E_{F p}}{k_{B} T}\right] \\
E_{F n}=E_{i}+k_{B} T \ln \left[\frac{n}{n_{i}}\right], & E_{F p}=E_{i}-k_{B} T \ln \left[\frac{p}{n_{i}}\right]
\end{array}
$$

with respect to $E_{i}$. Note that the important concept here is that the quasi-Fermi levels reflect the deviation of the carriers concentrations at a given energy level with respect to that of the level, e.g. the deviation of $n(p)$ from $N_{C}\left(N_{V}\right)$ at $E_{C}\left(E_{V}\right)$ respectively. Also importantly, Equation (2.7a) responds to the case of non-degenerate semiconductors, where the equilibrium $E_{F}$ lies at least $2 k_{B} T$ away from $E_{C}$ or $E_{C}$ within the band-gap and thus the Fermi-Dirac distribution approaches the Boltzmann statistics.

Now we consider that in equilibrium $E_{F n}=E_{F p}=E_{F}$, which are connected with $V=0$ via Equations (2.5)-(2.7). Then, when the carrier density is modified by changing $V$, somewhere extra $\Delta n=\Delta p$ splits the $E_{F n}$ and $E_{F p}$. The change in $V$ proportionally bends $E_{i}$. But at the ETM and $\operatorname{HTM}$ (e.g. at the points 0 and $d$ ) only the minorities are significantly shifting $E_{F p}$ and $E_{F n}$, which lead us to

$$
q V \cong E_{F n}-E_{F p}
$$

Furthermore, from Equations (2.7) and (2.8) we get:

$$
n p=n_{i}^{2} \exp \left[\frac{E_{F n}-E_{F p}}{k_{B} T}\right] \cong n_{i}^{2} \exp \left[\frac{q V}{k_{B} T}\right]
$$


which in equilibrium delivers the action-mass law $n p=n_{i}{ }^{2}$.

Moreover, the presence of $V$ implies a spatial change in $\varphi(x)$ which makes the electric field $\xi(x) \neq 0$ somewhere inside the device. Charge carriers under $\xi$ are forced to move producing the drift current density

$$
J_{\text {drift }}=q\left[\mu_{n} n+\mu_{p} p\right] \xi
$$

where $\mu_{n}$ and $\mu_{p}$ are the electron and hole mobilities, respectively. Note that $\mu_{n}$ and $\mu_{p}$ are significantly influent in the transport phenomena only in the section where $\xi(x) \neq 0$ and significantly higher. On the other hand, gradients in charge concentrations produce diffusion current density

$$
J_{\text {diff }}=q\left[D_{n} \nabla n-D_{p} \nabla p\right]
$$

where $D_{n}$ and $D_{p}$ are the electron and hole diffusion coefficients, res-pectively, and $\nabla=\partial / \partial x$ is here the one-dimensional gradient operator. Contrary, note here that $D_{n}$ and $D_{p}$ are significantly influent parameters everywhere $\xi(x) \cong 0$. Nevertheless, if the Einstein relation $D=\mu k_{B} T / q$ is fulfilled, one of the two parameters can be discarded in favor of the other, making easier the description.

Given that the current should be the same everywhere in the device, the total current

$$
J(x)=J_{\text {drift }}(x)+J_{\text {diff }}(x)
$$

accounts for the redistribution of the two components. However, from the forms of equations (2.10) and (2.11), and considering (2.5) and (2.7a) and the Einstein relation, the components of the total current can be re-written as electrons and holes current densities through the CB and VB, respectively

$$
\begin{aligned}
& J(x)=J_{n}(x)+J_{p}(x) \\
& J_{n}=q\left[\mu_{n} n \xi+D_{n} \nabla n\right]=\mu_{n} n \nabla E_{F n} \\
& J_{p}=q\left[\mu_{p} p \xi-D_{p} \nabla p\right]=\mu_{p} p \nabla E_{F p}
\end{aligned}
$$

A crucial observation from Equation (2.13) is that current requires a sloping in the quasiFermi levels. Furthermore, given the same slopping, the charge carrier concentrations decide which the main contribution to current is. This is a very useful tool for drawing and reading energy diagrams.

In addition, from Equation (2.13) note that in the vicinities of $0^{\rightarrow}$ and $d^{\leftarrow}$ only one current $J_{n}$ or $J_{p}$ is typically taken, meaning that $\nabla E_{F p}$ and $\nabla E_{F n}$ are zero, respectively. Thus, back to Equation (2.8) the equality is satisfied if under bias the quasi-Fermi levels of majorities match the exact equilibrium position with respect to $E_{i}$, i.e. $E_{F n}\left(0^{\rightarrow}, V\right)=E_{F n}\left(0^{\rightarrow}, 0\right)$ and $E_{F p}\left(d^{\leftarrow}, V\right)=$ $E_{F p}\left(L^{\leftarrow}, 0\right)$. Accordingly $n\left(0^{\rightarrow}, V\right) \sim n\left(0^{\rightarrow}, 0\right)$ and $p\left(d^{\leftarrow}, V\right) \sim p\left(d^{\leftarrow}, 0\right)$ at low injection so $(2.8)$ is satisfied. But since this should be also satisfied in a broader region along the ETM and the HTL, then there $\nabla E_{F n}(V) \rightarrow 0$ and $\nabla E_{F p}(V) \rightarrow 0$, with a consequent $\mu_{n} \rightarrow \infty$ and $\mu_{p} \rightarrow \infty$, respectively. The latter is the so-called infinite charge carrier mobility approximation, which hold the equality of Equation (2.8) and the assumption of perfect contact selectivity. 
Expressions (2.10)-(2.13) are the well-known drift diffusion (DD) equations in semiconductor devices. They can be solved analytically in a very few simple cases and mostly numerically. However, for example, in (2.13) we have 2 equations and 5 unknowns. This leads us to the introduction of the continuity equations

$$
\frac{\partial n}{\partial t}=G_{n}-U_{n}+\frac{1}{q} \nabla J_{n} \quad \text { and } \quad \frac{\partial p}{\partial t}=G_{p}-U_{p}-\frac{1}{q} \nabla J_{p}
$$

where $G_{n, p}$ and $U_{n, p}$ are the generation and recombination rates for electrons and holes respectively, which are typically considered the same from the assumption that electrons and holes have only paired generation/recombination $G_{n}=G_{p}=G, U_{n}=U_{p}=U$.

The continuity equations are also very straightforward in the sense of signaling the origin of current nature in a semiconductor device. For instance, expressions (2.14) can be added to obtain the total current $J(x)=J_{n}(x)+J_{p}(x)$ assuming nearly perfect contact selectivity which leaves only $J_{n}$ and $J_{p}$ at $x_{1}$ and $x_{2}$, respectively (e.g. at left and right in Figure 2.1 energy diagram), thus

$$
J=q \int_{0}^{d}\left[G-U-\frac{1}{2} \frac{\partial}{\partial t}(n+p)\right] d x
$$

In general semiconductor devices the steady-state condition $\partial n / \partial t \cong \partial p / d t \cong 0$ is the desired solution, then only generation and recombination currents occur, as from the integral in (2.15). Moreover, substituting (2.13) in (2.14)

$$
\begin{aligned}
& \frac{\partial n}{\partial t}=G-U+\mu_{n} n \nabla \xi+\mu_{n} \xi \nabla n+D_{n} \nabla^{2} n \\
& \frac{\partial p}{\partial t}=G-U-\mu_{p} p \nabla \xi-\mu_{p} \xi \nabla p+D_{p} \nabla^{2} p
\end{aligned}
$$

By using Equation (2.16) we only need a third one to define the field and then the currents can be obtained by substitution in (2.10)-(2.13). That third one is the Poisson's equation:

$$
\begin{aligned}
& \nabla \mathfrak{D}=-\nabla^{2} \varphi=q\left[p-n+N_{\text {ion }}\right] \\
& \mathfrak{D}=\varepsilon_{r} \varepsilon_{0} \xi \\
& N_{\text {ion }}=N_{D}-N_{A}+N_{t}^{+}-N_{t}^{-}
\end{aligned}
$$

where $\mathfrak{D}$ is the electric displacement, $\varepsilon_{0}$ is the vacuum permittivity, $N_{i o n}$ is the net concentration of ions, $N_{D}$ and $N_{A}$ are the fixed ionized donors and acceptors due to shallow doping levels $E_{D}$ and $E_{A}$, respectively (see Figure 2.2), and $N_{t}^{+}$and $N_{t}^{-}$belong to fixed ionized donors and acceptors deep trap levels, respectively.

From Equation (2.17a) one can obtain $n+p=2 n+\left(\nabla \mathfrak{D} / q-q N_{\text {ion }}\right)=2 p-(\nabla \mathfrak{D} / q-$ $\left.q N_{i o n}\right)$. Substituting in the time-dependent terms of Equation (2.15) one can see the different capacitive contributions to the current. The effects related with energy storage in $\xi$ produce displacement currents $\partial \nabla \mathfrak{D} / \partial t$, in relation with geometrical capacitance $C_{g}$ and depletion layer capacitance $C_{d l}$. The change of bulk net carrier concentration $\partial n / \partial t$ gives the chemical capacitance $C_{\mu}$. The term $\partial N_{i o n} / \partial t$ may be also related with $C_{d l}$. 
Lastly, the generation/recombination models and the boundary conditions may be defined. These elements should be evaluated for each bulk segment and interphase. As in the introduction of Figure 2.1, photon generation rate and recombination rates via radiative, SRH, and Augier bulk position-dependent processes are respectively:

$$
\begin{aligned}
& G=\int_{0}^{\infty} \alpha(\lambda) \Gamma\left(\lambda, x_{0}\right) \exp \left[-\alpha(\lambda)\left(x-x_{0}\right)\right] \frac{\lambda}{h c} d \lambda \\
& U_{\beta}=\beta\left(n p-n_{i}{ }^{2}\right) \cong \beta n_{i}{ }^{2}\left(\exp \left[\frac{q V}{k_{B} T}\right]-1\right) \\
& U_{\mathrm{SRH}}=\frac{n p-n_{i}{ }^{2}}{\tau_{t n}\left(p+n_{i} \exp \left[\frac{E_{t}-E_{i}}{k_{B} T}\right]\right)+\tau_{t p}\left(n+n_{i} \exp \left[\frac{E_{i}-E_{t}}{k_{B} T}\right]\right)} \approx \frac{n_{i}}{2 \tau_{t}} \exp \left[\frac{q V}{2 k_{B} T}\right] \\
& U_{A}=\left(A_{n} n+A_{p} p\right)\left(n p-n_{i}{ }^{2}\right)
\end{aligned}
$$

In $(2.18 \mathrm{a})$ the photon generation rate $G$ is shown integrating the contributions from all the photons with different energies $h c / \lambda$ from the incident net photon flux spectrum $\Gamma$ at the material interphase in position $x_{0}$. Figure 2.5a shows some $\Gamma$ distributions due to the Sun on Earth, being of particular interest the standard AM1.5G spectrum $\Gamma_{A M 1.5 G}$, whose corresponding power density $P_{A M 1.5 G}=\int_{0}^{\infty} \Gamma_{A M 1.5 G} d \lambda$ defines the standard $1 \mathrm{sun}=100 \mathrm{~mW} \cdot \mathrm{cm}^{-2}$ illumination intensity. The position-dependency of $G_{p h}$ is due to the Beer-Lambert law ${ }^{[94]}$ where $\alpha$ is the absorption coefficient. Figure 2.5b illustrates the position- and wavelength-dependency of the spectral carrier photogeneration across a $100 \mathrm{~nm}$ thick MAPI layer where full $\Gamma_{A M 1.5 G}$ fall upon $x_{0}=0$. For thin film absorbers, $G$ is typically taken as a constant, e.g. from Equation (2.15) the distribution of Figure 2.5 may report a photocurrent of $25 \mathrm{~mA} \cdot \mathrm{cm}^{-2}$, and the same would be obtained from a constant $G \approx 4.7 \times 10^{22} \mathrm{~cm}^{-3} \cdot \mathrm{s}^{-1}$.
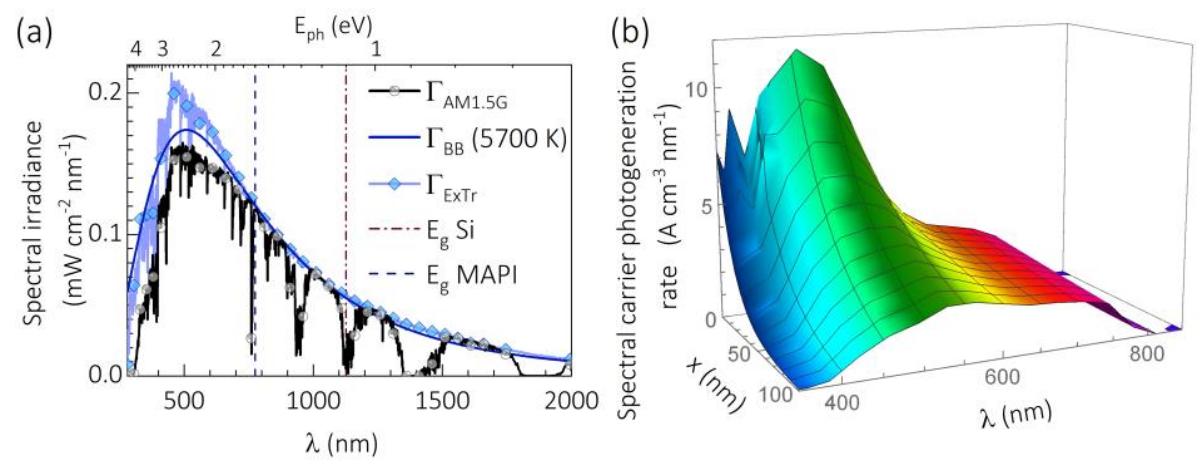

Figure 2.5. (a) Solar irradiance spectra just at the outer space of Earth $\Gamma_{E x T r}$, at Earth in the standard AM1.5 Global $\Gamma_{A M 1.5 G}$, and theoreticcal Planck's $5700 \mathrm{~K}$ black body radiation photon flux $\Gamma_{B B}$. With dashed lines, the band-gap energies of silicon and MAPI are also illustrated. (b) Spectral carrier photogeneration rate across a $100 \mathrm{~nm}$ thick MAPI layer upon incident full $\Gamma_{A M 1.5 G}$.

On the other hand, in $(2.18 \mathrm{~b}-\mathrm{d})$ first note that the negative terms in all the recombination rates actually responds to the thermal generation rate $G_{t h} \propto n_{i}{ }^{2}$. Moreover, $\beta$ is the second order band- 
to-band (most likely radiative) recombination coefficient, $\tau_{t n}$ and $\tau_{t p}$ are the SRH lifetimes for electrons and holes, respectively, and $A_{n, p}$ are the Augier recombination coefficients. $\beta$ is typically taken as the Langevin coefficient $\beta_{L}=q\left(\mu_{n}+\mu_{p}\right) / \varepsilon_{r} \varepsilon_{0}$ for low mobility semiconductors ${ }^{[5]}$ or from the detailed balance principle where for direct band-gap semiconductors $\beta \propto\left(E_{g} / n_{i}\right)^{2}$. ${ }^{[96]}$

In the case of trap mediated SRH recombination lifetimes note that they include the information on the trap carrier density with the respective capture cross section $\sigma$ and the thermal velocity $v_{t h}$ for electrons and holes: $\tau_{t n}{ }^{-1}=\sigma_{n} v_{t h, n} N_{t}, \tau_{t p}{ }^{-1}=\sigma_{p} v_{t h, p} N_{t}, \quad v_{t h, n, p}=$ $\sqrt{3 k_{B} T / m_{n, p}^{*}}$.

Within the electrodes, the boundary conditions are the continuity of displacement field (2.19a) and the constancy of total current. The former is of major importance in case of heterostructures

At the electrodes, the potential (2.19b) and the current should be set. The current boundary condition depends on the contacts type: Schottky or Ohmic. ${ }^{[97]}$ In the latter case we can take (2.19c), which for nearly perfect contact selectivity assumes recombination velocities $S_{n}(d)=$ $S_{p}(0)=0$.

$$
\begin{array}{ll}
\mathfrak{D}\left(x^{\leftarrow}\right)=\mathfrak{D}\left(x^{\rightarrow}\right) \quad \forall 0<x<d & \\
\varphi\left(0^{\rightarrow}\right)=0, \varphi\left(d^{\leftarrow}\right)=-\int_{0^{\leftarrow}}^{d^{\leftarrow}} \xi d x=V-V_{b i} & \\
J_{n}(0)=q S_{n}(0)\left(n(0)-n_{0}(0)\right) & J_{n}(d)=q S_{n}(d)\left(n(d)-n_{0}(d)\right) \\
J_{p}(0)=q S_{p}(0)\left(n(0)-n_{0}(0)\right) & J_{p}(d)=q S_{p}(d)\left(p(d)-p_{0}(d)\right)
\end{array}
$$

Note that, in this formalism, analogue extra boundary conditions (or fitting parameters) may appear at every intermediate interphase, e.g. with the discontinuities of $\xi(x)$. Furthermore, a position dependent bulk surface recombination coefficient $U_{s}$ could be considered, however its treatment would not be so different to the $U_{S R H}$ in the Equation (2.18c).

At this point we already have all the general "ingredients" for numerically solving the currentvoltage characteristic of solar cells in the classic homojunction design, or with more complicated hetero-structures. Analytically, the solution can be approximately approached in several ways as reported in the literature, ${ }^{[3-4,9]}$ e.g. by calculating the current at an advantageous position, given $J=J_{n}+J_{p}$ is the same everywhere. Also, one can integrate $G$ and $U$ along effective generation and recombination lengths as Equation (2.15). The simplest result gives:

$$
J=J_{S}\left(\exp \left[\frac{q V}{m k_{B} T}\right]-1\right)-J_{p h}
$$

where the two terms account for: the $U$-related recombination current $J_{r e c}$ and the $G$-related photocurrent $J_{p h}$. The photocurrent has none or weak dependence on $\mathrm{V}$, which create a downshifting effect on the exponential $J_{r e c}$ in our sign convention, as presented in Figure 2.6a. The absolute limit of $J_{r e c}$ towards reverse bias is the saturation current $J_{s}$, as in the inset of Figure 2.6a. 
When dark and $m=1,(2.20)$ is the celebrated Shockley equation, ${ }^{[98-99]}$ which is the ideal diode law, the reason why $m$ is called diode ideality factor. This model is obtained assuming (i) the abrupt depletion-layer, (ii) Boltzmann statistics, (iii) the low-injection, (iv) no generationrecombination current exists inside the depletion layer, and (vi) the electron and hole currents are constant throughout the depletion layer. Then

$$
J_{S}=q n_{i}^{2}\left(\frac{D_{p}}{L_{p} N_{D}}+\frac{D_{n}}{L_{n} N_{A}}\right)=q n_{i}^{2}\left(\frac{L_{p}}{\tau_{p} N_{D}}+\frac{L_{n}}{\tau_{n} N_{A}}\right)
$$

where the diffusion lengths for electrons and holes are respectively

$$
L_{n}=\sqrt{D_{n} \tau_{n}} \quad \text { and } \quad L_{p}=\sqrt{D_{p} \tau_{p}}
$$

When dark and $m \neq 1,(2.20)$ is an empirical form of the Shockley equation intended to condense into the parameter $m$ the extra exponential terms that may result from including SRH in the depletion region, or other more realistic effects. Thus $m$ is generally expected to be between 1 and 2, depending on the dominant recombination mechanism: band-to-band or mid-gap-trapmediated, respectively. Nevertheless, care must be taken, diffusion and high injection currents may also deliver ideality factors of 1 and 2, respectively.

The determination of $m$ has been tried by more than 20 different methods, ${ }^{[100]}$ most of them using (2.20) with DC forward bias. For PSCs, Tress et al. ${ }^{[101]}$ proposed the use of dark $J-V$ curves, photocurrent vs. photovoltage and electroluminescence experiments, and Almora et al. ${ }^{[102]}$ suggested IS analyses.

For the light curve two states are of central interest: the current at $V=0$, which marks the short-circuit (SC) condition and defines $J_{s c}$, and the voltage at $J=0$, which marks the opencircuit (OC) condition and defines $V_{o c}$. These parameters can be directly obtained one as a function of the other from Equation (2.20), but more importantly, they depend on the illumination intensity and the $E_{g}$ of the absorber material.
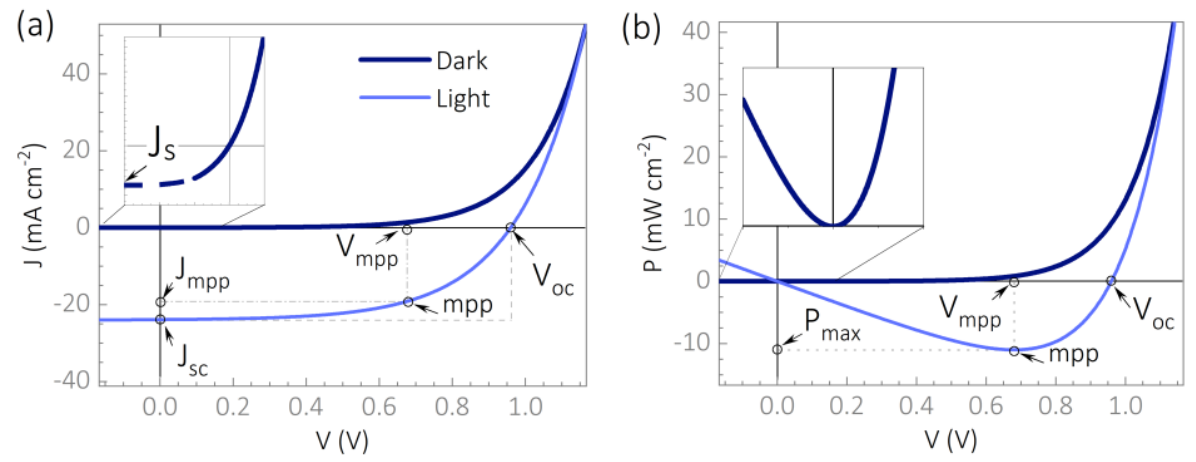

Figure 2.6. Current (a) and corresponding power (b) as a function of voltage with and without illumination of a solar cell. The insets show the magnified current/power around dark equilibrium.

From the $J-V$ behavior the corresponding power density $P=J \cdot V$ can be evaluated as in Figure 2.6b. There the dashed area corresponds to the range where $P>0$ in our convention, which represent that work made by the potentiostat (not the cell) when measuring the current. 
The work made by the cell uniquely occurs when $P<0$ in the range $0<V<V_{o c}$ (not dashed area in Figure 2.6), and such power does not need any external force to be measured: a variable load resistor is enough. Every point in the $4^{\text {th }}$ quadrant of the $J-V$ curve delivers a square $(P)$ and the largest corresponds to the maximum power point (mpp) as explicitly pointed in the light $P-V$ curve (Figure 2.6b).

At mpp the power $P_{m p p}=J_{m p p} V_{m p p}$ represents a fraction of the larger power $P_{s c-o c}=$ $J_{s c} V_{o c}$, and such ration is known as the fill factor:

$$
F F=\frac{P_{m p p}}{P_{s c-o c}}=\frac{J_{m p p} V_{m p p}}{J_{s c} V_{o c}}
$$

The $F F$ informs on the "squareness" of the $J-V$ curve, meaning that assumed an optimized/same set of $J_{s c}$ and $V_{o c}$, the higher the FF the higher the $P_{m p p}$. But making "square" the $J-V$ curve demands small and higher slopes close to SC and OC, respectively. This leads us to the concept of total differential resistance

$$
R=\left(\frac{d J}{d V}\right)^{-1}
$$

Assuming constancy of every other parameters, one can take the partial derivative and apply (2.24) to the direct current (DC) mode empirical Shockley equation (2.20), resulting the DC recombination resistance

$$
R_{\text {rec }}=R_{t h} \exp \left[-\frac{q V}{m k_{B} T}\right]
$$

where $R_{t h}=m k_{B} T / q J_{s}$ is the thermal recombination resistance.

In practice, $R_{\text {rec }}$ is limited by two main parasitic effects towards reverse bias and larger forward bias: the series and shunt resistances $R_{s}$ and $R_{s h}$, respectively. In order to explain these concepts the simplest DC equivalent circuit of a solar cell is included in Figure 2.7a, where a current source $J_{p h}$ is in parallel and opposing sign with the current through the diode: the two terms in Equation (2.20). The diode in Figure 2.7a can be also understood as a voltage dependent resistor following $R_{\text {rec }}$ as Equation (2.25).

Series resistance accounts for ohmic voltage drops $V_{\Omega}$ which reduce the voltage "felt" by the diode when the current is increased at larger forward bias. Note that here $R_{r e c} \underset{V \rightarrow \infty}{\longrightarrow} R_{S}$ instead to zero, because the current cannot exponentially increase indefinitely with $V$, surpassing the ohmic resistivity of the materials. On the contrary, at reverse bias ideally $R_{r e c} \rightarrow \infty$ but in practice instead there are leakage currents $J_{s h}$ which increase monotonically while $R_{r e c} \underset{V \rightarrow-\infty}{\longrightarrow} R_{s h}$. The inclusion of these effects in Equation (2.20) results in a transcendent equation hereon called junction operational DC current:

$$
J_{j}=J_{S}\left(\exp \left[\frac{q\left(V-V_{\Omega}\right)}{m k_{B} T}\right]-1\right)+\frac{V-V_{\Omega}}{R_{s h}}-J_{s c}
$$

where $V_{\Omega}=J_{j} R_{S} \operatorname{sgn}[P]$ (see Figure 2.6b) and $J_{s h}$ is the second term. Also Figure 2.7b,c show the $R_{s h}$ and $R_{S}$ effects on the dark $J$ - $V$ curves in each bias region.

The inclusion of parasitic resistances and the competition between different recombination mechanisms, as in Figure 2.7b,c, implies a bias-dependent ideality factor. Assuming $J_{p h}=0$ and 
a voltage range where $J \gg V / R_{S h} \gg J_{S}$ and $R_{S h} \gg(\partial J / \partial V)^{-1}$, one can obtain from the dark DC $J-V$ curve ${ }^{[102]}$

$$
m=\frac{q}{k_{B} T} J\left(\frac{\partial J}{\partial V}\right)^{-1}
$$

Similarly, considering $R_{s h}$ large enough that $J_{p h} \gg V_{o c} / R_{s h} \gg J_{s}$ and $R_{s h} \gg\left(\partial J_{s c} / \partial V_{o c}\right)^{-1}$, from the DC $J_{S C}-V_{o c}$ curve under illumination

$$
m=\frac{q}{k_{B} T} J_{s c}\left(\frac{\partial J_{s c}}{\partial V_{o c}}\right)^{-1}
$$

(a)

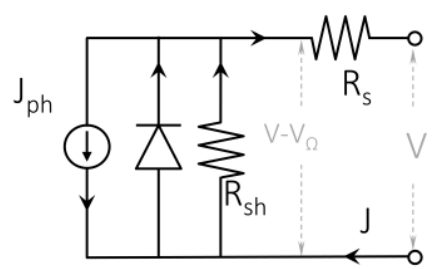

(b)

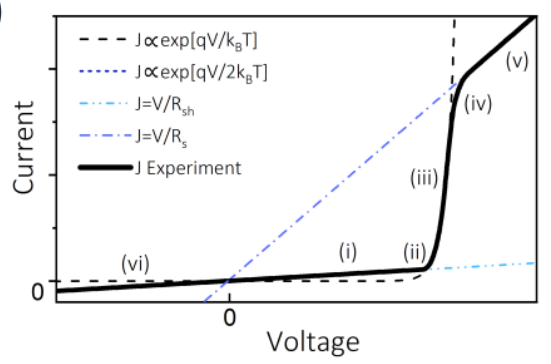

(c)

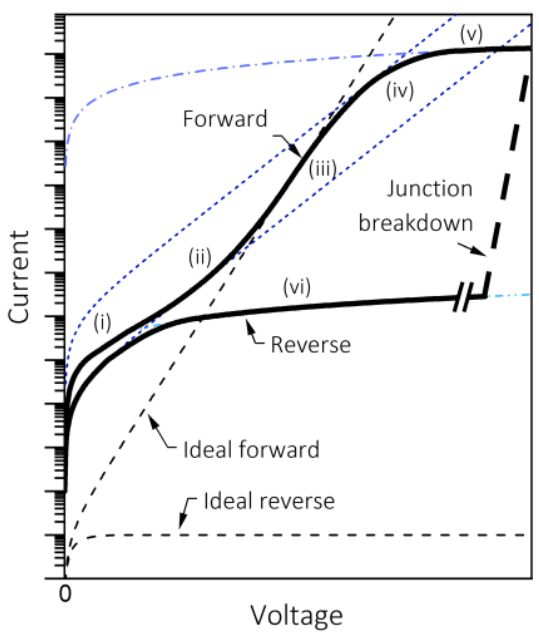

Figure 2.7. DC equivalent circuit model of a solar cell (a) and respective dark $J-V$ characteristics in linear (b) and semi-log (b) scales. (i) Ohmic-like shunt resistance effect related with leakage currents due to generation-recombination and surface effects. (ii) $J \propto$ $\exp \left[q V / 2 k_{B} T\right]$ due to SRH non-radiative recombination in the depletion region. (iii) $J \propto$ $\exp \left[q V / k_{B} T\right]$ due to diffusion in the QNR and band-to-band radiative recombination in the depletion region. (iv) $J \propto \exp \left[q V / k_{B} T\right]$ due to high injection. (v) Ohmic-like series resistance effect. (vi) Ohmic-like shunt resistance effect similar to region (i), but in reverse until junction breakdown, which transits to region (v).

Alternatively, given the bias dependent resistance, irrespective of the dark or light conditions, and measured by DC or AC method, one can define the proper integration limits so

$$
m=\frac{q}{k_{B} T} R \int \frac{d V}{R}
$$

Finally, at this point the PCE in solar cells can be addressed by taking $P_{\text {out }}=P_{m p p}$ and, for the standard, $P_{\text {in }}=P_{A M 1.5 G}$ in Equation (2.1), thus:

$$
P C E=\frac{P_{m p p}}{P_{A M 1.5 G}}=\frac{J_{m p p} V_{m p p}}{P_{A M 1.5 G}}=\frac{F F J_{S C} V_{o c}}{P_{A M 1.5 G}}
$$


In summary, PCE is the most important performance parameter which can be extracted from the $J-V$ characteristic. It is closely related with the complementary performance parameters $V_{o c}$, $J_{S C}$ and $F F$. In order to understand the $J-V$ curve and thus to propose strategies for improving PCE, the simplest model there is includes the modeling parameters $R_{s}, R_{s h}$ and $m$. Further analyses may consider the numerical solution of the transport equations.

\subsection{Phenomenology of the hysteresis in perovskite solar cells}

In practice, steady state DC $J-V$ characteristics are typically easily measured. For example, $1.0 \mathrm{~s}$ of relaxation for the measured currents at each applied voltage is usually more than enough to experimentally obtain the time-independent solution of the transport equations, i.e. $d n / d t=0$ in (2.16). This $1.0 \mathrm{~s}$ delay between sampled voltages results a scan rate of $s=10 \mathrm{mV} \mathrm{s}^{-1}$, if taking 100 points in a $1.0 \mathrm{~V}$ measurement window. This is slow, and we typically obtain almost exactly the same $J-V$ curve disregarding of the direction of the sweeping voltage and for scan rates even $10^{3}$ times faster. However, achieving steady state is not that easy for PSCs where hysteretic-like features arise, as early noticed in 2014. ${ }^{[103-105]}$

Measuring $J-V$ curves in PSCs may result in a different current patterns for the same voltage range depending of the measurement conditions. Most typically, voltage sweeping scan directions and scan rates are critically influent. Actually, these elements were the first identified and so they gave the denomination of "hysteresis" to this dynamic time evolution of the $J-V$ characteristic in PSCs. For convenience, here the voltage sweeping scan from OC $\left(V_{o c}\right)$ to $\mathrm{SC}(0 \mathrm{~V})$ is defined as the forward-to-reverse bias scan FR, and vice versa as the reverse-to forward bias scan RF. Some typical patterns of hysteresis in PSCs are presented in Figure 2.8 where the FR and RF scan directions are signaled by left- and right pointed arrows, respectively.

Importantly, in the insets of Figure 2.8 the corresponding $P-V$ curves are also displayed for each case evidencing the first major issue from the hysteresis: the misestimating of the PCE. It was early clear that in MAPI-based PSCs the FR scan delivered a higher $P_{m p p}$ than that of the RF scan, as in the cases of Figure 2.8a-c. This is hereon called normal hysteresis NHyst, while the opposite case will be referred as inverted hysteresis IHyst. The latter is usually present in mixed compounds-based PSCs and it is typified in Figure 2.8d.

Now, a qualitative description for each pattern on focus in Figure 2.8 is provided as follows. The first case in Figure 2.8a is the symmetric scan rate-dependent NHyst, where $J-V$ curves in both directions split equally collapsing to quasi-steady-state $J_{j}$ (dashed line) as the scan rate is reduced:

$$
J=J_{j}+J_{\text {cap }}
$$

where the hysteretic capacitive currents keep the sign of the scan rate and scale with the capacitance

$$
J_{\text {cap }} \approx s \cdot C
$$

Then the hysteresis would be just due to a significantly high capacitance, which charge and discharge when sweeping voltage in RF or FR directions, respectively. Importantly, in the 
simulation of Figure 2.8a a constant $C$ was used reporting a clearly symmetric change in the $J_{s c}$ and almost no change in $V_{o c}$. However typically it could be also that $C \propto \sqrt{V}$ or $C \propto$ $\exp \left[q V / m_{C} k_{B} T\right]$ (see Section 2.6). Nevertheless, what makes this behavior symmetrically distinctive is the currents collapsing to $J_{j}$ linearly with $s$ due to $C$ constant in time.

(a)

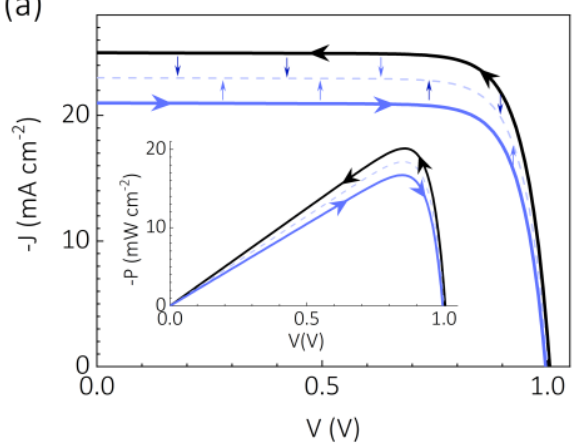

(b)

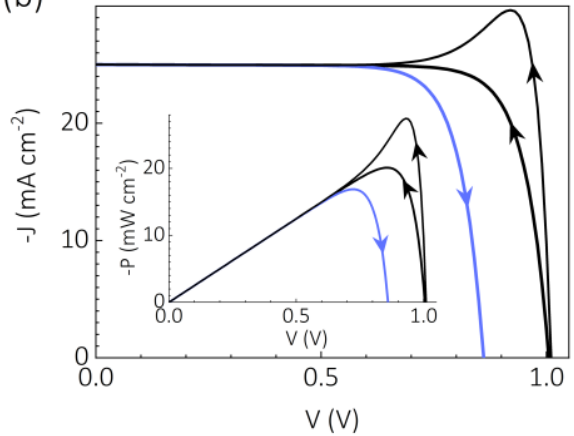

(c)

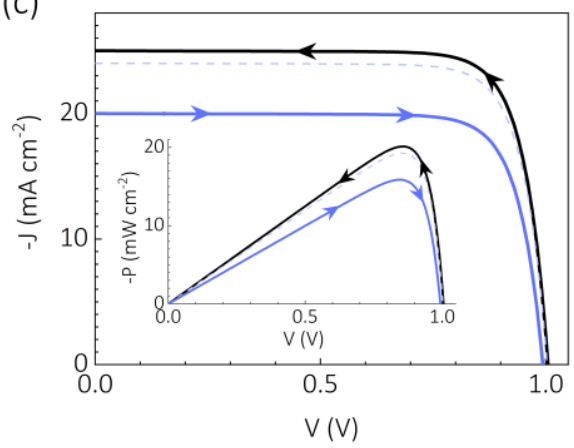

(d)

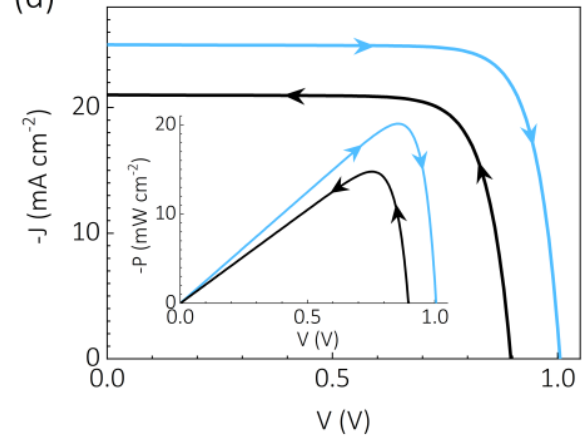

Figure 2.8. Simulation of general experimental patterns of the hysteresis in the $J-V$ curve of PSCs. Indicated with in-line arrows the $V$-sweep directions FR or RF. In the inset, the corresponding $P-V$ curves are also shown. The dashed lines stand for the $J_{j}$ and in (a) the out-line vertical arrows indicate the trend of the curves when diminishing scan rate making them collapse to $J_{j}$.

Equations (2.31) and (2.32) could also be the case of the NHyst in Figure 2.8b with $C \propto$ $\exp \left[q V / m_{C} k_{B} T\right]$ where two sub-cases may occur depending on the ratios between $m_{C}$ and the ideality factor $m$ from the $J_{j}$. First when $m_{C} / m \approx 1$ the $V_{o c}$ is reduced an amount $\Delta V_{o c}$ when RF respect to FR, with almost no change in $J_{s c}$. But if $m_{C} / m \rightarrow 2$ a kink results around the mpp, in addition to the $\Delta V_{o c}$ effect. The kink implies FFs abode $100 \%$ and thus apparent PCEs beyond theoretical predictions. This indicates the presence of measurement artifacts. In practice, the $\Delta V_{o c}$ effect and the kinks only occur in a narrow range of $s$ and sometimes only after certain prepolarization and/or pre-illumination routines. Hence there is no $s$-linear collapsing to $J_{j}$ and $C$ is significantly time-dependent. 
Similarly, the effects of polarization and illumination history can create asymmetries with respect to one of the sweeping scans, in a sort of $J_{s c}$ down shifting an amount $\Delta J_{s c}$. For instance in the case of NHyst in Figure 2.8c the equations (2.31) and (2.32) could still hold again, and even a $s$-graded collapse to $J_{j}$ can be experimentally found more often. However, in this third pattern $C$ should be also time dependent and significantly larger for RF than for FR direction.

Last but not least, in the IHyst pattern of Figure $2.8 \mathrm{~d}$ the capacitive behavior is overlapped by other mechanisms hardly explained in terms of capacitances. In this case $-\Delta V_{o c}$ and $-\Delta J_{s c}$ modifications occur in a way that now it is the RF scan the one with the higher $P_{m p p}$. In practice IHyst is not always that straightforward as in Figure 2.8d and some crosses between the RF and FR curves may occur, and even no change in $V_{o c}$ or $J_{s c}$ but in FF. This leads us to the need to generalize Equation (2.31) by including non-capacitive hysteretic currents:

$$
J=J_{j}+J_{\text {cap }}+J_{\text {noncap }}
$$

In sum, hysteretic currents can be measured in PSCs comprising variate contributions, like large displacement and faradaic currents, in addition to the electronic operational currents. Importantly, a steady state is hard to achieve since the slow nature of the capacitive currents dynamically modifies the boundary condition of the transport laws ruling the electronic currents.

\subsection{Capacitance definition and capacitive currents}

The previous section phenomenologically introduced the hysteresis in PSCs and, particularly, the role of $C$-related hysteretic currents. This particularly remarks the extra motivation in PSCs regarding the characterization of $C$. Nevertheless the hysteresis-like capacitive features are not an exclusive issue of PSCs, but a general typical effect in large surface solar cells, i.e. solar PV panels. ${ }^{[106-108]}$ Additionally, the characterization of $C$ in solar cells is a powerful tool for the study of defect densities and carrier lifetimes, as subsequently explained.

A capacitor, also known as condenser or condensator, is a two-terminal electronic component that stores electrical energy in form of electric field. Every space between two charge-holding objects or conductors at different electric potential can be considered as a capacitor. The proportion of differential change in the charge density $Q$ per differential change of voltage in a capacitor is called total differential capacitance per unit area

$$
C=\frac{d Q}{d V}
$$

In terms of the total stored energy, it can be also defined

$$
C=\frac{1}{V} \frac{d E}{d V}
$$

The two metallic terminals are often termed plates, regardless of the geometry, in analogy with the planar parallel-plate capacitor. This latter model states what is called the geometric dielectric capacitance

$$
C_{g}=\frac{\varepsilon_{0} \varepsilon}{d_{g}}
$$


where $d_{g}$ is the effective distance between plates/metallic electrodes. In this model the charge accumulates at the metallic plates (not in the dielectric nor vacuum) and for a given $d_{g}$ the amount of charge only depends on the polarizability of the media within the plates.

Expression (2.36) reflects $C_{g}$ to be constant at any $V$, then the steady-state charge at the plates comes after the integration of (2.34) as

$$
C=Q / V
$$

The integral capacitance of (2.37) is the most practical definition when $C$ is $V$-independent, like $C_{g}$.

The AC equivalent circuit of a real capacitor under measurement conditions is presented in Figure 2.9a, which includes ohmic voltage loses $V_{\Omega}$ through the series resistor $R_{S}$ and some leakage currents through a parallel resistor $R_{p}$. This is also the simplest AC equivalent circuit model which can be used to simulate a solar cell. Note that differently to the DC circuit in Figure 2.7, the AC approach does not "see" the photo generated current and the $R_{p}$ is not only accounting for the $R_{s h}$ but also for the recombination resistance from the diode.

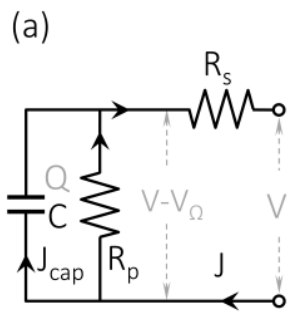

(b)

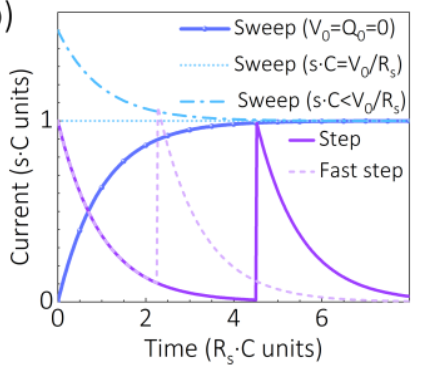

(c)

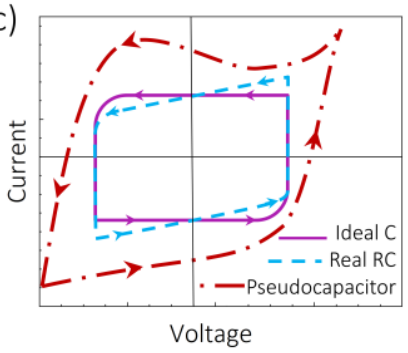

Figure 2.9. (a) AC equivalent circuit for a real capacitor under measurement and the simplest AC equivalent circuit for a solar cell. (b) Ideal displacement currents $J=J_{\text {cap }}$ when $R_{p} \rightarrow \infty$ in (a) for several cases of stepped or swept voltage, as indicated. (c) Cyclic voltammetry patterns with sweep direction as arrows for ideal, real and pseudocapacitors.

By making $R_{p} \rightarrow \infty$ in Figure 2.9a only displacement current is allowed to flow through the circuit. These capacitive currents $J_{\text {cap }}$ may depend on the polarization routine applied to the capacitor. For instance, when constant stepped or continuous swept voltage with constant scan rate $s$, basic Kirchhoff's circuits voltage law leads to

$$
\frac{\partial Q(t)}{\partial t}+\frac{Q(t)}{R_{S} C}=\frac{V_{0}+s \cdot t}{R_{s}}
$$

with the time initials $Q(0)=Q_{0}, V(0)=V_{0}$. Note that for stepped changes of voltage $s=0$ and $V(t)=V_{0}$ until the next voltage step, while in the sweeping condition the $V(t)$ is always changing. The derivative of equation (2.38) gives directly the current

$$
J_{\text {cap }}=s \cdot C\left(1+\left(\frac{V_{0}}{R_{S} s C}-\frac{Q_{0}}{R_{S} s C^{2}}-1\right) \exp \left[-\frac{t}{R_{s} C}\right]\right)
$$


There the characteristic relaxation time $\tau=R_{S} C$ determines the current evolution. This is illustrated in Figure $2.9 \mathrm{~b}$, as well as the transition to the sweep regime, when $J_{\text {cap }} \cong s \cdot C$ when the exponential term fades in (2.39), approaching (2.32). Figure 2.9c displays the situation of at least two consecutive voltage sweeps between symmetric biases in the opposite directions, also known as cyclic voltammetry. An ideal capacitor may deliver $J_{\text {cap }}$ as (2.39) in a nearly perfect square $J-V$ pattern, as the solid lines in Figure 2.9c.

Real capacitors would include leakage currents through the bulk material between the plates, like a parallel resistor. This is not considered in (2.39), where $s$ and $C$ were assumed constant despite in practice they could be bias and hence time dependent. Nevertheless, independently of the exact corrections, the general response from a real pure real capacitor in a cyclic voltammetry experiment may look like the dashed lines in Figure 2.9c. The ohmic slope of the resistance "tilts" the squared $J-V$ curve of the ideal case. Furthermore, additional non-capacitive distortions, like kinks, are characteristic of faradaic currents due to reversible redox processes. This is known as pseudocapacitance behavior. ${ }^{[109-110]}$

The charging/discharging experiments can be a straightforward and intuitive way to analyze capacitive processes and estimate time constants. This is the main idea behind some characterization techniques like transient photovoltage (TPV) ${ }^{[11-112]}$ and photo-induced charge extraction $(\mathrm{CE})^{[112-113]}$ where the carriers recombination kinetics of photosensitive devices is tested via time dependent photovoltage charging/discharging.

Alternatively, and complementary to the transient time-domain experiments, the spectroscopic frequency-domain approach is a very useful strategy. Most representative of the latter is the impedance spectroscopy (IS) characterization technique, which is the standard way to access the differential capacitance. The IS spectra also allow to elucidate resistance and conductivity modes in materials and devices, as further explained in Chapter 3.

\subsection{Capacitance in solar cells}

The simplest capacitance from any sample between two electrodes is that of the geometrical dielectric $C_{g}$, as introduced in (2.36). In the cases of thin film and other emerging PVs like organic or PSCs, a sequence of different materials compose the cell (see Figure 2.3). Therefore, $l$ layers in series rewrites (2.36) as

$$
C_{g}=\left(\sum_{l} \frac{1}{C_{g, l}}\right)^{-1}=\left(\sum_{l} \frac{d_{l}}{\varepsilon_{0} \varepsilon_{l}}\right)^{-1}
$$

where $d_{l}$ is the thickness of the $l^{\text {th }}$ layer and $\varepsilon_{l}$ the corresponding dielectric constant in the high frequency range from the impedance spectra.

However, all-solid-state solar cells are typically composed by semiconductors, whose charge carrier concentration upon perturbation is significant, differently to dielectrics. Hence, the capacitance of a solar cell fits equation (2.34) as ${ }^{[114-116]}$

$$
C=q\left|\frac{\partial}{\partial V} \int_{0}^{d} n d x\right|
$$


where the integral is taken between the electrodes at positions $0^{\rightarrow}$ and $d^{\leftarrow}$, and the bias derivative impose the condition of mobile charge carriers to the electrons and holes concentrations $n$ and $p$, respectively. The equation (2.41) can analogously be expressed in terms of holes concentration. Also note that thicknesses in (2.36) and (2.41) are the same, only that conceptually $d=d^{\leftarrow}-0^{\rightarrow}$ and $d_{g}=d^{\rightarrow}-0^{\leftarrow}$.

Expression (2.41) highlights the importance of the integration space region and the applied voltage for determining the capacitance. Accordingly, within the depletion region, and at reverse bias and low forward bias, the depletion layer capacitance $C_{d l}$ related with doping and deep levels will be discussed in Section 2.6.1 and Section 2.6.2, respectively. ${ }^{[117]}$ Later, at larger forward biases, when depletion zone contributions are negligible, the diffusion or chemical capacitance $C_{\mu}$ will be tackled in Section 2.6.3.

\subsubsection{Depletion capacitance I: Mott-Schottky analysis}

In Section 2.1 the built-in voltage $V_{b i}$ was introduced as the total step electrostatic Galvani potential, in (2.5) and (2.6). Having a $V_{b i}$ is a necessary condition for the charge selectivity in a solar cell. Following the notation of Figure 2.2,

$$
q V_{b i}=\Delta \Phi=\left|\Phi_{1}-\Phi_{2}\right|
$$

where the absolute work function step $\Delta \Phi$ is taken between selective contacts 1 and 2, i.e., $\Delta \Phi_{n, p}$ in p-n junctions or $\Delta \Phi_{n, m}$ or $\Delta \Phi_{p, m}$ in Schottky diodes. ${ }^{[9,118]}$

As introduced in Poisson's equation (2.17), the origin of the rectifying structure (2.42a) lies in the appropriate distribution of acceptor and/or donor type fixed ionized defects characterized by the concentrations $N_{A}$ and $N_{D}$, respectively. These also called doping concentrations are mainly due to shallow trap levels which shift the Fermi level towards the conduction or valence bands. Then, from energetic analysis on the position of the Fermi level, and (2.7), an approximation can be made neglecting dipoles for $\mathrm{p}-\mathrm{n}$ junctions of non-degenerate semiconductors as

$$
V_{b i} \approx \Delta \chi_{n}+\Delta E_{g}+\frac{k_{B} T}{q} \ln \left[\frac{N_{A}}{n_{i}}\right]+\frac{k_{B} T}{q} \ln \left[\frac{N_{D}}{n_{i}}\right]
$$

where the absolute differences in electron affinity $\Delta \chi_{n}$ and band gap $\Delta E_{g}$ account for heterojunctions.

However, the use of (2.42) to determine $V_{b i}, N_{A}$ and/or $N_{D}$ can be problematic due to the several experimental techniques that could be needed. Besides, as important as knowing these parameters is to determine their space distribution. An alternative approach is provided by the capacitance in dark and under reverse bias or forward DC voltages enough lower than $V_{b i}$.

Upon the formation of a p-n junction, the weakly bonded electrons in the neutral donor defects of the $\mathrm{n}$-side reach the closest holes in the neutral acceptor defects of the p-side. As result, fixed ionized space charge regions (SCRs) with widths $w_{n}$ and $w_{p}$ are formed at the n- and p-side of the junction, respectively, leaving a quasi-neutral region (QNR) elsewhere. This is illustrated in Figure 2.10a for the case of the one-sided abrupt (OSA) junction, meaning that one of the defect concentrations is much higher than the other, e.g. $N_{D} \gg N_{A}$. Since the neutrality of the device must be granted, within the space charge region 


$$
N_{D} w_{n}=N_{A} w_{p}
$$

For the OSA junction the total depletion region width $w=w_{n}+w_{p}$ is mostly only the section on the lightly doped semiconductor, e.g. $w \approx w_{p}$ in Figure 2.10a.

In equilibrium, $w$ only depends on the dielectric properties of the material(s). The corresponding energy diagram depends on the doping densities and $V_{b i}$, and one can solve the Poisson's equation (2.17) with the neutrality condition (2.43), full ionization (no majority carriers) and adding external voltage to get the DC bias dependent depletion layer width ${ }^{[9]}$ as

$$
w=\sqrt{\frac{2 \varepsilon_{0} \varepsilon}{q N}\left(V_{b i}-V\right)}
$$

where the doping concentration $N$, as well as $\varepsilon$ and the voltage dependency inside the parenthesis are effective parameters, depending on the model (see Table 2.1.). Upon AC voltage small perturbation $\tilde{V}$ there will be a consequent $\widetilde{w}$ change which implies a dynamic ionization of neutral defects at the edges of the depletion region towards the QNR; for the OSA junction $\widetilde{w}$ is mostly in the lower doped semiconductor, e.g. p-side in Figure 2.10a.

Applying definition (2.41) to the OSA junction may result in the equilibrium situation depicted in Figure 2.10b. Smaller bulk contributions from the depletion zone and QNR, are the neutral capacitance $C_{N}$ and the chemical capacitance $C_{\mu}$, respectively. ${ }^{[16]}$ In both cases the definition of capacitor plates like (2.36) makes no sense: at every position $x$ there are as many electrons as holes. Thus, contrary to dielectric $C_{g} \propto d^{-1}$, the bulk capacitance increases with thickness; e.g. $C_{N} \propto w$ and $C_{\mu} \propto(d-w)$. The influence of $C_{N}$ reverse bias can in practice be neglected, ${ }^{[119]}$ and under forward bias it would be even lower or none. On the other hand, $C_{\mu}$ is of mayor importance when $V \rightarrow V_{b i}$, as further discussed in Section 2.6.3. However, at the edges of the depletion zone an incremental voltage change $\partial V$ ionizes the same incremental charge $d Q_{d l}$ at each end of the space charge, but with opposite sign in each side. This is a typical behavior of a parallel-plate capacitor. Thus, equation (2.41) is rewritten as ${ }^{[15-116]}$

$$
C_{d l}=\frac{q}{2}\left|\frac{\partial}{\partial V} \int_{0^{\rightarrow}}^{d^{\leftarrow}}(n-p) d x\right| \quad \text { or } \quad C_{d l}=\frac{q}{2}\left|\int_{0^{\rightarrow}}^{d^{\leftarrow}} \frac{n-p}{n+p} \frac{\partial \rho}{\partial V} d x\right|
$$

which results, similarly to (2.36), in the homojunction depletion layer capacitance per unit area:

$$
C_{d l}=\frac{\varepsilon_{0} \varepsilon}{w}
$$

In the depletion approximation the voltage drop in the QNR is negligible, so $C_{d l}$ and $C_{g}$ are approximately at the same $V$, thus from $w \ll d$ then $C_{d l} \gg C_{g}$. However, as reverse bias is increased, $w$ is enhanced to the limit $d$, then $C_{d l} \rightarrow C_{g}$ and full depletion is achieved. On the contrary, if forward biases lower than $V_{b i}-V_{t h} / 2$ are applied, $w$ shrinks up to the limit of the Debye length

$$
L_{D}=\sqrt{\frac{\varepsilon_{0} \varepsilon k_{B} T}{q^{2} N}}
$$


With plate separation $L_{D}$, at the junction and/or the interfaces there will be double layer Debye capacitances

$$
C_{D}=\frac{\varepsilon_{0} \varepsilon}{L_{D}} .
$$

which are significant when $w \rightarrow L_{D}$, due to the series connection between $C_{d l}$ and $C_{D}$.
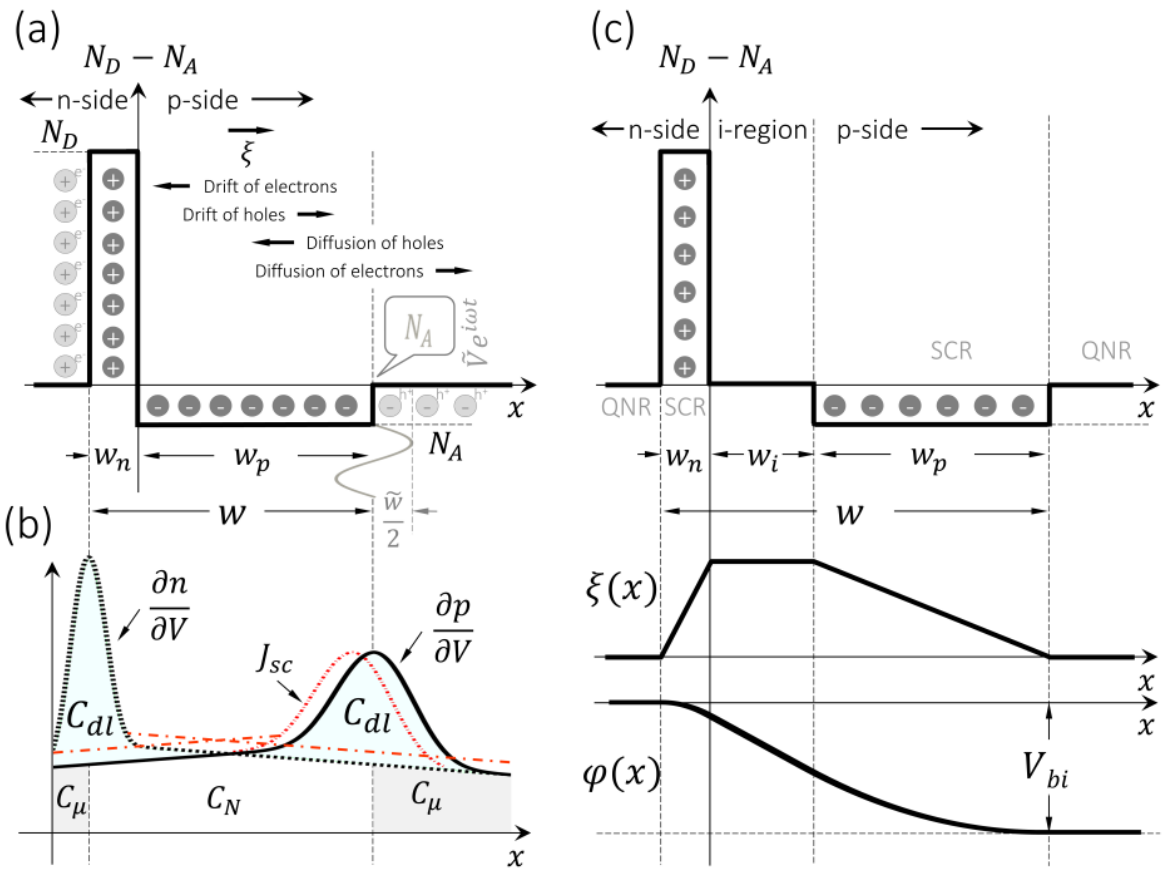

Figure 2.10. Abrupt one-sided homojunction (a) charge density profile and (b) corresponding $V$-mobile charge carriers. In (c) there is the analogue p-i-n profile and respective field and electrostatic potential. Filled circles with "+" and "-" symbols are fixed ionized donor and acceptor defects, respectively. Empty circles are neutral ions with their respective electron $\mathrm{e}^{+}$or hole $\mathrm{h}^{+}$.

Finally, if valid the conditions to apply (2.44) and (2.46) in the appropriate range where $L_{D}<$ $w<d$, then $C^{-2}$ may behave linear as

$$
C^{-2}=\frac{2}{q \varepsilon_{0} \varepsilon N}\left(V_{b i}-V\right)
$$

These are the main ideas behind the Mott-Schottky (MS) capacitance analysis, where the slope and intercepts of the MS plot inform on the effective values of $N$ and $V_{b i}$, respectively. Equation (2.49) can be properly modified to satisfy different density profiles, as summarized in Table 2.1.

The $\mathrm{p}-\mathrm{i}-\mathrm{n}$ junction case is of particular interest in PSCs and organic solar cells. In this profile, an intrinsic region of width $w_{i}$ lies between the two poles of the space charge region, as in Figure 
2.10c. Also, the electric field and Galvani potentials show constant and linear monotonic behaviors in the intrinsic region, as respectively illustrated in Figure 2.10c lower panel.

The energy stored in the electrostatic field can be found integrating (2.35) and substituting the expressions in Table 2.1. For instance, in the OSA p-n homojunction:

$$
E_{d l}=\sqrt{\frac{2 \varepsilon_{0} \varepsilon q N V_{b i}{ }^{3}}{9}}\left(\left(1-\frac{V}{V_{b i}}\right)^{\frac{3}{2}}\left(1-\frac{3 V_{b i}}{\left(V_{b i}-V\right)}\right)+2\right)
$$

which is a very low energy (see Section 2.6.3), in comparison with that extracted per second from a typical solar cell under standard 1 sun illumination.

Table 2.1. Different charge density profile models and respective expressions.

\begin{tabular}{|c|c|c|}
\hline Model & Depletion layer width & Capacitance MS analysis \\
\hline $\begin{array}{c}\text { Abrupt } \mathrm{p}-\mathrm{n} \\
\text { homojunction }^{[120]}\end{array}$ & $w=\sqrt{\frac{2 \varepsilon_{0} \varepsilon\left(N_{A}+N_{D}\right)}{q N_{A} N_{D}}}\left(V_{b i}-V-2 V_{t h}\right)$ & $C^{-2}=\frac{2\left(N_{A}+N_{D}\right)}{q \varepsilon_{0} \varepsilon N_{A} N_{D}}\left(V_{b i}-V-2 V_{t h}\right)$ \\
\hline $\begin{array}{l}\text { OSA p-n junction } \\
\qquad\left(N_{A} \ll N_{D}\right)^{[121]}\end{array}$ & $w \cong \sqrt{\frac{2 \varepsilon_{0} \varepsilon}{q N_{A}}}\left(V_{b i}-V\right)$ & $C^{-2} \cong \frac{2\left(V_{b i}-V\right)}{q \varepsilon_{0} \varepsilon N_{A}}$ \\
\hline Abrupt p-n heterojunction ${ }^{[9]}$ & $w=\sqrt{\frac{2 \varepsilon_{0} \varepsilon_{A} \varepsilon_{D}\left(N_{A}+N_{D}\right)^{2}}{q N_{A} N_{D}\left(N_{A} \varepsilon_{A}+N_{D} \varepsilon_{D}\right)}\left(V_{b i}-V\right)}$ & $C^{-2}=\frac{2\left(N_{A} \varepsilon_{A}+N_{D} \varepsilon_{D}\right)}{q \varepsilon_{0} N_{A} \varepsilon_{A} N_{D} \varepsilon_{D}}\left(V_{b i}-V\right)$ \\
\hline $\begin{array}{l}\text { OSA p-i-n homojunction } \\
\qquad\left(N_{A} \ll N_{D}\right)^{[121]}\end{array}$ & $w=\sqrt{\frac{2 \varepsilon_{0} \varepsilon}{q N_{A}}\left(V_{b i}-V\right)+w_{i}^{2}}$ & $C^{-2} \cong \frac{2\left(V_{b i}-V\right)}{q \varepsilon_{0} \varepsilon N_{A}}+\left(\frac{w_{i}}{\varepsilon \varepsilon_{0}}\right)^{2}$ \\
\hline $\begin{array}{c}\text { Abrupt } \\
\text { p-i-n homojunction }\end{array}$ & $w=\sqrt{\frac{2 \varepsilon_{0} \varepsilon\left(N_{A}+N_{D}\right)}{q N_{A} N_{D}}\left(V_{b i}-V\right)+w_{i}^{2}}$ & $C^{-2}=\frac{2\left(N_{A}+N_{D}\right)}{q \varepsilon_{0} \varepsilon N_{A} N_{D}}\left(V_{b i}-V\right)+\left(\frac{w_{i}}{\varepsilon \varepsilon_{0}}\right)^{2}$ \\
\hline $\begin{array}{l}\text { Linearly graded } \\
\text { p-n homojunction }{ }^{[121]}\end{array}$ & $w=\sqrt[3]{\frac{12 \varepsilon_{0} \varepsilon}{q a_{\rho}}\left(V_{b i}-V\right)}$ & $C^{-3}=\frac{12\left(V_{b i}-V\right)}{q a_{\rho} \varepsilon_{0}^{2} \varepsilon^{2}}$ \\
\hline $\begin{array}{l}\text { Arbitrary doping profile } \\
\text { p-n homojunction }\end{array}$ & $w=\frac{\varepsilon_{0} \varepsilon}{C}$ & $N(w)=-\frac{2}{q \varepsilon_{0} \varepsilon}\left(\frac{d\left(C^{-2}\right)}{d V}\right)^{-1}$ \\
\hline
\end{tabular}

"A" and "D" stand refereeing to the acceptor or donor side of the junction, and $\alpha_{\rho}$ is the doping concentration gradient.

Under illumination, typical silicon junctions behave not so different to the above concepts. For instance, at short-circuit the photo-junction capacitance results

$$
C_{s c} \cong C_{d l}\left(1+\frac{J_{s c}}{J_{\delta}}\right)
$$

where $C_{d l}$ is the dark depletion capacitance and usually $J_{\delta} \gg J_{s c}$ resulting $C_{s c} / C_{d l}<2$ under 1 sun illumination. ${ }^{[123-126]}$ In the case of p-i-n junctions, it has been proposed that $J_{\delta} \cong$ $2 V_{b i}{ }^{2}\left(\mu_{n}+\mu_{p}\right) w_{i}{ }^{-2} \cdot{ }^{[127]}$ Therefore, at SC, the photo-generation of charge carriers may lightly shrink the depletion width and enhance bulk densities in a way that the integral (2.41) is not significantly different. This is illustrated in Figure $2.10 \mathrm{~b}$ with red dot-dashed lines (assuming logarithmic ordinate axis).

The measurement of $C_{d l}$ is typically done via IS at a single high frequency, while sweeping the DC bias. Further experimental details will be provided in the next chapter. Also, the textbook example of linearly graded homojunction $\rho(x) \propto x$ was included in in Table 2.1 for illustrative 
reasons, and further studies on higher grading coefficients $M_{\rho}$ in the charge density profile $\rho(x) \propto$ $x^{M_{\rho}}$ can be found in the literature. ${ }^{[128-129]}$

In closing, the MS analysis results an useful tool for evaluating the charge density profiles, doping densities and built-in field in solar cells where the charge at the edge of the depletion layer can be modulated and sensed.

\subsubsection{Depletion capacitance II: Deep trap levels}

In the previous section, the depletion capacitance was introduced as a consequence of the formation of the rectifying junction with space charge regions, being $N_{A}$ and/or $N_{D}$ determining factors for $w$ and hence $C_{d l}$. The meaning and consequent naming of the acceptor and donor concentrations as "doping" densities is very straightforward in first generation silicon (group IV) devices. There, the conductivity type of each side of the junction is achieved by introducing interstitial and/or substitutional impurities in the crystal from groups III and V, typically B and $\mathrm{P}$, to produce $\mathrm{p}$ - and n-semiconductor, respectively. This doping creates allowed shallow levels in the bandgap close to the valence or conduction band due to the extrinsic acceptor and donor defects, respectively. Then the Fermi level is shifted from the intrinsic level towards the doping level.

Differently, like in thin film solar cells and the selective contacts in other emerging PVs, the conductivity can be achieved by a sort of "self-doping" due to intrinsic defects. Typical intrinsic point defects like vacancies, self-interstitials and antisite are always present, energetically distributed within the bandgap. Thus, only high enough concentration of shallow intrinsic defects, capable of shifting the Fermi level, permits to "self-dope" the semiconductor.

However, independently of the intrinsic or extrinsic nature of the defects, other deeper defects in lower concentrations $N_{t}$ can coexist with those responsible for the doping levels, $N_{A}$ or $N_{D}$. These so-called deep trap levels are of particular importance in the non-radiative SRH recombination processes, mainly if lying around mid-bandgap.

A deep trap acceptor single level with energy $E_{t}$ above the valence band and defect concentration $N_{t}$ is depicted in the energy diagram and respective charge density profile of the pside of a junction in Figure 2.11. There, the $q V_{b i}$ band bending within the space charge region $w$ is illustrated, given that the intrinsic level "follows" the electrostatic potential and the acceptor defects below the Fermi level may be ionized.

The presence of the trap level makes an step in the charge density profile $\rho(x)$ at position $x_{t}$ where $E_{F}(x)=E_{t}(x)$, as in the middle panel of Figure 2.11. Note that, in the schemed region towards the junction, only at $x_{t}$ and $w$ it is possible to obtain non-zero values from the time derivative of $\rho(x)$ under an AC voltage perturbation, as in the lower panel of Figure 2.11.

Unfortunately, in-situ evaluation of $x_{t}$ is unpractical due to experimental limitations, and also typically $N_{t}$ is much lower than the doping concentration. Hence, it is intuitive to think that when changing voltage, the current signal (in transient or AC experiments) provides information from both $w$ and $x_{t}$, respectively the doping and deep trap level. However, deep traps and doping levels not only contribute to current at different points in the space charge region, but, more importantly, they have well defined different characteristic response time.

Illustratively, for an acceptor trap in equilibrium like Figure 2.11 the increase of applied reverse bias boost the relative distance between the $E_{F}$ and $E_{V}$ in the band bending region. Thus, 
$w$ and $x_{t}$ increase as defects are ionized. The trapping-detrapping processes at $x_{t}$ due to voltage excitation have a characteristic time $\tau=\omega_{e m}{ }^{-1}$, which also characterize the detailed balanced thermal capture-emission processes. Assuming no degeneracy, the emission rate results

$$
\omega_{e m}=N_{V} v_{t h} \sigma_{c s} \exp \left[-\frac{E_{t}}{k_{B} T}\right]
$$

with $N_{V}$ after (2.3), $v_{t h}=\sqrt{2 k_{B} T / \pi m_{h}^{*}}$ is the holes thermal velocity, $\sigma_{c S}$ the capture cross section and $\omega_{0}=N_{V} v_{t h} \sigma_{c S}$ the so called attempt-to-escape frequency. The latter can be found assuming thermal independent $\sigma_{c s}$ as

$$
\omega_{0}=\frac{8 \pi M_{V} m_{h}^{*} \sigma_{c s} k_{B}^{2}}{h^{3}} T^{2}
$$

which is a quadratic function of temperature.

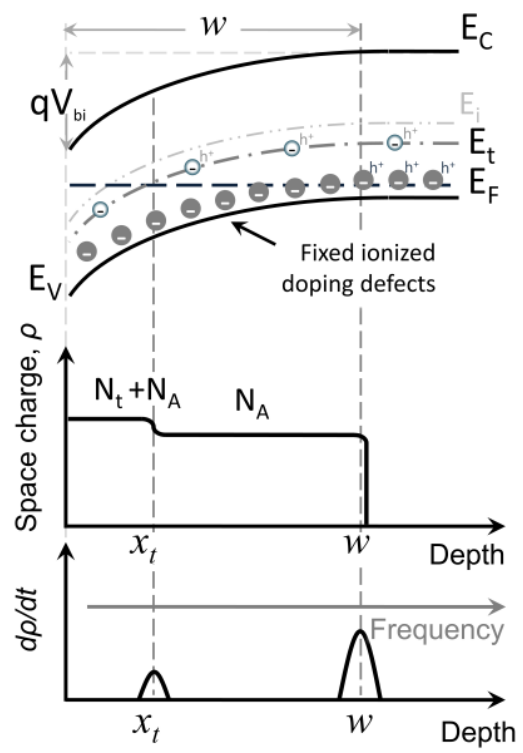

Figure 2.11. Equilibrium energy diagram of the p-side band bending in an abrupt junction with a bulk deep defect level of energy $E_{t}$ above the valence band and defect concentration $N_{t}$. Below panels show the corresponding charge density profile $\rho(x)$ and space charge time variations $\frac{d \rho(x)}{d t} \quad$ upon voltage perturbation at each point of the region towards the junction. A stronger signal is typically sensed from the edge of the depletion zone than from traps inside the space charge region. The frequency domain is an useful tool to solve each contribution to the total depletion layer capacitance.

The time and temperature dependencies of the traps emission/capture processes differentiates distinct contributions to the charging/discharging of the depletion region, and hence capacitance. Note that the shallower $E_{t}$ is the higher $\omega_{e m}$, at a given $T$. Later, for a deeper enough level $E_{t}$ the corresponding signal may evolve with temperature being the exponential factor in (2.52) predominant, so $\omega_{e m}$ decreases as $T$ does.

To sum up, significant concentration of trap levels $N_{t}$, with deeper energy distance $E_{t}$ from the bands, modifies the depletion layer capacitance in different time/frequency scales than the $C_{d l}$ defining doping concentrations. Besides, the characteristic emission rate of these processes is 
temperature dependent. These are the main concepts behind several characterization techniques like deep level transient spectroscopy (DLTS) and thermal admittance spectroscopy (TAS).

\subsubsection{Forward bias capacitance}

In the two previous sections, the doping and deep level defects capacitive features from classical p-n semiconductor junctions were introduced for dark reverse bias regime, or low enough forward bias condition. These are situations where the DC current is expected to be negligible or not far from the order of the displacement currents upon transient or AC perturbation experiments. Even under illumination, within these voltage ranges the typical silicon solar cells respond nearly the same when a DC photocurrent is flowing. ${ }^{[123-124,126]}$

However, as forward bias approaches $V_{b i}$ the space charge region shrinks up to the limit of the Debye length and the minority carriers concentrations are enhanced along the device, approaching the majority concentrations. Then, injection makes the bulk mobile carrier concentration (see Figure 2.10b) to overlap and exceed the dipolar configuration of $C_{d l}$. The charge from the enhanced QNR may contribute to the integral (2.41), that can be rewritten as the chemical capacitance per unit area ${ }^{[115]}$

$$
C_{\mu}=q \int_{0}^{d} \frac{1}{n+p} \frac{\partial(n p)}{\partial V} d x
$$

which for the OSA p-n homojunction $\left(N_{A} \ll N_{D}\right)$, in dark, results as ${ }^{[115,130]}$

$$
C_{\mu}=\frac{n_{i}^{2} q^{2}}{m_{C} k_{B} T} \frac{L_{n}}{N_{A}} \exp \left[\frac{q V}{k_{B} T}\right]
$$

being $m_{C}$ a capacitance ideality factor equaling unity at higher voltages and the capacitance contributions concentrated within the diffusion length. ${ }^{[130]}$ This is the reason why $C_{\mu}$ is also known as diffusion capacitance. Note that from (2.21) and (2.54a) we obtain the proportionality with the forward bias current from the dark Shockley equation: ${ }^{[131]}$

$$
C_{\mu}=\frac{q \tau_{n}}{m_{C} k_{B} T} J_{S} \exp \left[\frac{q V}{k_{B} T}\right]
$$

Equation (2.54b) has been empirically adapted to OC under illumination condition by substituting $J_{S}$ by the corresponding $J_{S c}{ }^{[131]}$ However, conceptually this is not correct, due to conflicting $\tau_{n}$ definition (2.22) and the integral limits in (2.53). At OC under illumination a forward bias $V=V_{o c}$ should be applied to cancel DC currents. Hence, the chemical potentials $E_{F n}$ and $E_{F p}$ are nearly flat $\left(\partial E_{F n} / \partial x \cong \partial E_{F p} / \partial x \cong 0\right)$, if low field effects are considered. This situation transforms $(2.8)$ to

$$
q V_{o c} \cong E_{F n}-E_{F n}
$$

which can be derived to rewrite definition $(2.53)$ as $^{[42,132]}$

$$
C_{\mu}=q^{2} \bar{d} \frac{\partial n}{\partial E_{F n}}
$$

Subsequently, by deriving (2.9) with the neutrality condition we get ${ }^{[126,133-134]}$

$$
C_{\mu}=\frac{q^{2} d}{2 k_{B} T} n_{i}^{2} \exp \left[\frac{E_{F n}-E_{F p}}{k_{B} T}\right]\left(\left(\frac{N_{D}-N_{A}}{2}\right)^{2}+n_{i}^{2} \exp \left[\frac{E_{F n}-E_{F p}}{k_{B} T}\right]\right)^{-1 / 2}
$$


At high voltages low voltages (2.57) indicates $C_{\mu} \propto \exp \left[q V / 2 k_{B} T\right]$, but at low bias (2.57) is different to (2.54) only by the ratio $L_{n} / d$. An analogue result is obtained if taking $\partial p / \partial E_{F p}$ in (2.56). More importantly, note that (2.57) and (2.56) correspond to the chemical capacitance of the occupation of single states, i.e. $\mathrm{CB}$ or $\mathrm{VB}$, which in abrupt bandgaps are defining the currents. In systems with a broad density of states (DOS, $g$ ) it can be assumed that $C_{\mu} \approx q^{2} L g\left(E_{F n}\right)$. ${ }^{[2]}$

It is convenient to empirically combine (2.54) and (2.57) as

$$
C_{\mu}=\frac{q^{2}}{k_{B} T} \frac{n_{\mu} L_{\mu}}{m_{\mu}} \exp \left[\frac{q V}{m_{\mu} k_{B} T}\right]
$$

where $n_{\mu} \approx n_{i}{ }^{2} /\left(N_{A}+N_{D}\right)$ is the storage carrier density contributing to $C_{\mu}, L_{\mu}$ is the effective chemical capacitance integration length $\left(L_{\mu} \leq L\right)$, and $1 \leq \mathrm{m}_{\mu}(\mathrm{V}) \leq 2$ is the DC bias dependent chemical capacitance ideality factor.

The energy which is stored in the bulk material, regardless of the electric field, can be found by substituting (2.58) in (2.35) and integrating, so

$$
E_{\mu} \cong n_{\mu} L_{\mu} q V \exp \left[\frac{q V}{m_{\mu} k_{B} T}\right]
$$

The capacitance $C_{\mu}$ and energy $E_{\mu}$ as a function of the storage density $n_{\mu}$ are presented in Figure 2.12 for a typical Si solar cell in OC situation under standard 1 sun illumination. Also in Figure 2.12, the depletion capacitance as a function of the doping density is displayed in a situation close to $V_{b i}$ (highest $C_{d l}$ values). It is evident that $C_{\mu}$ exceeds $C_{d l}$ several orders of magnitude as $V_{b i}$ is approached and surpassed.

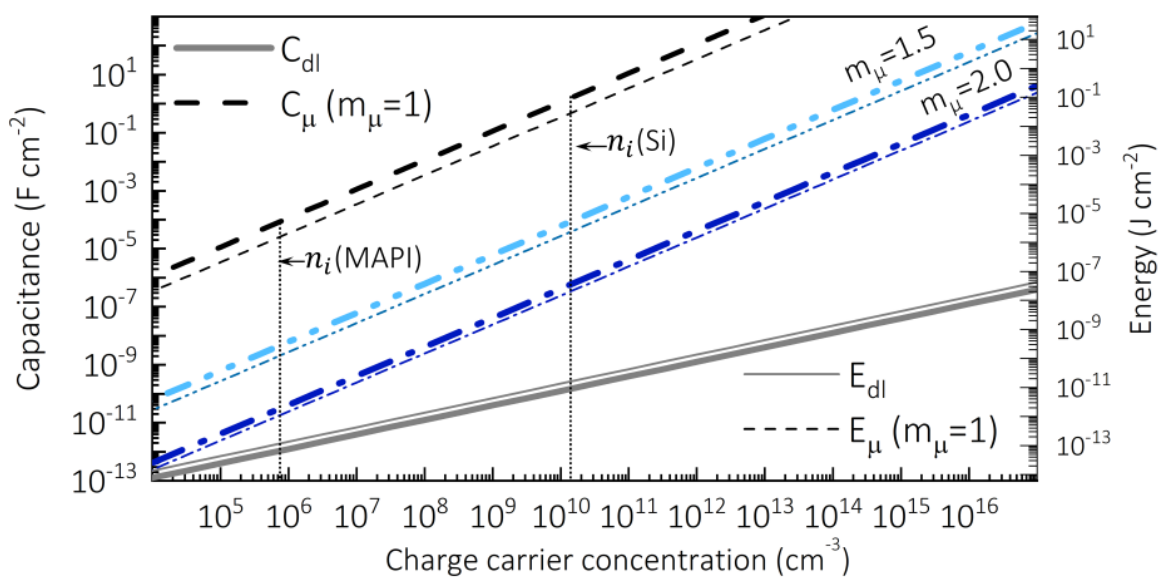

Figure 2.12. Capacitance (left) and energy (right) as a function of the doping concentration $N$, for the depletion capacitance, and storage density $N_{\mu}$, for chemical capacitance. Simulation was made for a Si solar cell at $T=300 \mathrm{~K}$ with $\varepsilon=11.3, V_{b i}=0.7 \mathrm{~V}$ and $V=$ $650 \mathrm{mV}$ in (2.49), (2.50), and $L_{\mu}=100 \mathrm{~nm}, V_{o c}=730 \mathrm{mV}$ and $m_{\mu}=1 ; 1.5 ; 2$ in (2.58) and (2.59). 
Three illustrative values for $\mathrm{m}_{\mu}$ are simulated, showing unrealistically larger results for $m_{\mu}=$ 1 when $n_{\mu}>10^{10} \mathrm{~cm}^{-3}$. This suggest that a transition to $\mathrm{m}_{\mu}=2$ is most likely expected as forward bias increases, similarly to (2.57), since $n_{i} \approx 10^{10} \mathrm{~cm}^{-3}$ for Si at room temperature. Interestingly, despite unviable in traditional $\mathrm{Si}$ solar cells, $E_{\mu}$ could reach significant values comparable with supercapacitor behavior if a given modification to the device would make $m_{\mu} \approx$ 2 and $n_{\mu} \gg n_{i}$.

In summary, at forward bias an exponential increase of the chemical capacitance is the main mechanism of bulk charge accumulation in a non-dipolar/non-dielectric way.

\subsection{Perovskite solar cells and supercapacitors}

The combination of the concepts of energy generation and storage has gained the attention of some research groups. ${ }^{[61]}$ Here the supercapacitors seem like a good option. ${ }^{[135-143]}$ This would be advantageous as a supplementary energy storage element in stand-alone and hybrid photovoltaic systems, as well as for some applications like wearable devices and building integration. Furthermore,

There have been three main designs: the horizontally packed, and the parallel and series vertically stacked configurations, as schemes in Figure 2.13a-c. In the horizontal packing variant, both individual devices are laterally set on the same substrate and wired through the opposite electrode. ${ }^{[135]}$ In the parallel vertically stacked alternative, one of the two devices is set on top of the other. ${ }^{[136,138-139]}$ Either way, the cell charges the supercapacitor connected in parallel through the photovoltage $V_{o c}$ created during illumination. The corresponding AC equivalent circuit is in Figure 2.13d.

(a)

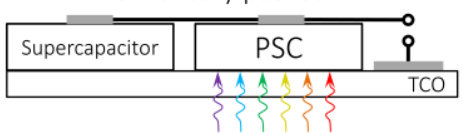

(b)

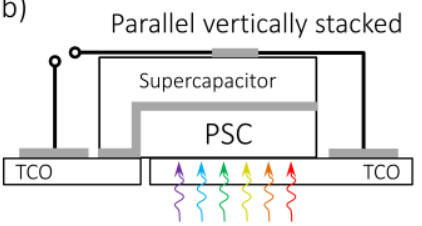

(c)

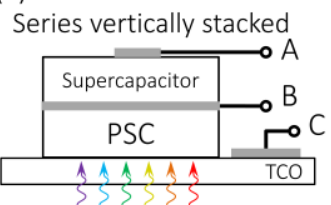

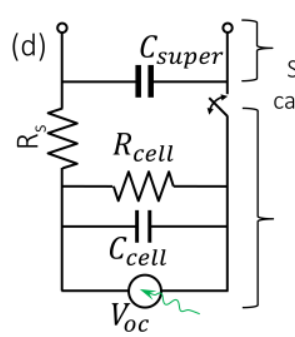

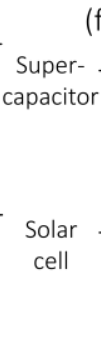

(f)

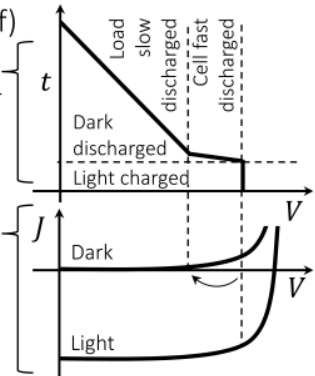

(g)

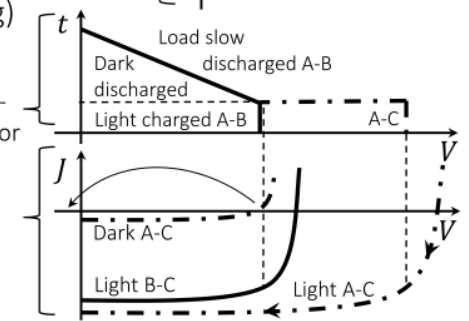

Figure 2.13. Supercapacitors and PSCs integration concept. Schemed configurations as (a) horizontally packed and (b)vertically stacked. In (c), the respective AC equivalent circuit 
and in (d) the voltage time evolution in the supercapacitor as the current-voltage curve transits from light operation to dark in the solar cell. TCO is transparent conducting oxide.

In series vertical stacked design, the cell and the supercapacitor are no longer at the same voltage and three points $\mathrm{A}, \mathrm{B}$ and $\mathrm{C}$ are defined in structure scheme and equivalent circuit of Figure $2.13 \mathrm{c}$,e, respectively. ${ }^{[139]}$

For the parallel configurations, during light operation the cell first charges the capacitor and then keeps the charged state at operational voltage while the power is consumed by the load. Figure 2.13f shows when light is set off. Then the cell first discharge fast the supercapacitor in the region where it is nearly short-circuiting. Subsequently, the large resistance of the cell makes the discharging slower, as dominated by the load. In this configuration, the wired electrodes can be also disconnected via a switcher, so there is no discharge through the cell in dark.

For the series configuration, similarly, during light operation the supercapacitor (A-B) is charged by the cell (B-C). The voltage at the supercapacitor can be very close to that of the $V_{o c}$, depending of the load. A fast FR voltage sweep between points $\mathrm{A}$ and $C$ may deliver an enhanced efficiency, since the effective $V_{o c}$ can be nearly twice that of the cell, and the $J_{c a p}$ may add extra $J_{s c}$. In practice, the operational point will be somewhere between the $J-V$ sweep from A-C and the actual cell B-C. An equilibrium should be stated between the battery charging/discharging and the load. Furthermore, when light is switched off, the cell may only affect the voltage decay of the capacitor via the ohmic energy dissipation.

Interestingly, an $\mathrm{Au} / \mathrm{MAPI} / \mathrm{Au}$ supercapacitor with fast discharge has been reported by Slonopas et al. ${ }^{[144]}$ At room temperature, the device exhibited an energy density of $34.2 \mathrm{Wh} \cdot \mathrm{kg}^{-1}$ at $100 \mathrm{~Hz}$, a mean capacitance above $400 \mathrm{mF} \cdot \mathrm{cm}^{-2}$ between $100 \mathrm{~Hz}$ and $100 \mathrm{kHz}$ and the capability of operating at potential differences of up to $10 \mathrm{~V}$.

In summary, the combination of power generation by photovoltaic PSCs and energy storage in integrated external supercapacitors has been reported. Here the question arises on whether it would be possible to enhance the capacitance of the cell itself for creating a supercapacitive perovskite solar cell.

\section{References}

[1] M. A. Green, Solar Cells. Operating principles, Technology and System Applications, Prentice-Hall, 1982. ISBN: 0858235803.

[2] J. Bisquert, The Physics of Solar Cells: Perovskites, Organics, and Photovoltaic, CRC Press, Boca Raton, 2018. ISBN: 9781138099968.

[3] J. Nelson, The Physics of Solar Cells, Imperial College Press, Singapore, 2003. ISBN: 1860943403.

[4] A. L. Fahrenbruch, R. H. Bube, Fundamentals of Solar Cells. Photovoltaic Solar Energy Conversion, Academic Press, New York, 1983. ISBN: 0122476808.

[5] P. Würfel, Physics of Solar Cells: From Principles to New Concepts, WILEY-VCH Verlag GmbH \& Co. KGaA, Weinheim, 2005. ISBN: 3527404287.

[6] M. Freitag et al., Nat. Photon. 11 (2017), 372. https://doi.org/10.1038/nphoton.2017.60

[7] C. Kittel, Introduction to Solid States Physics John Wiley \& Sons, USA, 2005. ISBN: 9780471415268.

[8] N. W. Ashcroft, N. D. Mermin, Solid State Physics, Harcourt, USA, 1976. ISBN: 0030839939.

[9] S. M. Sze, K. K. Ng, Physics of Semiconductor Devices, John Wiley \& Sons, Hoboken, New Jersey, USA, 2007. ISBN: 9780471143239. 
[10] R. F. Pierret, Semiconductor Device Fundamentals, Addison-Wesley Pub. Com., USA, 1996. ISBN: 0201543931.

[11] J. E. Parrott, Sol. Energy Mater. Sol. Cells 30 (1993), 221. https://doi.org/10.1016/09270248(93)90142-P

[12] L. M. Pazos-Outón et al., Science 351 (2016), 1430. https://doi.org/10.1126/science.aaf1168

[13] W. Shockley, W. T. Read, Phys. Rev. 87 (1952), 835. https://doi.org/10.1103/PhysRev.87.835

[14] R. N. Hall, Phys. Rev. 87 (1952), 387. https://doi.org/10.1103/PhysRev.87.387

[15] W. Shockley, H. J. Queisser, J. Appl. Phys. 32 (1961), 510. https://doi.org/10.1063/1.1736034

[16] S. Rühle, Solar Energy 130 (2016), 139. https://doi.org/10.1016/j.solener.2016.02.015

[17] O. Almora, L. Vaillant-Roca, G. Garcia-Belmonte, Rev. Cubana Fis. 34 (2017), 58.

http://www.revistacubanadefisica.org/index.php/rcf/article/view/RCF_34-1_58

[18] Y. Bai, X. Meng, S. Yang, Adv. Energy Mater. 8 (2018), 1701883.

https://doi.org/10.1002/aenm.201701883

[19] K. Choi et al., Solar RRL n/a (2019). https://doi.org/10.1002/solr.201900251

[20] A. a. O. El-Ballouli, O. M. Bakr, O. F. Mohammed, Chem. Mater. 31 (2019), 6387. https://doi.org/10.1021/acs.chemmater.9b01268

[21] Z. Yang et al., Solar RRL n/a (2019). https://doi.org/10.1002/solr.201900257

[22] P. Wang et al., Res. Chem. Intermed. 42 (2016), 625. https://doi.org/10.1007/s11164-015-2046-x

[23] A. M. Al-Amri, B. Cheng, J. He, IEEE Trans. Nanotech. 18 (2019), 1. https://doi.org/10.1109/TNANO.2018.2872887

[24] Z. Zhao et al., J. Mater. Chem. A 5 (2017), 4756. https://doi.org/10.1039/C6TA10305G

[25] Z. Zhou, S. Pang, Z. Liu, H. Xu, G. Cui, J. Mater. Chem. A 3 (2015), 19205.

https://doi.org/10.1039/C5TA04340A

[26] P. Schulz, E. Edri, S. Kirmayer, G. Hodes, D. Cahen, A. Kahn, Energy Environ. Sci. 7 (2014), 1377. https://doi.org/10.1039/C4EE00168K

[27] A. Imanishi, E. Tsuji, Y. Nakato, J. Phys. Chem. C 111 (2007), 2128.

[28] P. Qin et al., Nanoscale 6 (2014), 1508. https://doi.org/10.1039/C3NR05884K

[29] G. Y. Margulis et al., Adv. Energy Mater. 3 (2013), 1657. https://doi.org/10.1002/aenm.201300660

[30] N. D. Orf, I. D. Baikie, O. Shapira, Y. Fink, Appl. Phys. Lett. 94 (2009), 113504.

https://doi.org/10.1063/1.3089677

[31] X. Liu et al., Phy. Chem. Chem. Phys. 17 (2015), 896. https://doi.org/10.1039/C4CP03842H

[32] H. B. Michaelson, J. Appl. Phys. 48 (1977), 4729. https://doi.org/10.1063/1.323539

[33] R. A. Hatton, N. P. Blanchard, L. W. Tan, G. Latini, F. Cacialli, S. R. P. Silva, Org. Electron. 10 (2009), 388. https://doi.org/10.1016/j.orgel.2008.12.013

[34] Y. Kim, A. M. Ballantyne, J. Nelson, D. D. C. Bradley, Org. Electron. 10 (2009), 205. https://doi.org/10.1016/j.orgel.2008.10.003

[35] Y. Zhou et al., Appl. Phys. Lett. 92 (2008), 233308. https://doi.org/10.1063/1.2945796

[36] M.-C. Wu et al., Chem. Phys. Lett. 468 (2009), 64. https://doi.org/10.1016/j.cplett.2008.11.080

[37] Z. Wang, U. Helmersson, P.-O. Käll, Thin Solid Films 405 (2002), 50. https://doi.org/10.1016/S0040-6090(01)01767-9

[38] L. Martinu, D. Poitras, J. Vac. Sci. Technol., A 18 (2000), 2619. https://doi.org/10.1116/1.1314395

[39] A. Janotti, J. B. Varley, J. L. Lyons, C. G. Van de Walle, in Functional Metal Oxide

Nanostructures, Springer, New York, USA 2012, 23. https://doi.org/10.1007/978-1-4419-9931-3_2

[40] B. S. Jeong, D. P. Norton, J. D. Budai, Solid-State Electron. 47 (2003), 2275.

https://doi.org/10.1016/S0038-1101(03)00210-7

[41] A. Guerrero, E. J. Juarez-Perez, J. Bisquert, I. Mora-Sero, G. Garcia-Belmonte, App. Phys. Lett. 105 (2014), 133902. https://doi.org/10.1063/1.4896779

[42] J. Bisquert, Nanostructured Energy Devices: Equilibrium Concepts and Kinetics, CRC Press Taylor \& Francis Group, Boca Raton, 2014. ISBN: 9781439836026.

[43] Y. Bai, I. Mora-Seró, F. De Angelis, J. Bisquert, P. Wang, Chem. Rev. 114 (2014), 10095. https://doi.org/10.1021/cr400606n

[44] D. V. Bavykin, J. M. Friedrich, F. C. Walsh, Adv. Mater. 18 (2006), 2807. https://doi.org/10.1002/adma.200502696

[45] Y. Dai, C. M. Cobley, J. Zeng, Y. Sun, Y. Xia, Nano Letters 9 (2009), 2455.

https://doi.org/10.1021/n1901181n 
[46] M. He, D. Zheng, M. Wang, C. Lin, Z. Lin, J. Mater. Chem. A 2 (2014), 5994. https://doi.org/10.1039/C3TA14160H

[47] K. Akaike et al., J. Appl. Phys. 104 (2008), 023710. https://doi.org/10.1063/1.2957588

[48] F. Zhang et al., Sol. Energy Mater. Sol. Cells 97 (2012), 71.

https://doi.org/10.1016/j.solmat.2011.09.006

[49] A. A. Y. Guilbert, M. Zbiri, A. D. F. Dunbar, J. Nelson, J. Phys. Chem. B 121 (2017), 9073. https://doi.org/10.1021/acs.jpcb.7b08312

[50] J. Lian, B. Lu, F. Niu, P. Zeng, X. Zhan, Small Methods 2 (2018), 1800082. https://doi.org/10.1002/smtd.201800082

[51] G.-P. Mao et al., Rare Metals 37 (2018), 95. https://doi.org/10.1007/s12598-017-0951-4

[52] S. Fantacci, F. De Angelis, M. K. Nazeeruddin, M. Grätzel, J. Phys. Chem. C 115 (2011), 23126. https://doi.org/10.1021/jp207968b

[53] Y. Liu et al., Nano Lett. 15 (2015), 662. https://doi.org/10.1021/nl504168q

[54] R. Schölin, M. H. Karlsson, S. K. Eriksson, H. Siegbahn, E. M. J. Johansson, H. Rensmo, J. Phys. Chem. C 116 (2012), 26300. https://doi.org/10.1021/jp306433g

[55] Q. Pei, G. Zuccarello, M. Ahlskog, O. Inganäs, Polymer 35 (1994), 1347. https://doi.org/10.1016/0032-3861(94)90332-8

[56] G. Greczynski, T. Kugler, M. Keil, W. Osikowicz, M. Fahlman, W. R. Salaneck, J. Electron. Spectrosc. Relat. Phenom. 121 (2001), 1. https://doi.org/10.1016/S0368-2048(01)00323-1

[57] X. Yang, H. Wang, B. Cai, Z. Yu, L. Sun, J. Energy Chem. 27 (2018), 650. https://doi.org/10.1016/j.jechem.2017.12.017

[58] R. Singh, P. K. Singh, B. Bhattacharya, H.-W. Rhee, Appl. Mater. Today 14 (2019), 175. https://doi.org/10.1016/j.apmt.2018.12.011

[59] S. A. Veldhuis et al., Adv. Mater. 28 (2016), 6804. https://doi.org/10.1002/adma.201600669

[60] M. M. Stylianakis, T. Maksudov, A. Panagiotopoulos, G. Kakavelakis, K. Petridis, Materials 12 (2019), 859. https://doi.org/10.3390/ma12060859

[61] W. Zhang, G. E. Eperon, H. J. Snaith, Nat. Energy 1 (2016), 16048.

https://doi.org/10.1038/nenergy.2016.48

[62] O. Almora, C. J. Brabec, (2020).

[63] T. Baikie et al., J. Mater. Chem. A 1 (2013), 5628. https://doi.org/10.1039/C3TA10518K

[64] Y. Yasuhiro, N. Toru, E. Masaru, W. Atsushi, K. Yoshihiko, Appl. Phys. Express 7 (2014), 032302. https://doi.org/10.7567/APEX.7.032302

[65] N.-G. Park, M. Grätzel, T. Miyasaka, Eds., Organic-Inorganic Halide Perovskite Photovoltaics: From Fundamentals to Device Architectures, Springer, Switzerland 2016. ISBN: 9783319351148.

[66] T.-B. Song et al., J. Mater. Chem. A 3 (2015), 9032. https://doi.org/10.1039/C4TA05246C

[67] Y. Wang, Y. Zhang, P. Zhang, W. Zhang, Phys. Chem. Chem. Phys. 17 (2015), 11516. https://doi.org/10.1039/C5CP00448A

[68] C. C. Stoumpos, C. D. Malliakas, M. G. Kanatzidis, Inorg. Chem. 52 (2013), 9019. https://doi.org/10.1021/ic401215x

[69] S. Luo, W. A. Daoud, J. Mater. Chem. A 3 (2015), 8992. https://doi.org/10.1039/C4TA04953E

[70] J. Feng, B. Xiao, J. Phys. Chem. Lett. 5 (2014), 1278. https://doi.org/10.1021/jz500480m

[71] D. Song et al., J. Phys. Chem. C 119 (2015), 22812. https://doi.org/10.1021/acs.jpcc.5b06859

[72] J. Kim, S.-H. Lee, J. H. Lee, K.-H. Hong, J. Phys. Chem. Lett. 5 (2014), 1312 https://doi.org/10.1021/jz500370k

[73] T. Shi, W.-J. Yin, Y. Yan, J. Phys. Chem. C 118 (2014), 25350. https://doi.org/10.1021/jp508328u

[74] W.-J. Yin, T. Shi, Y. Yan, Appl. Phys. Lett. 104 (2014), 063903. https://doi.org/10.1063/1.4864778

[75] W.-J. Yin, T. Shi, Y. Yan, Adv. Mater. 26 (2014), 4653. https://doi.org/10.1002/adma.201306281

[76] Y. Yuan, J. Huang, Acc. Chem. Res. 49 (2016), 286. https://doi.org/10.1021/acs.accounts.5b00420

[77] C. Li, A. Guerrero, S. Huettner, J. Bisquert, Nat. Commun. 9 (2018), 5113.

https://doi.org/10.1038/s41467-018-07571-6

[78] J. Haruyama, K. Sodeyama, L. Han, Y. Tateyama, J. Am. Chem. Soc. 137 (2015), 10048. https://doi.org/10.1021/jacs.5b03615

[79] C. Eames, J. M. Frost, P. R. F. Barnes, B. C. O'Regan, A. Walsh, M. S. Islam, Nat. Commun. 6 (2015), 7497. https://doi.org/10.1038/ncomms8497 
[80] E. Mosconi, F. De Angelis, ACS Energy Lett. 1 (2016), 182.

https://doi.org/10.1021/acsenergylett.6b00108

[81] M. I. Asghar, J. Zhang, H. Wang, P. D. Lund, Renewable Sustainable Energy Rev. 77 (2017), 131. https://doi.org/10.1016/j.rser.2017.04.003

[82] U. Krishnan, M. Kaur, M. Kumar, A. Kumar, J. Photonics Energy 2 (2019), 021001. https://doi.org/10.1117/1.JPE.9.021001

[83] M. Shirayama et al., J. Appl. Phys. 119 (2016), 115501. https://doi.org/10.1063/1.4943638

[84] W. Huang, J. S. Manser, P. V. Kamat, S. Ptasinska, Chem. Mater. 28 (2016), 303. https://doi.org/10.1021/acs.chemmater.5b04122

[85] T. T. Ava, A. Al Mamun, S. Marsillac, G. Namkoong, Appl. Sci. 9 (2019). htps://doi.org/10.3390/app9010188

[86] N. Onoda-Yamamuro, T. Matsuo, H. Suga, J. Phys. Chem. Solids 53 (1992), 935. https://doi.org/10.1016/0022-3697(92)90121-S

[87] K. Domanski, E. A. Alharbi, A. Hagfeldt, M. Grätzel, W. Tress, Nat. Energy 3 (2018), 61. https://doi.org/10.1038/s41560-017-0060-5

[88] G. Niu, X. Guo, L. Wang, J. Mater. Chem. A 3 (2015), 8970.

https://doi.org/10.1039/C4TA04994B

[89] E. J. Juarez-Perez, L. K. Ono, M. Maeda, Y. Jiang, Z. Hawash, Y. Qi, J. Mater. Chem. A 6 (2018), 9604. https://doi.org/10.1039/C8TA03501F

[90] A. Guerrero et al., ACS Nano 10 (2016), 218. https://doi.org/10.1021/acsnano.5b03687

[91] J. Carrillo et al., Adv. Energy Mater. 6 (2016), 1502246 https://doi.org/10.1002/aenm.201502246

[92] C. C. Boyd, R. Cheacharoen, K. A. Bush, R. Prasanna, T. Leijtens, M. D. McGehee, ACS Energy Lett. 3 (2018), 1772. https://doi.org/10.1021/acsenergylett.8b00926

[93] P. Lopez-Varo et al., Adv. Energy Mater. 8 (2018), 1702772.

https://doi.org/10.1002/aenm.201702772

[94] D. F. Swinehart, J. Chem. Educ. 39 (1962), 333. https://doi.org/10.1021/ed039p333

[95] W. Tress, K. Leo, M. Riede, Phys. Rev. B 85 (2012), 155201.

https://doi.org/10.1103/PhysRevB.85.155201

[96] S. S. Li, in Semiconductor Physical Electronics, Springer, New York 2006, 134. https://doi.org/10.1007/0-387-37766-2_6

[97] R. E. I. Schropp, M. Zeman, in Amorphous and Microcrystalline Silicon Solar Cells: Modeling, Materials and Device Technology, Vol. 5, Springer, Boston 1998, 117. https://doi.org/10.1007/978-1-46155631-2_6

[98] W. Shockley, Bell Syst. Tech. J. 28 (1949), 435. https://doi.org/10.1002/j.15387305.1949.tb03645.x

[99] W. Shockley, Electrons and Holes in Semiconductors with Applications to Transistor Electronics, D. Van Nostrand, Princenton, New Jersey, 1950.

[100] M. Bashahu, P. Nkundabakura, Solar Energy 81 (2007), 856. https://doi.org/10.1016/j.solener.2006.11.002

[101] W. Tress et al., Energy Environ. Sci. 11 (2017), 151. https://doi.org/10.1039/C7EE02415K

[102] O. Almora et al., Nano Energy 48 (2018), 63. https://doi.org/10.1016/j.nanoen.2018.03.042

[103] H.-S. Kim, N.-G. Park, J. Phys. Chem. Lett. 5 (2014), 2927. https://doi.org/10.1021/jz501392m

[104] E. L. Unger et al., Energy Environ. Sci. 7 (2014), 3690. https://doi.org/10.1039/C4EE02465F

[105] H. J. Snaith et al., J. Phys. Chem. Lett. 5 (2014), 1511. https://doi.org/10.1021/jz500113x

[106] A. Edler, M. Schlemmer, J. Ranzmeyer, R. Harney, Energy Procedia 27 (2012), 267. https://doi.org/10.1016/j.egypro.2012.07.062

[107] Q. Gao, Y. Zhang, Y. Yu, F. Meng, Z. Liu, IEEE J. Photovoltaics 8 (2018), 710. https://doi.org/10.1109/JPHOTOV.2018.2810852

[108] A. Haque, K. V. S. Bharath, M. A. Khan, I. Khan, Z. A. Jaffery, Energy Sci. Eng. 7 (2019), 622. https://doi.org/10.1002/ese3.255

[109] L. Liu, H. Zhao, Y. Lei, Small Methods 3 (2019), 1800341. https://doi.org/10.1002/smtd.201800341

[110] E. Lim, C. Jo, J. Lee, Nanoscale 8 (2016), 7827. https://doi.org/10.1039/C6NR00796A

[111] C. G. Shuttle et al., App. Phys. Lett. 92 (2008), 093311. https://doi.org/10.1063/1.2891871

[112] J. W. Ryan, E. Palomares, Adv. Energy Mater. 7 (2017), 1601509.

https://doi.org/10.1002/aenm.201601509 
[113] N. W. Duffy, L. M. Peter, R. M. G. Rajapakse, K. G. U. Wijayantha, Electrochem. Commun. 2 (2000), 658. https://doi.org/10.1016/S1388-2481(00)00097-7

[114] D. L. Heald, P. F. Ordung, J. G. Skalnik, E. N. Nansen, Solid-State Electron. 16 (1973), 1055. https://doi.org/10.1016/0038-1101(73)90207-4

[115] J. E. Parrott, L. P. Leonidou, Phys. Stat. Sol. (a) 25 (1974), 231.

https://doi.org/10.1002/pssa.2210250121

[116] J. J. H. Van den Biesen, Philips J. Res. 40 (1985), 88.

[117] J. Heath, P. Zabierowski, in Advanced Characterization Techniques for Thin Film Solar Cells, Vol. 1 (Eds: D. Abou-Ras, T. Kirchartz, U. Rau), Wiley-VCH Verlag GmbH \& Co. KGaA, Boschstr. 12, 69469 Weinheim, Germany 2011, 81. https://doi.org/10.1002/9783527636280.ch4

[118] J. Werner, A. F. J. Levi, R. T. Tung, M. Anzlowar, M. Pinto, Phys. Rev. Lett. 60 (1988), 53. https://doi.org/10.1103/PhysRevLett.60.53

[119] J. J. H. Van Den Biesen, Philips J. Res. 40 (1985), 103.

[120] H. K. Gummel, D. L. Scharfetter, J. Appl. Phys. 38 (1967), 2148.

https://doi.org/10.1063/1.1709844

[121] C. van Opdorp, Solid-State Electron. 11 (1968), 397. https://doi.org/10.1016/00381101(68)90020-8

[122] H. Kleemann, B. Lüssem, K. Leo, J. Appl. Phys. 111 (2012), 123722. https://doi.org/10.1063/1.4730771

[123] W. B. Berry, Appl. Phys. Lett. 25 (1974), 195. https://doi.org/10.1063/1.1655436

[124] A. R. Moore, Appl. Phys. Lett. 27 (1975), 26. https://doi.org/10.1063/1.88275

[125] A. R. Moore, RCA Review 36 (1975), 551.

[126] O. Almora, G. Garcia-Belmonte, Solar Energy 189 (2019), 103. https://doi.org/10.1016/j.solener.2019.07.048

[127] R. S. Crandall, Appl. Phys. Lett. 42 (1983), 451. https://doi.org/10.1063/1.93967

[128] C. C. McAndrew, B. K. Bhattacharyya, O. Wing, IEEE Trans. Comput.-Aided Des. Integr. Circuits Syst. 12 (1993), 825. https://doi.org/10.1109/43.229757

[129] P. V. Halen, "A New Semiconductor Junction Diode Space Charge Layer Capacitance Model", presented at Proceedings of the 1988 Bipolar Circuits and Technology Meeting, Minneapolis, USA, 1988. https://doi.org/10.1109/BIPOL.1988.51070

[130] J. E. Parrott, Int. J. Electron. 47 (1979), 561. https://doi.org/10.1080/00207217908938679

[131] S. K. Sharma, D. Pavithra, G. Sivakumar, N. Srinivasamurthy, B. L. Agrawal, Sol. Energy Mater. Sol. Cells 26 (1992), 169. https://doi.org/10.1016/0927-0248(92)90058-W

[132] J. Bisquert, Phys. Chem. Chem. Phys. 5 (2003), 5360. https://doi.org/10.1039/B310907K

[133] I. Mora-Seró et al., Sol. Energy Mater. Sol. Cells 92 (2008), 505. https://doi.org/10.1016/j.solmat.2007.11.005

[134] O. Almora, L. G. Gerling, C. Voz, R. Alcubilla, J. Puigdollers, G. Garcia-Belmonte, Sol. Energy Mater. Sol. Cells 168 (2017), 221. https://doi.org/10.1016/j.solmat.2017.04.042

[135] X. Xu et al., ACS Nano 9 (2015), 1782. https://doi.org/10.1021/nn506651m

[136] F. Zhou et al., ACS Nano 10 (2016), 5900. https://doi.org/10.1021/acsnano.6b01202

[137] P. Du et al., Adv. Funct. Mater. 25 (2015), 2420. https://doi.org/10.1002/adfm.201500335

[138] J. Xu, Z. Ku, Y. Zhang, D. Chao, H. J. Fan, Adv. Mater. Technol. 1 (2016), 1600074. https://doi.org/10.1002/admt.201600074

[139] Z. Liu et al., ACS Appl. Mater. Interfaces 9 (2017), 22361. https://doi.org/10.1021/acsami.7b01471

[140] J. Liang et al., J. Mater. Chem. A 6 (2018), 2047. https://doi.org/10.1039/C7TA09099D

[141] J. Liang et al., Nano Energy 52 (2018), 239. https://doi.org/10.1016/j.nanoen.2018.07.060

[142] S. Intermite et al., Electrochim. Acta 258 (2017), 825.

https://doi.org/10.1016/j.electacta.2017.11.132

[143] C. H. Ng et al., ACS Appl. Energy Mater. 1 (2018), 692. https://doi.org/10.1021/acsaem.7b00103

[144] A. Slonopas, H. Ryan, P. Norris, Electrochimica Acta 307 (2019), 334.

https://doi.org/10.1016/j.electacta.2019.03.221 


\section{Chapter 3. Characterization techniques: concepts and instrumentation}

In the previous chapter, the theoretical formalisms regarding the working principles of solar cells, their current-voltage $(J-V)$ characteristic and their capacitance $(C)$ were introduced. These are vital concepts for understanding photovoltaic devices, and thus perovskite solar cells (PSCs). Complementary, in this chapter we focus in the experimental instrumentation and procedures for characterizing the electrical response of the studied samples in direct and alternating current modes, DC and AC respectively. Specially, impedance spectroscopy (IS) is introduced.

\subsection{Structure and materials of the characterized samples}

The main focus for the characterizations presented in the subsequent chapters was set on PSCs with regular structure and $\mathrm{CH}_{3} \mathrm{NH}_{3} \mathrm{PbI}_{3}$ (MAPI) as absorber material. ${ }^{[1-13]}$ The most typical regular structure (see Section 2.2), where $\mathrm{TiO}_{2}$ and spiro-OMeTAD serve as electron and hole selective contacts, ETM and HTM respectively, was systematically studied under several material engineering variations. $\left.{ }^{[1-2,}, 4,6-12\right]$ Examples of these were the morphology (compact or mesoporous) of the $\mathrm{TiO}_{2}$ layer(s) and the inclusion of capping intermediate layers. Alternatively, the inverted structure was studied. ${ }^{[7,10]}$ Material samples (not devices) were also considered in some experiments. For instance: MAPI pellets ${ }^{[5]}$ and sandwiched contacted sequence of layers. ${ }^{[7]}$ Furthermore, some experiments were made on devices with regular structure based on mixed cation/anion perovskites, incorporating cesium, formamidinium and bromide. ${ }^{[1-12,14]}$

Every sample structure and/or material characterized by the author and considered in this work is listed in Table 3.1. There, the labeling used in the following chapters is also specified, as well as the corresponding published article reference. In most of the cases these publications will be considered in deep during the following chapters. Otherwise they will be lightly mentioned.

Note that the only devices fabricated by the author were those identified as mTmapiClS in Table 3.1. In the rest of the cases the samples were provided, and in all cases the description of the corresponding fabrication procedures can be found in the references. The focus of this work is set on the characterization of the electrical behavior of PSCs, not their fabrication. Nevertheless, a brief section is included subsequently illustrating the main concepts of the general solutionprocessed production of PSCs.

Table 3.1. Different labeling and structures of the studied samples.

\begin{tabular}{ccc}
\hline Label & Structure & Ref. \\
\hline mTmapiClS & $\mathrm{FTO} / \mathrm{c}-\mathrm{TiO}_{2} / \mathrm{m}-\mathrm{TiO}_{2} / \mathrm{CH}_{3} \mathrm{NH}_{3} \mathrm{PbI}_{3-\mathrm{x}} \mathrm{Cl}_{x} /$ spiroOMeTAD$/ \mathrm{Au}$ & {$[1-4]$} \\
cTmapiClS & $\mathrm{FTO} / \mathrm{c}-\mathrm{TiO}_{2} / \mathrm{CH}_{3} \mathrm{NH}_{3} \mathrm{PbI}_{3-\mathrm{x}} \mathrm{Cl}_{x} / \mathrm{spiroOMeTAD} / \mathrm{Au}$ & {$[1]$} \\
mTmapiS1 & $\mathrm{FTO} / \mathrm{c}-\mathrm{TiO}_{2} / \mathrm{m}-\mathrm{TiO}_{2} / \mathrm{CH}_{3} \mathrm{NH}_{3} \mathrm{PbI}_{3} / \mathrm{spiroOMeTAD} / \mathrm{Au}$ & {$[4]$} \\
cTmapiS1 & $\mathrm{FTO} / \mathrm{c}-\mathrm{TiO}_{2} / \mathrm{CH}_{3} \mathrm{NH}_{3} \mathrm{PbI}_{3} / \mathrm{spiroOMeTAD} / \mathrm{Au}$ & \\
MapiPellet & $\mathrm{CH}_{3} \mathrm{NH}_{3} \mathrm{PbI}_{3}$ & {$[5]$} \\
mTmapiS2 & $\mathrm{FTO} / \mathrm{c}-\mathrm{TiO}_{2} / \mathrm{m}_{-}-\mathrm{TiO}_{2} / \mathrm{CH}_{3} \mathrm{NH}_{3} \mathrm{~Pb} \mathrm{~Pb}_{3} / \mathrm{spiroOMeTAD} / \mathrm{Au}$ & {$[7]$} \\
cTmapiS2 & $\mathrm{FTO} / \mathrm{c}-\mathrm{TiO}_{2} / \mathrm{CH}_{3} \mathrm{NH}_{3} \mathrm{PbI}_{3} / \mathrm{spiroOMeTAD} / \mathrm{Au}$ &
\end{tabular}




\begin{tabular}{|c|c|c|}
\hline Label & Structure & Ref. \\
\hline InvMapi & ITO/PEDOT:PSS/ $\mathrm{CH}_{3} \mathrm{NH}_{3} \mathrm{PbI}_{3} / \mathrm{PC}_{70} \mathrm{BM} / \mathrm{Ag}$ & {$[7,10]$} \\
\hline FmapiClS & $\begin{array}{l}\mathrm{FTO} / \mathrm{c}-\mathrm{TiO}_{2} / \mathrm{m}-\mathrm{TiO}_{2} / \mathrm{SAM} / \mathrm{CH}_{3} \mathrm{NH}_{3} \mathrm{PbI}_{3-\mathrm{x}} \mathrm{Cl}_{\mathrm{x}} / \\
\text { spiroOMeTAD} / \mathrm{Au}\end{array}$ & [8] \\
\hline mTmapiS3 & $\mathrm{FTO} / \mathrm{c}-\mathrm{TiO}_{2} / \mathrm{m}-\mathrm{TiO}_{2} / \mathrm{CH}_{3} \mathrm{NH}_{3} \mathrm{PbI}_{3} /$ spiroOMeTAD$/ \mathrm{Au}$ & [9] \\
\hline 3Dmapi & $\mathrm{FTO} / \mathrm{c}-\mathrm{TiO}_{2} / \mathrm{m}-\mathrm{TiO}_{2} / \mathrm{CH}_{3} \mathrm{NH}_{3} \mathrm{PbI}_{3} / \mathrm{spiroOMeTAD} / \mathrm{Au}$ & {$[11-$} \\
\hline 2DTiOmapi & $\begin{array}{c}\mathrm{FTO} / \mathrm{c}-\mathrm{TiO}_{2} / \mathrm{m}-\mathrm{TiO}_{2} / \mathrm{AVA}_{2} \mathrm{PbI}_{4} / \mathrm{CH}_{3} \mathrm{NH}_{3} \mathrm{PbI}_{3} / \\
\text { spiroOMeTAD/Au }\end{array}$ & $\begin{array}{l}12, \\
15]\end{array}$ \\
\hline 2DspiroMapi & $\mathrm{FTO} / \mathrm{c}-\mathrm{TiO}_{2} / \mathrm{m}_{-}-\mathrm{TiO}_{2} / \mathrm{CH}_{3} \mathrm{NH}_{3} \mathrm{PbI}_{3} / \mathrm{Bl}_{2} \mathrm{PbI}_{4} /$ spiroOMeTAD $/ \mathrm{Au}$ & \\
\hline 2D3D2Dmapi & $\begin{array}{c}\mathrm{FTO} / \mathrm{c}-\mathrm{TiO}_{2} / \mathrm{m}-\mathrm{TiO}_{2} / \mathrm{AVA}_{2} \mathrm{PbI}_{4} / \mathrm{CH}_{3} \mathrm{NH}_{3} \mathrm{PbI}_{3} / \mathrm{Bl}_{2} \mathrm{PbI}_{4} / \\
\text { spiroOMeTAD/Au }\end{array}$ & \\
\hline 3Dmix & $\begin{array}{c}\mathrm{FTO} / \mathrm{c}-\mathrm{TiO}_{2} / \mathrm{m}_{-} \mathrm{TiO}_{2} / \mathrm{Cs}_{0.1} \mathrm{FAA}_{0.74 \mathrm{MA}}{ }_{0.13} \mathrm{PbI}_{2.48} \mathrm{Br}_{0.39} / \\
\text { spiroOMeTAD/Au }\end{array}$ & \\
\hline 2DspiroMix & $\begin{array}{c}\mathrm{FTO} / \mathrm{c}-\mathrm{TiO}_{2} / \mathrm{m}_{-}-\mathrm{TiO}_{2} / \mathrm{Cs}_{0.1} \mathrm{FA}_{0.74} \mathrm{MA}_{0.13} \mathrm{PbI}_{2.48} \mathrm{Br}_{0.39} / \\
\mathrm{PEA}_{2} \mathrm{PnI}_{4} / \mathrm{spiroOMeTAD} / \mathrm{Au}\end{array}$ & \\
\hline 2D3D2Dmix & $\begin{array}{c}\mathrm{FTO} / \mathrm{cTiO}_{2} / \mathrm{mTiO}_{2} / \mathrm{PEA}_{2} \mathrm{PnI}_{4} / \mathrm{Cs}_{0.1} \mathrm{FA}_{0.74} \mathrm{MA}_{0.13} \mathrm{PbI}_{2.48} \mathrm{Br}_{0.39} / \\
\mathrm{PEA}_{2} \mathrm{PnI}_{4} / \mathrm{spiroOMeTAD}_{\mathrm{suu}}\end{array}$ & \\
\hline Smapi1 & $\mathrm{ITO} / \mathrm{C}_{60}-\mathrm{SAM} / \mathrm{CH}_{3} \mathrm{NH}_{3} \mathrm{PbI}_{3} / \mathrm{P} 3 \mathrm{HT} / \mathrm{Ta}-\mathrm{WO}_{x} / \mathrm{Au}$ & [16] \\
\hline Smapi2 & $\mathrm{ITO} / \mathrm{C}_{60}-\mathrm{SAM} / \mathrm{CH}_{3} \mathrm{NH}_{3} \mathrm{PbI}_{3} / \mathrm{PDCBT} / \mathrm{Ta}-\mathrm{WO}_{\mathbf{x}} / \mathrm{Au}$ & \\
\hline PmixP & $\begin{array}{c}\mathrm{ITO} / \mathrm{m}-\mathrm{SnO}_{2} / \mathrm{PC}_{61} \mathrm{BM} / \mathrm{FA}_{0.83} \mathrm{MA}_{0.17} \mathrm{~Pb}_{1.1} \mathrm{Br}_{0.22} \mathrm{I}_{2.98} / \\
\mathrm{PDCBT} / \mathrm{Ta}-\mathrm{WO}_{\mathrm{x}} / \mathrm{Au}\end{array}$ & [12] \\
\hline PPmixP & $\begin{array}{c}\mathrm{ITO} / \mathrm{SnO}_{2} / \mathrm{PMMA}(\mathrm{PCBM}) / \mathrm{Cs}_{0.05} \mathrm{MA}_{0.1} \mathrm{FA}_{0.85} \mathrm{~Pb}\left(\mathrm{I}_{0.85} \mathrm{Br}_{0.15}\right)_{3} / \\
\text { PDCBT/Ta-WO } / \mathrm{Au}\end{array}$ & [14] \\
\hline SmixP & $\begin{array}{c}\mathrm{ITO} / \mathrm{SnO}_{2} / \mathrm{Cs}_{0.05} \mathrm{MAA}_{0.1} \mathrm{FA}_{0.85} \mathrm{~Pb}\left(\mathrm{I}_{0.85} \mathrm{Br}_{0.15}\right)_{3} / \\
\text { PDCBT/Ta-WO } / \mathrm{Au}\end{array}$ & \\
\hline SmixIP & $\mathrm{ITO} / \mathrm{SnO}_{2} / \mathrm{Cs}_{0.15} \mathrm{FA}_{0.85} \mathrm{PbI}_{3} / \mathrm{PDCBT} / \mathrm{Ta}-\mathrm{WO}_{\mathrm{x}} / \mathrm{Au}$ & \\
\hline
\end{tabular}

\subsubsection{Fabrication of perovskite solar cells}

The generic procedure for the fabrication of a typical perovskite solar cells with regular structure is depicted in Figure 3.1. Note that most of the steps are solution-process based and the annealing periods consist on a few hours at temperatures around $500^{\circ} \mathrm{C}$, at most. The details on the solutions, annealing times, spinning speeds, among others, can be found in the references of Table 3.1. More general reviews can be found in the literature. ${ }^{[17-18]}$ Here, a brief description is commented (see also Section 2.2).

First, the glass/TCO substrate is prepared for the deposition of the next layer. This includes the patterning of the contact design (e.g. laser or solution etching, see Figure 2.3) and the cleaning. The latter typically includes soap washing, solution sonication and/or thermal annealing under ultraviolet (UV) radiation with ozone atmosphere.

The deposition of the selective contacts obviously depends on the structure and materials. Most frequently, the ETM with compact and mesoporous $\mathrm{TiO}_{2}$ layers is made by the sequenced spray pyrolysis and spin coating, respectively. After every spin coating there typically is an annealing step, where the material crystallization is thermally regulated. $\mathrm{The}^{\mathrm{TiO}}{ }_{2}$ layers are 
nearly transparent, with a light yellow tone, as shown in the pictures of upper panel of Figure 3.1. This is an expected behavior since the window material may transmit most of the light (high bandgap) to the absorber material.

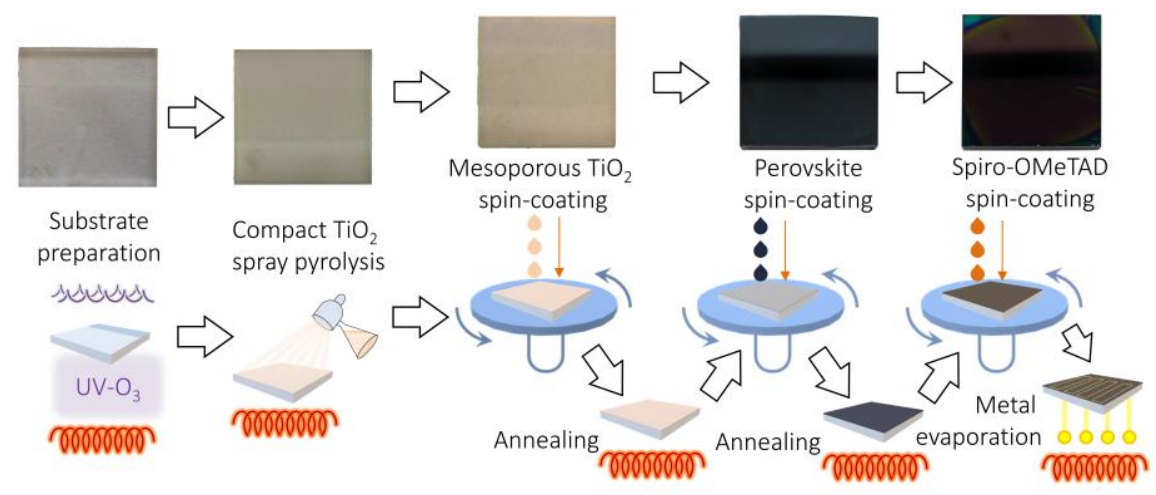

Figure 3.1. Schemed generic fabrication process of perovskite solar cells, from left to right.

Pictures in the upper panel courtesy of M.G-B.

The deposition of the perovskite is most typically made by spin coating. Then, there are subsequent thermal annealing of the perovskite layer, later the HTM (e.g. spiro-OMeTAD) is deposited by spin coating and lastly the metal electrode is evaporated. As the crystallization of the perovskite occurs, the intense dark color arises indicating the strong absorption of the material. Furthermore, the border effects are more evident in the picture after the spiro spin-coating. This indicate how the spinning of the solution may create some minor morphology differences between different pixels in the same substrate.

\subsection{Dynamic current-voltage characteristic}

The $J-V$ curve in PSCs presents a remarkable hysteresis-like behavior, depending on the measurement ways, as introduced in Section 2.4. Experimentally, the first consequence here is that the power conversion efficiency (PCE) cannot be taken straightforwardly from a single $J$ - $V$ under standard illumination intensity. Also note, that the dynamic evolution of the several $J-V$ curve patterns are of interest, as discussed in subsequent chapters. These two reasons needed an evolution of the setups traditionally used to measure the PCE and the study of the dynamic $J-V$ curves in PSCs.

For evaluation the PCE in PSCs the maximum power point (mpp) tracking is the most advisable procedure. It consist in perform one initial $J-V$ curve between $\mathrm{SC}$ and OC, set the sample at the corresponding voltage $V_{m p p}$ for a time $t_{m p p t}$, afterwards it is measured the current in a voltage section around the previous $V_{m p p}$. If steady $V_{m p p}$, the previous $V_{m p p}$ is maintained another $t_{m p p t}$, if not, the new $V_{m p p}$ value is set for the next $t_{m p p t}$. Purposely, the works by Pellet et al. ${ }^{[19]}$ and Rakocevic et al. ${ }^{[20]}$ are very illustrative of different algorithms and the measurement details. 
For the study of the dynamic $J-V$ curves, and hence the hysteresis, in PSCs it is needed to use multimeter or potentiostat workstations with a software interface including a "measurement sequence builder". The latter implies that the software may be able to set continuous routines including steady polarization periods and dynamic voltage sweeping periods, with or without DC illumination. During the voltage sweeping periods, the control of the voltage scan rate is of particular importance for the experiments discussed in subsequent chapters.

Importantly, degradation issues can be significant while measuring the electrical response from PSCs, not only DC but in general. Specifically, moisture can undermine the reliability and reproducibility of the measurement results. In this regard, it is recommended to perform the experiments in $\mathrm{N}_{2}$ atmosphere. For some applications, this can be done by introducing the complete setup inside a glove box. Alternatively, and possibly more practically, the samples are placed in holders with $\mathrm{N}_{2}$ atmosphere and glass windows.

\subsection{AC spectroscopic techniques}

In the previous sections the context was provided on what samples were studied and what should be considered for measuring the traditional $J-V$ characteristic. Here, the framework of the impedance spectroscopy (IS) is introduced. The potentiostat setup is also commented, from which some IS variants and light modulated spectroscopies are available.

\subsubsection{Potentiostatic impedance spectroscopy}

The impedance was introduced between 1880 and 1900 by Heaviside as a complex transfer function which expresses the ratio between complex voltage and current. ${ }^{[21]}$ In the potentiostatic variant, a small voltage perturbation is applied and the electrical current response is measured, defining the impedance $Z$ with units of Ohms. ${ }^{[22]}$ The IS characterization technique is an stablished method for the assessment of the resistive, capacitive and inductive properties of materials and complete devices. In the case of photovoltaic cells, the IS can be used to elucidate the main recombination mechanisms, ${ }^{[11]}$ the doping densities, ${ }^{[4,23]}$ deep defect levels ${ }^{[2]}$ and the density of states. ${ }^{[24]}$

Formally, one can consider a sample at DC voltage $\bar{V}$ where a steady-state current density $\bar{J}(\bar{V})$ is measured. Then, a small applied voltage perturbation $\tilde{V}(t)=|\tilde{V}| \exp [i \omega t]$ with angular frequency $\omega$ can be applied in AC mode, as illustrated in Figure 3.2a. Here $i=\sqrt{-1}$ is the imaginary unit. Thus, the total applied voltage results

$$
V=\bar{V}+|\tilde{V}| \exp [i \omega t]
$$

and the corresponding current might respond as

$$
J=\bar{J}+\tilde{J} \exp [i \omega t]
$$

Here the phasor-related part $\tilde{J}$ carriers the information on the differential resistance and capacitances for the sample. Hence, a sinusoidal $\tilde{V}(t)$ like Figure 3.2a originates a $\phi$ phase shifted current response, as in Figure $3.2 \mathrm{~b}$, which can be written as $\tilde{J}=|\tilde{J}| \exp [-i \phi]$. Therefore, the impedance is defined as 
$Z(\omega)=\frac{\widetilde{V}(t)}{\tilde{J}(t)}=\frac{|\widetilde{V}|}{|\tilde{J}|} \exp [i \phi]=|Z| \exp [i \phi]$

The $\phi$-dependence on frequency $f=\omega / 2 \pi$ generates an impedance spectrum. Most commonly, the impedance spectra are represented as $Z(\omega)=Z^{\prime}(\omega)+i Z^{\prime \prime}(\omega)$. The latter is the Nyquist plot representation, as shown in Figure 3.2c, where a distinctive semicircle is depicted from a linear resistor-capacitor $(R C)$ couple with a single response time constant $\tau$.

(a)

(b)

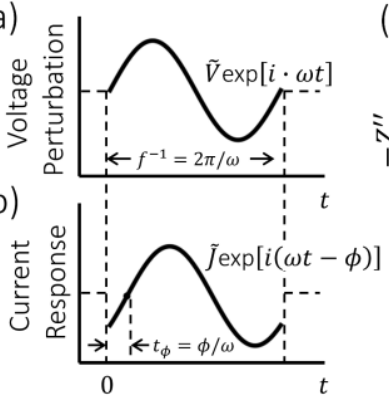

(c)
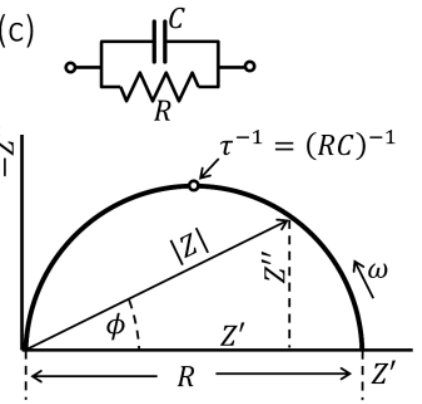

(d)

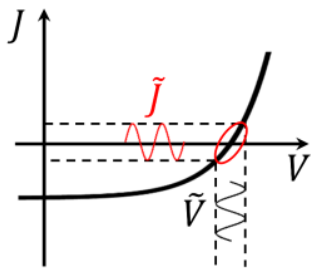

Figure 3.2. Impedance spectroscopy (a) small voltage perturbation, (b) current signal, (c) impedance Nyquist plot for a CR couple and (d) scheme of measurement around DC open circuit in an illuminated solar cell.

The real part of the impedance $Z^{\prime}$ informs on the energy dissipation, which is expressed as differential resistance. At the lowest frequencies $(\omega \rightarrow 0)$ there is nearly no phase shift $(\phi \rightarrow 0)$, thus $Z \rightarrow Z^{\prime}$ making the total differential resistance as the radius of the semicircle in the Nyquist plot. In contrast, the imaginary part of impedance $Z^{\prime \prime}$ carries the information on the energy storage, which is expressed as the differential capacitance. The maximum absolute value of $-Z^{\prime \prime}$ ( $\phi=\pi / 4$ in Figure 3.2c) corresponds to the characteristic angular frequency $\omega_{\tau}=\tau^{-1}=$ $(R \cdot C)^{-1}$ of the $R C$ couple.

Importantly, definition (3.3) is only valid under the linearity approximation. For instance, in ohmic samples the current behaves linear with voltage and the "size" of the perturbation is not an issue. However, in non-ohmic samples where the resistance depends on the voltage, the perturbation $\widetilde{V}$ should be small enough so the region of the sampled DC $J-V$ curve can be approached to a linear one. This is illustrated in Figure 3.2d for a typical solar cell under illumination, where the perturbation was applied around open circuit. In practice, it is recommendable to use potentiostatic workstations whose software includes numerical algorithms testing linearity at each measured frequency. An example of this is the significance parameter implemented in the Zahner setup and introduced by Schiller and Kaus ${ }^{[25]}$.

By measuring the IS, one is simultaneously accessing to variate magnitudes. Here we focus in capacitance, inductance and AC conductivity, besides impedance. Note that that AC conductivity is a volume-normalized form of the admittance $Y$. In Table 3.2 their different complex definitions are listed, as well as the relations among them. Also in Table 3.2, there are the expressions for the real and imaginary parts of these magnitudes as a function of typical 
measurement data: $Z^{\prime}$ and $Z^{\prime \prime}$ or $|Z|$ and $\phi$. This is a handy list for the ulterior parametric representations, e.g. Nyquist $Z^{\prime \prime}\left(Z^{\prime}\right)$ or Bode $Z^{\prime}(\omega)$ and $Z^{\prime \prime}(\omega)$ plots.

The real parts of $Z, C^{*}, L^{*}$ and $\sigma^{*}$ towards the limit $\omega \rightarrow 0$ indicate total resistance, capacitance, inductance and DC conductivity, respectively. The real part of impedance $Z^{\prime}$ indicates the energy dissipation through resistive mechanisms. The imaginary part $Z^{\prime \prime}$ is the reactance, which relates with the energy storage. For instance, the capacitive and inductive reactances typically indicate energy storage in form of electric and magnetic fields, respectively. If only dielectric relaxation processes are considered, from the magnitudes in Table 3.2 we can obtain the complex dielectric constant $\varepsilon^{*}=d C^{*}$ and the complex electric modulus $M^{*}=1 / \varepsilon^{*}=$ $L^{*} / d$, where $d$ is the distance between the electrodes. ${ }^{[22,26]}$

The AC conductivity is a normalized form of the admittance $Y^{*}=1 / Z$. They are related as $Y^{*}=\sigma^{*} / d$. From the latter relation, the conductance and susceptance can be expressed as $Y^{\prime}=$ $\sigma^{\prime} / d$ and $Y^{\prime \prime}=\sigma^{\prime \prime} / d$, respectively. This normalization makes the conductivity a unique property of the sample, regardless of the geometry. Note that the admittance carries the exact information as the impedance. This is the reason why some methods/ procedures are indistinctly named impedance or admittance spectroscopies in different contexts. An example of this is the thermal admittance spectroscopy (TAS), as subsequently commented in Section 3.3.3.

The different spectroscopic representations from magnitudes in Table 3.2 carry an specific information which can be accessed by the suitable model. The spectra are typically fitted by numerical methods to these models and the studied sample is characterized in terms of the fitting parameters. There are two main approaches here: the solution of the time dependent transport equations (see Section 2.3) or the equivalent circuit (EC) models.

Table 3.2. Definitions and expressions for real and imaginary parts of impedance, capacitance, inductance and DC conductivity as a function of frequency. Here, $d$ is the distance between electrodes.

\begin{tabular}{|c|c|c|c|c|}
\hline $\begin{array}{l}\text { Definition as a } \\
\text { function of }(\ldots)\end{array}$ & $\begin{array}{l}\text { Impedance } \\
\left(\Omega \mathrm{cm}^{2}\right)\end{array}$ & $\begin{array}{l}\text { Capacitance } \\
\left(\mathrm{F} \mathrm{cm}^{-2}\right)\end{array}$ & $\begin{array}{l}\text { Inductance } \\
\left(\mathrm{H} \mathrm{cm}^{2}\right)\end{array}$ & $\begin{array}{l}\text { AC conductivity } \\
\left(\mathrm{S} \mathrm{cm}^{-1}\right)\end{array}$ \\
\hline Complex (Z) & $Z$ & $C^{*}=\frac{1}{i(1) 7}$ & $L^{*}=\frac{Z}{i(\omega}$ & $\sigma^{*}=\frac{d}{7}$ \\
\hline Complex $\left(\mathrm{C}^{*}\right)$ & $Z=\frac{1}{i \omega C^{*}}$ & $C^{*}$ & $L^{*}=-\frac{1}{\omega^{2} C^{*}}$ & $\sigma^{*}=i \omega d C^{*}$ \\
\hline Complex $\left(L^{*}\right)$ & $Z=i \omega L^{*}$ & $C^{*}=-\frac{1}{\omega^{2} L^{*}}$ & $L^{*}$ & $\sigma^{*}=\frac{d}{i \omega L^{*}}$ \\
\hline Complex $\left(\sigma^{*}\right)$ & $Z=\frac{d}{\sigma^{*}}$ & $C^{*}=\frac{\sigma^{*}}{i \omega d}$ & $L^{*}=\frac{d}{i \omega \sigma^{*}}$ & $\sigma^{*}$ \\
\hline $\operatorname{Re}\left(Z^{\prime}, Z^{\prime \prime}\right)$ & $Z^{\prime}$ & $C^{\prime}=\frac{-Z^{\prime \prime}}{\omega\left(Z^{\prime 2}+Z^{\prime \prime 2}\right)}$ & $L^{\prime}=\frac{Z^{\prime \prime}}{\omega}$ & $\sigma^{\prime}=\frac{Z^{\prime} d}{\left(Z^{\prime 2}+Z^{\prime \prime 2}\right)}$ \\
\hline $\operatorname{Im}\left(Z^{\prime}, Z^{\prime \prime}\right)$ & $Z^{\prime \prime}$ & $C^{\prime \prime}=\frac{-Z^{\prime}}{\omega\left(Z^{\prime 2}+Z^{\prime 2}\right)}$ & $L^{\prime \prime}=\frac{-Z^{\prime}}{\omega}$ & $\sigma^{\prime \prime}=\frac{-Z^{\prime \prime} d}{\left(Z^{\prime 2}+Z^{\prime \prime 2}\right)}$ \\
\hline $\operatorname{Re}(|Z|, \phi)$ & $Z^{\prime}=|Z| \cos [\phi]$ & $C^{\prime}=\frac{-\sin [\phi]}{\omega|Z|}$ & $L^{\prime}=\frac{|Z| \sin [\phi]}{\omega}$ & $\sigma^{\prime}=\frac{d \cos [\phi]}{|Z|}$ \\
\hline $\operatorname{Im}(|Z|, \phi)$ & $Z^{\prime \prime}=|Z| \sin [\phi]$ & $C^{\prime \prime}=\frac{-\cos [\phi]}{\omega|Z|}$ & $L^{\prime \prime}=\frac{-|Z| \cos [\phi]}{\omega}$ & $\sigma^{\prime \prime}=\frac{-d \sin [\phi]}{|Z|}$ \\
\hline
\end{tabular}

The time dependent solution of the drift-diffusion equations (DDE) in photovoltaic solar cells depend on more fundamental and variated parameters. Typically, one would need the mobilities 
$\left(\mu_{n}, \mu_{p}\right)$, diffusion coefficients $\left(D_{n}, D_{p}\right)$, recombination coefficients $\left(\tau_{n}, \tau_{p}, \beta, A_{n}, A_{p}\right)$, recombination velocity $\left(S_{r n}, S_{r p}\right)$, intrinsic concentration $\left(n_{i}\right)$, doping concentrations $\left(N_{A}, N_{D}\right)$, dielectric constant $(\varepsilon)$ and built-in voltage $\left(V_{b i}\right)$. Moreover, in the case of heterostructures some of these parameters should be defined at each section between the electrodes. This is of course, a very detailed description, at the cost of a higher time and machine consuming task.

In the EC model method, the system is approached to an array of simpler circuit elements whose behavior simulate the experimental spectra. Note that, with the appropriate assumptions, EC and DDE methods may be equivalent, and the parameters in one should be possible to be expressed as a function of the other. The circuit elements which will be used in subsequent chapters are summarized in Table 3.3, i.e resistors, capacitors, inductors and constant phase elements (CPE). There, a summary on units, scheme, impedance, capacitance, phase and the time constant $\tau$ assuming a coupled resistor $R$ are also provided.

Interestingly, inductors can create negative capacitance-like behavior, despite their storage energy mechanism is fundamentally different. Furthermore, the CPE approaches a capacitor or a Warburg diffusion element when its dimensionless non-linearity power parameter $\theta \rightarrow 1$ or $\theta \rightarrow$ $1 / 2$, respectively. In the case $\theta=1$, the CPE coefficient parameter $\Theta$ is $\mathrm{C}$, otherwise the units of $\Theta$ also depend on $\theta$.

Table 3.3. Circuit elements; their representation and expressions. The star $\star$ signals a coupling with a resistor $R$.

\begin{tabular}{|c|c|c|c|c|c|c|c|}
\hline Circuit element & $\begin{array}{c}\text { Paramet } \\
\text { er }\end{array}$ & Unit & Scheme & $\begin{array}{c}\text { Impedan } \\
\text { ce }\end{array}$ & Capacitance & $\phi$ & $\star \tau$ \\
\hline Resistor & $R$ & $\Omega \mathrm{cm}^{2}$ & $\sqrt{ }$ & $R$ & - & $0^{\circ}$ & - \\
\hline Capacitor & C & $\mathrm{F} \mathrm{cm}^{-2}$ & & $\frac{1}{i \omega C}$ & C & $-90^{\circ}$ & $R C$ \\
\hline Inductor & $L$ & $\mathrm{H} \mathrm{cm}^{2}$ & 902 & $i \omega L$ & $-\frac{1}{\omega^{2} L}$ & $90^{\circ}$ & $\frac{R}{L}$ \\
\hline $\begin{array}{l}\text { Constant phase } \\
\text { element (CPE) }\end{array}$ & $\begin{array}{l}\Theta \\
\theta\end{array}$ & $\begin{array}{c}\mathrm{F} \mathrm{cm}^{-2} \\
\mathrm{~s}^{\theta-1} \\
-\end{array}$ & 11 & $\frac{1}{\Theta(i \omega)^{\theta}}$ & $\star \frac{(R \Theta)^{\frac{1}{\theta}}}{R}$ & $-\theta \cdot 90^{\circ}$ & $(R \Theta)^{\frac{1}{\theta}}$ \\
\hline
\end{tabular}

Importantly, Table 3.3 lists frequency independent parameters. They share units and causality relations with some magnitudes in Table 3.2, but they should not be mistaken. Each array of circuit elements forms an EC with an unique theoretical IS spectra. But each experimental IS spectra with $N_{f}$ points/frequencies can be modeled by a combinatorial number of EC with up to $N_{f}$ circuit element parameters each. Fitting accuracy increases as $N_{f}$, thus, in practice, one measures spectra with as many points/frequencies as possible, at the cost of the time-consuming task as frequencies lessen. However, one also wants to give uniqueness and physical meaning to the used EC model. Therefore, one chooses the lower possible number of meaningful parameters, with an acceptable accuracy. "Physically meaningful EC models with just acceptable fittings are preferred over perfect fittings with parameters with ambiguous physical meanings". 
Each EC model has an overall total characteristic response time $\tau_{T}$, which could be integrated by individual time constants $\tau$, corresponding to individual sections of the EC. Last column of Table 3.3 shows the time constants for typical RC, RL of RCPE coupled circuit elements. For instance, in the Nyquist representation one single $\tau=R C$ couple would deliver an arc like that of Figure 3.2c. Further time constants would deform that arc (if similar $\tau$ ) or add extra arcs (if different $\tau$ ).

Detailed tutorials on equivalent circuits, their representations and IS analysis in solar cells can be found in the literature. ${ }^{[22,26-31]}$ Nevertheless, whatever the EC model is used, it may include resistive elements related with the transport mechanisms in Section 2.3 and capacitive elements as those described in Section 2.6. Furthermore, ohmic series resistance and inductance are also usually present, most typically as artifacts of the wires and connections.

Using IS to study solar cells under incident DC illumination power $\bar{P}_{\text {in }}$ is obviously of special interest here. In principle, there is no restrictions and one can measure IS at any quasi-steady state defined by DC light and voltage. However, in practice, potentiostatic IS when significant DC current is flowing is typically affected by inductive artifacts.

DC open-circuit condition is the most typical IS characterization routine for studying solar cells under illumination. In this procedure a series of $\bar{P}_{\text {in }}$ values are set, and for each one the DC voltage corresponding to the $\bar{V}_{o c}$ is applied. This quasi-open-circuit state typically only let currents between nanoamps and microamps. Then the small AC voltage perturbation $\tilde{V} \sim 10 \mathrm{mV}$ is applied, and the current response is measured in the spectrum frequency range. Given that photocurrent charge extraction is canceled by injected recombination current, the complete measurement is mostly informing on recombination phenomena. The procedure is repeated for several light intensities, as schemed in Figure 3.3a.
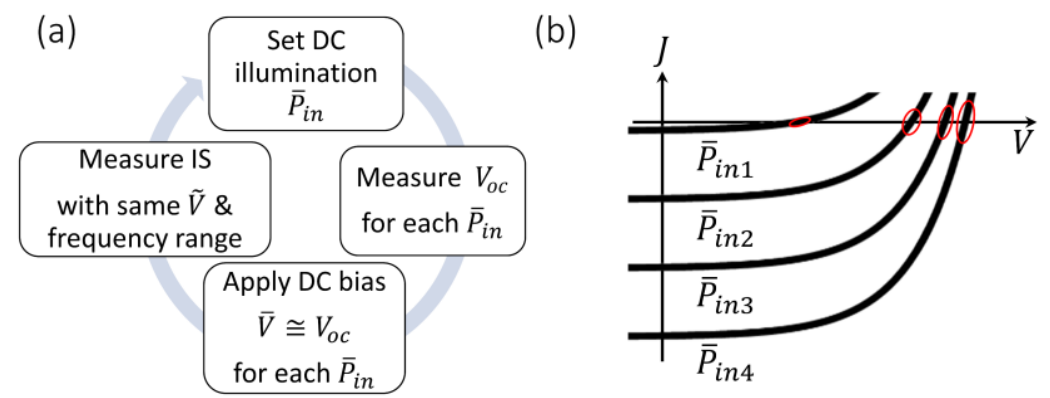

Figure 3.3. Measurements of impedance spectroscopy as a function of open-circuit voltage in solar cells under different illumination intensities: (a) schemed procedure and (b) rough approximation of the effect on the $J-V$ curve for incident light intensities $\bar{P}_{\text {in } 1}<\bar{P}_{i n 2}<$ $\bar{P}_{i n 3}<\bar{P}_{\text {in } 4}$. The red ellipses in (b) indicate the perturbation $\tilde{V}$ and corresponding current response $\tilde{J}$.

The IS measurements at quasi-OC mainly explore the recombination mechanisms in the voltage region around the values corresponding to mpp and $\mathrm{OC}$ for standard illumination intensity. For devices with large fill factor it can be difficult to measure $V_{o c}$ at low illumination intensities, like $\bar{P}_{i n 1}$ in Figure $3.3 \mathrm{~b}$, because the situation is not significantly different to dark, 
only that the illumination can generate noise artifacts. In PSCs, the hysteretic like mechanisms makes even harder the determination of the $V_{o c}$. Accordingly, larger illumination intensities are typically considered, like from $\bar{P}_{\text {in } 2}$ to $\bar{P}_{\text {in } 4}$ in Figure $3.3 \mathrm{~b}$.

The numerical simulations or fittings of IS spectra to EC models where performed in the ZView software from Scribner Associates. Further analytical simulations or fittings were made with Origin software from OriginLab and/or Mathematica, from Wolfram Research.

\subsubsection{Mott-Schottky measurement}

As introduced in Section 2.6.1 (see Table 2.1), the Mott-Schottky (MS) analysis aims to characterize the depletion layer capacitance $C_{d l}$ as a function of the DC voltage $\bar{V}$. Figure 3.4a shows a typical MS plot from two heterojunction silicon solar cells ${ }^{[23]}$ with different doping densities and built-in voltages. The measurement is mainly focused in reverse biases, where the depletion layer width expands and $C^{-2}(V)$ grows linearly. Higher slopes indicate lower doping and the intercept of the linear fittings with the voltage axis estimates $V_{b i}$.

Ideally, the MS procedure would start measuring the complete IS spectra at each DC bias. Then fitting the spectra to a complete EC model would provide, at each $\bar{V}$, the equivalent capacitance from the capacitor(s) coupled with the lowest time constant(s) (highest frequencies), i.e. from microseconds to milliseconds (from $\mathrm{kHz}$ to $\mathrm{MHz}$ ). But this would be a very timeconsuming task.

In practice, one single appropriate frequency $f_{d l}$ is sampled while varying the DC voltage, in the MS analysis. Then, the simplest RC circuits are assumed: series $C_{s}$ and $R_{s}$ and parallel $C_{p}$ and $R_{p}$, Figure 3.4b,c respectively. $C_{s}$ and $C_{p}$ are default MS analysis outcome from commercial measurement setups. The series circuit means an ideal capacitor $C_{S}$ with real series ohmic voltage drop through $R_{s}$. The parallel circuit implies a real capacitor $C_{s}$ with leakage currents through $R_{p}$, but ideally connected without ohmic series voltage drop. The simplest realistic scenario would be that of Figure 3.4d, where both series and parallel parasitic resistances are considered.
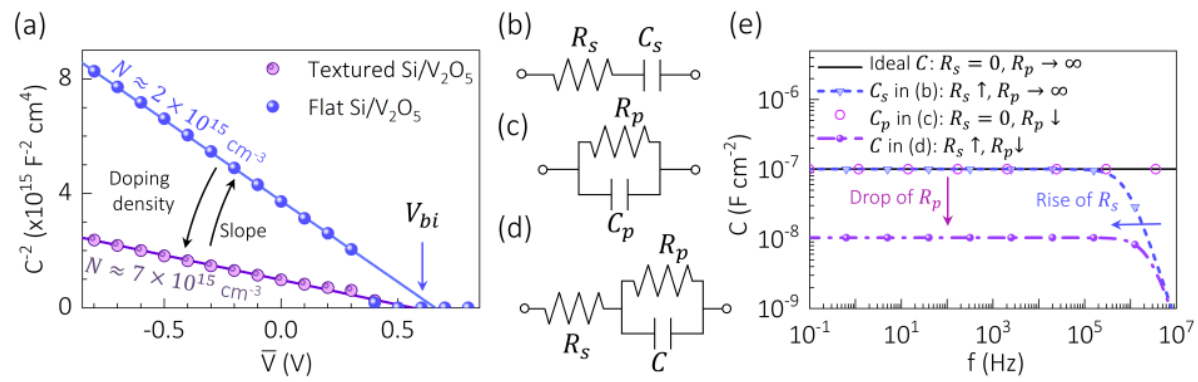

Figure 3.4. Mott-Schottky analysis. (a) MS plots from two silicon heterojunction solar cells. Data from ref. ${ }^{[23]}$. Copyright 2017, Elsevier B.V. Basic (b) parallel and (c) series equivalent circuits for the capacitance measurements.

The impedances and capacitances for the ECs in Figure 3.4b-d are summarized in Table 3.4. Note that $C^{\prime}$ represents the $C_{p}$ model. With the proper $R_{s} / R_{p}$ ratio, $C$ from Figure $3.4 \mathrm{~d}$ approaches 
better $C_{s}$ or $C_{p}$. Importantly, the selection of $f_{d l}$ must be within a plateau in the capacitance Bode plot representation, as in Figure 3.4e. The effects of increasing $R_{S}$ and decreasing $R_{p}$ are also illustrated there. Nevertheless, real samples may respond to EC similar or more complex than that of Figure 3.4d, without "perfect" plateaus at higher frequencies and/or significantly different $C_{s}$ and $C_{p}$. For instance, the discussions of Carr \& Chaudhary ${ }^{[32]}$ and Jarosz ${ }^{[33-34]}$ are illustrative in these regards.

Additional space charges due to contact barriers or irregularities in the doping profile may include extra circuit elements to the EC. This has been tackled by Guenther et al., ${ }^{[35]}$ who proposed a model where the knowledge of the exact equivalent circuit is not essentially needed.

In fully depleted devices, care must be taken when there is no clear linear behavior in the reverse biased regime. ${ }^{[2,4,10]}$ From numerical simulations by Kirchartz et al. ${ }^{[36]}$ it was suggested that the doping density extracted from the fitting of the section between equilibrium $0 \mathrm{~V}$ and $V_{b i}$ would be just an upper limit for the actual doping concentration. Also, Nigam et al. ${ }^{[37]}$ have discussed the electrode-related artifacts that could create MS-like behaviors in unintentionally doped organic thin film devices.

Table 3.4. Impedances and capacitances for the simplest RC equivalent circuits

\begin{tabular}{cccc}
\hline Parameter & Series $R_{s} C_{s}$ & Parallel $R_{p} C_{p}$ & Series $R_{s}$ parallel $R_{p} C$ \\
\hline Scheme & Figure 3.4b & Figure 3.4c & Figure 3.4d \\
$Z^{\prime}$ & $R_{S}$ & $\frac{R_{p}}{1+\omega^{2} R_{p}{ }^{2} C_{p}{ }^{2}}$ & $R_{s}+\frac{R_{p}}{1+\omega^{2} R_{p}{ }^{2} C^{2}}$ \\
$Z^{\prime \prime}$ & $-\frac{1}{\omega C_{s}}$ & $-\frac{\omega C_{p} R_{p}{ }^{2}}{1+\omega^{2} R_{p}{ }^{2} C_{p}{ }^{2}}$ & $-\frac{\omega R_{p}{ }^{2} C}{1+\omega^{2} R_{p}{ }^{2} C^{2}}$ \\
$|Z|^{2}$ & $R_{S}{ }^{2}\left(1+\frac{1}{\omega^{2} R_{s}{ }^{2} C_{s}{ }^{2}}\right)$ & $\frac{R_{p}{ }^{2}}{1+\omega^{2} R_{p}{ }^{2} C_{p}{ }^{2}}$ & $\frac{R_{p}{ }^{2}\left(\left(1+\frac{R_{s}}{R_{p}}\right)^{2}+\omega^{2} R_{s}{ }^{2} C^{2}\right)}{1+\omega^{2} R_{p}{ }^{2} C^{2}}$ \\
$C^{\prime}$ & $\frac{C_{s}}{1+\omega^{2} R_{s}{ }^{2} C_{s}{ }^{2}}$ & $C_{p}$ & $\frac{C}{\left(1+\frac{R_{s}}{R_{p}}\right)^{2}+\omega^{2} R_{s}{ }^{2} C^{2}}$ \\
$\begin{array}{c}\text { Practical } \\
\text { approach }\end{array}$ & $C_{s}=-\frac{1}{\omega Z^{\prime \prime}}$ & $C_{p}=-\frac{Z^{\prime \prime}}{\omega|Z|^{2}}$ & - \\
\hline
\end{tabular}

The estimation of $V_{b i}$ is always one of the most polemic in MS analyses. It is already evident from Table 2.1 that some effects may shift $V_{b i}$, for instance $2 k_{B} T$ due to majority carriers or $q N w_{i}{ }^{2} / 2 \varepsilon_{0} \varepsilon$ when intrinsic layers at the junction. ${ }^{[2]}$ The latter is particularly important in organic solar cells, where the apparent $V_{b i}$ from MS depends on the active layer thickness. This was studied by Mingebach et al. ${ }^{[38]}$ who alternatively measured $V_{b i}$ by pulsed photocurrent. Also, the numerical simulations by Kirchartz et al. ${ }^{[36]}$ suggested discrepancies between the $V_{b i}$ apparent from MS and the simulated one, in organic solar cells.

In the case of heterostructures, the use of the one-sided abrupt (OSA) junction approximation should be justified (see Table 2.1). For this, the doping concentration of one of the charge carrier selective layers, $N_{D}$ or $N_{A}$, should be estimated and compared with that of the complete device $N$. For instance, in a typical PSCs the $\mathrm{TiO}_{2}$ doping density $N_{D}$ could be measured. If $N_{D} \gg N$, the use of the OSA equation would be justified and $N \approx N_{A}$. Otherwise, the other charge carrier selective layer should be checked. 
The estimation of the doping concentration for individual hole or electron selective layers, HSL or ESL respectively, can also be made by MS analysis independently of the complete solar cell. Here the main options are the p-n junction with a reference doped semiconductor, or the Schottky diode configuration. The latter is the archetype of the OSA approximation, where the depletion zone is only placed in the semiconductor and the opposite charge is at the surface of the metal or electrolyte solution. For instance, the Schottky diode mode in electrolyte solution method have been widely used with $\mathrm{TiO}_{2}$ thin films. ${ }^{[39-40]}$ Note that this is anyway a polemic approach, since the fabrication process itself modifies the defect densities.

Under illumination and around short-circuit and/or reverse bias an increase of the $C_{d l}$ occurs, as already commented in Section 2.6.1. Purposely, Zonno et al. ${ }^{[41]}$ illustrated the major contribution on the shape of the capacitance-voltage curve which can arise from the mobility of the blend in organic solar cells. They did analyzed the series and parallel modes of measuring the MS plot and discussed the relation $C_{s}=C_{p}+\omega^{-2} R_{p}{ }^{-2} C_{p}{ }^{-1}$.

In summary, the MS analysis is performed by measuring the capacitance from IS at a single frequency $f_{d l}$ while varying the DC voltage. Later, the $C_{s}$ or $C_{p}$ variants are assumed, and the doping density and built-in potentials are estimated from the linear fitting of the $C^{-2}(V)$ curve. The selection of $f_{d l}$ and the EC mode (series, parallel, or more complex) are of major attention here.

\subsubsection{Thermal admittance spectroscopy (TAS) measurement}

Already introduced in Section 2.6.2, defect concentrations $N_{t}$ of deep level trap states, at energy depth $E_{t}$ from the bands, affect the depletion layer capacitance under voltage perturbation. As the trapping-detrapping processes take place, the charge at the edge of the depletion zone is modified depending on the characteristic emission rate $\omega_{e m}=\omega_{0} \exp \left[-E_{t} / \mathrm{k}_{\mathrm{B}} \mathrm{T}\right]$ and the attempt-to-scape frequency $\omega_{0} \propto T^{2}$. The time/frequency and temperature dependency of the trap states charging/discharging are the core of the thermal admittance spectroscopy (TAS).

Under AC perturbation at frequency $\omega$ (see Section 3.3.1), the TAS explores the density-ofstates $g(E)$ conforming to defect levels over the change of the demarcation energy ${ }^{[42]}$

$$
E_{\omega}=k_{B} T \ln \left[\frac{\omega_{0}}{\omega}\right]
$$

The $E_{\omega}$ concept is also illustrated in the energy diagram example of Figure 3.5a. Assuming quasi-steady-state, the occupation of one defect level of energy $E_{t}$ above the top of the conduction band $E_{V}$ is defined by whether the Fermi level $E_{F}$ crosses the trap level $E_{t}$ at a given position of the semiconductor bulk. Upon bias perturbation, the defect state is incapable to follow the signal $\tilde{V}$ because $E_{\omega}$ locates far from $E_{t}$, in case of high frequencies $\left(\omega>\omega_{0}\right)$ or lesser temperatures.

The trap state contributes to the capacitance, in a cumulatively way, when the applied perturbation has a frequency lower than $\omega_{0}$. Then when $\omega \sim \omega_{\text {em }}\left(E_{\omega} \sim E_{t}\right)$ the maximum rate of de-trapping cycles occurs at the given temperature. ${ }^{[43]}$ For lower enough frequencies $\left(\omega<\omega_{e m}<\right.$ $\omega_{0}$ ) or higher enough temperatures, the steady state is achieved and the defect state occupancy changes in-phase with the AC modulation. This suppress further contribution to the low frequency capacitance spectra. Hence, the capacitance Bode plot high frequency plateau (see Figure 3.4d), 
may step a trap-related amount $\Delta C_{d l}$ towards low frequencies, as in Figure 3.5b. From the step $\Delta C_{d l}$, the electronic density of states can be found by deriving the capacitance spectrum $C(\omega)$ as $^{[44]}$

$$
g\left(E_{\omega}\right)=-\frac{\omega V_{b i}}{q d_{t} k_{B} T} \frac{\partial C(\omega)}{\partial \omega}
$$

Homogeneous trap density in the semiconductor bulk is assumed in Equation (3.5), taking $d_{t}$ as the thickness of the region/layer where the traps are placed. In heterostructures and/or p-i-n devices, the depletion zone width $w$ can be larger than $d_{t}$. Then, care must be taken and (3.5) should be effectively redefined, as well as in case of different defect distributions and band profiles. ${ }^{[44]}$
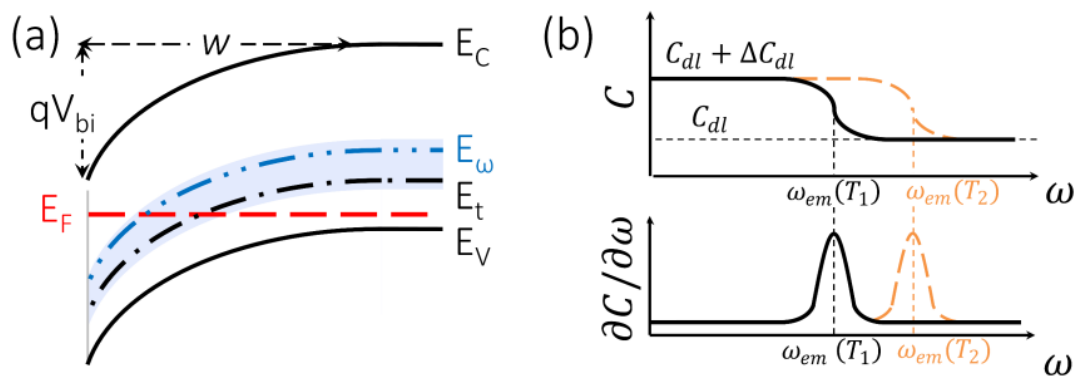

Figure 3.5. Thermal admittance spectroscopy measurement concept. (a) Quasiequilibrium energy diagram of the $\mathrm{p}$-side band bending in an abrupt junction with a bulk deep defect level of energy $E_{t}$ above the valence band under AC perturbation at frequency $\omega$ and corresponding demarcation energy $E_{\omega}$ above the valence band. (b) Capacitance spectrum step and corresponding derivative, proportional to the DOS, as temperature changes.

The peak of $\partial \mathrm{C}(\omega) / \partial \omega$ at the inflection point of the capacitance spectra step is illustrated in the lower panel of Figure 3.5b. Note that, from equation (3.5), the DOS is proportional to $\omega$. $\partial \mathrm{C}(\omega) / \partial \omega=\partial \mathrm{C}(\omega) / \partial \ln \omega$, which impose the logarithmic scale for identifying the peaks.

The total trap density can be studied by quite a few methods and more complex formalisms. ${ }^{[45-47]}$ Most basically, integrating Equation (3.5) over frequency (energy) at each temperature results in a proportionality with the excess trap-related capacitance step $\Delta C_{d l}$ over $C_{d l}$, or $C_{g}$ in full depletion, as

$$
N_{t}=\int g\left(E_{\omega}\right) d E_{\omega} \cong \frac{V_{b i}}{q d_{t}} \Delta C_{d l}
$$

The trap energy depth is evaluated from the slope of the Arrhenius plot of $\omega_{e m}$. The capacitance spectra is measured at different temperatures and for each one the $\omega_{e m}$ for the peak of $\mathrm{g}\left(\mathrm{E}_{\omega}\right)$ is taken. Typically, $E_{t}$ and $\sigma_{c s}$ are considered temperature independent parameters. Then, with $N_{V} v_{t h} \propto T^{2} \quad$ (see Section 2.6.2) the Arrhenius is plotted with $\ln \left[\omega_{e m} T^{-2}\right]$ as a function of $1 /\left(k_{B} T\right)$, and the slope and intercepts of the linear fitting gives $E_{t}$ and $\sigma_{c s}$, respectively. 
In summary, TAS consist in measuring IS spectra at different temperatures and analyzing the capacitance spectra. At each temperature, the emission rate is taken from the peaks of the capacitance frequency derivative, proportional to the DOS. The integration of the DOS at each temperature gives the total trap density, and the trap energy depth is estimated from the Arrhenius of emission rates vs temperature.

\subsubsection{Light modulated photocurrent and photovoltage spectroscopies}

The standard potentiostatic IS is a well-known characterization technique for studying the electrical properties of materials and devices. This includes any sample able to conduct some current under electric field stimulus. However, some samples are electrically sensitive to other stimulus, for instance light, which is the case of photovoltaic solar cells.

Under an AC light perturbation, photo-sensitive samples may respond in photocurrent and/or photovoltage ways. These are the concepts behind the light intensity modulated photocurrent and photovoltage spectroscopies, IMPS ${ }^{[48-59]}$ and IMVS ${ }^{[54-55,}{ }^{60-62]}$ respectively. The IMPS has recently gained attention in perovskite solar cells, principally approaching the short-circuit (SC) condition. ${ }^{[58-59,63-65]}$ IMVS and IMPS separately describe the current and voltage responsivities $\Psi_{J}$ and $\Psi_{V}$, respectively.

In principle, a small incident light power perturbation $\tilde{P}_{i n}(t)=\left|\tilde{P}_{\text {in }}\right| \exp [i \omega t]$ is applied to the given DC illumination $\bar{P}_{i n}$, as in Figure 3.6a. The total incident photon fluence in units of light power density results

$$
P_{\text {in }}=\bar{P}_{\text {in }}+\left|\tilde{P}_{\text {in }}\right| \exp [i \omega t]
$$

Consequently, both photocurrent and photovoltage signals can be recorded in a solar cells. At a given applied DC voltage $\bar{V}$, the measured current density would have a phase shift $\phi_{J}$, as schemed in Figure 3.6b. Then, similarly to (3.2), one obtains $\tilde{J}=|\tilde{J}| \exp \left[i \phi_{J}\right]$. Henceforward, one can define the current responsivity transfer function as

$$
\Psi_{J}(\omega)=\frac{\tilde{J}}{\tilde{P}_{i n}}=\frac{|\tilde{J}|}{\left|\tilde{P}_{i n}\right|} \exp \left[i \phi_{J}\right]
$$

Likewise, at open-circuit $(J=0)$ the cell may deliver a DC photovoltage $\bar{V}_{o c}$ due to the $\bar{P}_{\text {in }}$ with another term due to the AC perturbation, hence

$$
V_{o c}=\bar{V}_{o c}+\tilde{V}_{o c} \exp [i \omega t]
$$

The measured photovoltage signal phase-shifts $\phi_{V}$ as in Figure 3.6c. Then, taking the AC component as $\tilde{V}_{o c}=\left|\tilde{V}_{o c}\right| \exp \left[i \phi_{V}\right]$ allows to define the photovoltage responsivity transfer function as

$$
\Psi_{V}(\omega)=\frac{\widetilde{V}_{o c}}{\widetilde{P}_{i n}}=\frac{\left|\widetilde{V}_{o c}\right|}{\left|\tilde{P}_{i n}\right|} \exp \left[i \phi_{V}\right]
$$

Equations (3.8) and (3.10) are the fundamental definitions for IMPS and IMVS, respectively. Several authors have introduced these techniques before ${ }^{[48-51,57,61,66]}$ and many recent studies on organic, dye sensitized and perovskite solar cells have been reported..$^{[55-56,58,62,67]}$ 
The effect of the light perturbation in the $J-V$ characteristic of a photovoltaic cell under DC illumination is approximately a vertical shift, as presented in Figure 3.6d,e. The perturbation occurs in a third axis, $P_{i n}$, normal to the $J-V$ plane. For instance, at SC the IMPS is set at $\bar{V}=0$ (black thick dot in Figure 3.6d) and the sinus-like current is phase shifted in time, creating a Lissajous ellipses with the perturbation. IMPS can be used everywhere else in the $J-V$ curve, similarly to IS, but SC is its most common characterization condition.

(a)

(b)

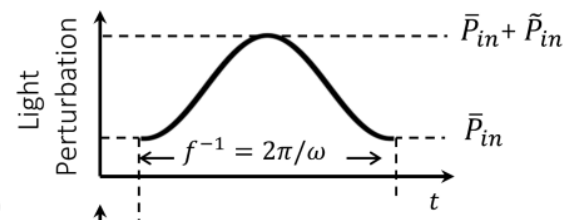

(c)
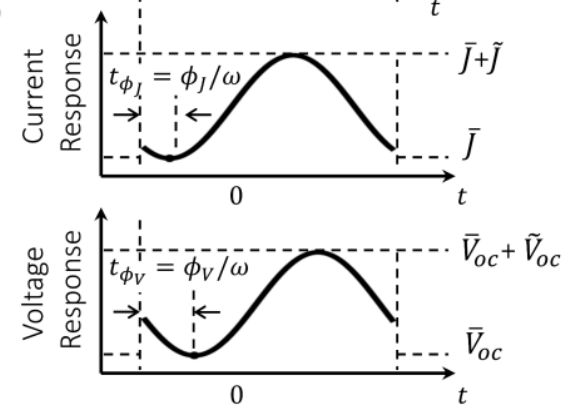

(d)

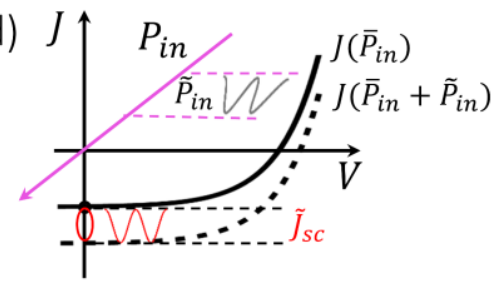

(e)

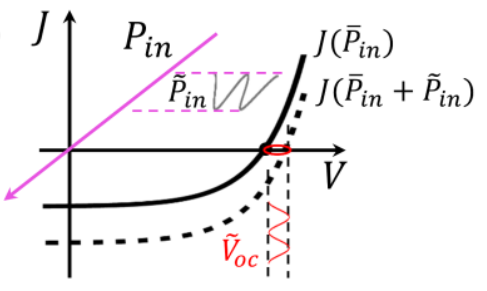

Figure 3.6. IMPS and IMVS concepts: (a) light perturbation, (b) current and (c) voltage responses and effects on the $J-V$ curve of a solar cell when (d) IMPS at short-circuit and (e) IMVS at open-circuit.

IMPS at SC checks the charge extraction mechanism in the absence of recombination current due to injection. If the incident light is monochromatic, IMPS can be used to measure the incidentphoton-to-converted-electron (IPCE) ratio, also known as photovoltaic external quantum efficiency:

$$
E Q E=\frac{N_{e l}}{N_{p h}}=\frac{h c}{q \lambda} \Psi_{J}
$$

Here $N_{e l}$ and $N_{p h}$ are the number of incident photons and converted/extracted electrons, respectively, $\Psi_{J}$ is the spectral responsivity in $\mathrm{A} \cdot \mathrm{W}^{-1}, \lambda$ the wavelength in $\mathrm{nm}$ and $h \cdot c / q=1240$ $\mathrm{W} \cdot \mathrm{nm} \cdot \mathrm{A}^{-1}$. Purposely, Ravishankar et al. ${ }^{[63]}$ have studied the frequency dependent behavior of EQE from IMPS in PSCs, under different DC illumination conditions, in comparison with chopped and steady-state instrumentations.

The total photocurrent that a solar cell can provide under an incident irradiance spectrum $\Gamma$, in units of $\mathrm{W} \cdot \mathrm{m}^{2} \cdot \mathrm{nm}^{-1}$, comes from the integration

$$
J_{s c}=\frac{q}{h c} \int E Q E(\lambda) \cdot \lambda \cdot \Gamma(\lambda) d \lambda=\int \Psi_{J}(\lambda) \cdot \Gamma(\lambda) d \lambda
$$


Most typically, the spectrum is assumed the standard AM1.5G reference. Note that equation (3.12) is meant to steady-state DC situation, which may be the low-frequency limit of $\Psi_{J}$ from IMPS.

On the other hand, IMVS can only be measured at open circuit (black thick dot in Figure 3.6e), differently to IMPS or IS. This is a conceptually important feature: IMPS and IS can measure quasi-open-circuit steady state by applying the voltage $\bar{V}$ that best matches the $\bar{V}_{\text {oc }}$ under $\bar{P}_{i n}$,with remaining currents between nanoamps and microamps. But IMVS sets open circuit, with zero currents, independently of the illumination DC or AC character. This means that the IMVS is a "purer" method to characterize charge recombination phenomena in solar cells.

The IMPS and IMVS spectra can be simulated via time-dependent solution of the transport equations or EC models in the frequency domain, similarly to IS. On the EC models, unlike IS, the inclusion of current sources as a new circuit element is needed.

In summary, IMVS and IMPS characterize the "ability" of the cell to create photocurrent and photovoltage, respectively, under light perturbation. Furthermore, IMPS is an useful tool for measuring EQE and evaluating the theoretical limit of $J_{S c}$ under a given spectrum.

\subsubsection{Instrumentation of impedance and light modulating spectroscopies}

As introduced in Section 3.3.1, equation (3.1), for the potentiostatic variant of IS, a voltage $\bar{V}$ is set constant and an AC perturbation $\tilde{V}$ is then applied. These functions require the use of a workstation including a potentiostat and an AC measurement unit. The measurements discussed in following chapters were correspondingly made with Autolab PGSTAT-30, Gamry Reference 3000, Biologic SP-200, and Zahner Zennium Pro workstations. In all these cases, the AC measurement unit was a frequency-response analyzer (FRA).

The general setup for measurement of IS, IMPS and IMVS is schemed in Figure 3.7a. Once the sample is connected, the entire measurement is controller from the computer (see 1 in Figure 3.7b). The initial perturbation parameters are set, and after the measurement the results are recorded.

The workstation and the sample are placed inside a grounded Faraday cage, as an anti-noise strategy (see 2 in Figure 3.7b). For IS measurements under DC illumination, as well as for IMPS and IMVS studies, the sample is set in an optical bench like in Figure 3.7c.

The potentiostat, inside the workstation, receives the instructions from the computer and set the DC voltage between the counter electrode (CE) and the working electrode (WE). Then the voltage is measured between the reference electrode (RE) and the working electrode, and rectified if needed. For DC measurements, the current through the working electrode is then measured and the information is sent to the computer. For AC measurements, the AC voltage perturbation is added between $\mathrm{CE}$ and WE, and the voltage signals between RE and WE, and the current signal through the WE are amplified and sent to the FRA. The FRA operate by correlating the studied signal with a reference signal, i.e. the measured signal is multiplied by a cosine and sine signal of the same frequency, and the product is time-integrated to obtain the real and imaginary parts. This information is then set to the computer. ${ }^{[26,28]}$ In the case of IMPS and IMVS, a second potentiostat 
(PP11 in the Zahner setup) controls the light source and the input current and voltage signals to the FRA are set conveniently proportional to the light intensity, photocurrent or photovoltage.

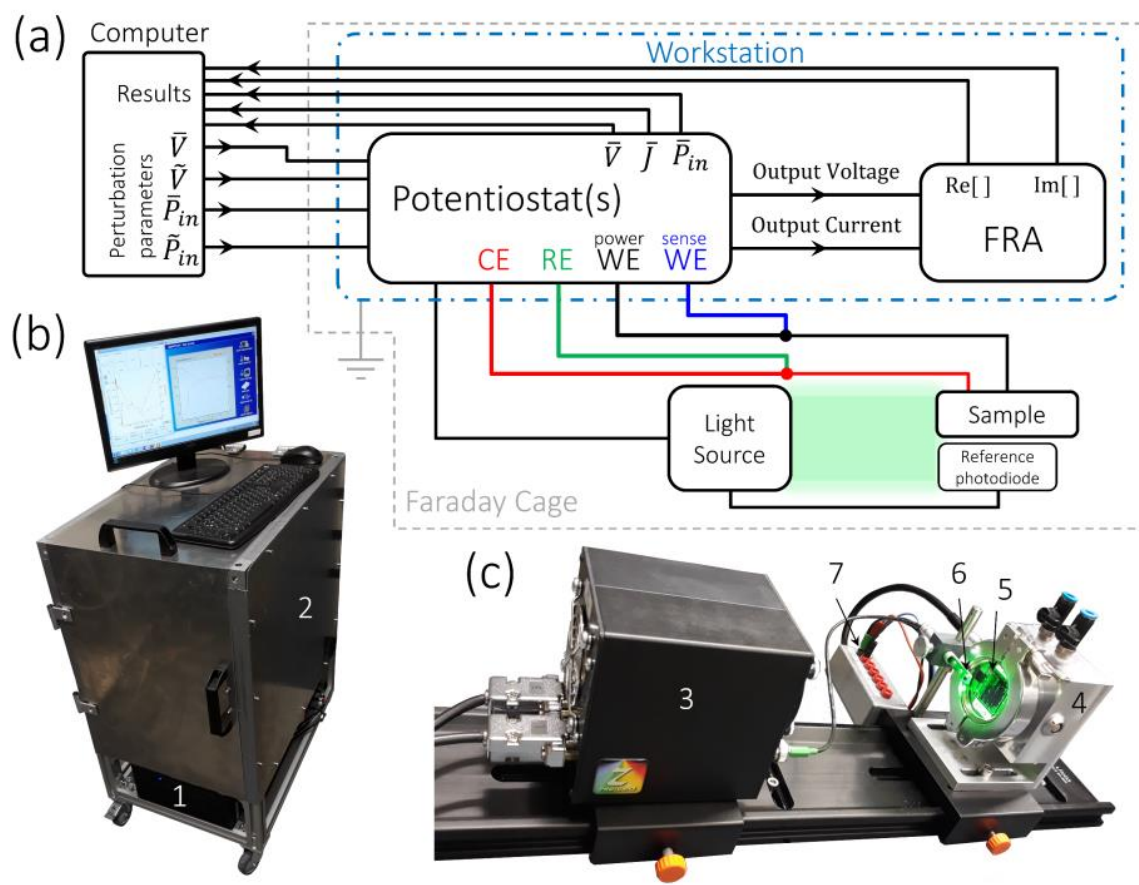

Figure 3.7. Impedance spectroscopy instrumentation: (a) schemed complete setup and pictures of (b) the exterior view of the and (1) computer, (2) Faraday cage and (c) the interior optical bench with (3) the light source box, (4) sample holder, (5) illuminated sample through the glass window, (6) reference photodiode, and (7) pixel connection selector/switcher.

In the optical bench of Figure 3.7c, the light source box (3) is set in front of the holder (4). The holder is dedicated to $2.00 \times 2.00 \mathrm{~cm}^{2}$ six pixels samples, including air isolation by continuous flux of $\mathrm{N}_{2}$ (see pipes in the top). The light goes through a glass window to be absorbed by the sample (5). Just outside the window, the reference photodiode (6) senses the incident illumination intensity giving feedback to the potentiostats. Each pixel of the sample can be plugged in the connection selector (7).

For temperature dependent measurements, like TAS, a similar configuration should be adopted, only that the holder may be specially modified for temperature control. The Zahner setup can be combined with the THMS600 stage from Linkam Scientific, as already reported. ${ }^{[68]}$ Alternatively, specialized setups can be used, like the Alpha-N analyzer with Quatro Cryosystem temperature controller from Novocontrol Technologies. 


\subsection{Sawyer-Tower circuit instrumentation}

Alternativelly to IS capacitance analysis, the charging transients of a two-electrode sample can be studied by means of the Sawyer-Tower (ST) circuit. ${ }^{[69-70]}$ The ST measurement is a classical method used to evaluate hysteretic loops of ferroelectric samples. The circuit is schemed in Figure 3.8a, where a signal generator applies a voltage $V_{a p p}$ to the series connection of two capacitances: the sample $C_{x}$ and a reference capacitor $C_{r e f}$. Once the sample is characterized by IS, and $C_{x}$ is known, the reference capacitor is selected so $C_{r e f} \gg C_{x}$. Thereupon, the charging process is going to be dominated by the smaller capacitor, where most of the $V_{a p p}$ drops, despite being the same for both. The charge is then measured in the reference capacitor while characterizing the sample, as

$$
Q_{S T}=C_{\text {ref }} V_{\text {ref }}=C_{x}\left(V_{\text {app }}-V_{\text {ref }}\right)
$$

being $V_{r e f}$ the voltage drop throught the reference capacitor, which is sampled by a high input impedance oscilloscope. For instance, an oscilloscope with typical $1 \mathrm{M} \Omega$ input impedance is not a proper instrument for highly resistive samples $(10-100 \mathrm{M} \Omega)$. Note that $C_{r e f}$ is in units of Farads, thus $Q_{S T}$ is in Coulombs, which makes the polarization at the sample as:

$$
\mathcal{P}=\frac{Q_{S T}}{A_{x}}
$$

in units of charge density $C \cdot \mathrm{cm}^{2}$, being $A_{x}$ the electrode area of the sample.

Typical polarization loops measured in the ST circuit from ferroelectric materials present a remnant polarization $\mathcal{P}_{r}$ at zero electric field after the loop is cycled below the spontaneous or saturation polarization, as in Figure 3.8b. In the absence of ferroelectric effect, a sample would have an RC couple whose ST response is also characteristic. Figure 3.8c shows typical pure linear (i) capacitor and (ii) resistor behaviors in the left pannel and more realistic (iii) capacitor with leakage currents in top right pannel. Thus, (iv) ferroelectric resisitive capacitor can shown an additional opening and sloping of the hysteresis loop by effects of the linear resistance and capacitanc, respectively, as in lower right pannel of Figure 3.8c. ${ }^{[71-72]}$

(a)

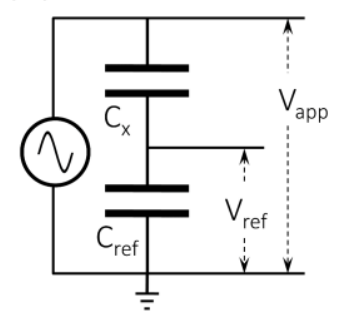

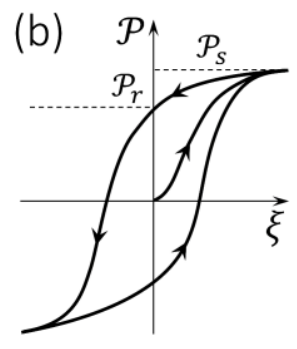
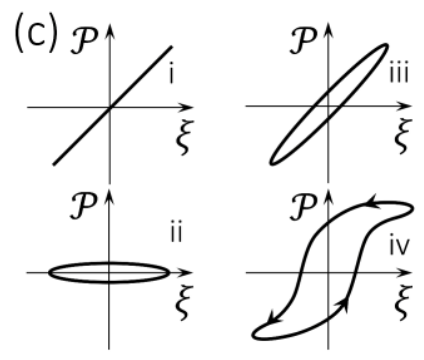

Figure 3.8. Sawyer-Tower (a) circuit, (b) typical ferroelectric hysteresis loop and (c) further patterns for the cases: (i) capacitor, (ii) resistor, (iii) resistive capacitor and (iv) ferroelectric resistive capacitor. 
The hysteresis, like that of Figure 3.8b, is the original fanily of phenomena which geave name to the anomalous current-voltage patterns in PSCs. The macroscopic polarization via ST has been already reported in PSCs, showing only capacitive features. ${ }^{[1,3]}$ The use of the ST setup for the measurements in following chapters included an Agilent 33220A 2MHz function arbitrary waveform generator and Hewlett Packard Infinium oscilloscope (500 MHz, $1 \mathrm{GSa} / \mathrm{s})$.

To sum up, the ST circuit is an useful tool for studying the evolution of charging phenomena. It illustrates ferroelectric, capacitive and/or resistive behaviors from the polarization loops.

\section{References}

[1] O. Almora, I. Zarazua, E. Mas-Marza, I. Mora-Sero, J. Bisquert, G. Garcia-Belmonte, J. Phys. Chem. Lett. 6 (2015), 1645. https://doi.org/10.1021/acs.jpclett.5b00480

[2] O. Almora, M. García-Batlle, G. Garcia-Belmonte, J. Phys. Chem. Lett. 10 (2019), 3661. https://doi.org/10.1021/acs.jpclett.9b00601

[3] M. Coll et al., J. Phys. Chem. Lett. 6 (2015), 1408. https://doi.org/10.1021/acs.jpclett.5b00502

[4] O. Almora, C. Aranda, E. Mas-Marzá, G. Garcia-Belmonte, Appl. Phys. Lett. 109 (2016), 173903. https://doi.org/10.1063/1.4966127

[5] O. Almora, A. Guerrero, G. Garcia-Belmonte, Appl. Phys. Lett. 108 (2016), 043903. https://doi.org/10.1063/1.4941033

[6] J. Carrillo et al., Adv. Energy Mater. 6 (2016), 1502246 https://doi.org/10.1002/aenm.201502246

[7] O. Almora, C. Aranda, I. Zarazua, A. Guerrero, G. Garcia-Belmonte, ACS Energy Lett. 1 (2016), 209. https://doi.org/10.1021/acsenergylett.6b00116

[8] M. Valles-Pelarda et al., J. Phys. Chem. Lett. 7 (2016), 4622. https://doi.org/10.1021/acs.jpclett.6b02103

[9] S. Ravishankar et al., J. Phys. Chem. Lett. 8 (2017), 915. https://doi.org/10.1021/acs.jpclett.7b00045

[10] O. Almora, C. Aranda, G. Garcia-Belmonte, J. Phys. Chem. C 122 (2018), 13450. https://doi.org/10.1021/acs.jpcc.7b11703

[11] O. Almora et al., Nano Energy 48 (2018), 63. https://doi.org/10.1016/j.nanoen.2018.03.042

[12] O. Almora et al., Sol. Energy Mater. Sol. Cells 195 (2019), 291.

https://doi.org/10.1016/j.solmat.2019.03.003

[13] O. Almora, G. Garcia-Belmonte, Solar Energy 189 (2019), 103.

https://doi.org/10.1016/j.solener.2019.07.048

[14] O. Almora et al., Nano Energy submitted (2020), https://arxiv.org/abs/1911.05440.

[15] K. T. Cho et al., Energy Environ. Sci. 11 (2018), 952. https://doi.org/10.1039/C7EE03513F

[16] A. Guerrero et al., Adv. Energy Mater. 8 (2018), 201703376.

https://doi.org/10.1002/aenm.201703376

[17] M. S. Jamal et al., Renewable Sustainable Energy Rev. 98 (2018), 469. https://doi.org/10.1016/j.rser.2018.09.016

[18] Z. Li et al., Nat. Rev. Mater. 3 (2018), 18017. https://doi.org/10.1038/natrevmats.2018.17

[19] N. Pellet et al., Prog. Photovoltaics 25 (2017), 942. https://doi.org/10.1002/pip.2894

[20] L. Rakocevic et al., Sol. RRL 3 (2019), 1800287. https://doi.org/10.1002/solr.201800287

[21] D. D. Macdonald, Electrochimica Acta 51 (2006), 1376.

https://doi.org/10.1016/j.electacta.2005.02.107

[22] J. Bisquert, F. Fabregat-Santiago, in Dye-sensitized solar cells, (Ed: K. Kalyanasundaram), CRC Press, Lausanne (Switzerland) 2010, 457.

[23] O. Almora, L. G. Gerling, C. Voz, R. Alcubilla, J. Puigdollers, G. Garcia-Belmonte, Sol. Energy Mater. Sol. Cells 168 (2017), 221. https://doi.org/10.1016/j.solmat.2017.04.042

[24] J. Bisquert, Phys. Chem. Chem. Phys. 5 (2003), 5360. https://doi.org/10.1039/B310907K

[25] C. A. Schiller, R. Kaus, ECS Transactions 25 (2010), 49. https://doi.org/10.1149/1.3324743

[26] V. F. Lvovich, Impedance Spectroscopy. Applications to Electrochemical and Dielectric

Phenomena, John Wiley \& Sons, Inc., Hoboken, New Jersey, 2012.

[27] J. Jamnik, J. Maier, Phys. Chem. Chem. Phys. 3 (2001), 1668. https://doi.org/10.1039/B100180I 
[28] A. Lasia, Electrochemical Impedance Spectroscopy and its Applications, Springer, New York, 2014. ISBN: 9781461489337.

[29] A. Todinova et al., ChemElectroChem 4 (2017), 1. https://doi.org/10.1002/celc.201700498

[30] P. Yadav, K. Pandey, V. Bhatt, M. Kumar, J. Kim, Renewable Sustainable Energy Rev. 76 (2017), 1562. https://doi.org/10.1016/j.rser.2016.11.205

[31] E. von Hauff, J. Phys. Chem. C 123 (2019), 11329. https://doi.org/10.1021/acs.jpcc.9b00892

[32] J. A. Carr, S. Chaudhary, Appl. Phys. Lett. 100 (2012), 213902. https://doi.org/10.1063/1.4720403

[33] G. Jarosz, J. Non-Cryst. Solids 354 (2008), 4338. https://doi.org/10.1016/j.jnoncrysol.2008.06.077

[34] G. Jarosz, Appl. Phys. Lett. 102 (2013), 076101. https://doi.org/10.1063/1.4793133

[35] K.-M. Guenther, H. Witte, A. Krost, S. Kontermann, W. Schade, Appl. Phys. Lett. 100 (2012), 042101. https://doi.org/10.1063/1.3679380

[36] T. Kirchartz et al., J. Phys. Chem. C 116 (2012), 7672. https://doi.org/10.1021/jp300397f

[37] A. Nigam, M. Premaratne, P. R. Nair, Org. Electron. 14 (2013), 2902.

https://doi.org/10.1016/j.orgel.2013.08.005

[38] M. Mingebach, C. Deibel, V. Dyakonov, Physi. Rev. B 84 (2011), 153201.

https://doi.org/10.1103/PhysRevB.84.153201

[39] A. Guerrero, E. J. Juarez-Perez, J. Bisquert, I. Mora-Sero, G. Garcia-Belmonte, App. Phys. Lett. 105 (2014), 133902. https://doi.org/10.1063/1.4896779

[40] F. Fabregat-Santiago, G. Garcia-Belmonte, J. Bisquert, P. Bogdanoff, A. Zaban, J. Electrochem. Soc. 150 (2003), E293. https://doi.org/10.1149/1.1568741

[41] I. Zonno, A. Martinez-Otero, J.-C. Hebig, T. Kirchartz, Phys. Rev. Appl. 7 (2017), 034018. https://doi.org/10.1103/PhysRevApplied.7.034018

[42] S. S. Hegedus, E. A. Fagen, J. Appl. Phys. 71 (1992), 5941. https://doi.org/10.1063/1.350444

[43] J. A. Carr, M. Elshobaki, S. Chaudhary, Appl. Phys. Lett. 107 (2015), 203302. https://doi.org/10.1063/1.4936160

[44] T. Walter, R. Herberholz, C. Müller, H. W. Schock, J. Appl. Phys. 80 (1996), 4411. https://doi.org/10.1063/1.363401

[45] P. M. Mooney, in Semiconductors and Semimetals, Vol. 51 (Ed: M. Stavola), Elsevier, 1999, 93. https://doi.org/10.1016/S0080-8784(08)62975-0

[46] P. Blood, J. W. Orton, The Electrical Characterization of Semiconductors: Majority Carriers and Electron States, Academic Press, Oxford, England, 1992. ISBN: 9780125286275.

[47] J. Auth, Phys. Status Solidi B 27 (1968), 653. https://doi.org/10.1002/pssb.19680270222

[48] E. Kamieniecki, J. Vac. Sci. Technol. 20 (1982), 811. https://doi.org/10.1116/1.571491

[49] E. Kamieniecki, J. Appl. Phys. 54 (1983), 6481. https://doi.org/10.1063/1.331876

[50] R. Peat, L. M. Peter, J. Electroanal. Chem. Interfacial Electrochem. 228 (1987), 351. https://doi.org/10.1016/0022-0728(87)80117-1

[51] E. A. Ponomarev, L. M. Peter, J. Electroanal. Chem. 396 (1995), 219. https://doi.org/10.1016/0022-0728(95)04115-5

[52] L. Dloczik et al., J. Phys. Chem. B 101 (1997), 10281. https://doi.org/10.1021/jp972466i

[53] P. E. de Jongh, D. Vanmaekelbergh, J. Phys. Chem. B 101 (1997), 2716. https://doi.org/10.1021/jp962226n

[54] G.-O. Kim, Bull. Korean Chem. Soc. 33 (2012), 469. https://doi.org/10.5012/BKCS.2012.33.2.469

[55] A. Pockett et al., J. Phys. Chem. C 119 (2015), 3456. https://doi.org/10.1021/jp510837q

[56] Y. Gao, A. J. Wise, A. K. Thomas, J. K. Grey, ACS Appl. Mater. Interfaces 8 (2016), 285. https://doi.org/10.1021/acsami.5b08724

[57] L. Bertoluzzi, J. Bisquert, J. Phys. Chem. Lett. 8 (2017), 172. https://doi.org/10.1021/acs.jpclett.6b02714

[58] S. Ravishankar, C. Aranda, S. Sanchez, J. Bisquert, M. Saliba, G. Garcia-Belmonte, J. Phys. Chem. C 123 (2019), 6444. https://doi.org/10.1021/acs.jpcc.9b01187

[59] S. Ravishankar, A. Riquelme, S. K. Sarkar, M. Garcia-Batlle, G. Garcia-Belmonte, J. Bisquert, J. Phys. Chem. C 123 (2019), 24995. https://doi.org/10.1021/acs.jpcc.9b07434

[60] J. Krüger, R. Plass, M. Grätzel, P. J. Cameron, L. M. Peter, J. Phys. Chem. B 107 (2003), 7536. https://doi.org/10.1021/jp0348777 
[61] Y. T. Set, B. Li, F. J. Lim, E. Birgersson, J. Luther, Appl. Phys. Lett. 107 (2015), 173301. https://doi.org/10.1063/1.4934920

[62] X. Chen, Y. Shirai, M. Yanagida, K. Miyano, Phys. Chem. Chem. Phys. 20 (2018), 17918. https://doi.org/10.1039/C8CP01227J

[63] S. Ravishankar, C. Aranda, P. P. Boix, J. A. Anta, J. Bisquert, G. Garcia-Belmonte, J. Phys. Chem. Lett. 9 (2018), 3099. https://doi.org/10.1021/acs.jpclett.8b01245

[64] Q. Cui et al., Nanoscale 9 (2017), 18897. https://doi.org/10.1039/C7NR05687G

[65] D. Prochowicz et al., ACS Omega 4 (2019), 16840. https://doi.org/10.1021/acsomega.9b01701

[66] J. Li, L. M. Peter, J. Electroanal. Chem. Interfacial Electrochem. 193 (1985), 27. https://doi.org/10.1016/0022-0728(85)85050-6

[67] J. C. Byers, T. Heiser, M. Skorobogatiy, O. A. Semenikhin, ACS Appl. Mater. Interfaces 8 (2016), 28789. https://doi.org/10.1021/acsami.6b12345

[68] C. Zhang et al., ACS Appl. Mater. Interfaces 11 (2019), 18555. https://doi.org/10.1021/acsami.8b22539

[69] C. Sawyer, C. Tower, Physical Review 35 (1930), 269. http://link.aps.org/doi/10.1103/PhysRev.35.269

[70] K. M. Rabe, M. Dawber, C. Lichtensteiger, C. H. Ahn, J.-M. Triscone, in Physics of Ferroelectrics. A Modern Perspective, (Eds: K. M. Rabe, C. H. Ahn, J.-M. Triscone), Springer, Berlin 2007, 388. https://doi.org/10.1007/978-3-540-34591-6

[71] H. Roetschi, J. Sci. Instrum. 39 (1962), 152. https://doi.org/10.1088/0950-7671/39/4/304

[72] N. W. Schubring, J. P. Nolta, R. A. Dork, Revi. Sci. Instrum. 35 (1964), 1517. https://doi.org/10.1063/1.1719196 


\section{Chapter 4. Dark characterizations and modeling}

In the previous chapters, the current-voltage characteristic, capacitance and impedance spectroscopy of solar cells were introduced, among other concepts. The focus of the following chapters is set on the understanding of the anomalous current-voltage $(J-V)$ curve hysteresis and capacitive features of perovskite solar cells (PSCs). In that direction, a logical order is set by studying the system from lower to higher complexity.

In this chapter, a set of electrical experiments in dark condition are discussed. First, the bulk methylammonium lead iodide (MAPI) material is electrically characterized by impedance spectroscopy (IS) and Sawyer-Tower (ST) approaches, and later the complete PSC device. Dark $J-V$ characteristics depending on the voltage sweep scan rate and temperature are analyzed later, and the IS measurements are approached, first as a function of temperature and later in a range of different direct current mode (DC) biases.

\subsection{Electric characterization of MAPI pellets}

As a first step in the understanding of PSCs we study their core material: the perovskite absorber. The samples under consideration here are $\mathrm{CH}_{3} \mathrm{NH}_{3} \mathrm{PbI}_{3}$ pellets of $1.33 \mathrm{~cm}$ diameter, thicknesses below 0.3-1.0 mm, and crystal sizes thicker than $400 \mu \mathrm{m}$, as illustrated in Figure 4.1a. The fabrication details and structural characterization can be found in the original article. ${ }^{[1]}$

The focus of this section is set on the conductivity and dielectric properties. From the IS formalism, these are magnitudes mainly related with the real and imaginary parts of the impedance, energy dissipation and storage, respectively. However, they can be approached by alternative methods and/or in different timescales.

\subsubsection{Capacitance and conductivity spectra}

Geometrical capacitance is the basic signal which can be recorded from a single material between symmetric electrodes. Thus, the relative permittivity is the main factor to consider at frequencies between $1 \mathrm{kHz}$ and $1 \mathrm{MHz}$. At lower frequencies, electrode effects are typical.

The dielectric function of MAPI has been theoretically obtained from first principles by many authors, ${ }^{[2-11]}$ with a significant scattering between 5 and 38 for the value of the static dielectric constant at room temperature. Experimental analyses have also being reported by means of ultraviolet-visible optical absorption and transmittance spectroscopies, ${ }^{[12-13]}$ spectroscopic ellipsometry and spectrophotometry. ${ }^{[13-15]}$

In the tetragonal phase ( 160-330 K), Onoda et al. ${ }^{[16]}$ and Mohanty et al. ${ }^{[17]}$ measured static dielectric constant at $1 \mathrm{MHz}$ by IS versus temperature with $\varepsilon \sim 60-120$ and thermal gradients $\partial \varepsilon / \partial T \sim-0.3 \mathrm{~K}^{-1}$. At room temperature, Ding et al. ${ }^{[18]}$ showed $\varepsilon$ spectrum above 50 too. At 20 $\mathrm{kHz}$, Fabini et al. ${ }^{[19]}$ obtained $\varepsilon \sim 35-50$. In a range of $40 \mathrm{~K}$ above room condition, Yang et al. ${ }^{[20]}$ found $\varepsilon \sim 31-34$, with $\partial \varepsilon / \partial T \sim-0.05 \mathrm{~K}^{-1}$. Anomalously, Slonopas et al. ${ }^{[21-22]}$ found $\varepsilon \sim 10^{4}$ in a wide plateau spectra between $10 \mathrm{~Hz}$ and $100 \mathrm{kHz}$, and in the range 100-350 K.

The equilibrium capacitance spectra in the range 190-295 K are shown in Figure 4.1b. At frequencies below $10 \mathrm{~Hz}$, a clear thermally activated process occurs, most likely related with 
electrode polarization. The experimental data were fitted (dots and lines in Figure 4.1c, respectively) to

$$
C=C_{g}+C_{e l}\left(1+\left(\frac{\omega}{\omega_{e l}}\right)^{2}\right)^{-1}
$$

where $C_{g}$ is the geometrical capacitance after (2.37), $C_{e l}$ the electrode polarization capacitance and $\omega_{e l}$ the characteristic activation frequency. Equation (4.1) resembles the Beamount-Jacobs electrode polarization model, ${ }^{[23-24]}$ and the Maxwell-Wagner-Sillars interfacial polarization model $^{[21-22,25]}$ has also been suggested. However, none of these models reproduce the frequency and temperature trends at the same time. Less predominant power laws cannot be neglected, but from the Arrhenius of Figure 4.1c it looks that $C_{e l} \propto \exp \left[-E_{C} / \mathrm{k}_{\mathrm{B}} \mathrm{T}\right]$ with $E_{C} \cong 384 \mathrm{meV}$, and $\omega_{e l} \propto \exp \left[-E_{\omega} / \mathrm{k}_{\mathrm{B}} \mathrm{T}\right]$ with $E_{\omega} \cong 159 \mathrm{meV}$.

The empirical frequency parametrization of equation (4.1), with the thermal activation behaviors as Figure 4.1c, can be understood in terms of a hopping-mediated electrode polarization processes where the low frequency electrode polarization capacitance results ${ }^{[26]}$

$$
C=C_{g}+\frac{N_{e l} q^{2} \omega_{H 0}}{m^{*} d \omega_{s 0}{ }^{3}} \exp \left[-\frac{\left(E_{H}-3 E_{s}\right)}{k_{B} T}\right]\left(1+\left(\frac{\omega}{\omega_{s}}\right)^{2}\right)^{-1}
$$

Here $q$ is the elementary charge, $d$ is the distance between electrodes, $k_{B} T$ the thermal energy, $N_{e l}$ the charge carrier density towards the electrodes, $m^{*}$ the effective mass of the charge carriers, $\omega_{H 0}$ and $\omega_{s 0}$ are the characteristic frequencies for quantum hopping and classical drifting between hopping sites, and $E_{H}$ and $E_{S}$ are the activation energies for hopping and charge carrier classical drifting between hopping sites. Equaling (4.1) to (4.2) results in $\omega_{e l}=\omega_{s}$, making $E_{\omega}=E_{s} \cong 159 \mathrm{meV}$ and $E_{C}=E_{H}-3 E_{s}$, for $E_{H} \cong 861 \mathrm{meV}$. The latter, also approach the theoretical estimations for activation energies of methylammonium vacancies. ${ }^{[27]}$

Note that Equation (4.2) relates two conductivity processes with activation energies $E_{H}$ and $E_{S}$ that could relate both electronic charge carriers scattering and hopping. In this case one could consider the presence of mobile ions to be proportional to the density of hopping sites towards the electrodes. Alternatively, one could assume that both processes are due to the movement of ions or a combination of drifted mobile ions with activation energy $E_{S}$ creating electronic hopping with activation energies $E_{H}$.

At frequencies above $10 \mathrm{kHz}$, nearly frequency-independent plateaus are found in the measured temperature range. The permittivity is shown to decrease with temperature as $\partial \varepsilon / \partial T \sim-0.03 \mathrm{~K}^{-1}$ in Figure 4.1d.

The DC conductivity $\sigma_{d c}$ of MAPI has been reported by several authors by means of four probe contacts measurements ${ }^{[28]}$ or two electrode contacts, in DC mode ${ }^{[18,29-30]}$ or from the low frequency limit of IS measurements. ${ }^{[20]}$ On the other hand, the AC conductivity has been only described by Sheikh et al., ${ }^{[31-32]}$ who identified up to two main regimes at intermediate and higher frequencies, above $\sigma_{d c}$.

The conductivity spectra are shown in Figure 4.1e with a strong thermal dependency within the tetragonal phase range and up to three power law frequency regimes. The experimental data were parametrized (dots and lines in Figure 4.1e, respectively) to the general Jonscher's 'universal' dielectric frequency response ${ }^{\text {[3-35] }}$ 


$$
\sigma=\sigma_{d c}\left(1+\left(\frac{\omega}{\omega_{\sigma}}\right)^{m_{\sigma}}\right)
$$

where $\omega_{\sigma}$ is a characteristic conductivity activation frequency and, well-known in disordered solids ${ }^{[36-38]}$ the power parameter is predicted $0.5 \leq m_{\sigma} \leq 1.0$ by several hopping models ${ }^{[39-42]}$ in ionic conducting glasses, while the diffusion-controlled relaxation model for ionic transport in glasses predicts $0<m_{\sigma}<0.5 .^{[43]}$

(a)
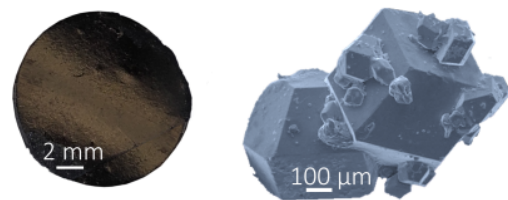

(b)

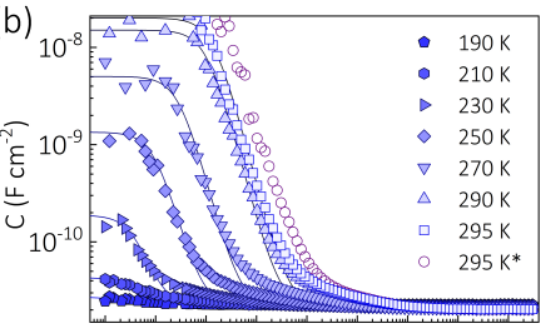

$10^{-2} 10^{-1} 10^{0} 10^{1} 10^{2} 10^{3} 10^{4} 10^{5} 10^{6}$

$\mathrm{f}(\mathrm{Hz})$

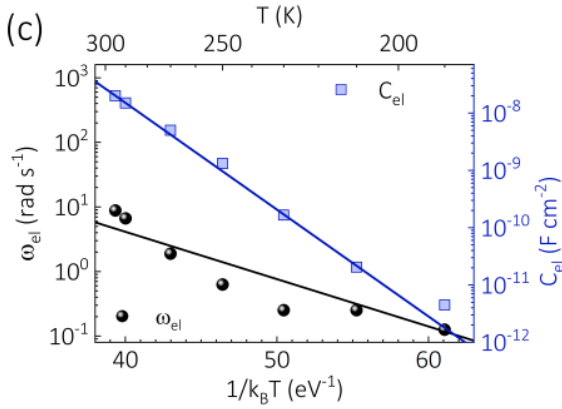

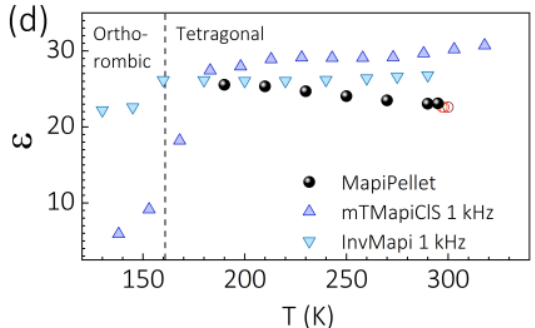
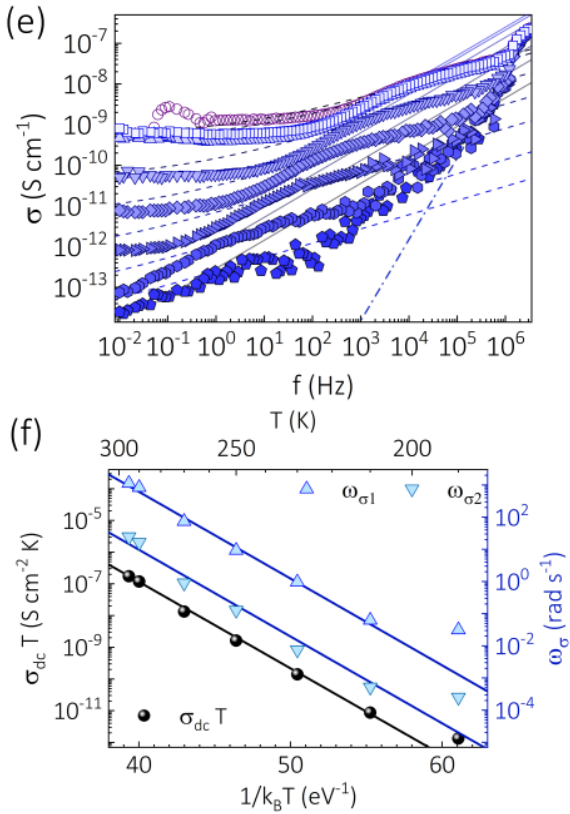

Figure 4.1. IS characterization of MAPI pellets as a function of temperature, in dark equilibrium. Representative (a) picture and SEM image of the samples. Adapted from ref. [1], Copyright 2016, AIP Publishing LLC. (b) capacitance spectra, (c) parametrized Arrhenius plot of capacitances and activation frequencies, (d) dielectric constant, (e) conductivity spectra and (f) parametrized DC conductivities and respective activation frequencies. Dots and lines are experimental data and fittings, respectively, in (b, e). The asterisks in (b) indicate that extra $1.0 \mathrm{~V}$ bias was applied. 
From the literature, Sheikh et al. ${ }^{[31]}$ reported $m_{\sigma}$ as 1.0 and 0.36 from intermediate and higher frequencies in $\mathrm{CH}_{3} \mathrm{NH}_{3} \mathrm{PbI}_{3}$. With a single power law, $m_{\sigma} \sim 0.92$ for $\mathrm{CH}_{3} \mathrm{NH}_{3} \mathrm{PbI}_{3}$, and $m_{\sigma} \sim 0.89$ for $\mathrm{CH}_{3} \mathrm{NH}_{3} \mathrm{PbBr}_{3}$ have also been reported. ${ }^{[32]}$ For inorganic perovskites, nanocrystalline $\mathrm{CsPbBr}_{3}$ thin films ${ }^{[44-45]}$ have shown $m_{\sigma} \sim 0.7-1.0$ at room temperature and $m_{\sigma} \sim 0.3$ above $400 \mathrm{~K}$ while, another study ${ }^{[46]}$ showed a power as high as $m_{\sigma} \sim 1.7$ from cubic shape $\mathrm{CsPbBr} 3$ particles of up to $3 \mu \mathrm{m}$. Furthermore, a temperature dependency of $m_{\sigma}$ between 0.7 and 1.0 has been reported for $\mathrm{CsPbI}_{3}$ microwires in the range 400-700 K. ${ }^{[4]}$

At the lowest frequencies, the DC conductivity in hopping theory results ${ }^{[34,48]}$

$$
\sigma_{d c}=\frac{N_{\sigma} q^{2} \lambda_{\sigma}{ }^{2}}{6 k_{B} T} \omega_{\sigma}
$$

where $\lambda_{\sigma}$ is the hopping distance, $N_{\sigma}$ the ion defect density and the effective jump rate $\omega_{\sigma}$ depends on whether the electron-phonon coupling is strong or weak. In the case of strong coupling ${ }^{[48]}$

$$
\omega_{\sigma}=\omega_{\sigma 0} \exp \left[-\frac{E_{\sigma}}{k_{B} T}\right]
$$

where $\omega_{\mathrm{h} 0}$ is the effective attempt-to-jump frequency and $E_{\sigma}$ is the effective activation energy. The latter parameter is composed at the same time by several contributions as the energy difference between hopping sites, ${ }^{[48]}$ and the free energies of creation and migration of charge carriers. ${ }^{[34]}$ The fitted $\sigma_{d c}$ values are shown to follow (4.4) with (4.5) and $E_{\sigma} \approx 629 \mathrm{meV}$ in Figure 4.1f. Note that this activation energy approaches theoretical predictions for methylammonium vacancies. ${ }^{[27]}$ Other similar analyses on $\sigma_{d c}$ in MAPI have shown $E_{\sigma}$ in the range $390-430 \mathrm{meV},{ }^{[20,29]}$ which most likely relates with iodide vacancies and/or interstitial methylammonium. ${ }^{[27]}$

At the largest frequencies the conductivity spectra seem to converge to $m_{\sigma}=2$, presented with a dot-dashed line in Figure 4.1e. At intermediate and lower frequencies the conductivity behaves $m_{\sigma 2}=0.35$ and $m_{\sigma 1}=0.7$, as illustrated with dashed and solid lines in Figure 4.1e, respectively. Correspondingly, the activation frequencies $\omega_{\sigma 1}$ and $\omega_{\sigma 2}$ are presented in Figure 4.1f, showing a clear proportionality with $\omega_{\sigma}$ as (4.5) with $E_{\sigma} \approx 629 \mathrm{meV}$ too.

These results suggest that the concentration of mobile ions can be proportional to the number of hopping sites, and/or a close connection between drifted mobile ions and hopping electrons could occur. Also, the presence of space charges at the electrode interfaces are of major significance in the electrical response of MAPI based samples.

\subsubsection{Sawyer-Tower transients experiments}

The bias effect seems to enhance the electrode capacitance, as evident with open circles in Figure 4.1b,e. Alternatively to the IS approach, the ST setup (see Section 3.4) allows to study both AC responses to sinusoidal perturbations and transient responses to step perturbations. Also, for particular instrumentation reasons, larger biases were available from ST than from IS setup.

Sinusoidal $V_{a p p}$ in the ST circuit to MAPI pellets samples at room temperature results in typical ellipses with more resistive or capacitive character as the frequency decrease or increase, respectively, as in Figure 4.2a. ${ }^{[49-50]}$ Similar results has been reported from the PUND (positive up and negative down) method. ${ }^{[51]}$ Importantly, as lower frequencies enhance the resistive character of the signal, one must take care of identifying the actual features related with the 
sample. Note that it could be the case that the voltage drop in the sample is due to the resistance and not due to the lower capacitance. Validation experiments are advised, as in the original

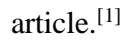

Rectangular pulses of height $V_{a p p}$ (top of Figure 4.2b) are applied by the signal generator to the ST circuit. The resulting signal $V_{r e f}$ is sampled by the osciloscope in the reference capacitor. Given the larger capacitance of the reference, $V_{r e f}$ characterizes the sample and shows a general shape like in the botton of Figure 4.2b. There, two main exponentials with different response times can be identified and fitted to:

$$
Q=Q_{H L}\left(1-\exp \left[-t / \tau_{H L}\right]\right)+Q_{\text {diff }}\left(1-\exp \left[-t / \tau_{\text {diff }}\right]\right)
$$

The first exponential term in (4.6) can be associated to Helmholtz layer dielectric response (expressed by $Q_{H L}$ and $\tau_{H L}$ ). On the other hand, ionic charging at a Gouy-Chapman diffuse layer (with parameters $Q_{\text {diff }}$ and $\tau_{\text {diff }}$ ) can be connected to the second term. Both polarization mechanisms comprise the double-layer structure of charge storage in the Stern model, most typically applied to semiconductor/electrolite systems, e.g. supercapacitors. ${ }^{[52-54]}$ The experimental charging of the sample is shown in two time scales in Figure 4.2c,d.

The faster charging is illustrated in Figure 4.2c, after discarding $C_{g}$ effects from the sample (see Figure 4.1b) and/or the reference capacitor (see ref. ${ }^{[1]}$ ). The fitted $Q_{H L}$ values exhibit a linear behavior as a function of $V_{a p p}$ in Figure 4.2e, whose slope may be the lower capacitance limit due to the effect of the ohmic voltage drop in the sample and/or reference capacitor effects. For example, the tens of $\mathrm{nF} \cdot \mathrm{cm}^{2}$ at lower frequencies in Figure $4.1 \mathrm{~b}$ could be justified with Helmholtz layers thinner than $200 \mathrm{~nm}$.

When a larger sampling window is considered in the osciloscope, one can observe up to tens of seconds, as in Figure 4.2d. These slower dynamic processes has also been detected by other techniques like transient photocurrent and photovoltage decays, in PSCs where the MAPI layer is typically thinner $(<1 \mu \mathrm{m}) \cdot{ }^{[55-58]}$ In the case of the MAPI pellet, the slower charging is correlated with the accumulation of mobile ions forming a diffuse layer towards the electrode interfaces. ${ }^{[50]}$ The electrode polarization was already tackled in the previous section from the capacitance spectra, and from Figure 4.2e one can see how $Q_{\text {diff }}$ deviates from a linear trend and results larger than $Q_{H L}$ as the aplied voltage increases. This behavior can be fitted to the Gouy-Chapman theory, ${ }^{[59]}$ resulting

$$
Q_{\text {diff }}=\frac{\varepsilon_{0} \varepsilon}{L_{D}} \frac{2 k_{B} T}{q} \sinh \left[\frac{q V_{\text {diff }}}{2 k_{B} T}\right]
$$

where $L_{D}$ is the Debye length after (2.44) and $V_{\text {diff }}$ is the effective fraction of $V_{a p p}$ which is droping at the diffussion layer. Note that $V_{\text {diff }}$ is the potential difference betwenn electrodes if ohmic looses, or any other voltage drops, can be neglected. The fitting of charge densities in Figure 4.2e suggest $V_{\text {diff }}$ to be around 3\% of $V_{\text {app }}$, and a Debye length thicker than $200 \mathrm{~nm}$.

The ionic charge density determined from Equation (2.44) gives around $10^{13} \mathrm{~cm}^{-3}$. This mobile ion concentration is related to the higher cristallinity of the MAPI pellet samples, in comparison with the thin films in PSCs. The preparation method for the MAPI pellets allows synthesizing large crystals of the order of the sample thickness (see Figure 4.2a). 

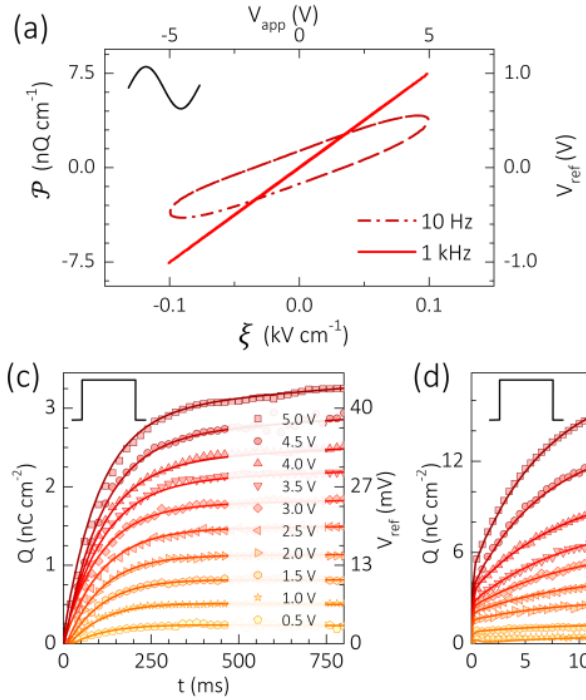

(d) (b)
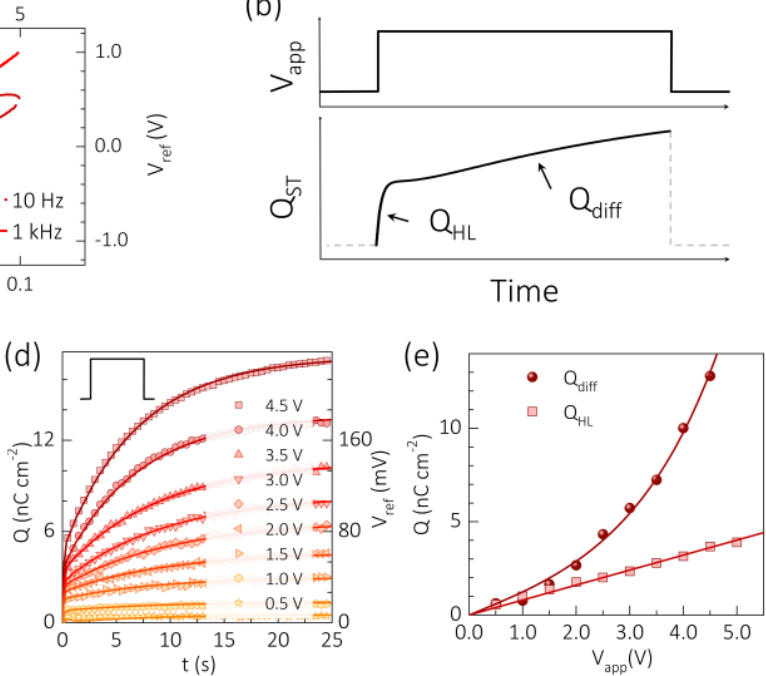

Figure 4.2. Sawyer-Tower circuit characterization of MAPI pellets in dark at room temperature: (a) polarization loops using sinusoidal perturbation, (b) scheme of transient step perturbation and sample signal with two main time constants, transient charging in (c) short and (d) larger time scales, and saturation charge as a function of perturbation amplitude. In (c,b) dots are experimental data. Adapted from ref. ${ }^{[1]}$, Copyright 2016, AIP Publishing LLC.

For MAPI-based PSCs, defects/ions concentrations as large as $10^{17} \mathrm{~cm}^{-3}$ with $L_{D} \sim 10 \mathrm{~nm}$ can be found. ${ }^{[50]}$ Furthermore, analogously to (2.22), the ionic diffusion coefficient can be approached as $D_{\text {ion }}=L_{D}{ }^{2} / \tau_{\text {diff }}$ resulting in the order of $10^{-10} \mathrm{~cm}^{2} \cdot \mathrm{s}^{-1}$ for $\tau_{\text {diff }}$ around 10 seconds.

To sum up, AC characterization of dark capacitance and conductivity spectra, as well as transient experiments under stepped bias, of MAPI pellets were parametrized and associated with different models including electrode polarization, hopping conductivity and double-layer charge accumulation. These considerations suggest that the contribution of ionic phenomena may be of major importance in the performance of MAPI-based optoelectronic devices, particularly PSCs.

\subsection{Dark current-voltage characteristic}

Previous section suggested that ionic phenomena may affect the MAPI conductivity and create charge accumulation at interfaces. Next step would be to investigate complete devices, where the MAPI layer has thicknesses below $1 \mu \mathrm{m}$ and variate morphology features. In addition, the presence of selective contact structure may influence the general optoelectronic response.

In this section, the $J-V$ curves of PSCs in dark are examined as a function of the voltage sweep scan rate $s$, resulting in hysteretic behaviors. Also, the temperature dependency of dark 
$J-V$ hysteresis is discussed. The fabrication and instrumentation details can be found in the original articles. ${ }^{[50,60]}$

\subsubsection{Capacitive dark hysteresis}

The dark logarithm-scaled currents versus the applied voltage are presented in Figure 4.3a, with cycled sweeps at scan rates ranging 1.0 to $50 \mathrm{mV} \mathrm{s}^{-1}$ for the mTmapiClS device (see Table 3.1). For voltages below $0.4 \mathrm{~V}$ the response relies on the direction and speed of the voltage scan sweep, in square-like shapes. The current switches from positive to negative values in the dashed lines regions. One could get dark $J_{s c}$ and $V_{o c}$ values depending on $s$, and even an output power $P_{\text {out }}$ in the forward-to-reverse (FR) scan direction. But those are artifacts of the charging/discharging processes during the voltage sweep.

Above $0.5 \mathrm{~V}$, the hysteretic currents $J_{\text {hyst }}$ are much lower than the operational junction recombination currents $J_{j}$, as (2.26). Then the $J-V$ curves nearly collapse into an average pattern as the voltage increases. This is more evident in the temperature dependent $J-V$ curves of Figure $4.3 \mathrm{~b}$. The overall effect resembles the capacitive square loops of Figure $2.9 \mathrm{c}$, typically reported using electrochemical cyclic voltammetry methods. ${ }^{[61-62]}$

At the slowest scan rate $\left(1.0 \mathrm{mV} \cdot \mathrm{s}^{-1}\right)$, the hysteretic behavior is minimum, and one can approach the average current between sweep directions to $J_{j}$. In the low-voltage range which was studied, $J_{j}$ is significantly sensitive to leakage currents. This is evident from the apparent $V_{o c}$ enhancement at low temperatures in Figure 4.3b. Then, a parametrization as $J_{\text {hyst }}=J-J_{j}$ can be made, and a capacitance versus applied voltage for different scan rates $s$ can be illustrated as $J_{\text {hyst }} /|s|$ in Figure $4.3 \mathrm{~b}$. Nearly linear regions indicate constant capacitance and the inset confirms the linearity $J_{\text {hyst }} \cong C \cdot s$.

The capacitive character of dark hysteresis in low applied voltages can be assumed from its linearity with scan rate, so $J_{\text {hyst }} \cong J_{\text {cap }}$ as equations (2.29) and (2.36). Interestingly, significantly slow scan rates are needed to cancel these capacitive currents. This indicates a retarded kinetics whose nature can be correlated with slow mobile ions. This would agree with the discussions from preceding sections.

The temperature evolution of the capacitance from $J-V$ curves in Figure $4.3 \mathrm{~b}$ is presented and compared with IS measurements in Figure 4.3d. There it is evident that the mechanism behind the dark hysteresis is not significantly affected by the phase transition. This is a device issue, considering the experiments by Stumpp et al. ${ }^{[63]}$ who showed the current from Pt/MAPI/Pt and $\mathrm{Au} / \mathrm{MAPI} / \mathrm{Au}$ samples to step with temperature between 150 and $165 \mathrm{~K}$. Comparing with $10 \mathrm{kHz}$ capacitance from IS, the capacitance from $J-V$ is larger, but as the frequency is reduced larger capacitances are measured by IS. This stress how slower mechanisms take place, increasing capacitance, and hence hysteresis.

Interestingly, note that capacitive features discard the strict "hysteresis" naming for the anomalous currents in the $J-V$ curves of PSCs, in the sense that there is no remnant polarization state. This is additionally checked via ST measurements as in Figure 4.3e. There, only resistor and resistive capacitor behaviors were reported. 

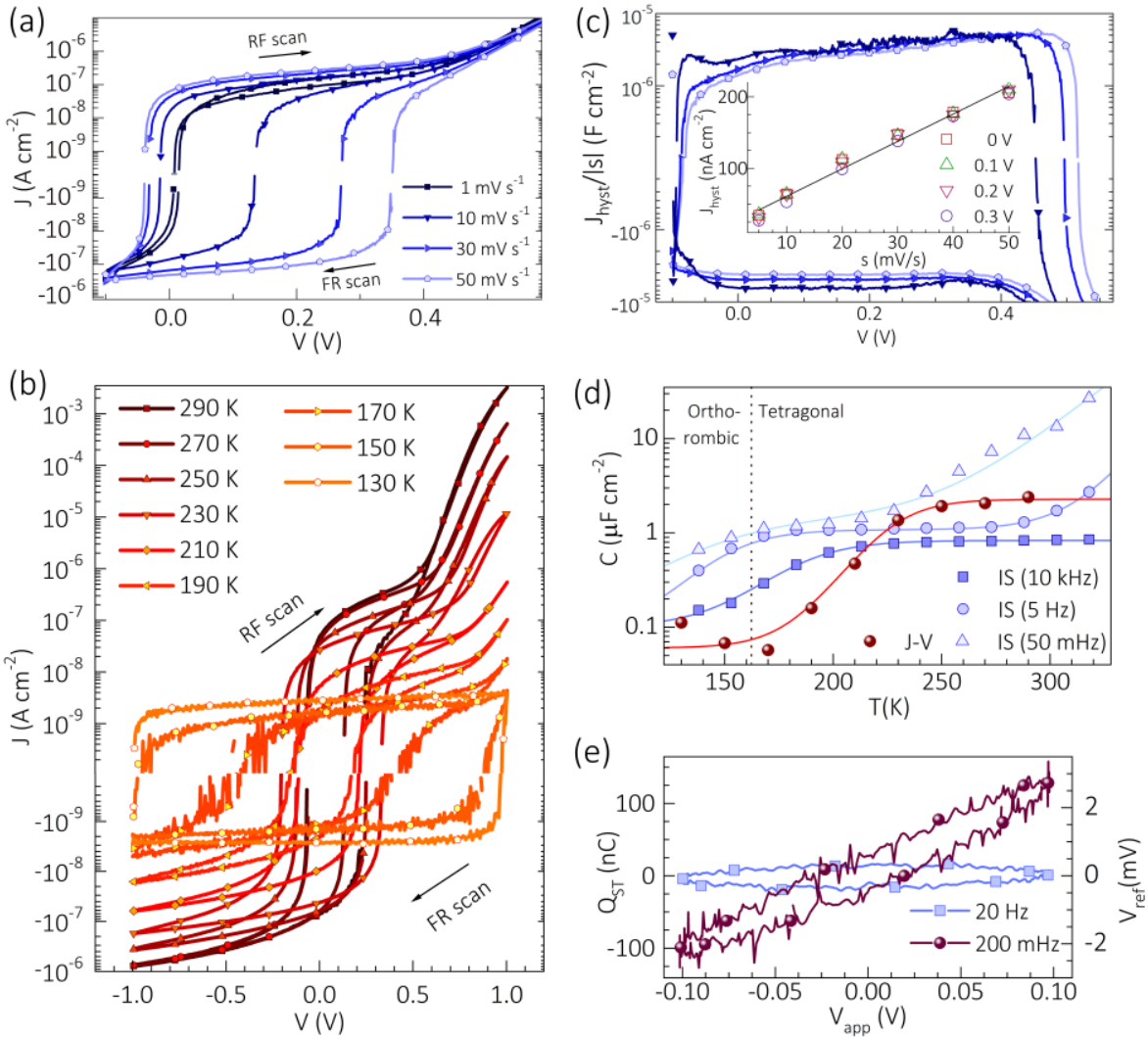

Figure 4.3. Capacitive dark $J-V$ hysteresis of PSCs: $J-V$ curves as a function of (a) scan rate at $300 \mathrm{~K}$, and (b) temperature at $50 \mathrm{mV} \cdot \mathrm{s}^{-1}$, (c) capacitance from (a). Adapted from ref. [50]. Copyright 2015, American Chemical Society. (d) Temperature dependence of capacitance from $J-V$ curves in (b) and IS from Figure 4.5a, and (e) polarization loops from ST setup. Inset in (c) are the capacitive currents (dots) as a function of scan rate for different voltages, as indicated, and the linear fit (line) corresponding to a capacitance of $3.8 \mu \mathrm{F} \cdot \mathrm{cm}^{-2}$.

The effects of capacitive currents in the dark $J-V$ curves of PSCs have been observed in a large variety of materials and device structures (see Table 3.1), beside the most typical $\mathrm{TiO}_{2} / \mathrm{MAPI} /$ spiro array. For instance, by including 2D capping layers (e.g. $\mathrm{AVA}_{2} \mathrm{PbI}_{4}$, $\left.\mathrm{PEA}_{2} \mathrm{PnI}_{4}\right)^{\left[11,{ }^{64-65]}\right.}$ towards the selective contacts and/or changing the composition of the perovskite (e.g. $\mathrm{Cs}_{0.1} \mathrm{FA}_{0.74} \mathrm{MA}_{0.13} \mathrm{PbI}_{2.48} \mathrm{Br}_{0.39}, \mathrm{FA}_{0.83} \mathrm{MA}_{0.17} \mathrm{~Pb}_{1.1} \mathrm{Br}_{0.22} \mathrm{I}_{2.98}$ ), ${ }^{[11,64,66]}$ in general the capacitive hysteresis of well performing devices follows the pattern in Figure 4.3a, with nearly the same capacitance. Interestingly, including self-assembled monolayers of different fullerene derivatives ${ }^{[67]}$ as an interlayer between MAPI and $\mathrm{TiO}_{2}$ in less efficient cells reports similar capacitances but in horizontally shrunken patterns due to the decrease of shunt resistance. On the 
other hand, the time scale for observing the capacitive currents can be increased as the resistivity does for devices using organic materials as selective contacts, e.g. PEDOT:PSS/MAPI/PC ${ }_{70} \mathrm{BM}$ devices, as discussed in the next section.

\subsubsection{Non-capacitive dark hysteresis}

The previous section presented capacitive $J-V$ hysteresis in PSCs. This type of hysteresis may involve capacitive currents in the form $J_{\text {hyst }} \cong C \cdot s$, which modify the operating junction current $J_{j}$ of the cells. Larger hysteresis as the scan rate rises is a well-documented behavior in PSCs. ${ }^{[50,55,57,60,67-72]}$ Moreover, capacitive hysteresis has also been reported in silicon solar cells with large areas at very fast scan rates ${ }^{[73-74]}$ and in CdTe thin film solar cells too. ${ }^{[75]}$ However, as well the opposite trend in PSCs has been reported, i.e. larger hysteresis as the scan rate is decreased ${ }^{[60,76-80]}$ These latter works studied a short range of scan rates under different condition. Thus, a systematic study would be needed trying to cover the different hysteretic phenomena to understand their underlying physical mechanisms.

"Hysteresis-free" behavior in PSCs have been claimed by several authors. ${ }^{[11,66,81-88]}$ Most typically, this is the case of devices with inverted structure (see Figure 2.3b) where organic selective contacts comprise the light harvesting perovskite. ${ }^{[60,88-90]}$ The dark $J-V$ curve measurements at different scan rates for InvMapi samples (see Table 3.1) are illustrated in Figure 4.4a. There, the square-like capacitive loops (see Figure 2.9 and Figure 4.3a) are not evident, because a low capacitance or large leakage currents masking the representation. Anyway, no clear trend of the hysteretic capacitive currents as a function of scan rate is found at short-circuit.

Assuming mobile ions, ${ }^{[50]}$ normal and inverted PSCs with and without large dark capacitive hysteresis, respectively, may indicate two possible scenarios. First, interface features makes the organic selective contacts in the InvMapi cells to inhibit the double-layer charge structures, possibly due to work-function mismatches. ${ }^{[66]}$ Second, lower bulk ionic conductivity due to the change of the complete fabrication conditions when changing $\mathrm{TiO}_{2}$ and spiro-OMeTAD for the PCBM and PEDOT.

A non-capacitive feature arises when slow voltage sweeps around $1.0 \mathrm{mV} \cdot \mathrm{s}^{-1}$ are performed, as in Figure 4.4a. This is a different hysteretic distortion in the dark $J-V$ curves which can be observed around $0.5 \mathrm{~V}$ at forward bias. Note that this extra hysteresis is also present for the normal structure in Figure 4.3a, only that in a lower degree. Summarizing, the simulation of Figure 4.4b illustrates the linear dependence of capacitive currents with scan rates (black solid lines), and the occurrence of non-capacitive distortions at the lowest voltage sweep speeds (red dotted lines).

The experimental capacitive and non-capacitive hysteretic currents of several structures (see Table 3.1), and at different bias conditions are presented in Figure 4.4c. As general empirical trends, $J_{\text {hyst }}$ grows linearly with scan rates when NHyst. Note that this is not a feature only related with low performance devices (10-15\% efficiency) with $\mathrm{TiO}_{2} / \mathrm{MAPI} /$ spiro structure, as originally reported. ${ }^{[50,60,67,91-92]}$ The same capacitive currents are reported for state-of-the-art devices including $2 \mathrm{D}$ capping layers (e.g. $\left.\mathrm{AVA}_{2} \mathrm{PbI}_{4}, \mathrm{PEA}_{2} \mathrm{PnI}_{4}\right)^{[11,64-65]}$ towards the selective contacts with MAPI or $\mathrm{Cs}_{0.1} \mathrm{FA}_{0.74} \mathrm{MA}_{0.13} \mathrm{PbI}_{2.48} \mathrm{Br}_{0.39}$ as perovskite absorbers, ${ }^{[11,64,66]}$ with efficiencies in the range $18-20 \%$. The general trend for non-capacitive currents seems to agree with $J_{\text {hyst }} \propto$ $1 / \sqrt{s}$ and several structures where there is IHyst. However, care must be taken with the 
"universality" of this latter result. IHyst is a more complex phenomena with various origins and reproducibility issues. Thus, one could postulate a general form $J_{\text {hyst }} \propto s^{-r}$, being $r>0$. The total behavior of hysteretic currents is schemed in Figure $4.4 \mathrm{~d}$ highlighting three main regions in terms of the voltage sweep scan rate.

First, at low enough $s$, hysteresis is mainly due to non-capacitive currents $J_{\text {hyst }} \rightarrow J_{\text {noncap }}$ which appear for most of PSCs with an inverse proportionality to the scan rate. Second, at intermediate $s$, hysteretic capacitive currents behave as $J_{\text {hyst }} \rightarrow J_{\text {cap }} \cong C \cdot s$, most typically in PSCs with regular structures. And third, at high enough $s$, the capacitive currents are limited by the capacitor response time constant and hysteretic currents fade. This is also the case when the fast scan rates give no time to the space charge regions to be modified, in terms of slow mobile ion kinetics.

\section{(a)}

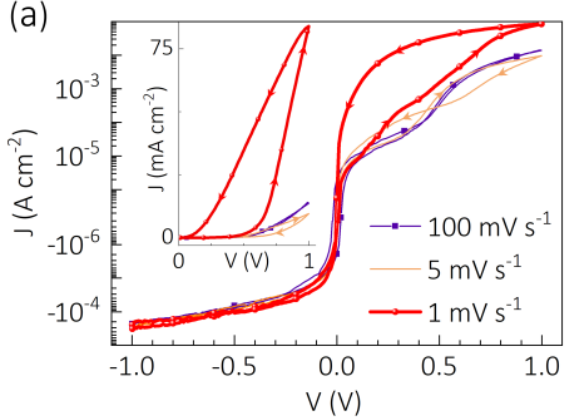

(b)

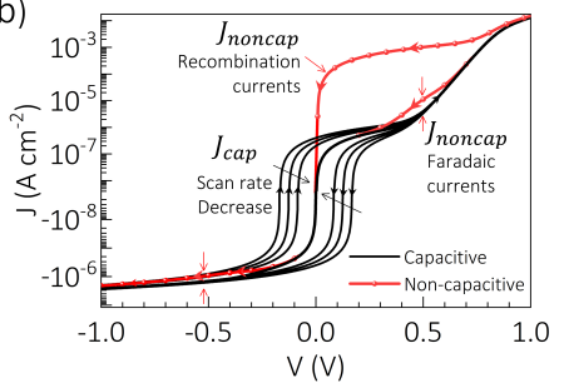

(C)

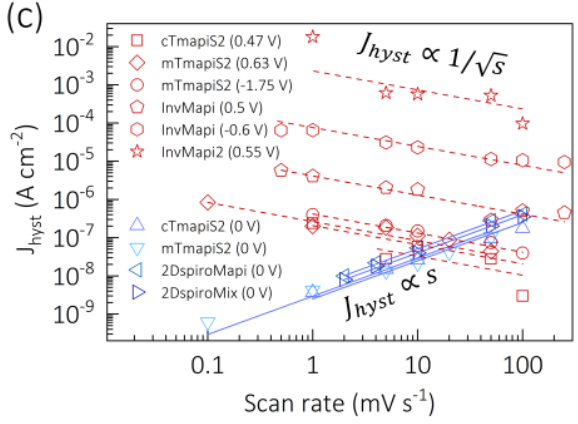

(d)

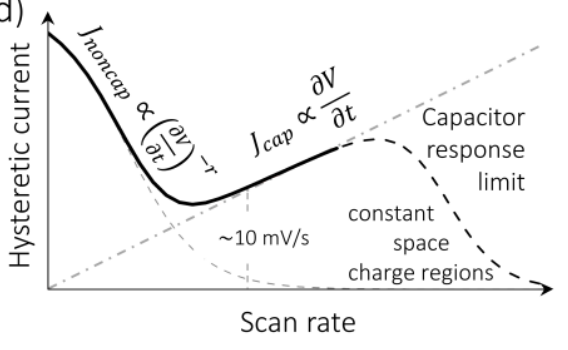

(e)

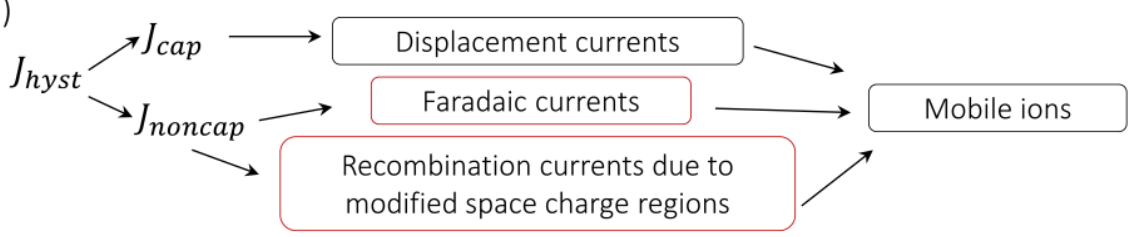

Figure 4.4. Non-capacitive dark $J-V$ hysteresis of PSCs: (a) dark $J-V$ curves as a function of scan rates at room temperature, (b) simulated phenomenology of capacitive and non-capacitive patterns in the dark $J-V$ hysteresis, (c) hysteretic currents from several devices (see Table 3.1) in a broad range of scan rates, and (d) schemed general behavior of hysteretic currents with scan rate showing three main regions. Inset in (a) are the linear 
plots of the same curves. Adapted from ref. [60], Copyright 2016, American Chemical Society. (e) Schemed total contributions to hysteretic currents, connected to the mobile ions.

The nature of the non-capacitive hysteretic currents can be faradaic and/or recombination currents due to modified space-charge regions (see Figure 4.4e). In common, these two mechanisms are favored as the bias is applied for longer times, so $J_{\text {noncap }}$ appear at the slowest scan rates. It has been shown ${ }^{[60]}$ that the reactivity hypothesis fits currents below units of $\mathrm{mA}$ $\mathrm{cm}^{-2}$ behaving as the Nernst-Monod model ${ }^{[93]}$

$$
J_{\text {noncap }}=J_{N M}\left(1+\exp \left[-\frac{q\left(V-V_{N M}\right)}{m_{N M} k_{B} T}\right]\right)^{-1}
$$

which is a step-like law attaining $J_{N M}$ for positive voltages above the characteristic voltage onset $V_{N M}$, related to a reaction potentials. Here $m_{N M}$ is an empirical parameter correcting the effective voltage contributing to the reaction.

These small currents described by (4.8) match capacitance variations related with reversible processes in sort of pseudo-capacitance mechanisms. ${ }^{[60]}$ However, larger $J_{\text {noncap }}$ like in Figure $4.4 \mathrm{a}$ are not so easily explained by reversible Faradaic phenomena.

Space-charges can create larger $J_{\text {noncap }}$ due to the dynamic evolution of drifted ions contributing to the current, while modifying the energy diagram, and hence the electronic recombination currents. If no mobile ions, the junction in equilibrium may produce a time independent operational current $J_{j}(V)$. However, when applying some voltage, independently to the large capacitance and charging/discharging processes, the junction itself is modified and $J_{j}(V, t)$ is different depending on the polarization history.

In closing, it is suggested that dark hysteresis in PSCs results from several phenomena including capacitive, faradaic and space-charge related natures. In all the cases, it looks that ionic transport is of major importance in the electrical performance of PSCs. The scheme in Figure 4.4d seems to be an "universal" map for dark hysteretic currents for practically all of the perovskitebased solar cells, independently of electrodes and/or absorber materials and structures. In the experience of the author, capacitive and/or non-capacitive hysteretic currents can at most be masked by significant resistive artifacts which increase/decrease the time scale of the charge carrier phenomena. But these effects can be understood as horizontal shifts and/or shrinks in the scan rate axis of Figure 4.4d.

\subsection{Dark capacitance from impedance spectroscopy}

Large capacitive currents from the cycled $J-V$ curves at different scan rates were discussed in the previous sections to be an important component of the hysteresis in PSCs. Specifically, evidence suggest that very slow processes influence the overall devices performance, most likely related with ionic phenomena. However, slow processes could be also associated with electronic trapping/detrapping processes due to near-midgap trap states, which would be accessible by IS studies changing temperature and DC bias, i.e. thermal admittance spectroscopy (TAS) and MottSchottky (MS) analysis (see sections 2.6.1-2, 3.3.2-3). 
In this section, TAS and MS analyses in PSCs are discussed. Several limitations and physical inconsistencies are found regarding the low frequency range, suggesting that the ionic phenomena overlap with the possibly electronic signal contribution. This hinders the use of these characterization methods, as discussed in detail in the original works. ${ }^{[50,94-96]}$

\subsubsection{Thermal admittance spectroscopy}

In the TAS approach, temperature dependent steps in the capacitance spectra from IS are assumed due to the emission rate of trap states in the semiconductor bandgap. Most of the reported studies in PSCs use TAS as a comparison method, analyzing the complete frequency rage, presenting DOS curves without clear peaks and showing nearly unrealistically large values. ${ }^{\text {[96-103] }}$

The shape of the capacitance spectra of PSCs usually shows two main characteristics distinguishable at higher and lower frequencies, respectively, as in the Bode plots of Figure 4.5a. In the frequency range above $1 \mathrm{kHz}$, the geometrical capacitance $C_{g}$ and/or the depletion layer capacitance $C_{d l}$ are responsible for the observed plateaus in the spectra. Above $100 \mathrm{kHz}$, the capacitance abruptly drops by effect of the series resistance $R_{s}$. On the other hand, below $10 \mathrm{~Hz}$ the capacitance spectra increase significantly showing a step $C_{s c i}$. From the analysis of previous sections, one could speculate that $C_{s c i}$ relates to mobile ionic space charge regions.

As temperature decrease, the capacitance spectra are left-shifted (towards lower frequencies) and below $200 \mathrm{~K}$ an abrupt down-shifting (towards lower capacitances) occurs. The high frequency general trend is illustrated in terms of $\varepsilon$ in Figure 4.1d, comparing the mTMApiClS and InvMAPI devices with the MAPI pellet sample (see Table 3.1). Unlike the MAPI material, the devices show $\partial \varepsilon / \partial T>0$ and step differently in magnitude and temperature with respect to the phase transition. This could suggest that the abrupt decrease of capacitance at the lower temperatures would depend on the contacts.

Comparing with capacitance from the dark $J-V$ curves, Figure $4.3 \mathrm{~d}$ shows that IS capacitances at the lower frequencies always exceed the capacitance from hysteretic currents. Contrary, around room temperature hysteresis capacitance seems to be larger than that at higher frequencies. This may reinforce the idea that capacitive hysteresis in PSCs is mainly related with the low frequency component of the capacitance spectra.

Increasing the perovskite thickness, the capacitance of cTMapiClS devices (see Table 3.1) is diminished at higher frequencies, as in Figure $4.5 \mathrm{~b}$. Here, a proportionality with $C_{g}$ can be assumed. On the other hand, at lower frequencies a reduction of $C_{s c i}$, when present, is significantly lower. This agrees with the idea of different mechanisms behind the capacitance at low and higher frequencies and suggest that electronic chemical capacitance may not be straightforwardly an explanation for $C_{s c i}$.

Applying the TAS formalism, Equation (3.5) results in the hypothetical DOS depicted in Figure $4.5 \mathrm{c}, \mathrm{d}$. There, one can observe two main peaks. For the higher frequencies (lower $\left.E_{\omega}\right)$ the DOS spectra appear nearly $T$-independent. This may correspond to the coupling $R_{S} C_{g}$, as pointed in Figure 4.5c. The effects of defect densities should be identified in a proper frequency range. For instance, some authors have successfully analyzed TAS in PSCs above $1 \mathrm{kHz}$ but still below the series resistance masking effect around $1 \mathrm{MHz} .^{[104-107]}$ 

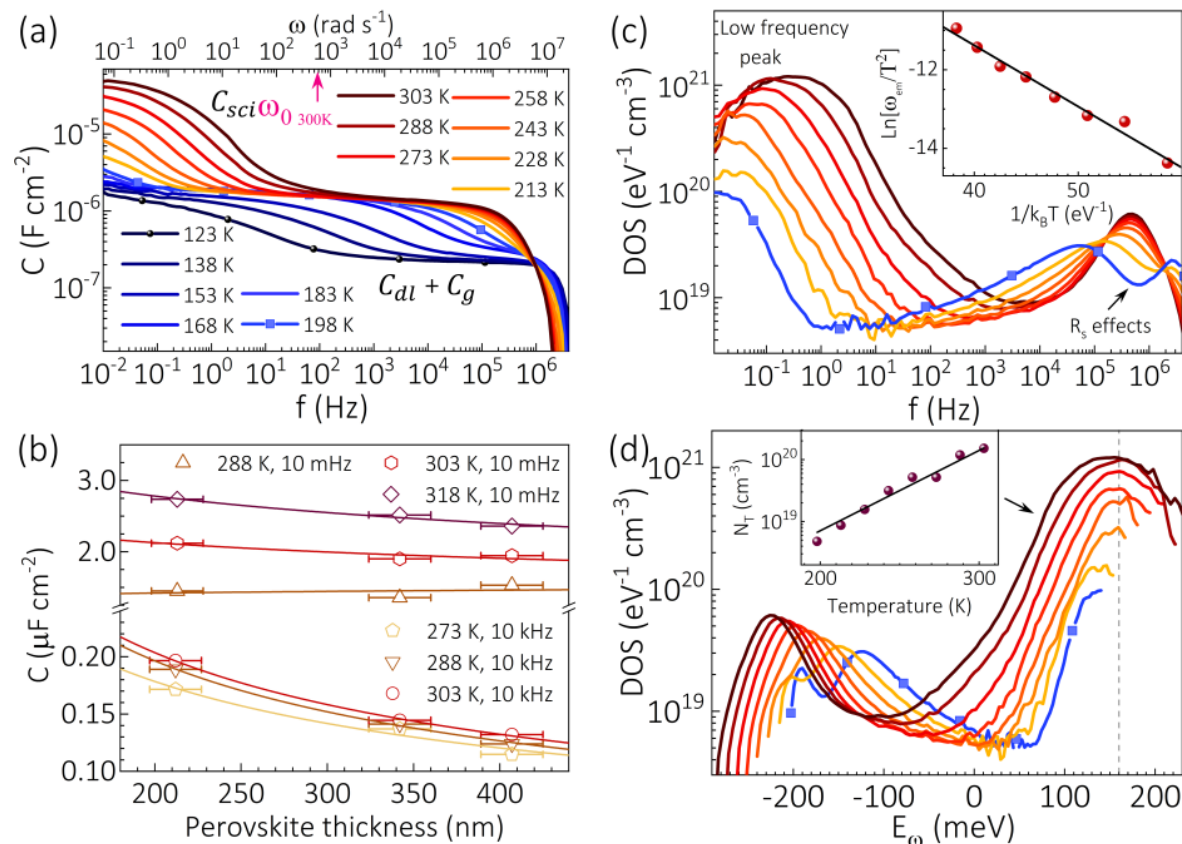

(e)

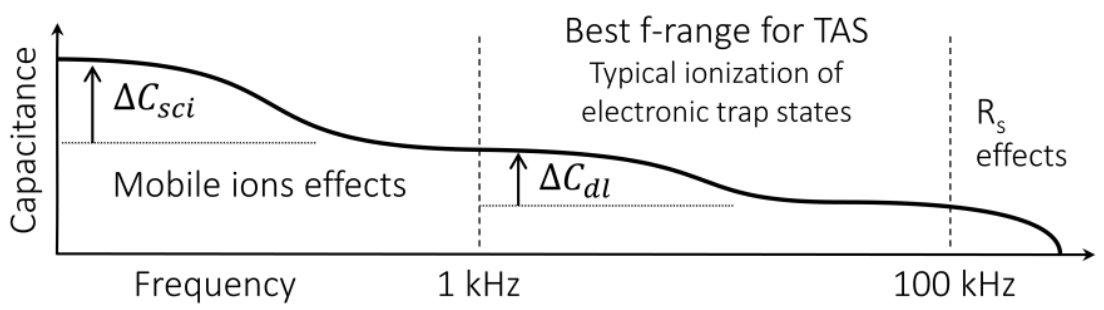

Figure 4.5. Dark capacitance of PSCs via IS at zero DC bias: (a) capacitance versus temperature, (b) capacitance dependence with perovskite thickness and temperature at given frequencies, and DOS as a function of (c) frequency and (d) demarcation energy in the TAS formalism. The DOS is based on Equation (3.5), assuming $V_{b i}=1.0 \mathrm{~V}$ and a perovskite thickness of $200 \mathrm{~nm}$. Inset are (c) the activation energy Arrhenius plot and (d) the trap density versus temperature. Adapted from ref. ${ }^{[50,}{ }^{96]}$, Copyright 2015, 2019, American Chemical Society. (e) Schemed capacitance spectra with different effects according to the frequency ranges.

At the lowest frequencies (highest $E_{\omega}$ ), the large low-frequency capacitance of Figure 4.5a imply large temperature-activated peaks in the DOS spectra. This is illustrated in Figure 4.5c,d where the DOS values can reach $10^{21} \mathrm{eV}^{-1} \mathrm{~cm}^{-3}$ around $E_{\omega} \approx E_{t} \approx 160 \mathrm{meV}$. However, these apparent values for the density of states are hardly linked to the response of any defect levels, considering the nearly intrinsic character of perovskites, having at most doping concentrations around $10^{17}-10^{18} \mathrm{~cm}^{-3} \cdot{ }^{[90,94,96]}$ If one integrates the DOS spectra using Equation (3.6) it would 
result in total defect densities up to $N_{t} \sim 10^{20} \mathrm{~cm}^{-3}$ at room temperature, which appears an entirely unphysical value. Also, the capture cross section $\sigma_{c s} \approx 10^{-24} \mathrm{~cm}^{2}$ and attempt-to-scape frequency at room temperature $\omega_{0}(300 \mathrm{~K}) \approx 527 \mathrm{rad} \cdot \mathrm{s}^{-1}$ (arrow-pointed in Figure 4.5a) have significantly small values. The latter generates negative $E_{\omega}$ values in Figure $4.5 \mathrm{~d}$, which is another unrealistic outcome, due to the low intercept in the Arrhenius plot (inset Figure 4.5c). ${ }^{[108-}$ ${ }^{109]}$ More typical values for these parameters in traditional semiconductors are reported as $\sigma_{c s}>$ $10^{-17} \mathrm{~cm}^{2}$ and $\omega_{0}(300 \mathrm{~K})>10^{6} \mathrm{rad} \cdot \mathrm{s}^{-1} \cdot{ }^{[109-112]}$

The height of the apparent DOS peaks shrink with temperature, as illustrated in Figure $4.5 \mathrm{c}, \mathrm{d}$. This is another anomalous behavior: for trap states one would expect a frequency shift with temperature but with similar amplitudes for the DOS peaks. After the integration, using Equation (3.6), the resulting trap densities exponentially decrease as $N_{t} \approx N_{s 0} \exp \left[T / T_{s 0}\right]$ with $N_{s 0} \approx$ $2 \times 10^{16} \mathrm{~cm}^{-3}$ and $T_{s 0} \approx 34 \mathrm{~K}$ (see inset of Figure $4.5 \mathrm{~d}$ ). This thermally activated process is more likely related to the electrode polarization mechanism discussed in Section 4.1.1 for MAPI pellets, where the conductivity implies ionic-electronic phenomena.

Interestingly, concerning the small values of $\sigma_{c s}$ from TAS in PSCs, it would be interesting to check the Meyer-Neldel rule; i.e. $\sigma_{c s}=\sigma_{c s 0} \exp \left[E_{t} / k_{B} T_{t}\right]$ in Equation (2.49). ${ }^{[13-115]}$ For instance, if the Meyer-Neldel rule would be satisfied, then the activation energy $E_{t}$ from the lowfrequency capacitance $C_{s c i}$ could be the enthalpy increase $\Delta H$ for the thermodynamic system upon ionization at a given temperature. Then, the consequent entropy change would be $\Delta S=E_{t} / T_{t}$.

Importantly, the contact layers can add extra masking and/or competing capacitance contributions, besides the property of perovskite absorbers of showing slow mechanisms evidenced in the low frequency part of the capacitance spectra and most likely of ionic nature. For instance, it is well known that the dark low frequency capacitance of devices with organic selective contacts (see Table 3.1 for PEDOT:PSS/MAPI/PC 70 BM or $\mathrm{C}_{60}$-SAM/MAPI/PDCBT) ${ }^{[60}$, $66,95]$ is typically lower than that for cells with normal structure $\mathrm{TiO}_{2} / \mathrm{MAPI} /$ spiro. In some cases it can be nearly flat. ${ }^{[16]}$ This is first because of resistive artifacts which can shift the low frequency step towards lower frequencies. ${ }^{[95]}$ On the other hand, being the dark low-frequency capacitance an interphase phenomena, as suggested from the characterization of gold contacted MAPI pellets in Section 4.1, ${ }^{[1]}$ it makes sense that selective layers are of major importance on the charge accumulation towards the interfaces. Purposely, R.A. Awni et al. ${ }^{[17]}$ measured TAS in samples with various configurations $\left(\mathrm{SnO}_{2} / \mathrm{C}_{60}-\mathrm{SAM} / \mathrm{MA}_{0.7} \mathrm{FA}_{0.3} \mathrm{PbI}_{3} /\right.$ spiro, $\mathrm{SnO}_{2} / \mathrm{C}_{60}-\mathrm{SAM} /$ spiroOMeTAD and $\mathrm{SnO}_{2} / \mathrm{C}_{60}-\mathrm{SAM} / \mathrm{MA}_{0.7} \mathrm{FA}_{0.3} \mathrm{PbI}_{3}$ ) and showed that the thermally activated capacitance in the range $120 \mathrm{~K}-300 \mathrm{~K}$ and below $10 \mathrm{kHz}$ are actually mainly due to the hole transport layer, i.e. the spiro-OMeTAD.

In summary, the application of the TAS method in PSCs should be limited to the range from $1 \mathrm{kHz}$ to $100 \mathrm{kHz}$ discarding masking/overlapping effects of $C_{s c i}$ and $R_{S}$, towards lower and higher frequencies, respectively (see Figure 4.5e). At frequencies below $1 \mathrm{kHz}$ the capacitance spectra increase due to slow dynamic processes, hardly connected with DOS and most likely to ionic phenomena. Furthermore, the capacitive effects of the selective contacts should also be considered as extra sources of masking or contributing signal. 


\subsubsection{Mott-Schottky analyses}

Low frequency dark capacitance spectra with thermally activated ionic processes at zero DC bias were discussed in the previous section. Alternatively, one can characterize the capacitance at a single high frequency (e.g. $10 \mathrm{kHz}$ ) varying the DC voltage at room temperature, which is the case of the MS analyses.

Two general cases of MS patterns have been reported for PSCs. First, a dependence $C^{-2} \propto V$ is noticeable from quasi-equilibrium towards forward bias in Figure 4.6a, which obeys Equation (2.46) and allows extracting the built-in voltage $V_{b i}$ from the intercept of the linear fitting and the doping concentration $N$ from the corresponding slope. Towards reverse bias, the capacitance collapses to $C_{g}$ because of the full depletion regime. Contrary, if the applied voltage approaches and/or exceeds $V_{b i}$ at forward bias, the capacitance grows exponentially, as in the right axis in Figure 4.6a. These are relatively standard behaviors, but anyway note that validating the MS analysis requires the linear trend $C^{-2} \propto V$ in the range between zero bias and low forward bias as a necessary condition, despite it is not a sufficient condition. ${ }^{[90,94,118]}$ Potentially valid MS analysis in PSCs are scarcely reported. ${ }^{[119-120]}$ Figure 4.6a illustrates the behavior of $\mathrm{TiO}_{2} / \mathrm{CH}_{3} \mathrm{NH}_{3} \mathrm{PbI}_{3-\mathrm{x}} \mathrm{Cl}_{\mathrm{x}} /$ spiro devices whose calculated defect density was $N \approx 10^{17} \mathrm{~cm}^{-3}$. [94] However, this was the only cell configuration which exhibited a doping-like behavior among all the studied samples as listed in Table 3.1. ${ }^{[50,121]}$ Furthermore, the pattern of Figure 4.6a is only habitual among $\mathrm{ASnX}_{3}$ perovskite based devices. ${ }^{[122-124]}$

Most commonly, a second MS plot type like Figure 4.6b can be obtained from PSCs, where the MS analysis is unpractical. ${ }^{[94]}$ At reverse bias, the capacitance seems saturated, and these nearly constant values are not so different even at low forward biases. If one would try to make a linear fitting in the range $0.0-0.4 \mathrm{~V}$, the resulting $N$ and $V_{b i}$ values would be unrealistically large, e.g. $N \approx 10^{19} \mathrm{~cm}^{-3}$ and $V_{b i} \approx 10 \mathrm{~V}$ in Figure $4.6 \mathrm{~b}$. This "fingerprint" allows to rule out the patterns like Figure $4.6 \mathrm{~b}$ for applying the MS analysis.

Incorrectly, one would be tempted to fit the apparently linear section ranging $0.4 \mathrm{~V}$ to $0.8 \mathrm{~V}$ in Figure 4.6b. But this is not $C_{d l}$. Instead, it is the effect of the exponentially increasing capacitance, evident in the right axis of Figure $4.6 \mathrm{~b}$.

The MS pattern of Figure 4.6b is not an unique response of PSCs, but the signature of p-i-n junctions where the depleted region width $w_{n}+w_{p}$ in the selective contacts is much smaller that the intrinsic width $w_{i}$ in the active layer (see Figure 2.10). This is typically the case in organic solar cells. ${ }^{[125]}$ Then $\left(w_{n}+w_{p}\right) \ll w_{i}$ implies that the total depletion layer width $w$ is nearly unchanged $w \approx w_{i}$. For instance, in an OSA p-i-n junction the MS analysis would result

$$
C^{-2}=\left(\frac{w_{i}}{\varepsilon_{0} \varepsilon}+\sqrt{\frac{2}{q \varepsilon_{0} \varepsilon N}\left(V_{b i}-V\right)}\right)^{2}
$$

Note that (4.9) fits better the slight MS plot curvature as in Figure 4.6b below $0.4 \mathrm{~V}$. Nevertheless, for flatter patterns $w^{2}=w_{i}{ }^{2}+w_{p}{ }^{2}+w_{n}{ }^{2}$ can be more suitable (see Table 2.1). [126-127]

A sort of MS plot hysteresis in PSCs, like the experimental curves of Figure 4.6c, occurs if the DC bias is cycled at different scan rates, similarly to the $J-V$ hysteresis. This suggest that 
one can tune the charge density distribution $\rho(x)$, and thus the MS plot, depending on the polarization history and the effects are evident even at frequencies as high as $10 \mathrm{kHz}$.
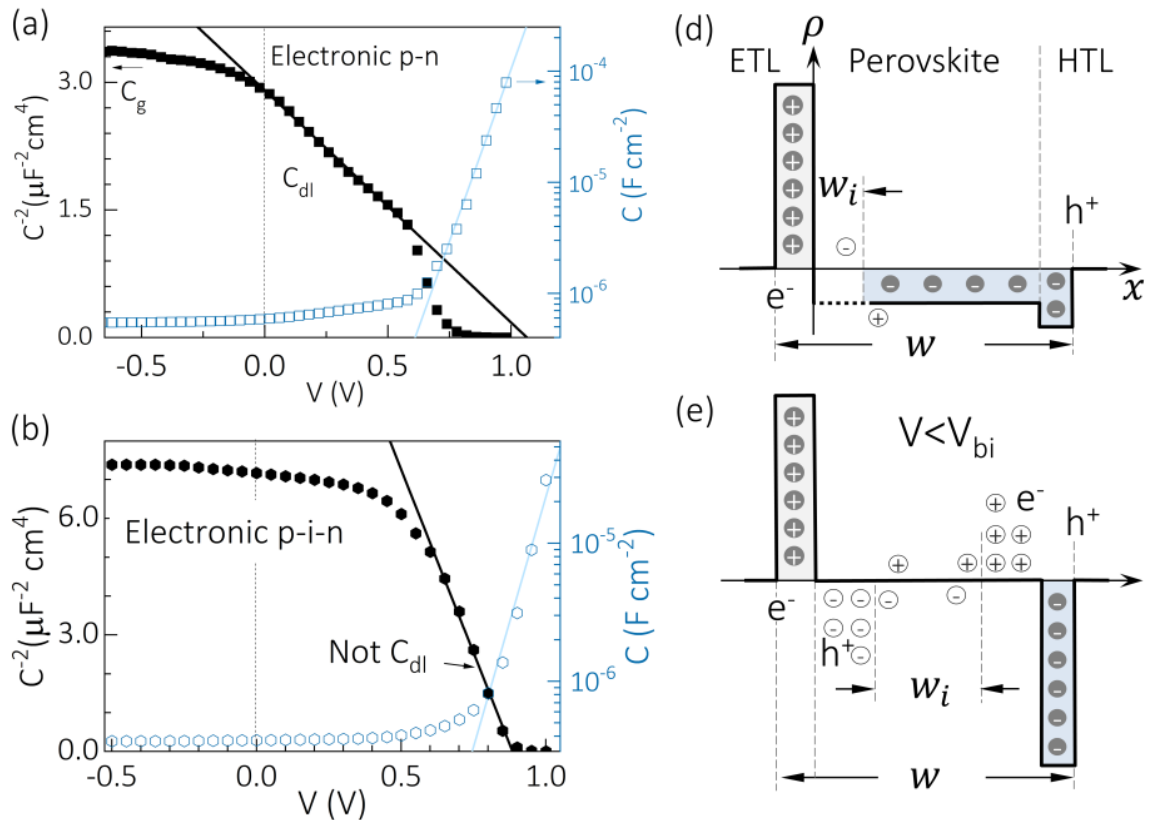

(e)
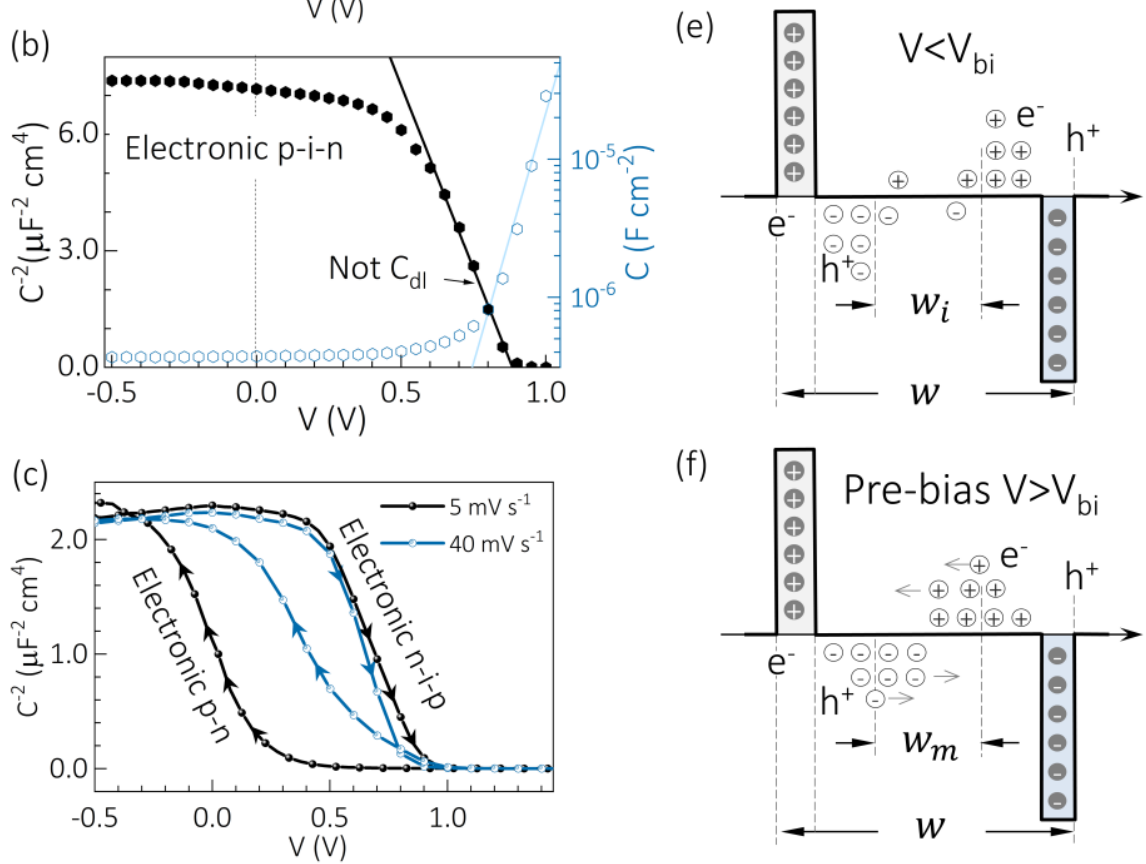

(f)

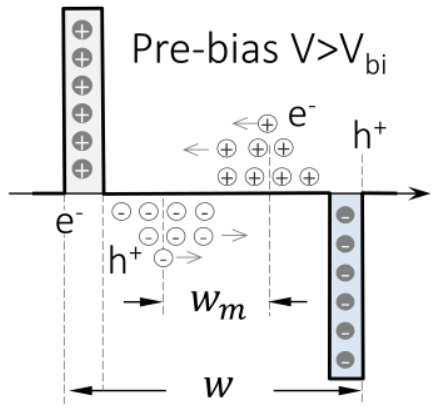

Figure 4.6. Mott-Schottky analysis of PSCs at $10 \mathrm{kHz}$ : MS plots (left axis) and capacitance voltage (right axis) from devices including (a) $\mathrm{CH}_{3} \mathrm{NH}_{3} \mathrm{PbI}_{3-\mathrm{x}} \mathrm{Cl}_{\mathrm{x}}$ and (b) $\mathrm{CH}_{3} \mathrm{NH}_{3} \mathrm{PbI}_{3}$ as absorbers. Adapted from ref. ${ }^{[94]}$, Copyright 2016, AIP Publishing. (c) MS plots as a function of DC bias sweep scan rate (MAPI cell), and (d-f) corresponding charge density profiles. Filled and open circles symbol fixed doping depleted ions and mobile ions, respectively, and $\mathrm{e}^{-}$and $\mathrm{h}^{+}$indicate electrons and holes, respectively.

The different charge density profiles in each situation are illustrated in Figure 4.6d-f. A lightly doped perovskite like Figure 4.6d with/without a small intrinsic region (or a thin intrinsic perovskite layer) could be the case belonging to the p-n junction like behavior of the MS of Figure 
4.6a. Differently, a much larger intrinsic region width is presented in Figure 4.6e, which may correspond to the p-i-n junction like behavior of Figure 4.6b.

The presence of slow mobile ions in the perovskites is an already accepted statement, given the several experimental and theoretical studies in agreement. ${ }^{[20,30,128]}$ Particularly, the slow kinetic under illumination was attributed to MA cation by Senocrate et al., ${ }^{[129-131]}$ while iodine vacancies are considered as the main contribution to faster ionic conductivity.

The distribution of mobile ions (open circles in Figure 4.6d-f) can effectively modify the intrinsic region width. For DC biases below $V_{b i}$, they would be piled up to the interfaces like in Figure 4.6e, where their contribution to the capacitance may be still lower than that of the intrinsic region. However, if larger DC biases are applied, the mobile ions may be drifted to the opposite interface. In this configuration there is no more $w_{i}$, and the electronic charge may respond to AC perturbation like a parallel plate capacitor with distance between plates $w_{m}$.

Purposely, Fischer et al. ${ }^{[132]}$ proposed that the mobile ions could fully invert their equilibrium distribution aligning with the fixed depleted ions and forming a "perfect" $p$-n junction, upon large enough DC forward bias value and polarization time. They showed the transition from p-i-n to p$\mathrm{n}$ like behaviors after $2 \mathrm{~V}$ pre-bias in formamidinium-based PSCs.

The electronic character of MS plots in Figure 4.6 is highlighted because the capacitance measurements were taken at $10 \mathrm{kHz}$. Herein it is assumed that the main ionic effect is that of the modification of $\rho$ through the tuning of $w_{i}$. However, additional evidence can be obtained if the low frequency part of the IS spectra is analyzed, as in Figure 4.7a. The complete spectra as a function of DC bias can be fitted to equivalent circuit (EC) model in Figure 4.7b, most successfully below $0.8 \mathrm{~V}$. In that $\mathrm{EC}$, besides the series resistance $R_{S}$, two resistances and capacitances are labeled as respond to low or high frequencies, $R_{L f}, C_{L f}, R_{H f}$ and $C_{H f}$, respectively.

Comparing the MS plots in terms of frequencies, it evidences that electronic response at 10 $\mathrm{kHz}$ behaves like a p-i-n junction, while ionic $C_{L f}$ from fittings shows a p-n junction like trend, or a much lesser $w_{i} \mathrm{p}$-i-n junction like response, as in Figure 4.7c. The simulations (lines in Figure 4.7c) suggest a good agreement with the above ideas. Note that for the capacitance the $C_{s}$ and $C_{p}$ (as in Table 3.4) are shown in Figure $4.7 \mathrm{c}$ at $10 \mathrm{kHz}$, which are the typical automatic outcomes from commercial work stations for MS analyses.

High injection condition, when DC bias exceeds $V_{b i}$, makes an exponential increase of capacitance where further mechanisms can contribute. This is already evident from the need of a new EC model. Below, $0.8 \mathrm{~V}$ both EC Figure 4.7b,d can fit the experimental spectra, most successfully that of Figure 4.7b,d. However, above $0.8 \mathrm{~V}$ not only Figure $4.7 \mathrm{~d}$ is the most appropriate, but it requires a constant-phase element substituting $C_{L f}$ with power parameter $\theta$ between 0.55 and 0.65 , which is closer to a Warburg diffusion element than to a capacitor. This suggest a closer relationship between the mobile ions distribution and the traditional diffusion capacitance.

In agreement with the above discussions, what we should take for granted is that the MS analysis in PSCs requires "double check" for overlapping effects before any report. ${ }^{[90]}$ Unscrupulous evaluations of $V_{b i}$ and $N$ from MS plots with patterns like Figure 4.6b are examples of bad practices. ${ }^{[133-140]}$ Nevertheless, one can still use the MS representation as a qualitative relative comparison method for checking changes in the apparent $V_{b i}$ value. ${ }^{[66]}$ 

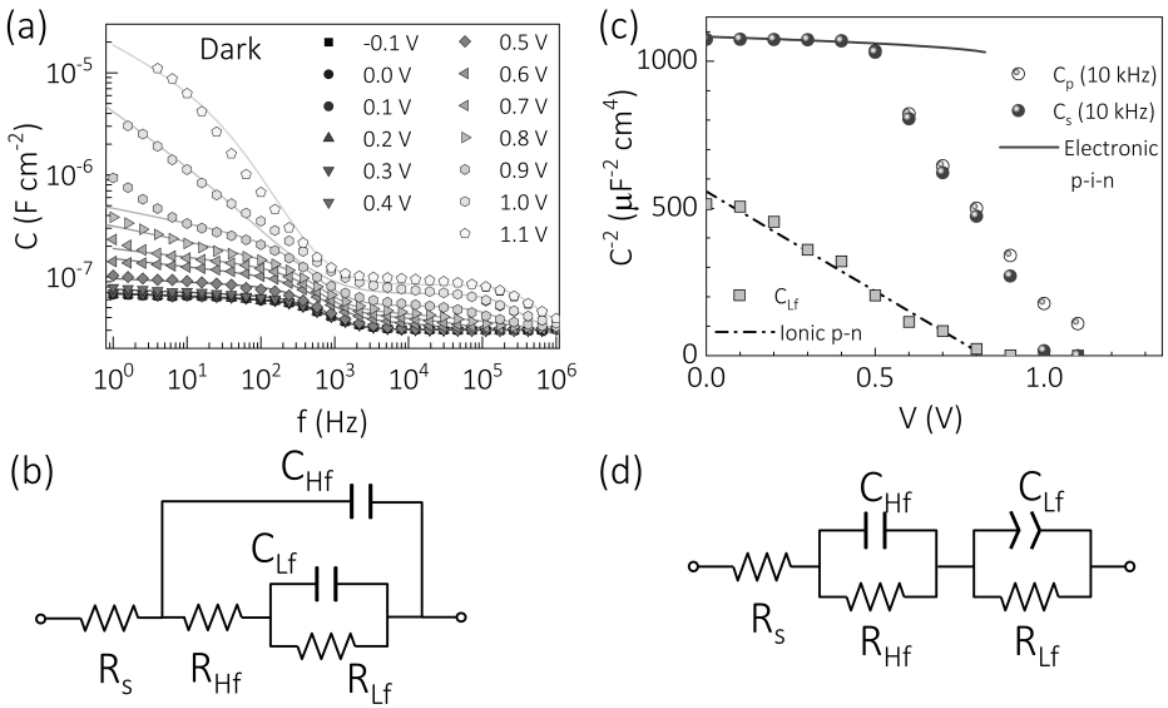

(d)

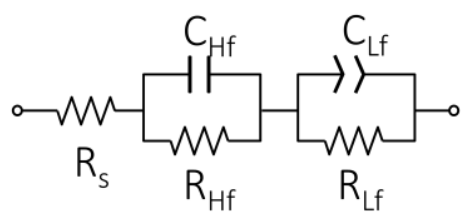

(e)

$$
\begin{gathered}
\text { p-n or } p-i-n \text { junction } \\
\left(w_{i} \ll w\right)
\end{gathered}
$$$$
+ \text { Mobile ions }=
$$

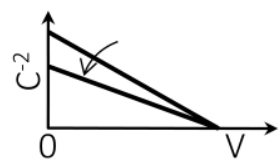

$$
\begin{gathered}
\text { p-i-n junction } \\
\left(w_{i} \sim w\right)
\end{gathered}
$$

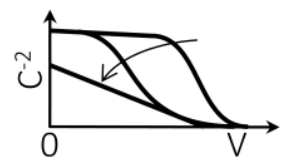

Figure 4.7. Dark capacitance versus DC bias at room temperature of a PPmixP device (see Table 3.1): (a) spectra, (b) Mott-Schottky analysis under different conditions, as indicated, and (c, d) equivalent circuit models for fittings (lines) of data (dots) in (a). Simulations in (c) fitted $C_{L f}$ to (4.9) with $w_{i}=0, N=9.1 \times 10^{15} \mathrm{~cm}^{-3}$ and $V_{b i}=0.83 \mathrm{~V}$ and assumed $w_{i}=707 \mathrm{~nm}, N=5 \times 10^{18} \mathrm{~cm}^{-3}$ and $V_{b i}=0.85 \mathrm{~V}$ for the capacitances at $10 \mathrm{kHz}$. (e) Schemed summary of the mobile ions effects in the MS plots.

In summary, the MS analysis in PSCs is mostly inhibited by the p-i-n junction structure of these devices and, additionally, affected by most likely ionic effects masking the actual depletion layer capacitance. The intrinsic character of most of perovskites with photovoltaic applications is one of the most constant properties reported in these devices, excepting Sn-based perovskite absorbers and some including chloride. Additionally, the ionic-related features hinder the accurate junction characterization and can be easily identified, as summarized in Figure 4.7e. Therefore, the evaluation of the doping densities and the built-in voltage requires additional validation procedures and/or experiments. 


\section{References}

[1] O. Almora, A. Guerrero, G. Garcia-Belmonte, Appl. Phys. Lett. 108 (2016), 043903. https://doi.org/10.1063/1.4941033

[2] F. Brivio, A. B. Walker, A. Walsh, APL Mater. 1 (2013), 042111. https://doi.org/10.1063/1.4824147

[3] F. Brivio, K. T. Butler, A. Walsh, M. van Schilfgaarde, Phys. Rev. B 89 (2014), 155204. https://doi.org/10.1103/PhysRevB.89.155204

[4] J.-S. Park et al., J. Phys. Chem. Lett. 6 (2015), 4304. https://doi.org/10.1021/acs.jpclett.5b01699

[5] D. O. Demchenko et al., Phys. Rev. B 94 (2016), 075206.

https://doi.org/10.1103/PhysRevB.94.075206

[6] M. Sendner et al., Mater. Horiz. 3 (2016), 613. https://doi.org/10.1039/C6MH00275G

[7] M. Shirayama et al., Phys. Rev. Appl. 5 (2016), 014012.

https://doi.org/10.1103/PhysRevApplied.5.014012

[8] J. Zhou, F. L. Tang, H. T. Xue, F. J. Si, Mater. Sci. Forum 850 (2016), 245.

https://doi.org/10.4028/www.scientific.net/MSF.850.245

[9] M. A. Pérez-Osorio et al., J. Phys. Chem. C 121 (2017), 18459.

https://doi.org/10.1021/acs.jpcc.7b07121

[10] P. Umari, E. Mosconi, F. De Angelis, J. Phys. Chem. Lett. 9 (2018), 620.

https://doi.org/10.1021/acs.jpclett.7b03286

[11] O. Almora et al., Sol. Energy Mater. Sol. Cells 195 (2019), 291.

https://doi.org/10.1016/j.solmat.2019.03.003

[12] A. Yang et al., J. Nanomed. Nanotechnol. 7 (2016), 407. https://doi.org/10.4172/2157-7439.1000407

[13] J. A. Guerra et al., J. Appl. Phys. 121 (2017), 173104. https://doi.org/10.1063/1.4982894

[14] P. Löper et al., J. Phys. Chem. Lett. 6 (2015), 66. https://doi.org/10.1021/jz502471h

[15] C. G. Bailey, G. M. Piana, P. G. Lagoudakis, J. Phys. Chem. C 123 (2019), 28795.

https://doi.org/10.1021/acs.jpcc.9b08903

[16] N. Onoda-Yamamuro, T. Matsuo, H. Suga, J. Phys. Chem. Solids 53 (1992), 935.

https://doi.org/10.1016/0022-3697(92)90121-S

[17] A. Mohanty et al., ACS Energy Lett. 4 (2019), 2045. https://doi.org/10.1021/acsenergylett.9b01291

[18] X.-K. Ding et al., Acta Phys.-Chim. Sin. 31 (2015), 576.

https://doi.org/10.3866/PKU.WHXB201501201

[19] D. H. Fabini et al., J. Phys. Chem. Lett. 7 (2016), 376. https://doi.org/10.1021/acs.jpclett.5b02821

[20] T.-Y. Yang et al., Angew. Chem. Int. Ed. 54 (2015), 7905. https://doi.org/10.1002/anie.201500014

[21] A. Slonopas, B. Kaur, P. Norris, Appl. Phys. Lett. 110 (2017), 222905.

https://doi.org/10.1063/1.4984817

[22] A. Slonopas, H. Ryan, P. Norris, Electrochimica Acta 307 (2019), 334.

https://doi.org/10.1016/j.electacta.2019.03.221

[23] J. H. Beaumont, P. W. M. Jacobs, J. Phys. Chem. Solids 28 (1967), 657.

https://doi.org/10.1016/0022-3697(67)90097-2

[24] C. Kim, M. Tomozawa, J. Am. Ceram. Soc. 59 (1976), 127. https://doi.org/10.1111/j.11512916.1976.tb09448.x

[25] M. Samet et al., J. Chem. Phys. 142 (2015), 194703. https://doi.org/10.1063/1.4919877

[26] O. Almora, A. González-Lezcano, A. Guerrero, C. J. Brabec, Phys. Rev. Appl. (2020), submitted.

[27] D. Yang et al., Chem. Mater. 28 (2016), 4349. https://doi.org/10.1021/acs.chemmater.6b01348

[28] B. Gebremichael, G. Alemu, G. Tessema Mola, Phys. B Condensed Mater 514 (2017), 85.

https://doi.org/10.1016/j.physb.2017.03.035

[29] O. Knop et al., Canadian J. Chem. 68 (1990), 412. https://doi.org/10.1139/v90-063

[30] D. Głowienka, T. Miruszewski, J. Szmytkowski, Solid State Sci. 82 (2018), 19.

https://doi.org/10.1016/j.solidstatesciences.2018.05.009

[31] M. S. Sheikh, A. P. Sakhya, A. Dutta, T. P. Sinha, Thin Solid Films 638 (2017), 277.

https://doi.org/10.1016/j.tsf.2017.07.070

[32] M. S. Sheikh et al., Ferroelectrics 514 (2017), 146. https://doi.org/10.1080/00150193.2017.1359023

[33] A. K. Jonscher, Nature 267 (1977), 673. https://doi.org/10.1038/267673a0

[34] E. F. Hairetdinov, N. F. Uvarov, H. K. Patel, S. W. Martin, Phys. Rev. B 50 (1994), 13259.

https://doi.org/10.1103/PhysRevB.50.13259 
[35] R. M. Hill, A. K. Jonscher, J. Non-Crystalline Solids 32 (1979), 53. https://doi.org/10.1016/00223093(79)90064-4

[36] J. C. Dyre, T. B. Schrøder, Rev. Mod. Phys. 72 (2000), 873.

https://doi.org/10.1103/RevModPhys.72.873

[37] S. R. Elliott, Solid State Ionics 70-71 (1994), 27. https://doi.org/10.1016/0167-2738(94)90284-4

[38] J. C. Dyre, P. Maass, B. Roling, D. L. Sidebottom, Rep. Prog. Phys. 72 (2009), 046501.

https://doi.org/10.1088/0034-4885/72/4/046501

[39] M. Pollak, G. E. Pike, Phys. Rev. Lett. 28 (1972), 1449. https://doi.org/10.1103/PhysRevLett.28.1449

[40] W. K. Lee, J. F. Liu, A. S. Nowick, Phys. Rev. Lett. 67 (1991), 1559.

https://doi.org/10.1103/PhysRevLett.67.1559

[41] L. Murawski, R. J. Barczyński, D. Samatowicz, Solid State Ionics 157 (2003), 293.

https://doi.org/10.1016/S0167-2738(02)00224-2

[42] A. Mansingh, Bull. Mater. Sci. 2 (1980), 325. https://doi.org/10.1007/BF02908579

[43] S. R. Elliott, A. P. Owens, Philos. Mag. B 60 (1989), 777.

https://doi.org/10.1080/13642818908209742

[44] G. Conte, F. Somma, M. Nikl, Phys. Status Solidi C 2 (2005), 306.

https://doi.org/10.1002/pssc.200460171

[45] G. Vitale, G. Conte, P. Aloe, F. Somma, Mater. Sci. Eng., C 25 (2005), 766.

https://doi.org/10.1016/j.msec.2005.06.016

[46] P. Maji et al., J. Appl. Phys. 124 (2018), 124102. https://doi.org/10.1063/1.5026038

[47] P. Maji, S. Chatterjee, S. Das, Ceram. Int. 45 (2019), 6012.

https://doi.org/10.1016/j.ceramint.2018.12.071

[48] K. Shimakawa, in Encyclopedia of Materials: Science and Technology, (Eds: K. H. J. Buschow, R. W. Cahn, M. C. Flemings, B. Ilschner, E. J. Kramer, S. Mahajan, P. Veyssière), Elsevier, Oxford 2001, 3579. https://doi.org/10.1016/B0-08-043152-6/00637-9

[49] M. Coll et al., J. Phys. Chem. Lett. 6 (2015), 1408. https://doi.org/10.1021/acs.jpclett.5b00502

[50] O. Almora et al., J. Phys. Chem. Lett. 6 (2015), 1645. https://doi.org/10.1021/acs.jpclett.5b00480

[51] A. Slonopas, B. J. Foley, J. J. Choi, M. C. Gupta, J. Appl. Phys. 119 (2016), 074101.

https://doi.org/10.1063/1.4941532

[52] B. E. Conway, in Electrochemical Supercapacitors: Scientific Fundamentals and Technological Applications, (Ed: B. E. Conway), Springer US, Boston, MA 1999, 105. https://doi.org/10.1007/978-14757-3058-6_6

[53] A. González, E. Goikolea, J. A. Barrena, R. Mysyk, Renewable Sustainable Energy Rev. 58 (2016), 1189. https://doi.org/10.1016/j.rser.2015.12.249

[54] L. Zhang et al., Renewable Sustainable Energy Rev. 81 (2018), 1868.

https://doi.org/10.1016/j.rser.2017.05.283

[55] R. S. Sanchez et al., J. Phys. Chem. Lett. 5 (2014), 2357. https://doi.org/10.1021/jz5011187

[56] A. Baumann et al., APL Mater. 2 (2014), 081501. https://doi.org/10.1063/1.4885255

[57] W. Tress et al., Energy Environ. Sci. 8 (2015), 995. https://doi.org/10.1039/C4EE03664F

[58] J. Beilsten-Edmands et al., Appl. Phys. Lett. 106 (2015), 173502. https://doi.org/10.1063/1.4919109

[59] V. S. Bagotsky, in Fundamentals of Electrochemistry, John Wiley \& Sons, Inc., 2005, 705. https://doi.org/10.1002/047174199X.app2

[60] O. Almora et al., ACS Energy Lett. 1 (2016), 209. https://doi.org/10.1021/acsenergylett.6b00116

[61] F. Fabregat-Santiago, I. Mora-Seró, G. Garcia-Belmonte, J. Bisquert, J. Phys. Chem. B 107 (2003), 758. https://doi.org/10.1021/jp0265182

[62] R. Hass, J. García-Cañadas, G. Garcia-Belmonte, J. Electroanal. Chem. 577 (2005), 99. https://doi.org/10.1016/j.jelechem.2004.11.020

[63] M. Stumpp, R. Ruess, J. Müßener, D. Schlettwein, Mater. Today Chem. 4 (2017), 97. https://doi.org/10.1016/j.mtchem.2017.03.001

[64] O. Almora et al., Nano Energy 48 (2018), 63. https://doi.org/10.1016/j.nanoen.2018.03.042

[65] K. T. Cho et al., Energy Environ. Sci. 11 (2018), 952. https://doi.org/10.1039/C7EE03513F

[66] A. Guerrero et al., Adv. Energy Mater. 8 (2018), 201703376.

https://doi.org/10.1002/aenm.201703376

[67] M. Valles-Pelarda et al., J. Phys. Chem. Lett. 7 (2016), 4622.

https://doi.org/10.1021/acs.jpclett.6b02103

[68] A. Dualeh et al., ACS Nano 8 (2014), 362. https://doi.org/10.1021/nn404323g 
[69] E. L. Unger et al., Energy Environ. Sci. 7 (2014), 3690. https://doi.org/10.1039/C4EE02465F

[70] H.-S. Kim, N.-G. Park, J. Phys. Chem. Lett. 5 (2014), 2927. https://doi.org/10.1021/jz501392m

[71] J. A. Christians, J. S. Manser, P. V. Kamat, J. Phys. Chem. Lett. 6 (2015), 852. https://doi.org/10.1021/acs.jpclett.5b00289

[72] B. Chen et al., J. Phys. Chem. Lett. 6 (2015), 4693. https://doi.org/10.1021/acs.jpclett.5b02229

[73] M. Herman, M. Jankovec, M. Topič, Int. J. Photoenergy 2012 (2012), 11. https://doi.org/10.1155/2012/151452

[74] G. Friesen, H. A. Ossenbrink, Sol. Energy Mater. Sol. Cells 48 (1997), 77. https://doi.org/10.1016/S0927-0248(97)00072-X

[75] D. S. Albin, J. A. del Cueto, "Effect of Hysteresis on Measurements of Thin-Film Cell Performance", presented at SPIE Solar Energy + Technology, San Diego, California, USA, 2010. https://doi.org/10.1117/12.861175

[76] H. J. Snaith et al., J. Phys. Chem. Lett. 5 (2014), 1511. https://doi.org/10.1021/jz500113x

[77] J. Wei et al., J. Phys. Chem. Lett. 5 (2014), 3937. https://doi.org/10.1021/jz502111u

[78] D. Forgacs, M. Sessolo, H. J. Bolink, J. Mater. Chem. A 3 (2015), 14121. https://doi.org/10.1039/C5TA03169A

[79] G. A. Sepalage et al., Adv. Funct. Mater. 25 (2015), 5650. https://doi.org/10.1002/adfm.201502541

[80] A. H. Ip et al., Appl. Phys. Lett. 106 (2015), 143902. https://doi.org/10.1063/1.4917238

[81] Z. Wei et al., J. Mater. Chem. A 3 (2015), 24226. http://dx.doi.org/10.1039/C5TA07714A

[82] J. H. Heo et al., Energy Environ. Sci. 8 (2015), 1602. http://dx.doi.org/10.1039/C5EE00120J

[83] H. Yoon, S. M. Kang, J.-K. Lee, M. Choi, Energy Environ. Sci. 9 (2016), 2262. http://dx.doi.org/10.1039/C6EE01037G

[84] Y. Rong et al., Energy Environ. Sci. 10 (2017), 2383. https://doi.org/10.1039/C7EE02048A

[85] D.-Y. Son et al., J. Am. Chem. Soc. 140 (2018), 1358. https://doi.org/10.1021/jacs.7b10430

[86] D. Yao et al., J. Phys. Chem. Lett. 9 (2018), 2113. https://doi.org/10.1021/acs.jpclett.8b00830

[87] X. Liu et al., Energy Environm. Sci. (2019). http://dx.doi.org/10.1039/C9EE00872A

[88] D.-H. Kang, N.-G. Park, Adv. Mater. 31 (2019), 1805214. https://doi.org/10.1002/adma.201805214

[89] O. Almora, L. Vaillant-Roca, G. Garcia-Belmonte, Rev. Cubana Fis. 34 (2017), 58.

http://www.revistacubanadefisica.org/index.php/rcf/article/view/RCF_34-1_58

[90] P. Lopez-Varo et al., Adv. Energy Mater. 8 (2018), 1702772.

https://doi.org/10.1002/aenm.201702772

[91] J. Carrillo et al., Adv. Energy Mater. 6 (2016), 1502246 https://doi.org/10.1002/aenm.201502246

[92] S. Ravishankar et al., J. Phys. Chem. Lett. 8 (2017), 915. https://doi.org/10.1021/acs.jpclett.7b00045

[93] C. I. Torres, A. K. Marcus, P. Parameswaran, B. E. Rittmann, Environ. Sci. Technol. 42 (2008), 6593. https://doi.org/10.1021/es800970w

[94] O. Almora, C. Aranda, E. Mas-Marzá, G. Garcia-Belmonte, Appl. Phys. Lett. 109 (2016), 173903. https://doi.org/10.1063/1.4966127

[95] O. Almora, C. Aranda, G. Garcia-Belmonte, J. Phys. Chem. C 122 (2018), 13450. https://doi.org/10.1021/acs.jpcc.7b11703

[96] O. Almora, M. García-Batlle, G. Garcia-Belmonte, J. Phys. Chem. Lett. 10 (2019), 3661. https://doi.org/10.1021/acs.jpclett.9b00601

[97] Y. Shao et al., Nat. Commun. 5 (2014), 5784. https://doi.org/10.1038/ncomms6784

[98] J.-W. Lee et al., Adv. Energy Mater. 5 (2015), 1501310. https://doi.org/10.1002/aenm.201501310

[99] B. Chen, H. Hu, T. Salim, Y. M. Lam, J. Mater. Chem. C 7 (2019), 5646. https://doi.org/10.1039/C9TC00816K

[100] B. Chen et al., Adv. Mater. 31 (2019), 1902413. https://doi.org/10.1002/adma.201902413

[101] M. Li et al., Adv. Mater. 31 (2019), 1901519. https://doi.org/10.1002/adma.201901519

[102] X. Zhou et al., Nano Energy 63 (2019), 103866. https://doi.org/10.1016/j.nanoen.2019.103866

[103] A. Kalam et al., Mater. Res. Express 6 (2019), 105510. https://doi.org/10.1088/2053-1591/ab334f

[104] H.-S. Duan et al., Phys. Chem. Chem. Phys. 17 (2015), 112. https://doi.org/10.1039/C4CP04479G

[105] Q. Dong et al., Chem. Mater. 31 (2019), 6833. https://doi.org/10.1021/acs.chemmater.9b01292

[106] H. Yu et al., Nano Energy 67 (2020), 104285. https://doi.org/10.1016/j.nanoen.2019.104285

[107] J. Xue et al., Appl. Optics 59 (2020), 552. https://doi.org/10.1364/AO.59.000552

[108] J. V. Li, G. Ferrari, Eds., Capacitance Spectroscopy of Semiconductors, Vol. 1, Taylor \& Francis Group, New York 2018. 
[109] T. P. Weiss et al., IEEE J. Photovoltaics 4 (2014), 1665.

https://ieeexplore.ieee.org/document/6915718

[110] S. Wang et al., J. Phys. Chem. C 122 (2018), 9795. https://doi.org/10.1021/acs.jpcc.8b01921

[111] J. Kneisel, K. Siemer, I. Luck, D. Bräunig, J. Appl. Phys. 88 (2000), 5474.

https://doi.org/10.1063/1.1312838

[112] T. P. Weiss et al., "Role of high Series Resistance in Admittance Spectroscopy of Kesterite Solar

Cells", presented at 2013 IEEE 39th Photovoltaic Specialists Conference (PVSC), Tampa, FL, USA, 2013. https://ieeexplore.iee.org/document/6745108

[113] A. Yelon, B. Movaghar, H. M. Branz, Phys. Rev. B 46 (1992), 12244.

https://link.aps.org/doi/10.1103/PhysRevB.46.12244

[114] R. Metselaar, G. Oversluizen, J. Solid State Chem. 55 (1984), 320. https://doi.org/10.1016/00224596(84)90284-6

[115] J. Heath, P. Zabierowski, in Advanced Characterization Techniques for Thin Film Solar Cells, Vol. 1 (Eds: D. Abou-Ras, T. Kirchartz, U. Rau), Wiley-VCH Verlag GmbH \& Co. KGaA, Boschstr. 12, 69469 Weinheim, Germany 2011, 81. https://doi.org/10.1002/9783527636280.ch4

[116] G. Garcia-Belmonte, J. Bisquert, ACS Energy Lett. 1 (2016), 683.

http://dx.doi.org/10.1021/acsenergylett.6b00293

[117] R. A. Awni et al., Joule 4 (2020), 644. https://doi.org/10.1016/j.joule.2020.01.012

[118] F. Werner et al., ACS Appl. Mater. Interfaces 10 (2018), 28553.

https://doi.org/10.1021/acsami.8b08076

[119] A. Guerrero et al., J. Phys. Chem. C 120 (2016), 8023. https://doi.org/10.1021/acs.jpcc.6b01728

[120] M. A. Mahmud et al., Sol. Energy Mater. Sol. Cells 159 (2017), 251.

https://doi.org/10.1016/j.solmat.2016.09.014

[121] A. Guerrero et al., App. Phys. Lett. 105 (2014), 133902. https://doi.org/10.1063/1.4896779

[122] S. Shao et al., Adv. Energy Mater. 8 (2018), 1702019. https://doi.org/10.1002/aenm.201702019

[123] S. J. Lee et al., ACS Energy Lett. 3 (2018), 46. https://doi.org/10.1021/acsenergylett.7b00976

[124] W. Li et al., J. Mater. Chem. A 4 (2016), 17104. https://doi.org/10.1039/C6TA08332C

[125] X. Du et al., Adv. Mater. (2020), 1908305. https://doi.org/10.1002/adma.201908305

[126] C. van Opdorp, Solid-State Electron. 11 (1968), 397. https://doi.org/10.1016/0038-1101(68)90020-8

[127] H. Kleemann, B. Lüssem, K. Leo, J. Appl. Phys. 111 (2012), 123722.

https://doi.org/10.1063/1.4730771

[128] Y. Yuan, J. Huang, Acc. Chem. Res. 49 (2016), 286. https://doi.org/10.1021/acs.accounts.5b00420

[129] A. Senocrate et al., J. Phys. Chem. C 122 (2018), 21803. https://doi.org/10.1021/acs.jpcc.8b06814

[130] A. Senocrate et al., Angew. Chem., Int. Ed. 56 (2017), 7755.

https://doi.org/10.1002/anie.201701724

[131] G. Gregori et al., in Organic-Inorganic Halide Perovskites Photovoltaics. From fundamentals to

Device Architectures, (Eds: Nam-GyuPark, M. Grätzel, T. Miyasaka), Springer, Switzerland 2016, 107. https://doi.org/10.1007/978-3-319-35114-8_5

[132] M. Fischer, K. Tvingstedt, A. Baumann, V. Dyakonov, ACS Appl. Energy Mater. 1 (2018), 5129. https://doi.org/10.1021/acsaem.8b01119

[133] W. Wei et al., J. Mater. Chem. A 5 (2017), 7749. https://doi.org/10.1039/C7TA01768E

[134] Y. Huang et al., EPJ Photovolt. 8 (2017), 85501. https://doi.org/10.1051/epjpv/2017001

[135] V. V. Brus et al., Adv. Electron. Mater. 3 (2017), 1600438. https://doi.org/10.1002/aelm.201600438

[136] A. Heinrichsdobler et al., in Organic Light Emitting Materials and Devices XXI, Vol. 10362 (Eds:

F. So, C. Adachi, J.-J. Kim), SPIE, 2017, 103622B.

http://spie.org/Publications/Proceedings/Paper/10.1117/12.2272976

[137] D. B. Khadka et al., J. Mater. Chem. C 5 (2017), 8819. 10.1039/C7TC02822A

[138] G. Yang et al., J. Mater. Chem. A 5 (2017), 1658. https://doi.org/10.1039/C6TA08783C

[139] A. S. Chouhan, N. P. Jasti, S. Avasthi, Mater. Lett. 221 (2018), 150.

https://doi.org/10.1016/j.matlet.2018.03.095

[140] A. Zohar et al., ACS Energy Lett. 4 (2019), 1. https://doi.org/10.1021/acsenergylett.8b01920 


\section{Chapter 5. Light characterizations and modeling}

From the dark characterizations in the previous chapter, the experimental evidence suggested that the current-voltage $(J-V)$ curve hysteresis in perovskite solar cells (PSCs) may be due to the slow kinetics of drifted ionic species accumulating towards the interfaces. These ionic phenomena produce a thermally activated significant increase of the low frequency capacitance spectra measured by impedance spectroscopy (IS). The ion migration competes with electronic currents and, more importantly, modifies the charge density profile, therefore conditioning the electronic current. Additionally, Faradaic currents cannot be neglected. However, the effects of $J-V$ hysteresis and the low-frequency capacitance in dark conditions are not so anomalously large as under illumination.

In this chapter, the hysteresis under standard illumination intensity is analyzed by changing the pre-polarization conditions. The experimental results are supported by numerical simulations, suggesting correlations between ionic dipolar switching and the recombination mechanisms. ${ }^{[1]}$ Subsequently, the IS as a function of illumination intensity is considered under different conditions and for several PSCs structures. ${ }^{[2]}$ Finally, a new approach named light intensity modulated impedance spectroscopy (LIMIS) is introduced and preliminary characterizations in PSCs are discussed. ${ }^{[3-4]}$

\subsection{Light hysteresis with dipolar switching experiment}

The shape of the $J-V$ curves for PSCs is very susceptible to several measurement parameters, for instance the direction, scan rate and light intensity during the voltage sweep, and the polarization history before the voltage sweep. ${ }^{[5-10]}$ One can refer to this issue as the multidependent hysteresis problem. Thus, typical characterizations requires the sampling of numerous variables which constitute a significantly time-consuming task. ${ }^{[6,9,11]}$ This is why most of the reported models are able to describe one particular $J-V$ hysteresis shape, or a family of patterns (see Figure 2.8), which in some cases are even of low reproducibility. $\left.{ }^{[8-9,}, 12-15\right]$

The herein called ionic dipoles switching measurement/modeling protocol avoids the multidependent hysteresis problem by taking two main considerations. First, the faster possible scan rate is employed for measuring $J-V$ curves, as pointed out in Section 4.2.2. ${ }^{[16]}$ This prevents capacitive displacement currents $J_{\text {cap }}$ and Faradaic non-capacitive contributions $J_{\text {noncap }}$, to affect the $J-V$ curve. Additionally, whatever the space-charge modifications due to ionic phenomena would be, they should not vary during the fast scan. Thus, an apparent quasi-steady state operational junction current $J_{j}$ can be defined. Second, a prior polarization time is set previously for each bias sweep. This pre-bias period should be higher than the relaxations of photocurrent and photovoltage after the applied voltage. Consequently, the analysis of the measured curves allows to differentiate the limit effects for processes happening in time scales longer or shorter than the voltage sweep time.

In this section, the analysis of $J-V$ curves from several perovskite solar cells measured at fast scan rates after pre-bias are presented. The studied devices present normal structure (see Figure 2.3a) with $\mathrm{CH}_{3} \mathrm{NH}_{3} \mathrm{PbI}_{3}$ (MAPI), $\mathrm{Cs}_{0.1} \mathrm{FA}_{0.74} \mathrm{MA}_{0.13} \mathrm{PbI}_{2.48} \mathrm{Br}_{0.39}$ (CFMPIB) and 
$\mathrm{FA}_{0.83} \mathrm{MA}_{0.17} \mathrm{~Pb}_{1.1} \mathrm{Br}_{0.22} \mathrm{I}_{2.98}$ (FMPBI) as absorber materials. Additionally, some of the studied devices included 2D capping layers towards the selective contacts (see Table 3.1), which were examined and associated with distinct types of ionic dipolar profiles. The MAPI-based devices show normal hysteresis (NHyst) while the mixed perovskite-based cells exhibit significant inverted hysteresis (IHyst) in the $J-V$ curves (see Section 2.4). Interestingly, the latter is highlighted for the FMPBI-based device, which are typically considered hysteresis-free devices due to the inclusion of organic selective contacts.

The main hysteresis feature under analysis is the large decrease of the short-circuit current $J_{s c}$ after negative pre-bias. The use of drift-diffusion simulations indicates that negative pre-biasing difficult the charge carrier collection towards the selective contacts. This boosts the charge carrier recombination rates in both the perovskite bulk and towards the electrodes. These behaviors can be understood in terms of bias-induced inversion of ionic dipole regions, for the case of NHyst. Contrarily, the IHyst of mixed-perovskite structures manifests larger $J_{s c}$ after negative pre-biasing because the dipolar switching is hindered.

In the following, the ranges of direct-current (DC) mode bias will be described as: forward/positive (F) bias for those applied voltages in the direction from short-circuit (SC) to open-circuit (OC) regimes. Similarly, reverse/negative (R) bias will be taken for those values of DC applied voltages below SC (see Chapter 2). Accordingly, the label RF is referred to the voltage sweep from SC to OC, and the opposite is FR. ${ }^{[1,9,13,17]}$ In the presence of hysteresis, one sweeps the applied voltage for the directions $\mathrm{RF}$ and $\mathrm{FR}$ and measures the corresponding current densities $J_{R F}(V)$ and $J_{F R}(V)$, which are different as

$$
\Delta J_{\text {hyst }}=J_{F R}-J_{R F}
$$

which is voltage dependent and dynamically evolving as $J_{j}$, whose general form is that of Equation 2.26.

The experimental $J-V$ curves measured at $5.0 \mathrm{~V} \cdot \mathrm{s}^{-1}$ after 1 minute stabilization at $-0.1 \mathrm{~V}$ and $1.2 \mathrm{~V}$ (see scheme of Figure 5.1a) are presented in Figure 5.1b,c. In this experiment, only processes faster than $200 \mathrm{~ms}$ may affect the shape of the $J-V$ curve. This means that the slower processes would "freeze".

An abrupt drop of photocurrent $\Delta J_{s c}$ after reverse pre-polarization (at - $0.1 \mathrm{~V}$ ) in the RF sweep is remarked for MAPI-cells with 2D capping in Figure 5.1b. This decrease is larger in 2DspiroMapi samples (see Table 3.1). Secondarily, the FF is reduced in the RF scan when comparing with to the FR sweep, independently on whether the 2D capping layers are included or not.

The NHyst occurs for every MAPI-based device in the voltage range from $\mathrm{SC}$ to OC, in Figure $5.1 \mathrm{~b}$, given $\Delta J_{\text {hyst }}>0$, as well as $\Delta J_{s c}$, from Equation (5.1). Absence of hysteresis would be $\Delta J_{\text {hyst }} \cong 0$. Thus, the inverted hysteresis (IHyst) implies $\Delta J_{\text {hyst }}<0$, as in the case of Figure 5.1c. ${ }^{[1,9,18]}$

The mixed perovskite samples show IHyst in their experimental $J-V$ curves, in Figure 5.1c. Negative values of $\Delta J_{s c}$ are significantly larger for the 2DspiroMix sample (see Table 3.1). Moreover, after the $1.2 \mathrm{~V}$ pre-bias the open-circuit voltage $\left(V_{o c}\right)$ is significantly reduced. Interestingly, this $V_{o c}$ decrease is quite evident in the PmixP sample (see Table 3.1). The latter 
structure, which include organic selective contacts, typically reports hysteresis-free $J-V$ curves when measured with slower sweep scan rates and without pre-bias. ${ }^{[16,19]}$
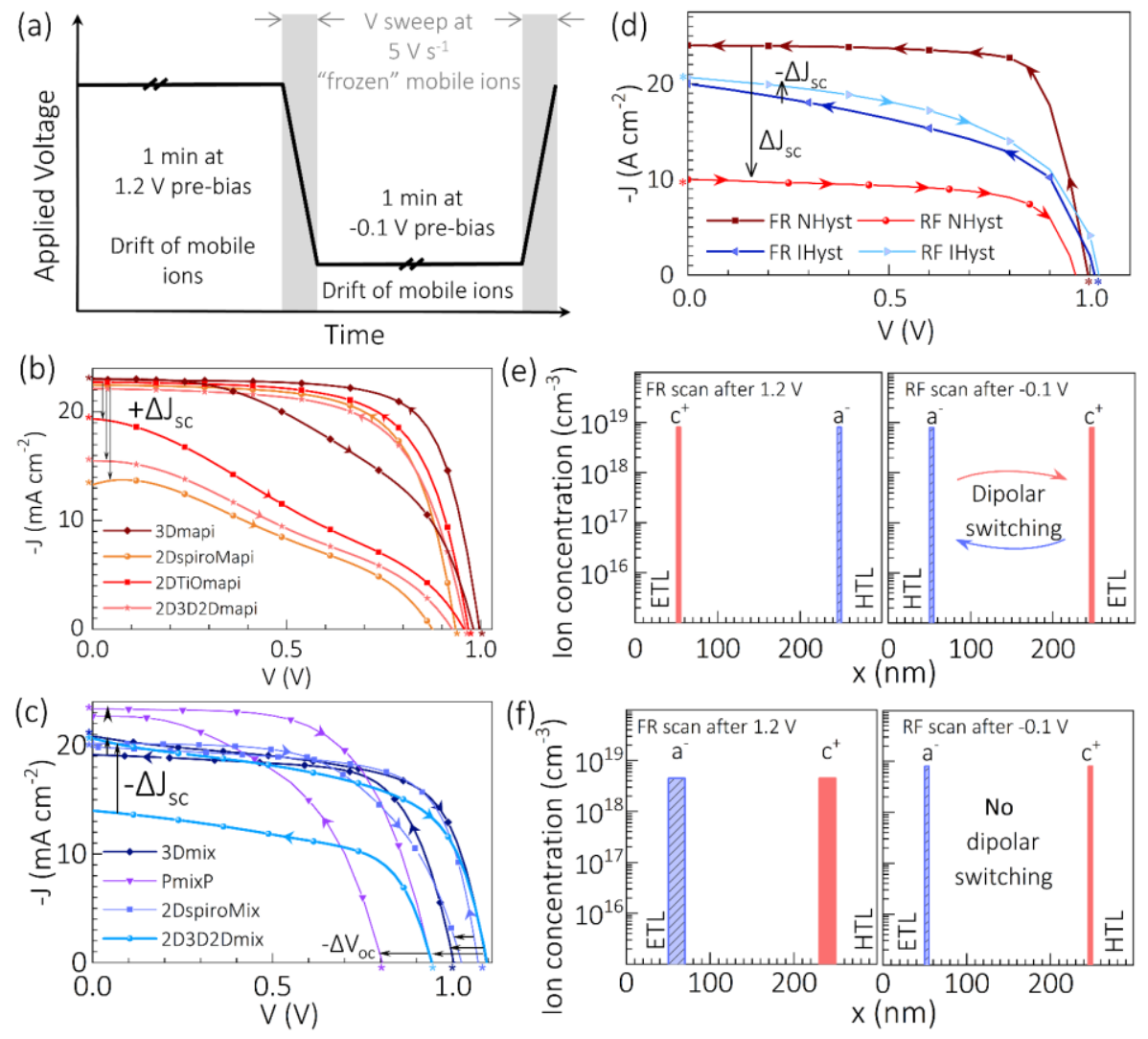

Figure 5.1. Light $J-V$ curves hysteresis and modeling: (a) experimental polarization concept, measured $J-V$ curves from (b) MAPI- and (c) mixed-perovskite-based devices, and (d) corresponding simulations for normal and inverted hysteresis with respective (e, f) ionic dipolar profiles. Adapted from ref. ${ }^{[1]}$, Copyright 2019, Elsevier B.V.

The recombination modes and the dielectric constants of the perovskites are the main differences between MAPI- and mixed perovskite-based cells, besides showing NHyst and IHyst, respectively. About recombination, the ideality factor $m \approx 2$ from MAPI-based solar cells suggest major importance of Shockley-Read-Hall (SRH) processes while in mixed CFMPIBbased devices larger importance of band-to band recombination is believed because $m \approx 1.5$, considering $V_{o c}$ values near the radiative limit, ${ }^{[1]]}$ as discussed in the next section. ${ }^{[2]}$ Regarding the dielectric permittivity, values of $\varepsilon>23$ are well known for MAPI (see Section 4.1.1), ${ }^{[20-23]}$ 
and $\varepsilon \approx 15$ for CFMPIB have been obtained experimentally and from first principles

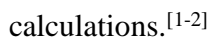

Numerical simulations were subsequently used for understanding the $\Delta J_{s c}$ behavior. In the model, MAPI-based cells with regular structure (see Section 2.2.1) were considered with the modification of including the 2D capping layers between the light harvesting perovskite and the selective contacts. ${ }^{[1,9,24-25]}$ Equations (2.10-19) were stationary solved with an immobile distribution of ions, simulating the quasi-steady-state during the fast scans after pre-bias. The resulting general trends are displayed in Figure 5.1d. Several dipolar distributions were assumed, placing the charges in sheets at the interfaces like in Figure 5.1e,f for each voltage sweep. ${ }^{[1,24]}$

The simulated $J-V$ curves of Figure 5.1d correspond to fast scans in the FR and RF directions, emulating the experimental hysteresis trends reported in Figure 5.1b,c. For the NHyst, the results correlate with ionic dipolar switching. This alludes to not just ions which pile up, but ions that specifically distribute depending on the device structure/materials and the polarization history. These ionic configurations are symmetrically switched, reversing the sign, during the prebias period in the experiment of Figure 5.1a. Note that this process also occurs when the $J-V$ curve measurements are performed at slower scan rates and without pre-bias, but in a more dynamical way.

Increase of charge carrier recombination is the main effect producing the large $\Delta J_{s c}$ during the RF sweep, after reverse pre-bias. ${ }^{[1]}$ In the situation of the right panel of Figure 5.1e, The dipoles trigger both the surface recombination at their location and the bulk recombination in between. This hinders the charge collection at the electrodes.

The presence of 2D capping layers seems to be a needed condition for the dipolar switching in MAPI-based devices with NHyst. One could correlate this behavior with low mobility values of the $2 \mathrm{D}$ layers ${ }^{[26-28]}$ that could slow down the drift of the mobile ions trapping them during the fast voltage sweep, after the corresponding pre-biasing. Purposely, the inhibition of ion migration in $2 \mathrm{D} \mathrm{PEA}{ }_{2} \mathrm{PbI}_{4}$ has been reported by electro-thermal studies. ${ }^{[29]}$ In addition, Muljarov et al. ${ }^{[27]}$ calculated by drift-diffusion simulations the charge carrier recombination rate constants around ten times higher for 2D perovskites than for the analogue 3D materials.

NHyst can numerically be easily reproduced, in wide ranges for the simulation parameters. ${ }^{[1]}$ This agrees with the fact that most of the experimental reports about hysteresis in PSCs are of NHyst. ${ }^{[9,13]}$ Contrary, the IHyst is not that often found in PSCs. ${ }^{[9,13]}$ Preliminarily, Rong et al. ${ }^{[18]}$ correlated IHyst with the nature of the ETL/perovskite interface, and Nemnes et al. ${ }^{[30]}$ pointed out that, unlike NHyst, the pre-conditioning state is essential in the IHyst.

For the IHyst to occur, several factors should combine: ${ }^{[1]}$ (i) a specific initial ion configuration created during the pre-biasing; ${ }^{[30]}$ (ii) specific values for the charge carrier surface recombination rates towards the selective contacts; ${ }^{[18]}$ (iii) a suitable range for the mobility of the charge carriers in the perovskite and (iv) an proper range of values for the dielectric constant of the perovskite. Note that, the event of IHyst needs these four factors, nevertheless each of the factors are not individually sufficient for IHyst to happen. ${ }^{[1]}$

Inhibition of dipolar switching seems to occur in mixed-perovskite solar cells, whose simulated IHyst in Figure 5.1d correspond to the charge distribution in Figure 5.1f. The pre-bias shrinks or widens the ionic dipoles when IHyst, but the sign is not switched in Figure 5.1f. The simulation of IHyst in mixed-perovskite cells was only possible when the values for the charge 
carrier mobility and dielectric constants were inevitably lesser than those utilized for reproducing the $J-V$ curves with NHyst for MAPI-based devices.

To sum up, the drift-diffusion numerical simulations can describe the main hysteresis experimental trends (NHyst, IHyst, $\Delta J_{s c}$ ) for current-voltage curves from perovskite solar cells measured with fast voltage scan rates after long pre-biasing, as in Figure 5.1b,c. The analysis correlates different types of light harvesting perovskites (MAPI or mixed) and respective ionic dipolar configurations, as an explanation for the hysteresis patterns. The approach above described is recommended as an experimental methodology for the characterization of the hysteresis phenomena in the current-voltage curve for perovskite solar cell.

\subsection{Light capacitance}

Light $J-V$ curve hysteresis in perovskite solar cells was showed in the previous section from several device structures. Hysteresis seems to be always present, even among top efficient cells and typically referred "hysteresis-free" cells. It is just a matter of select the right measurement condition and hysteresis will arise. More importantly, under illumination the hysteresis is able to modify tens of $\mathrm{mA} \cdot \mathrm{cm}^{-2}$ and hundreds of $\mathrm{mV}$ the apparent values for the $J_{s c}$ and the $V_{o c}$, respectively. Thus, one would expect larger capacitive features from the IS, analogously to the dark characterizations in the previous chapter.

In this section, the capacitance spectra of PSCs under illumination via IS are discussed for several cases and/or conditions. At open circuit, the close relation with recombination mechanism is addressed by using the diode ideality factor parametrization. ${ }^{[2]}$ Subsequently, a more general theoretical approach is proposed, accounting for the nature of light induced capacitances in PSCs.

\subsubsection{Impedance spectroscopy at open-circuit}

Similarly to Section 5.1, the present section discusses the characterization of devices comprising MAPI or mixed CFMPIB, as 3D perovskite absorbers, with 2D perovskite thin capping layers towards the $\mathrm{TiO}_{2}$ and/or the spiro-OMeTAD, as selective contacts. ${ }^{[2]}$ Distinctly, now the focus is on the IS spectra at open-circuit under different illumination intensities. Illustrative experimental spectra (dots) can be observed in Figure 5.2 in capacitance Bode plots and impedance Nyquist plots, with corresponding fittings (lines) to the equivalent circuit (EC) models.

For MAPI cells, with spectra like Figure 5.2a,b the most suitable EC model was that of Figure 5.2c, which is possibly the most common model for describing IS spectra in PSCs. ${ }^{[31-33]}$ In that EC, $L_{S}$ and $R_{S}$ are series connected inductor and resistor elements, respectively. Subsequently, two resistors in series $R_{L f}$ and $R_{H f}$ relates to the resistance contributions in the lower and higher frequency ranges, and similarly two capacitors $C_{L f}$ and $C_{H f}$ in matryoshka configuration, respectively. In general, at open circuit $C_{H f}$ correspond to the contributions from geometrical capacitances and/or electronic diffusion capacitance from the bulk. Similarly, $R_{H f}$ relates with recombination properties most likely in the bulk too. On the other hand, the origin of $C_{L f}$ and $R_{L f}$ 
has been more debated, but a correlation with interface phenomena and the slow ionic mechanisms is among the more often accepted. ${ }^{[9,23,34]}$

For frequencies above $100 \mathrm{~Hz}$, the capacitance spectra in the Bode plot describe a plateau around $C_{H f}$, as in Figure 5.2a. In the impedance Nyquist plot, as Figure 5.2b, a clear arc of diameter $R_{H f}$ is often observed above $100 \mathrm{~Hz}$ too. For frequencies lower than $100 \mathrm{~Hz}$, the capacitance significantly increases as the incident light intensity does, as in Figure 5.2a. One would expect a second plateau around $C_{L f}$ towards lower frequencies in the capacitance Bode plots and a second arc with approximate diameter $R_{L f}$ is apparent in the impedance Nyquist plot.
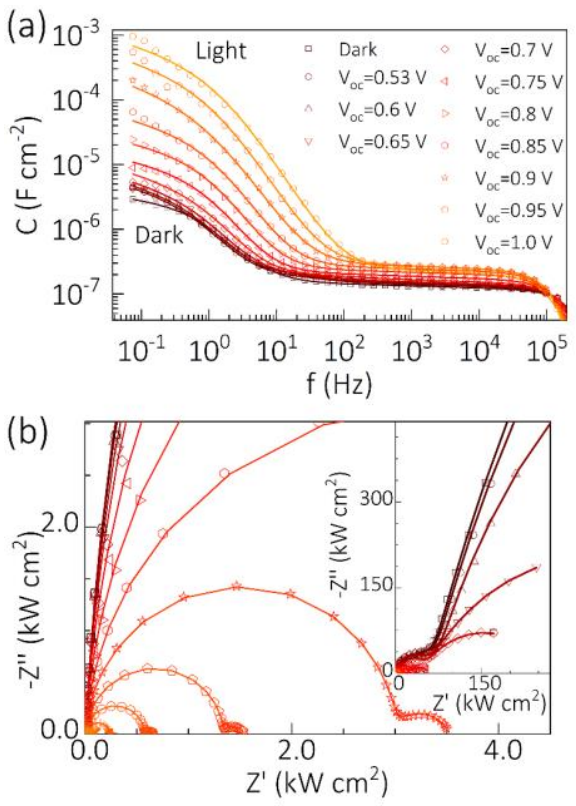

(c)

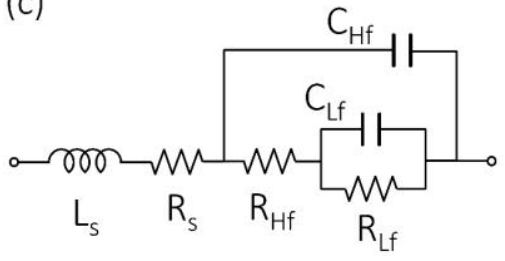

(f)
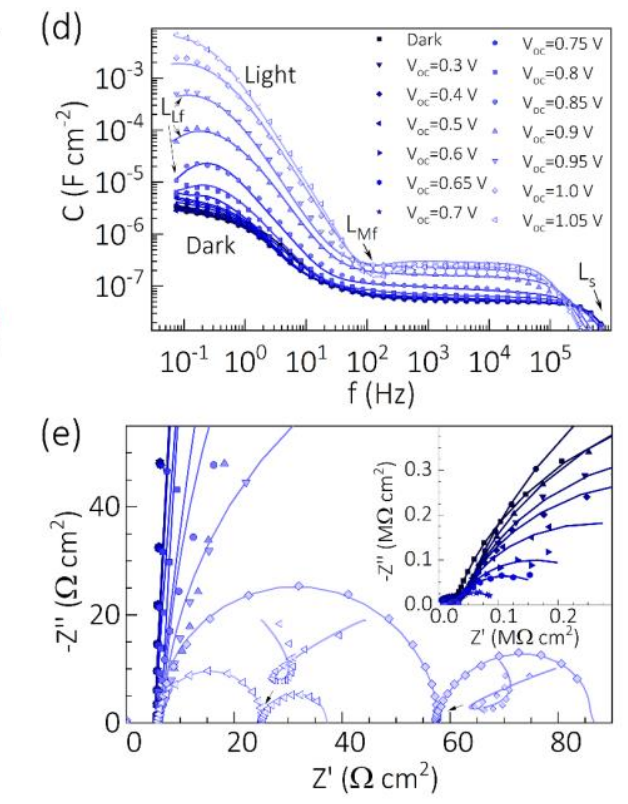

(d)

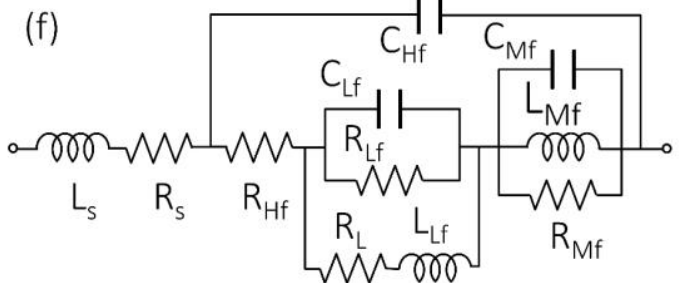

Figure 5.2. Impedance spectra of PSCs at open-circuit for different illumination intensities. Illustrative spectra (dots) for (a,b) MAPI devices and corresponding fittings (lines) to the equivalent circuit in (c). Similarly, illustrative spectra (dots) for (d, e) CFMPIB-based cells which are better fitted (lines) to the equivalent circuit in (f).

For CFMPIB-based cells, it results more appropriate to use the EC model of Figure 5.2f, which presents two main modification regarding that of Figure 5.2c. First, an inductive element 
$L_{L f}$ in series with the resistance $R_{L}$ are set in parallel with $C_{L f}$ and $R_{L f}$. These extra circuit elements modify the low frequency part of the spectra, as illustrated in Figure 5.2d. Second, another extra set of parallel resistor $R_{M f}$, capacitor $C_{M f}$ and inductor $L_{M f}$ are included as in Figure 5.2f. The latter circuit elements affect an intermediate range of frequencies between $10 \mathrm{~Hz}$ and 1 $\mathrm{kHz}$, reproducing the experimental loops in the impedance Nyquist plots and concave sections in the capacitance Bode plots (see Figure 5.2d).

The need for extra circuit elements in the AC model for simulating the spectra from CFMPIBbased samples seems to correlate with the varied composition of the mixed perovskite, in contrast with MAPI-based devices. Intuitively, one could expect that the common ionic contribution to the low frequency part of the capacitance is that of the iodine vacancies, which are abundant in MAPI as well as in the studied mixed perovskites. However, the differences in charge carrier mobility for each ionic specie may be compensated by electronic charge charrier kinetics, hence producing inductive-like behaviors. The latter has been connected to complex recombination/extraction phenomena taking place towards the selective contacts. $\left.{ }^{[13,}, 32,35-36\right]$

The behavior of the main resistive and capacitive contributions to the simulated spectra are displayed in Figure 5.3. The general exponentially increasing and decreasing behaviors for the capacitance and the resistance ${ }^{[31,37]}$ are highlighted with solid lines in Figure 5.3. For the capacitance at the lowest frequencies under illumination at open-circuit, a parametrization is proposed as

$$
C_{L f}=C_{s c i}+C_{L} \exp \left[\frac{q V_{o c}}{m_{c} k_{B} T}\right]
$$

where the exponential coefficient $m_{c}$, the equilibrium capacitance $C_{s c i}$ and $C_{L} \ll C_{s c i}$ (so $C_{L f} \cong$ $C_{s c i}$ in dark) serve as fitting parameters and $k_{B} T / q$ is the thermal voltage.

At low frequencies, $C_{L f}$ exhibits the exponential trend of (5.2) with $m_{c} \approx 2$ for MAPI-based cells (Figure 5.3a), resulting in large values up to $100 \mathrm{mF} \cdot \mathrm{cm}^{2}$ at $V_{o c} \approx 1.00 \mathrm{~V}$ under standard 1 sun light intensity. For the CFMPIB-based devices (Figure $5.3 \mathrm{~b}$ ), $m_{c} \approx 1.5$ and similar capacitances are reached under 1 sun, only that now $V_{o c} \approx 1.05 \mathrm{~V}$. For all the studied devices $C_{s c i}$ $\sim 10 \mu \mathrm{F} \cdot \mathrm{cm}^{-2}$, which was correlated with mobile ion space charge regions in Chapter 4. ${ }^{[34]}$

At high-frequencies, the $C_{H f}$ behavior in Figure 5.3a,b is nearly constant at forward bias. Furthermore, in all cases the lower values were those of the devices without extra $2 \mathrm{D}$ capping. Accordingly, one can suggest that geometric capacitance is the main contribution: $C_{H f} \approx C_{g}$. Nevertheless, minor contributions from electronic chemical capacitance cannot be fully discarded in these frequency ranges. ${ }^{[2,38-39]}$

The resistances are nearly constant at low illumination intensities/voltages when the shunt resistance $R_{s h}$ is predominant, e.g. Figure 5.3c. Above an onset voltage ( $\sim 0.4 \mathrm{~V}$ for MAPI cells and $\sim 0.7 V$ for CFMPIB cells) the resistance decrease exponentially in terms of Equation (2.25). This allows to estimate the ideality factor, which results $m \approx 2$ for the MAPI-based devices and $m \approx 1.5$ for the CFMPIB-based samples, nearly independently of the frequency range. Similarly, $J_{s c}-V_{o c}$ curves agree with these reports (see Equation 2.28). ${ }^{[2]}$

The natures of $R_{H f}$ and $R_{L f}$ under lower illumination intensities/voltages can be attributed to bulk and surface/interphase phenomena, respectively. Note that they are series connected, and 
more importantly $R_{L f} \gg R_{H f}$ making $R_{L f} \cong R_{S h}$, a well-known leakage current recombination mechanism. At higher illumination intensities, where the injection currents and bulk band-to-band radiative recombination earns importance, $R_{H f}>R_{L f}$ but not more than an order of magnitude. This could mean a lower importance of surface phenomena and/or a change in nature of $R_{L f}$.
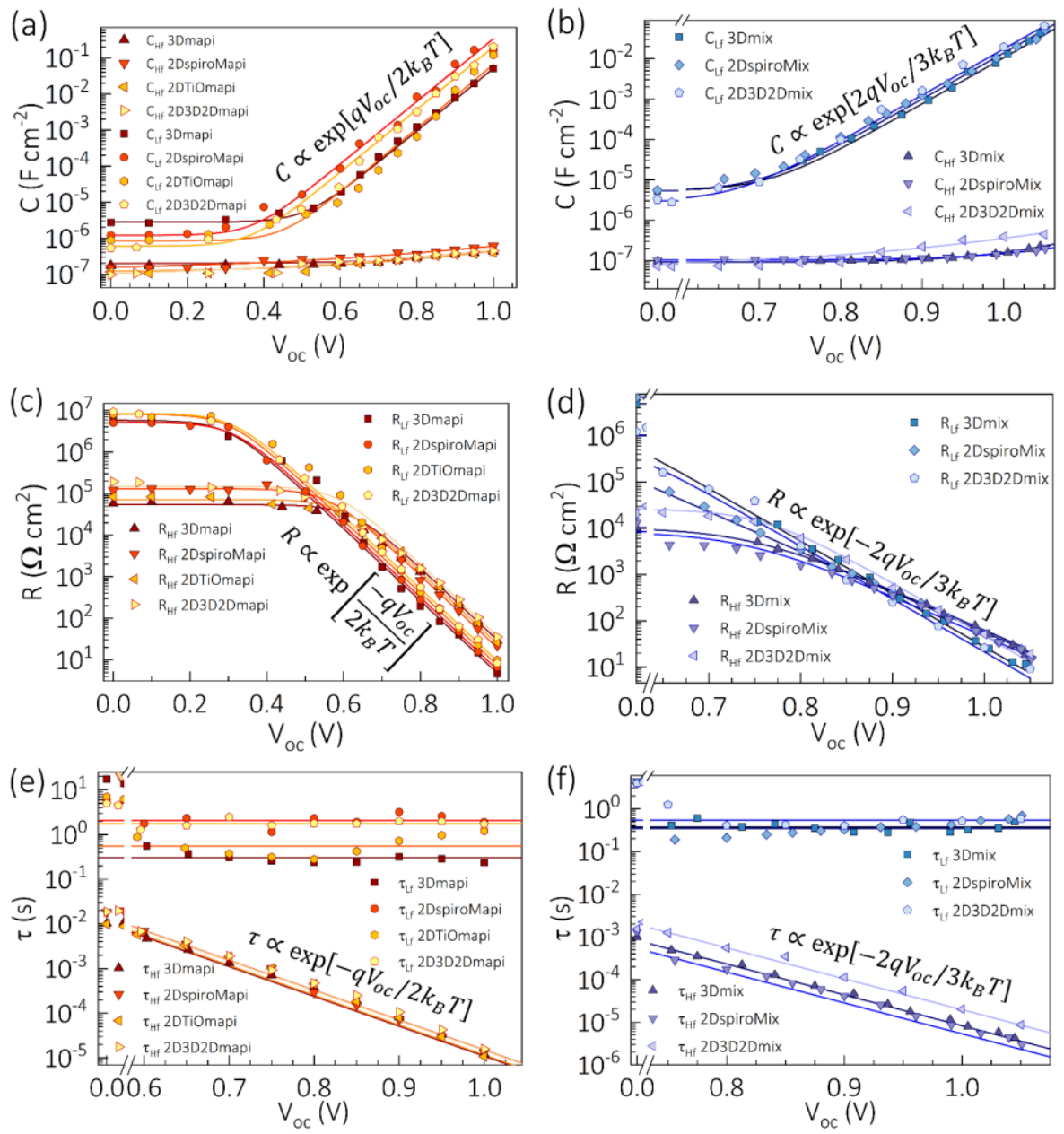

Figure 5.3. Parameters from equivalent circuit modeling of IS spectra of PSCs at open circuit: $(a, b)$ capacitance, $(c, d)$ resistance, and $(e, f)$ characteristic response times from MAPI- and mixed-perovskite-based devices, respectively. Dots are the fitting parameters and lines are exponential/linear fittings highlighting the general trends. See Table 3.1 for labeling. Adapted from ref. ${ }^{[2]}$, Copyright 2018, Elsevier Ltd. 
The corresponding characteristic times are shown in Figure 5.3e,f. For high frequencies and large illumination intensities/voltages the general trend is

$$
\tau=\tau_{0} \exp \left[-\frac{q V_{o c}}{m_{\tau} k_{B} T}\right]
$$

where $\tau_{0}$ is the equilibrium lifetime and $m_{\tau}$ is the lifetime ideality factor. Thus $m_{\tau} \approx m$ meaning $\tau_{H f} \propto R_{H f}$ from the nearly constant behavior of $C_{H f}$.

For the low-frequency RC couple $\tau_{L f}$, the values are up to the order of seconds and nearly constant with illumination intensities/voltages. These large times correlate $\tau_{L f}$ with slow mechanisms, most likely ionic, whose influence is enhanced under illumination. These mechanisms could also be assumed to be interface phenomena, since $C_{L f}$ couples with $R_{L f}$. Additionally, the constancy of $\tau_{L f}$ may suggest that $C_{L f} \propto R_{L f}{ }^{-1}$, from the fact that $m \approx m_{c}$ in the exponential trends of $R_{L f}$ and $C_{L f}$. In other words, a proportionality between low-frequency capacitance and surface recombination currents can be expected.

Whatever the nature of the large low-frequency capacitance at $\mathrm{OC}$ is, it may discard junction depletion capacitance and pure electronic chemical capacitance. Dismissing $C_{d l}$ is obvious due to the high forward bias regime (see sections 2.6.1, 3.3.2, 4.3.2). On the other hand, $C_{\mu}$ can be neglected because of the low intrinsic carrier concentration $n_{i} \approx 10^{5}-10^{6} \mathrm{~cm}^{-3}$ inferred for the parabolic band approximation (2.4) with effective conduction $N_{C}$ and valence $N_{V}$ band effective density of states of order $10^{18} \mathrm{~cm}^{-3}$, ${ }^{[40-41]}$ thermal energy $k_{B} T \approx 26 \mathrm{meV}$ and bandgap $E_{g} \approx$ $1.6 \mathrm{eV} \cdot{ }^{[9,42]}$ Following definitions in Section 2.6.3, one would need $n_{i}>10^{12} \mathrm{~cm}^{-3}$ in a $300 \mathrm{~nm}$ thick perovskite to attain capacitances above $\mathrm{mF} \cdot \mathrm{cm}^{-3} \cdot{ }^{[43]}$

Zarazua et al. ${ }^{[37]}$ proposed that $C_{L f}$ may be due to light-induced space-charge regions in ptype perovskites resulting in $m_{c}=2$. Subsequently, Jacobs et al., ${ }^{[44]}$ Moia et al., ${ }^{[45]}$ and Ebadi et al. ${ }^{[46]}$ independently used numerical drift-diffusion simulations and simulated the capacitance spectra by including large concentrations of mobile ions with slow kinetics in the perovskites.

To sum up, IS characterization of PSCs at open-circuit under different illumination intensities allows to identify bulk and interface features as well as recombination mechanisms. Bulk recombination resistance seems to be predominant at higher frequencies while interface/surface recombination resistance is suggested to respond at the lowest frequencies. The perovskite absorber layer is found to determine the main recombination pathway from the ideality factor parameter. Devices comprising $\mathrm{CH}_{3} \mathrm{NH}_{3} \mathrm{PbI}_{3}$ show $m \approx 2$, which can be assumed as predominant SRH recombination at surfaces and/or depleted regions. This was the behavior from all the MAPIbased samples. As listed in Table 3.1. On the other hand, a lower value, $m \approx 1.5$, is found for cells including mixed perovskites like $\mathrm{Cs}_{0.1} \mathrm{FA}_{0.74} \mathrm{MA}_{0.13} \mathrm{PbI}_{2.48} \mathrm{Br}_{0.39}$, which could suggest major influence of bimolecular recombination and/or diffusion mechanisms related with bulk properties. Similarly, all the mixed perovskites included in Table 3.1 presented the same trend.

Importantly, large capacitances of up to $100 \mathrm{mF} \cdot \mathrm{cm}^{2}$ are found at open-circuit under 1 sun illumination intensity. This capacitance could be related with photo enhanced large accumulation of mobile ions with slow kinetics. This, considering the light independent characteristic response 
times in the order of seconds, and/from the coupling with the low frequency surface recombination.

\subsubsection{Ionization, space charge regions and chemical capacitances}

The low-frequency capacitance of PSCs at open-circuit was showed in the previous section to increase exponentially with $V_{O c}$ reaching tens of $\mathrm{mF} \cdot \mathrm{cm}^{-2}$ under 1 sun illumination intensity. This is a well reported experimental feature in PSCs. ${ }^{[2,5,10,37,44-50]}$ However, large photo-induced capacitances are not an exclusive feature of the OC condition. ${ }^{[39]}$ The slow kinetics of mobile ions affect the complete operation of PSCs, causing hysteresis-like behaviors.

At short-circuit under illumination, there is also a significant increase in the photoinduced capacitance, as evident in the spectra of Figure 5.4a. In Figure 5.4b, one can see the linear trend of $C_{L f}$ towards the higher photon fluences. Contrary, below $1 \mathrm{~mW} \cdot \mathrm{cm}^{2} C_{L f}$ saturates towards the dark value. This is the typical behavior of traditional photovoltaics as Equation (2.51), only that $J_{\delta}<J_{s c}$ in PSCs. For instance, in Figure $5.4 \mathrm{~b}$ the device has $J_{s c}=22.5 \mathrm{~mA} \cdot \mathrm{cm}^{-2}$ and $J_{\delta} \approx 19.6$ $\mathrm{mA} \cdot \mathrm{cm}^{-2}$ for high frequencies while, more drastically, $J_{\delta} \approx 67.4 \mu \mathrm{A} \cdot \mathrm{cm}^{-2}$ for the low frequencies. The latter suggest that the nature of $C_{L f}$ may be ionic rather than electronic.
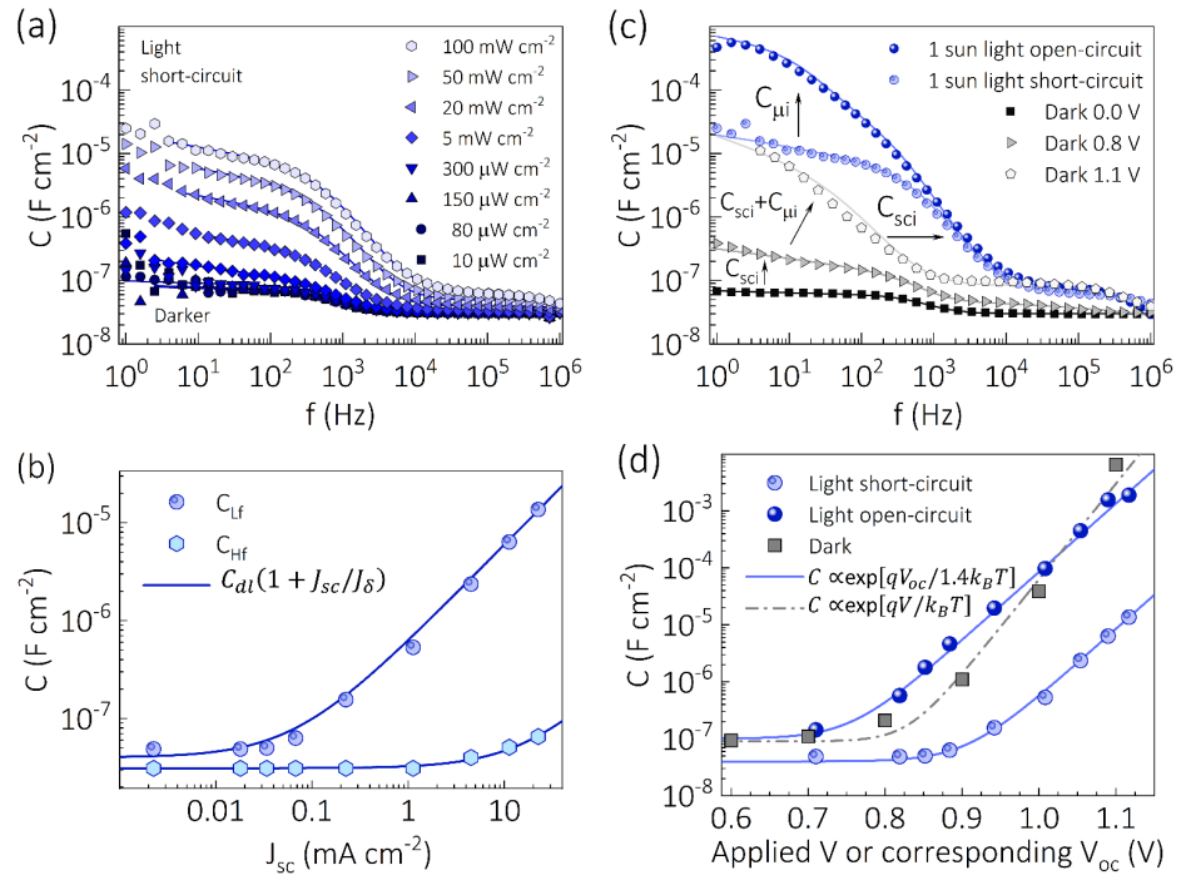

Figure 5.4. Mobile ions-related capacitances: (a) capacitance spectra of a PPmixP device (see Table 3.1) at SC under different illumination intensities and (b) corresponding capacitances at low and high frequencies as a function of short-circuit current; comparisons between (c) capacitance spectra at different conditions, as indicated, and (c) low-frequency capacitance 
from EC fittings as a function of applied DC bias or corresponding $V_{o c}$. Dots and lines in (a, c) are experimental data and fittings to EC of Figure 5.2c or Figure 4.7d.

Comparing the capacitance spectra at several conditions in Figure 5.4c illustrates different regimes for the low-frequency capacitance in PSCs. First, dark capacitance is slower than light capacitance, and it can be divided in two sub-regimes below and above flat-band condition. The dark situation for $V<V_{b i}$ follows an ionic depletion layer capacitance behavior, as already discussed in Section 4.3.2. Also in that section, it was discussed how the mobile ions can redistributed in the dark case when $V>V_{b i}$ and the space charge region is widened.

Light capacitance has a common faster component than dark capacitance, but at OC there is a larger and slower contribution than at SC. The results from the EC fittings are also summarized in Figure 5.4d, showing exponential trends with $m_{C} \approx 1.4$ for $C_{L f}$ at $\mathrm{OC}$ and SC (taking the corresponding $V_{o c}$ for each $P_{i n}$ ) and a faster increase for the dark $C_{L f} \propto \exp \left[q V / k_{B} T\right]$. Note that the latter is the general chemical capacitance trend when approaching high injection condition (see Section 2.6.3).

In common, it appears that increasing charge carrier by injection and/or photogeneration enhances the slow kinetic ionic phenomena. Purposely, light enhanced ionic transport was revealed by Zhao et al., ${ }^{[51]}$ who found a reduction in ionic transport activation energy from 0.82 $\mathrm{eV}$ in dark to $0.15 \mathrm{eV}$ under illumination for MAPI. Similarly, Xing et al. ${ }^{[52]}$ reported lower activation energies for ion conductivity of $0.15 \mathrm{eV}$ under illumination, with respect to $0.5 \mathrm{eV}$ in dark in large grain MAPI films. The ionic conductivity can be expressed as

$$
\sigma_{\text {ion }}=q_{\text {ion }} N_{\text {mob }} \mu_{\text {ion }}
$$

where $q_{i o n}$ is a multiple of the elementary charge which account for the ion charge, $N_{m o b}$ is the concentration of mobile ions and $\mu_{\text {ion }}$ their mobility. Then, assuming that $\mu_{\text {ion }}$ is not significantly changed, one could take most of the increase in the ionic conductivity due to a photo generated increase of concentration of mobile ions.

Photoionization is a process in which the energy of a photon is transferred to a bond electron, creating an ion. This process would require a photon energy larger than that of the given activation energy $E_{m o b}$. Another way to transfer an energy larger than $E_{m o b}$ to a bond electron is by impact with another electron with large enough kinetic energy. The impact electrons could be injected by field effect or photogenerated by the more energetic wavelengths. The later would also explain the increase of $N_{m o b}$ as illumination intensity is enhanced. A fourth option can also occur: a bond electron can be impacted by a mobile ion, which could produce an avalanche effect above certain value of $N_{m o b}$.

Note that $E_{m o b}$ is lower than the absolute ionization energy, since no particle is assumed to leave the lattice (there is not ionized gas). It would be enough for the bond electrons to reach the conduction band so the weakly bounded mobile ions can effectively move, drifted by the electric field.

The occurrence of photon-/electron-ionization in perovskites, and the exact involved mechanism, should be further verified by several methods. Nevertheless, from the available 
evidence and the above reasonings one could consider that the number of mobile ions is triggered as

$$
N_{m o b}=N_{m 0}+N_{m}\left(\frac{n \mathbb{P}_{n}+p \mathbb{P}_{p}}{n_{i}}+\frac{\Gamma_{i n}}{\Gamma_{b b}} \mathbb{P}_{\Gamma}\right)
$$

where $N_{m 0}$ is the equilibrium mobile ions concentration; $N_{m}$ is the equilibrium mobile ions ionization/activation concentration; $\Gamma_{i n}$ and $\Gamma_{B B}$ are the incoming and black-body radiation outcoming photon fluences, respectively; and $\mathbb{P}_{n}, \mathbb{P}_{p}$ and $\mathbb{P}_{\Gamma}$ are the probabilities of ionization/activation of the mobile ions by interaction with electrons, holes and photons, respectively.

Considering a proportionality (5.5) between charge carrier concentration and photon fluence with the number of mobile ions which contribute to low-frequency capacitance, and hence $J-V$ hysteresis in PSCs, makes easier to understand some processes. It would be just a matter of analyzing where the charge and photons are and how the mobile ions distribute, as in the energy diagrams and the charge density profiles of Figure 5.5.

(a)

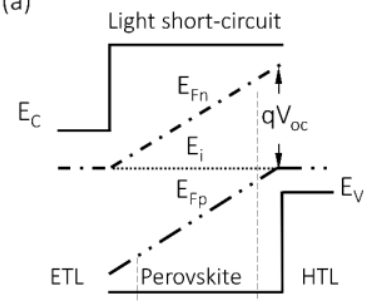

(b)

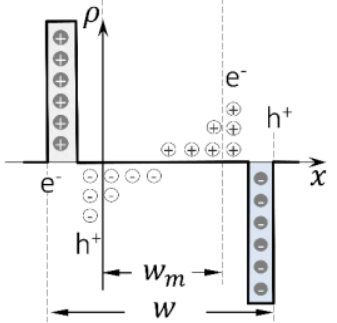

(c)

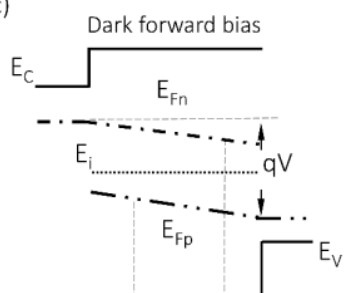

(d)

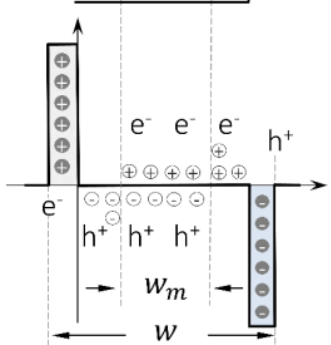

(e)

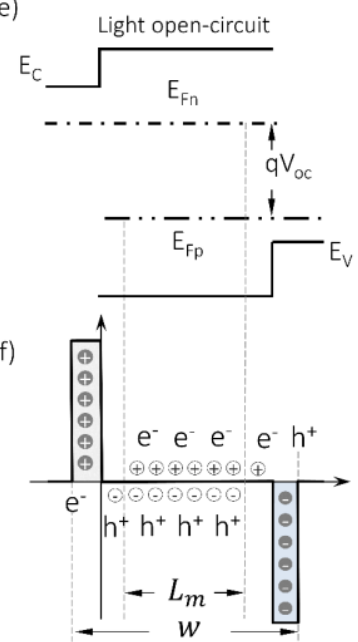

Figure 5.5. Schemed simplified energy diagrams (a, c, e) and charge density profiles (b, d, f) explaining the mobile-ions-related capacitances for $(a, b)$ light short-circuit, (c, d) dark forward bias and (e, f) light open-circuit conditions. Filled and open circles symbol fixed doping depleted ions and mobile ions, respectively, and $\mathrm{e}^{-}$and $\mathrm{h}^{+}$indicate electrons and holes, receptively. Not realistic band-bending, dipoles nor scaling were considered in the schemes.

At SC condition one could find the very simplified energy diagram of Figure 5.5a illustrating electrons and holes distributed towards the electrodes in order to provide the photocurrent. Here the charge carrier concentration and photon incidence could enhance the number of mobile ions which were already polarized by the built-in field. As a result, at SC and for the effective position 
$w_{m}$ where mobile ions respond to AC perturbation, one can propose a photo-enhanced mobile ions concentration

$$
N_{m o b}\left(w_{m}\right)=N_{m} \mathbb{P}_{m} \exp \left[\frac{E_{F n}\left(w_{m}\right)-E_{F p}\left(w_{m}\right)}{k_{B} T}\right]
$$

where $\mathbb{P}_{m}$ is the cumulative probability of ionization/activation of mobile ions producing an space-charge capacitance

$$
C_{s c i}=\sqrt{\frac{\varepsilon_{0} \varepsilon q N_{m} \mathbb{P}_{m}}{2 V_{b i}}} \exp \left[\frac{q V_{o c}}{m_{c} k_{B} T}\right]
$$

Here the parameter $m_{c}$ corrects the portion of the corresponding $V_{o c}$ proportional to the splitting of the quasi-Fermi levels at $w_{m}$, i.e. $E_{F n}\left(w_{m}\right)-E_{F p}\left(w_{m}\right)=2 q V_{o c} / m_{c}$ and $V_{o c}$ is the corresponding value under the same DC illumination intensity. Evidence of this photo-induced space charge formation can be found in the experiments by Tang et al. ${ }^{[53]}$ with thick absorber layer PSCs under different wavelength illumination intensities.

At dark forward biases above flat-band, the injection supplies the charge carriers that could reproduce an space charge capacitance as (5.7). However, the distribution of mobile ions may be wider and diffusion-type mechanisms cannot be discarded due to the Warburg like behavior of the constant phase element fitting $C_{L f}$ and the lower exponential coefficient $m_{c} \approx 1$.

At OC under illumination, the mechanism of (5.7) could still be occurring, most likely towards the interface, where most of the high energy photon absorption occurs. This would be similar to the situation suggested by Zarazua et al. ${ }^{[37]}$ However, additionally, the slowest and largest capacitance contributions could be due to a different mechanism, related with the broader and nearly constant concentration of mobile ions.

A chemical potential of ions whose concentration is analogue to (5.6), but constant along a $L_{m}$-thick region, can be defined as

$$
\mu_{i}=k_{B} T \ln \left[\frac{N_{m o b}}{N_{m}}\right]=k_{B} T \ln \left[\mathbb{P}_{m}\right]+E_{F n}-E_{F p} \cong q V_{o c}
$$

The approximation in Equation (5.8) is made assuming large ionization probability, i.e. $\mathbb{P}_{m}$ close to one, and perfect contact selectivity, i.e. $E_{F n}-E_{F p}=q V_{o c}$. Therefore, one can consider a chemical capacitance of mobile ions at open circuit as

$$
C_{\mu i} \cong q L_{m} \frac{\partial N_{m o b}}{\partial V_{o c}}
$$

where $L_{m}$ is the region where the concentration of both mobile anions and cations is equal and nearly constant. This region is not necessary the complete perovskite thickness because the probability $\mathbb{P}_{m}$ may be position-dependent even in regions where the electronic chemical potentials are flat. Thus, one can assume a dependency similar to (5.6) within the $L_{m}$ region and take $\mathbb{P}_{m}$ as an average value, resulting

$$
C_{\mu i}=\frac{q^{2} L_{m} N_{m} \mathbb{P}_{m}}{k_{B} T} \exp \left[\frac{q V_{o c}}{m_{c} k_{B} T}\right]
$$


where $m_{c}$ can account for $V_{o c}$ differences with the splitting of quasi-Fermi levels and/or be an effective correction due to $\mathbb{P}_{m}$.

In closing, the low-frequency capacitance of PSCs can be explained as a combination of space-charge capacitances and chemical capacitances due to ionization/activation of mobile ions. The latter seems to respond proportionally to the concentration of charge carriers. Specifically, due to just-generated charge carriers or direct photoionization the photon interaction with photovoltaic perovskites seems to explain the unprecedented large low frequency capacitances.

\subsection{Light intensity modulated impedance spectroscopy (LIMIS)}

Impedance spectroscopy analyses to photovoltaic devices are usually used to discern the predominant recombination mechanisms, ${ }^{[2]}$ the doping densities, ${ }^{[54-55]}$ deep defect levels ${ }^{[56]}$ and the density of states, ${ }^{[57]}$ as introduced in Section 3.3 and previously in this chapter. With the focus in the recombination phenomena, the measurement of potentiostatic IS at quasi-open-circuit regime for a series of DC illumination intensities (see Section 5.2.1) is one of the most stablished procedures. The experimental spectra can be simulated with EC models and so the different components for the recombination resistance $R_{r e c}$, chemical capacitance $C_{\mu}$ and characteristic charge carrier recombination lifetimes $\tau$ can be assessed as a function of $V_{o c}{ }^{[2,4,38]}$ Alternatively to the EC modeling, drift-diffusion numerical simulations can also be performed. One can similarly fit the experimental spectra of the time-dependent solutions for the transport equations at OC, with the same DC illumination and under small perturbation $\tilde{V}$ of the bias boundary condition $\bar{V}$ at the electrodes. ${ }^{[44-45,58-59]}$

In photo-sensitive samples one can also study the photocurrent or photovoltage responses to small alternating current (AC) mode light intensity perturbations over a DC illumination intensity. Then one can perform measurements of intensity modulated photocurrent and photovoltage spectroscopies, IMPS ${ }^{[60-71]}$ and IMVS, ${ }^{[66-67,72-74]}$ which describe the current and voltage responsivities $\Psi_{J}$ and $\Psi_{V}$, respectively. Similarly to IS, one can consider a light intensity modulated impedance spectroscopy (LIMIS) as the ratio LIMIS=IMVS/IMPS. The LIMIS relation was first presented by Song \& Macdonald ${ }^{[75]}$ who measured the spectra on $\mathrm{n}-\mathrm{Si}$ in $\mathrm{KOH}$ solution and validated the transfer function by Kramers-Kronig transformation. Subsequently, Halme $^{[76]}$ applied the concept to dye sensitized solar cells, and concluded a correspondence between the measurement methods for IS and LIMIS.

In the next sections, first, an analytical approximate solution for the one-sided p-n junction photovoltaic devices is presented. This formalism complements the EC-based approaches and sets an opening towards forthcoming more accurate numeric drift-diffusion simulations. Subsequently, the difference between the transfer functions resulting from IS and LIMIS are shown to inform on the properties of the junction, like the recombination velocity. ${ }^{[3]}$ Subsequently, the experimental spectra from IS and LIMIS for PSCs are discussed. Particularly, the corrections to the assessment of the charge carrier lifetimes are addressed and compared by different methods. 


\subsubsection{Theoretical introduction to LIMIS: the one-sided p-n junction case}

Following the impedance definition (3.3) and Equations (3.7-10) defining IMPS and IMVS, the current and the voltage are individually measured under small light perturbation around open circuit condition. Then, one can obtain the photo impedance transfer function of LIMIS=IMVS/IMPS as ${ }^{[3]}$

$$
Z_{\Psi}(\omega)=\frac{\Psi_{V}}{\Psi_{J}}=\frac{\left|\tilde{V}_{o c}\right|}{|\tilde{J}|} e^{i\left(\phi_{V}-\phi_{J}\right)}=\left|Z_{\Psi}\right| e^{i \phi_{\Psi}}
$$

where $Z_{\Psi}$ is a photo-impedance and $\phi_{\Psi}$ the corresponding phase shift.

Applying this concept to photovoltaic solar cells requires solving the drift-diffusion equations with the appropriate boundary conditions which reflect the device under study. Phenomenologically, during a light perturbation (in a third axis) the complete $J-V$ curve projection is down shifted as photocurrent increases, as in Figure 5.6a. In a 3D representation one can see that LIMIS can only be performed at the intersection between the open-circuit plane with current-voltage-incident light power $\left(J-V-P_{\text {in }}\right)$ surface, like black dots in Figure 5.6b.

Deriving $Z_{\Psi}$ requires first to calculate the current and photovoltage under AC perturbation. Using (2.15), ${ }^{[7]}$ one can obtain the $\mathrm{AC}$ current as ${ }^{[3]}$

$$
\tilde{J} \cong q \int_{0}^{\bar{d}}\left[\tilde{G}-\tilde{n}\left(\frac{1}{\tau}+2 \beta \bar{n}_{0}+i \omega\right)\right] d x
$$

Here $q$ is the elementary charge, $\bar{d}$ is the effective current density integration distance, $\tilde{G}$ the AC charge carriers generation rate, $\tau$ the non-radiative SRH recombination lifetime, $\beta$ the radiative recombination coefficient, $i=\sqrt{-1}$ is the imaginary unit, $\omega$ the angular frequency, $x$ the position in the direction of the current, $\bar{n}_{0}$ the steady-state or DC mode charge carrier density (under DC bias and/or DC illumination) and $\tilde{n}$ is the complex AC amplitude for the charge carrier density response to the AC small voltage perturbation $\tilde{V}$ in IS, or the AC small light intensity power perturbation $\tilde{P}_{i n}$ in LIMIS. Note that, upon perturbation, $\tilde{n}=|\tilde{n}| \exp \left[i \phi_{n}\right]$ being $\phi_{n}$ the phase shift with respect to $\tilde{V}$ in IS, or $\widetilde{P}_{i n}$ in LIMIS.

Also, setting $J=0$ in (2.20) one can use the McLaurin series to obtain the modulated photovoltage as ${ }^{[3,73]}$

$$
\tilde{V}_{o c} \cong 2 \frac{k_{B} T}{q} \frac{\tilde{n}}{\bar{n}_{0}}
$$

The method here aims solving the transport equations for obtaining $\bar{n}_{0}$ and $\tilde{n}$ in the DC and AC solutions, respectively. Then one can substitute $\bar{n}_{0}$ and $\tilde{n}$ in (5.11-5). Interestingly, the difference between IS and LIMIS lies in "where" the perturbation is applied to the system. For the simulation of potentiostatic IS measurements, $\tilde{V}$ relates to the electrostatic potential $\varphi$ boundary condition. Then the consequent modification in the electric field $\xi$ alters the charge carrier profiles through the Poisson's equation. Contrastingly, for LIMIS the perturbation $\tilde{P}_{i n}$ directly affects the continuity equation through the modification of the charge carrier generation rate $G$ within the effective absorber layer bulk length. The correlation between $\tilde{P}_{\text {in }}$ and the 
corresponding AC modulated generation rate $\tilde{G}$ can be assumed to be linear. Then, assuming that incident light spectrum does not change with the light intensity, $G$ can be expressed as

$$
G(t)=\bar{G}+\tilde{G} e^{i \omega t}=\frac{\Psi_{s c}}{q \bar{d}}\left(\bar{P}_{i n}+\tilde{P}_{i n} e^{i \omega t}\right)
$$

where $\bar{G}$ and $\tilde{G}$, and $\bar{P}_{\text {in }}$ and $\tilde{P}_{\text {in }}$ are the DC and AC components of the generation rate and the incident light power densities, respectively, $\bar{d}$ is the effective absorber layer thickness where the current is integrated, $t$ is the time and $\Psi_{s c}$ is the photocurrent responsivity at SC. Note that $\Psi_{S c}$ varies with the spectrum of $\bar{P}_{i n}$, and both the absorption coefficient and the geometry of the absorber(s). Additionally, (5.14) is most useful for thin film devices where a space independent constant $G$ is a good approximation, otherwise one should consider the Beer-Lambert law (2.18a). Furthermore, since $\tilde{G}=|\tilde{G}|$ and $\tilde{P}_{\text {in }}=\left|\tilde{P}_{\text {in }}\right|$ are the perturbation, similarly to $\tilde{V}=|\tilde{V}|$, the modulus notation is ignored in the following.

In order to separate the $\mathrm{DC}$ and $\mathrm{AC}$ parts of the analytical solution of transport equations the charge carrier concentrations can be written in the form

$$
n(t)=\bar{n}_{0}+\tilde{n} \exp [i \omega t]
$$

where $\bar{n}_{0}=\bar{n}+n_{0}, n_{0}$ is the dark equilibrium concentration and $\bar{n}$ the over-equilibrium DC component.

For the current boundary condition, one can consider an ohmic contact selectivity for both IS and LIMIS at OC, where $D \partial \tilde{n} / \partial x \cong S_{r} \tilde{n}$. The latter condition expresses that the diffusion current, with diffusion coefficient $D$, equals the recombination current with surface recombination velocity $S_{r}$ at the interface.
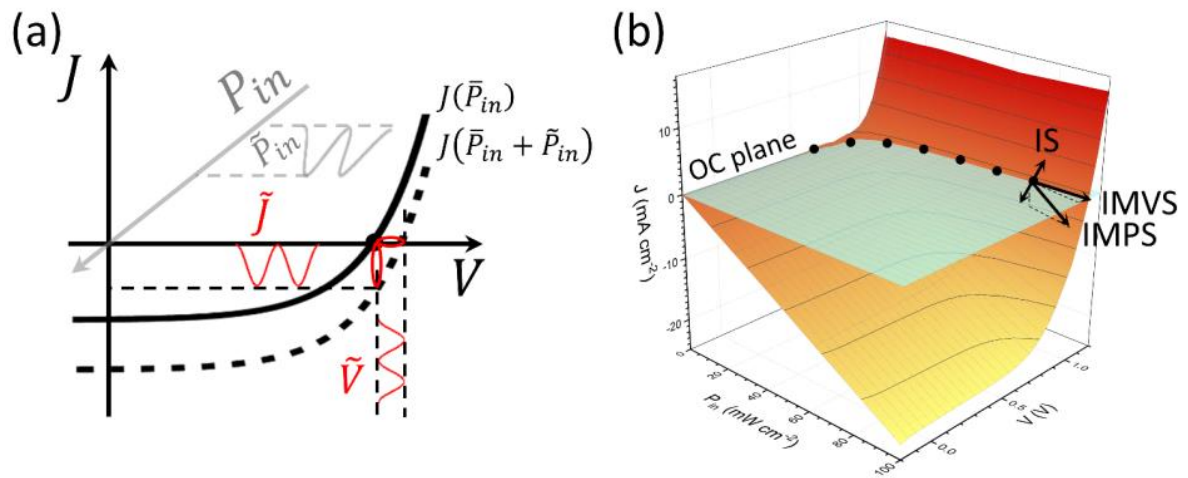

Figure 5.6. Introduction to LIMIS: (a) projection of the $J-V$ curve during small light perturbation, and (3) complete 3D plotting of the $J-V-P_{\text {in }}$ surface intercepting the open circuit plane. Dots in (b) indicate where the LIMIS can be measured.

The depletion approximation was considered as boundary condition for the electrostatic potential, in a form of voltage. This boundary condition carriers the information that separates IS from LIMIS, as they are measured in different ways. Consequently, one would expect different 
distributions for the charge carrier densities, and hence the energy diagrams. ${ }^{[3]}$ For IS, the perturbation $\tilde{V}$ modifies the DC width of the depletion region $\bar{w}$ to oscillate with an AC amplitude $\widetilde{w}$. This generates small gradients in the charge carrier density across the quasi-steady-state open circuit regime. For the IMVS, $\widetilde{G}$ also changes $\widetilde{w}$ around $\bar{w}$, but now there are no gradients in the charge carrier density in the quasi-neutral region, so there is no current. Finally, for IMPS, no change at the edge of the depletion region $\bar{w}$ is assumed and the effect of the perturbation create gradients of charge carrier concentration in the opposite sign, i.e. photo current opposes the injected recombination current.

Accordingly, the impedance from potentiostatic IS at OC results as

$$
Z(\omega) \cong \frac{2 k_{B} T}{q^{2} \bar{L}_{d} \bar{G}} \frac{1}{\tilde{\gamma} \sqrt{1+i \frac{\omega}{\omega_{0}}}}
$$

Here $\bar{L}_{d}=\sqrt{D / \omega_{0}}$ is the diffusion length from the low frequency limit, $\omega_{0}=$ $\left(\tau^{-2}+4 \bar{G} \beta\right)^{1 / 2}$ a characteristic recombination angular frequency and $\tilde{\gamma}$ is a surface recombination factor as calculated by Almora et al. ${ }^{[3]}$ Later, the respective photovoltage and photocurrent responsivities result as

$$
\Psi_{V}(\omega)=2 \frac{k_{B} T}{q \bar{G}} \frac{1}{\left(1+i \frac{\omega}{\omega_{0}}\right)}
$$

and

$$
\Psi_{J}(\omega)=\frac{q \bar{L}_{d}}{\sqrt{1+i \frac{\omega}{\omega_{0}}}} \tilde{\gamma}(1-\tilde{\delta})
$$

with the complex diffusion length $\tilde{L}_{d}=\bar{L}_{d}\left(1+i \omega / \omega_{0}\right)^{1 / 2}$ and

$$
\tilde{\delta}=S_{r} \frac{\left(e^{\frac{\bar{w}}{\tilde{L}_{d}}-e^{\frac{L-\bar{w}}{\tilde{L}_{d}}}}\right)}{\left(\frac{D}{\tilde{L}_{d}}+S_{r}+\left(\frac{D}{\tilde{L}_{d}}-S_{r}\right) e^{\frac{L}{\widetilde{L}_{d}}}\right)}
$$

Note that $\Psi_{V} \propto\left(1+i \omega / \omega_{0}\right)^{-1}$ and $\Psi_{J} \propto\left(1+i \omega / \omega_{0}\right)^{-1 / 2}$ behave with arc-shaped spectra in the Nyquist representation. In DC terms, (5.17) suggest $\Psi_{V} \propto \bar{G}^{-1}$ at the low frequency limit but $\Psi_{J}$ should be nearly $\bar{G}$-independent, as (5.18). Interestingly, one can use the low frequency limit of the IMVS spectra as a measurement method to evaluate the ideality factor $m$, given the relation ${ }^{[3]}$

$$
\Psi_{V} \cong m \frac{k_{B} T}{q \bar{G}}=m \frac{k_{B} T \bar{d}}{\Psi_{s c} \bar{P}_{i n}}
$$

Subsequently, LIMIS transfer function can be obtained as 


$$
Z_{\Psi}(\omega) \cong \frac{2 k_{B} T}{q^{2} \bar{L}_{d} \bar{G}} \frac{1}{\tilde{\gamma} \sqrt{1+i \frac{\omega}{\omega_{0}}}(1-\tilde{\delta})}
$$

Note that the photoimpedance as (5.21) for LIMIS differentiates from the potentiostatic impedance for IS as (5.16) in only a factor: $Z_{\mathrm{IS}}=Z_{\Psi} /(1-\tilde{\delta})$. Keeping this in mind, it is easy to formulate an expression to characterize the impedance difference between IS and LIMIS as

$$
\Delta Z_{\Psi}=\frac{Z_{\Psi}-Z}{Z}=\frac{\tilde{\delta}}{1-\tilde{\delta}}
$$

Importantly, by substituting (5.19) in (5.22) it is evident that $\Delta Z_{\Psi} \propto S_{r}$. This suggest that $\Delta Z_{\Psi}$ could be used as a figure of merit in order to characterize the surface recombination velocity at the electrodes.

In summary, the transport equations can be analytically solved for the simplest case of p-n junction solar cells under DC illumination at OC with small light and bias perturbations. The resulting time-dependent solutions were used to define the transfer functions for IS and LIMIS, whose difference in terms of impedance is proportional to recombination velocity at the interface.

Experimentally, for the measurement of LIMIS, first the cell is set under DC illumination intensity $\bar{P}_{\text {in }}$ till thermal equilibrium is made. Then, the steady-state is perturbed with a small sinusoidal AC light signal with amplitude $\tilde{P}_{\text {in }}$ at the same frequencies for both measurements: IMVS and IMPS. In the case of IMVS, the workstation sets absolute OC and the AC photovoltage response signal is sensed. For IMPS, the measurement is made in a quasi-open-circuit regime, where the absolute DC currents should be around nA or lower. This is achieved by applying the DC voltage $\bar{V}=\bar{V}_{o c}$ corresponding to that $\bar{V}_{o c}$ under $\bar{P}_{i n}$ at absolute OC. Then, the workstation measures the photocurrent signal due to $\tilde{P}_{i n}$ while keeping the corresponding $\bar{V}$. In the case of potentiostatic IS (see Section 3.3) the quasi-open-circuit regime is set like in IMPS, and the current signal due to $\widetilde{V}$ is measured while keeping the respective $\bar{V}$ for each $\bar{P}_{i n}$. In this way, several values of $\bar{P}_{i n}$ are explored and a different $\bar{V}_{o c}$ is obtained in each case.

Importantly, special attention should be paid to the linearity of the small perturbation when measuring IMPS and IMVS to obtain LIMIS. As a reference, for IS one usually takes $\tilde{V}<k_{B} T / q$, and for IMPS and IMPV it is advisable to keep $\tilde{P}_{i n}<\bar{P}_{\text {in }} / 10$. However, the latter can be problematic when $\bar{P}_{\text {in }}$ is close to the lowest value for $\tilde{P}_{\text {in }}$ which can be settled by the experimental setup. This is solved in the Zahner setup (see Section 3.3.5) by implementing the significance parameter (SP) as defined by Schiller and Kaus. ${ }^{[78]}$ This SP is reported per each measurement point (frequency) in the range from 0 to 1 , meaning "perfect linearity" when it equals unity. In practice, one can consider SP $>0.98$ as an optimal result and those $\mathrm{SP}<0.95$ should be rejected.

The IS, IMPS and IMVS measurements in the following section were performed with the Zahner Zennium Pro/PP211 workstation (see Section 3.3.5) utilizing the LSW-2 white LED light source. In all the cases the sample holder included $\mathrm{N}_{2}$ atmosphere (see Figure $3.6 \mathrm{c}$ ) in order to reduce moisture-induced degradation. 


\subsubsection{LIMIS and IS spectra in perovskite solar cells}

This section analyzes the IS and LIMIS spectra at open circuit under different illumination intensities for PSCs comprising mixed-perovskites as absorber layers. Differently to the devices studied in section 5.2.1, the here studied cells have $\mathrm{SnO}_{2}$ and PDCBT in the selective contact structures, being PPmixP the best performing and stable structure (see Table 3.1). Note that these devices are typically known as "hysteresis-free" PSCs, despite the can show significant hysteresis in as PmixP in Figure 5.1b.

The IMPS and IMVS spectra from PSCs at open-circuit evidence the presence of at least two main processes with well-defined different time scales. This is illustrated with representative voltage and current responsivities in the Nyquist plots of Figure 5.7, featuring two arcs for IMVS and IMPS. Interestingly, while IMVS resembles the typical impedance spectra, where the high frequency limit stays in the first quadrant of the Nyquist plot, the IMPS trend indicate that at high frequencies the spectra may spread to the second quadrant.

The low frequency limits are also represented in Figure 5.8a. The IMVS gives $\Psi_{V} \propto P_{\text {in }}{ }^{-1}$, in agreement with (5.20). Noticeably, for the IMPS the behaviour is somehow in the middle between constant $\Psi_{J}$ at lower light intensities and $\Psi_{J} \propto P_{i n}{ }^{-1 / 3}$ at higher illuminations. The latter suggest that the model in the previous section should be numerically refined in order to meet the experimental behavior.
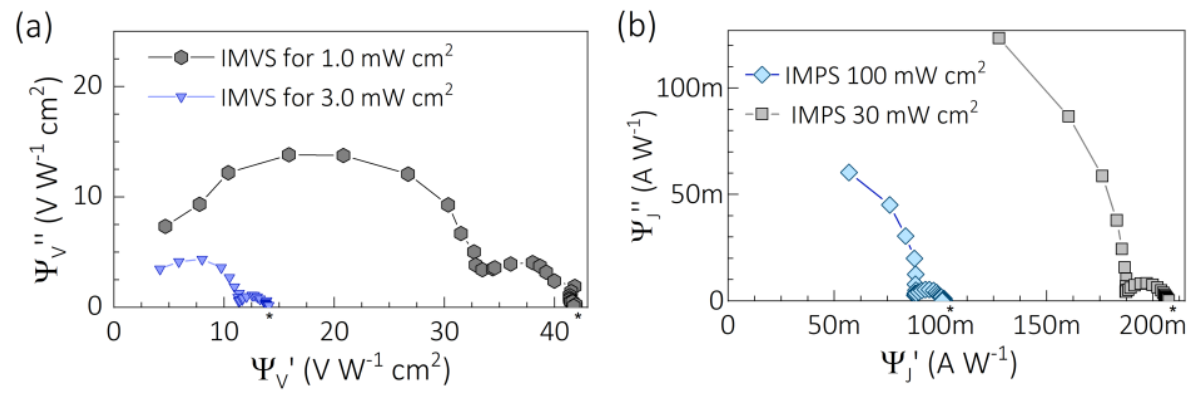

Figure 5.7. Individual (a) IMVS and (b) IMPS spectra of the PPmixP sample (see Table 3.1) at open-circuit under different illumination intensities, as indicated, in the Nyquist plot representation. The asterisks mark the low-frequency limits.

Employing the LIMIS definition (5.11) permits the comparison with the IS spectra, as in Figure 5.8b-f. Similarly to Figure 5.2, two main arcs can be found in the impedance Nyquist plots by IS as well as LIMIS, as in Figure 5.8b. However, a closer inspection at lower frequencies may reveal a third arc which is more evident a higher illumination intensity, as in Figure 5.8c. This new feature in the samples PPmixP, SmixP and SmixIP (see Table 3.1) needed the use of an equivalent circuit like that of Figure 5.8d. The two components of the low frequency branch of the spectra were sometimes not easy to resolve, as in the case of the SmixP sample in the capacitance Bode plot representation of Figure 5.8e. Distinctly, the PPmixP device clearly shows three steps in the capacitance Bode plots of Figure 5.8f. 
110

At the higher frequencies $(f>1 \mathrm{kHz})$, the LIMIS spectra result negative in the Nyquist plot (empty dots). This also produces negative capacitances in the Bode plots of Figure 5.8. This may suggest that further circuit elements should be considered for modeling the high frequency part of the LIMIS spectra. On the other hand, the IS spectra above $1 \mathrm{kHz}$ behave mainly as earlier described. ${ }^{[38-39]}$

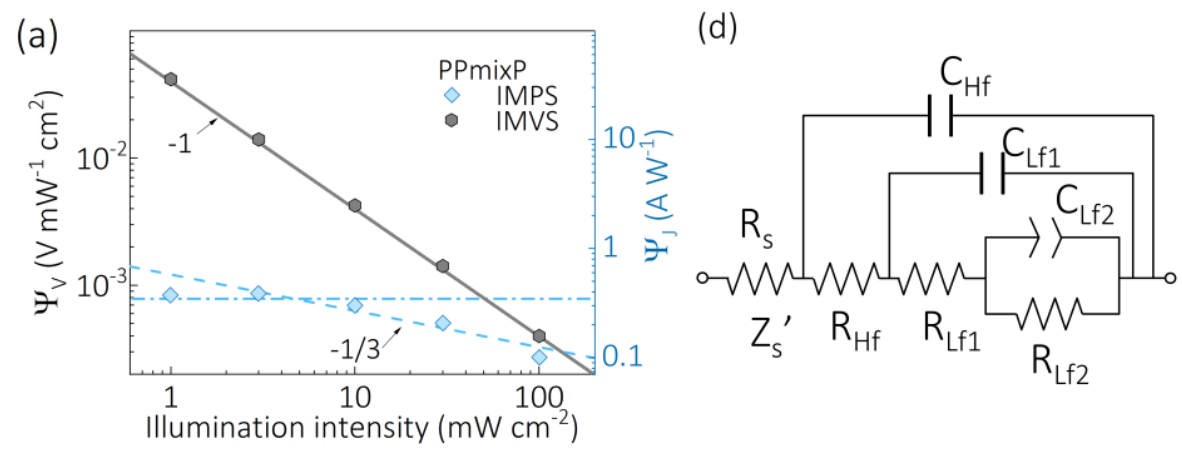

(d)
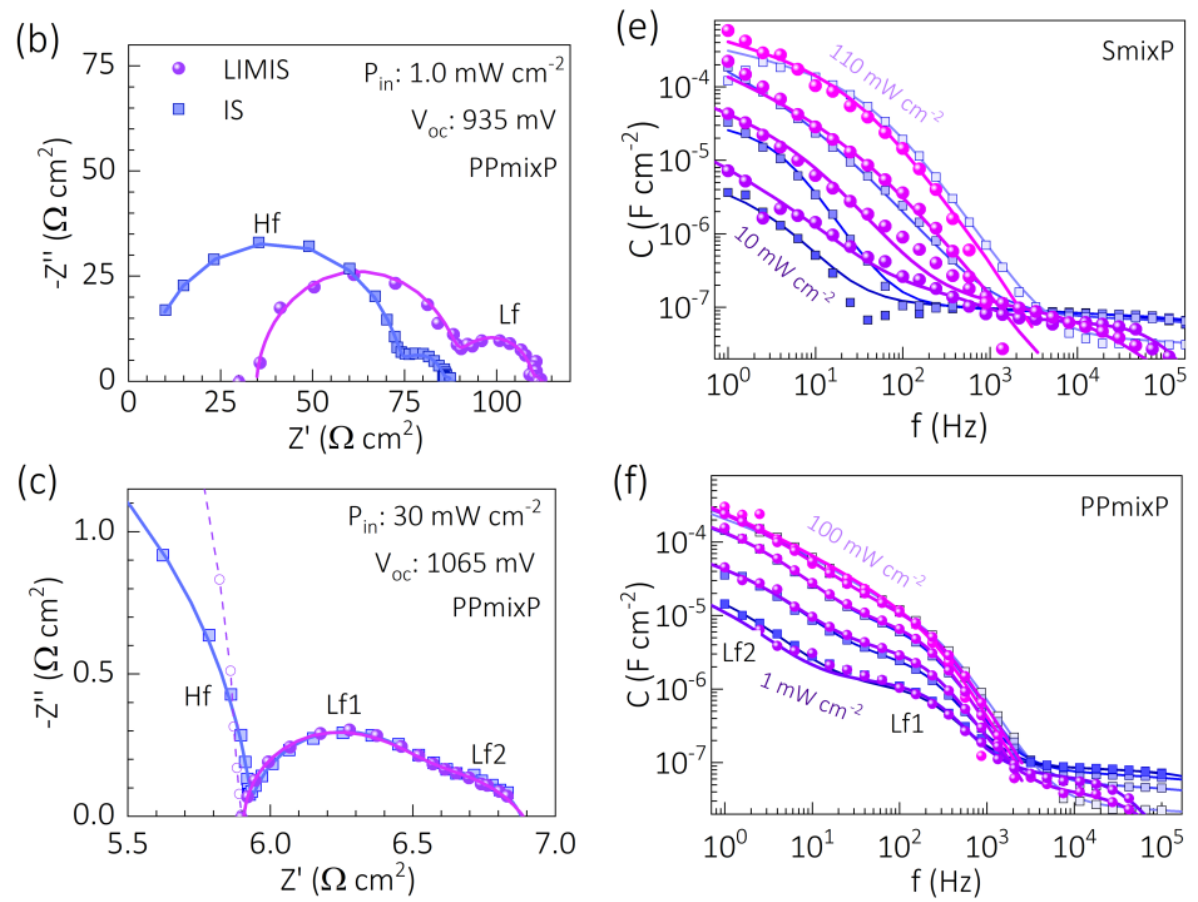

Figure 5.8. LIMIS spectra of PSC at OC under different $\bar{P}_{\text {in }}$ : (a) low frequency limits of IMPV and IMPS; impedance spectra from IS and LIMIS at (b) lower and (c) higher illumination intensities; (d) EC model used in most of the fittings, and capacitance Bode plots comparing IS and LIMIS from two different samples showing (e) one and (f) two low 
frequency components. The sample structures are indicated in Table 3.1. Dots are experimental data and lines are fittings. Open dots in (c) are negative. Arrow-pointed numbers in (a) indicate the power law in each trend.

An extra series resistance-like effect is found for the LIMIS spectra in comparison with the IS spectra. The impedance Nyquist plot is found right shifted, as in the Figure 5.8b, because of more complex high frequency features producing negative arcs crossing the $Z^{\prime}$ axis, as in Figure 5.8c. In the next these effects are parametrized as $Z_{s}{ }^{\prime}$ similarly to a larger $R_{s}$ for the LIMIS spectra, as in Figure 5.8d.

At low frequencies $(f<1 \mathrm{kHz})$ LIMIS and IS spectra seem to behave in good agreement. This more is evident for the PPmixP device in both, the impedance Nyquist plot (Figure 5.8c) and the capacitance Bode plots (Figure 5.8f).

One can compare LIMIS and IS by using $\Delta Z_{\Psi}$ at the low frequency limit of the impedance as $\Delta Z_{\Psi}^{\prime}=\left(Z_{T}{ }^{\prime}-R_{T}\right) / R_{T}$ where total impedance $Z_{\mathrm{T}}^{\prime}$ and total resistance $R_{T}$ come from LIMIS and IS respectively. Preliminary observations of $\Delta Z_{\Psi}^{\prime}$ indicate that the higher $\Delta Z_{\Psi}^{\prime}$ the best, and negative values of $\Delta Z_{\Psi}^{\prime}$ can be correlated with lower performance and/or stability in PSCs.

The EC of Figure 5.8d was the main fitting model for the IS and LIMIS spectra. In some exceptions the EC of Figure 5.2c was alternatively needed, but always neglecting the inductive elements. ${ }^{[2]}$

The low frequency capacitances are compared between LIMIS and IS in Figure 5.9a,b. For the PPmixP sample IS delivers only slightly larger values than LIMIS and an exponential trend $C_{H f 1} \propto \exp \left[3 q V_{o c} / 2 k_{B} T\right]$ is found in Figure 5.9a, similarly to mixed-perovskite cells in Section 5.2.1. ${ }^{[2,39]}$ However, the even higher $C_{H f 2}$ seems to be saturating the exponential trend with $m_{c} \approx$ 5.0 , or just following a linear increase with $V_{o c}$. The idea of a saturating process is probably more evident in the SmixP sample where the IS and LIMIS result only converge at larger illumination intensities/voltages, in Figure 5.9b.

The resistances $R_{T}$ and $R_{S}$ from IS and the analogous $Z_{\mathrm{T}}^{\prime}$ and $Z_{\mathrm{S}}^{\prime}$ from LIMIS are summarized in Figure 5.9c,d. Note that $R_{T}=R_{H f}+R_{L f 1}+R_{L f 2}+R_{S} \approx R_{H f}$ for IS, while $Z_{\mathrm{T}}^{\prime}=R_{H f}+$ $R_{L f 1}+R_{L f 2}+Z_{\mathrm{S}}^{\prime} \approx Z_{\mathrm{S}}^{\prime}$ for LIMIS. Furthermore, the total $C$-coupled resistances in LIMIS include only low frequency contributions $R_{L f} \approx R_{L f 1}+R_{L f 1}$. The general trends of $R_{T}$ and $Z_{\mathrm{T}}^{\prime}$ indicate slightly larger recombination resistances with ideality factors ranging 1.5-1.7 and nearly constant $R_{S}$ in Figure 5.9c,d. Interestingly, $Z_{\mathrm{s}}^{\prime}$ from LIMIS also exhibits an exponential decay trend with ideality-factor-like parameters between 2.0 and 2.5 .

The small characteristic response times from IS, LIMIS and transient photovoltage (TPV) are presented in Figure 5.9e,f. The high frequency-related characteristic times $\tau_{H f}$ follow the trend of the $R_{H f}$ with $m_{\tau} \approx 1.5$ as Equation (5.3). The TPV lifetimes approximately match $\tau_{H f}$, mainly at the lower illumination intensities. However, ar the light fluence increase the three techniques diverge LIMIS and TPV showing the lowest and highest values, respectively.

Larger time constants are found for the slow frequency RC couples from IS and LIMIS, without significant difference among methods. For the PPmixP sample, $\tau_{H f 1}$ and $\tau_{H f 2}$ behave slightly constant and decreasing, respectively, suggesting an eventual convergence around 
112

milliseconds, in Figure 5.9e. For the SmixP sample, $\tau_{H f}$ slightly decrease in Figure 5.9f, similarly to the behavior of $\tau_{H f 2}$ in Figure 5.9e. In both cases, these slower times are around tens of milliseconds, somewhere below the results from the devices including $\mathrm{TiO}_{2}$ and spiro-OMeTAD as electron and hole selective contacts, respectively.
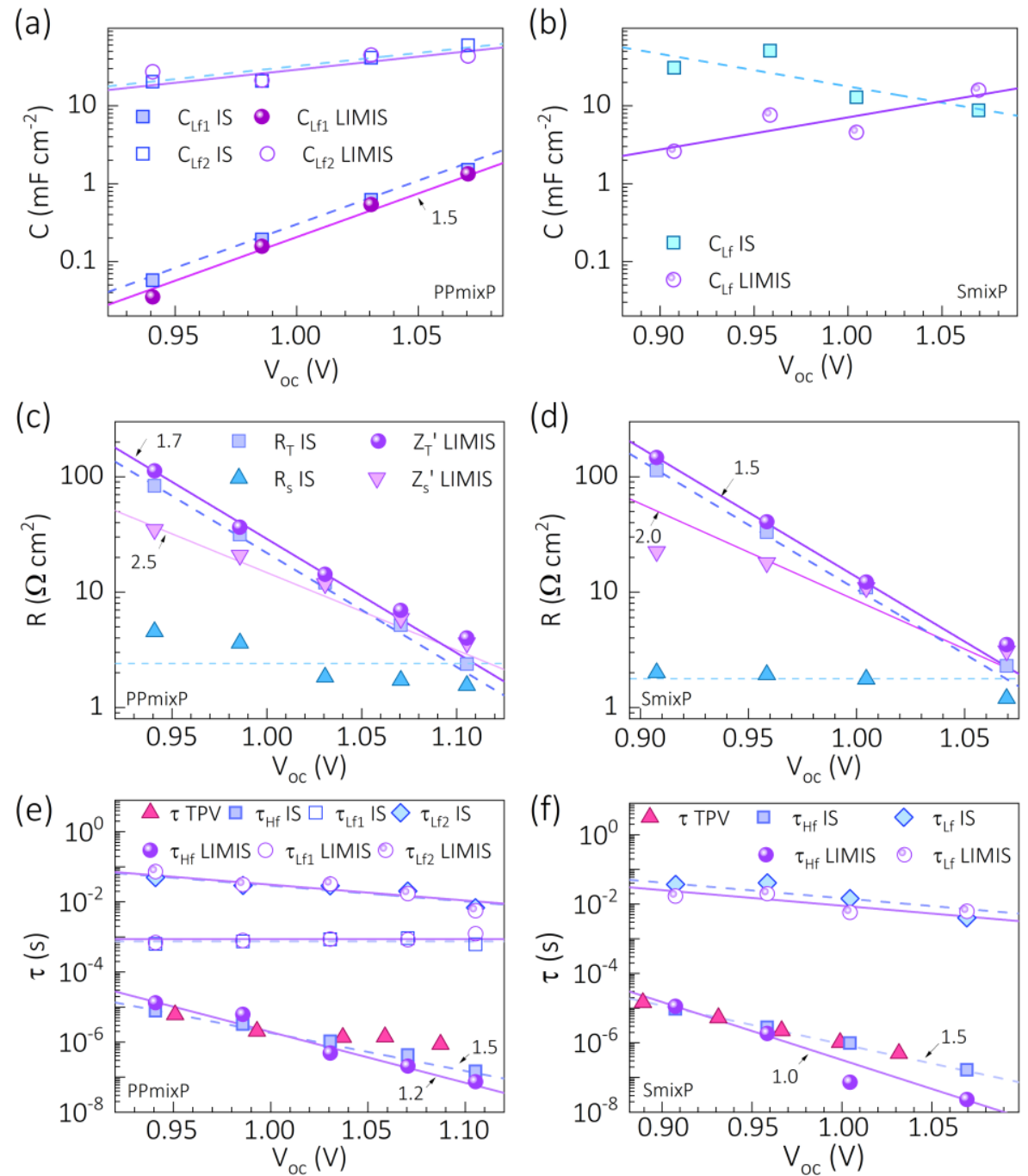

Figure 5.9. Parameters from equivalent circuit modeling of IS and LIMIS spectra of PSCs at OC under different $\bar{P}_{i n}$ : (a, b) capacitance, (c, d) resistance, and (e, f) characteristic response times from PPmixP and SmixP samples, respectively (see Table 3.1). Dots are the 
fitting parameters and lines are exponential/linear fittings highlighting the general trends. Arrow-pointed numbers indicate the $k_{B} T$ coefficient in each exponential trend.

The differences between IS and LIMIS, specifically in terms of lifetimes, make us wonder which one is "the right one" and/or what effects are considered in one method differently to the other. In this direction, a differential analysis can clarify, at least, the issue related with the lifetimes.

Hence, we first introduce the directional derivative definitions for each one of the transfer functions involved in the IS versus LIMIS approach:

$$
\begin{aligned}
& Z=\left(\frac{\partial J}{\partial V}\right)^{-1} \\
& \Psi_{J}=\frac{\partial J}{\partial P_{i n}} \\
& \Psi_{V}=\frac{\partial V_{o c}}{\partial P_{i n}} \\
& Z_{\Psi}=\frac{\partial V}{\partial P_{\text {in }}}\left(\frac{\partial J}{\partial P_{\text {in }}}\right)^{-1}
\end{aligned}
$$

In IS and LIMIS, these magnitudes are obtained spectroscopically, and using EC models or solving the transport equations one gets the total differential resistance and the capacitance, as definitions (2.24) and (2.34) respectively. Traditionally, only IS is used and the resistance is obtained from (5.23a). However, with the information from LIMIS, one can rewrite the total differential resistance as

$$
R=\left(\frac{d J}{d V}\right)^{-1}=\left(\frac{\partial J}{\partial V}+\frac{\partial J}{\partial P_{i n}} \frac{\partial P_{i n}}{\partial V}\right)^{-1}
$$

with a spectroscopic form

$$
R(\omega)=\left(\frac{1}{\operatorname{Re}[Z(\omega)]}+\frac{1}{\operatorname{Re}\left[Z_{\Psi}(\omega)\right]}\right)^{-1}=\left(\frac{1}{R_{I S}(\omega)}+\frac{1}{R_{\Psi}(\omega)}\right)^{-1}
$$

being $R_{I S}$ and $R_{\Psi}$ the resistances form IS and LIMIS, respectively.

Similarly, the total differential capacitance can be obtained as

$$
C=\frac{d Q}{d V}=\frac{\partial Q}{\partial V}+\frac{\partial Q}{\partial P_{i n}} \frac{\partial P_{i n}}{\partial V}
$$

with a frequency dependency

$$
C(\omega)=\operatorname{Re}\left[\frac{1}{i \omega Z(\omega)}+\frac{1}{i \omega Z_{\Psi}(\omega)}\right]=C_{I S}(\omega)+C_{\Psi}(\omega)
$$

Finally, the lifetimes can be found from the product of (5.25) and (5.27), which corrects any estimation by a single method. For instance, if $Z=Z_{\Psi}$ then $R=R_{I S} / 2=R_{\Psi} / 2$ and $C=2 C_{I S}=$ $2 C_{\Psi}$, which makes no changes in the characteristic response times $\tau=R C=R_{I S} C_{I S}=R_{\Psi} C_{I S}$. 
Therefore, the report of $Z \neq Z_{\Psi}$ in PSCs suggest considering lifetime within the values reported by IS and LIMIS.

In summary, LIMIS reproduces most of the general trends and spectra shapes already found by IS, mainly at low frequencies and illumination intensities. However, LIMIS is able to resolve faster processes as illumination intensity increases. Moreover, the comparison between IS and LIMIS corrects the estimation of lifetimes and may be used in future analyses as a figure of merit for characterizing solar cells. In addition, several components in the low frequency capacitance have been found for devices including mixed perovskites as absorber, and $\mathrm{SnO}_{2}$ and PDCBT in the selective contact structures.

\section{References}

[1] O. Almora et al., Sol. Energy Mater. Sol. Cells 195 (2019), 291.

https://doi.org/10.1016/j.solmat.2019.03.003

[2] O. Almora et al., Nano Energy 48 (2018), 63. https://doi.org/10.1016/j.nanoen.2018.03.042

[3] O. Almora, D. Miravet, G. J. Matt, G. Garcia-Belmonte, C. J. Brabec, Appl. Phys. Lett. 116 (2020), 013901. https://doi.org/10.1063/1.5139571

[4] O. Almora et al., Nano Energy submitted (2020), https://arxiv.org/abs/1911.05440.

[5] H.-S. Kim, N.-G. Park, J. Phys. Chem. Lett. 5 (2014), 2927. https://doi.org/10.1021/jz501392m

[6] W. Tress, N. Marinova, T. Moehl, S. M. Zakeeruddin, M. K. Nazeeruddin, M. Gratzel, Energy

Environ. Sci. 8 (2015), 995. https://doi.org/10.1039/C4EE03664F

[7] Y. Zou, R. J. Holmes, Adv. Energy Mater. 6 (2016), 1501994.

https://doi.org/10.1002/aenm.201501994

[8] G. A. Nemnes et al., Sol. Energy Mater. Sol. Cells 159 (2017), 197.

https://doi.org/10.1016/j.solmat.2016.09.012

[9] P. Lopez-Varo et al., Adv. Energy Mater. 8 (2018), 1702772.

http://onlinelibrary.wiley.com/doi/10.1002/aenm.201702772/full

https://doi.org/10.1002/aenm.201702772

[10] A. Guerrero et al., Adv. Energy Mater. 8 (2018), 201703376.

https://doi.org/10.1002/aenm.201703376

[11] W. Tress et al., Energy Environ. Sci. 11 (2017), 151. https://doi.org/10.1039/C7EE02415K

[12] G. Richardson et al., Energy Environ. Sci. 9 (2016), 1476. https://doi.org/10.1039/C5EE02740C

[13] S. Ravishankar et al., J. Phys. Chem. Lett. 8 (2017), 915.

http://dx.doi.org/10.1021/acs.jpclett.7b00045

[14] H. Shen et al., J. Phys. Chem. Lett. 8 (2017), 2672. https://doi.org/10.1021/acs.jpclett.7b00571

[15] R. A. Belisle et al., Energy Environ. Sci. 10 (2017), 192. https://doi.org/10.1039/C6EE02914K

[16] O. Almora, C. Aranda, I. Zarazua, A. Guerrero, G. Garcia-Belmonte, ACS Energy Lett. 1 (2016), 209. https://doi.org/10.1021/acsenergylett.6b00116

[17] O. Almora, L. Vaillant-Roca, G. Garcia-Belmonte, Rev. Cubana Fis. 34 (2017), 58.

http://www.revistacubanadefisica.org/index.php/rcf/article/view/RCF_34-1_58

[18] Y. Rong et al., Energy Environ. Sci. 10 (2017), 2383. https://doi.org/10.1039/C7EE02048A

[19] D.-H. Kang, N.-G. Park, Adv. Mater. 31 (2019), 1805214. https://doi.org/10.1002/adma.201805214

[20] N. Onoda-Yamamuro, T. Matsuo, H. Suga, J. Phys. Chem. Solids 53 (1992), 935.

https://doi.org/10.1016/0022-3697(92)90121-S

[21] Jarvist M. Frost, Keith T. Butler, Federico Brivio, Christopher H. Hendon, Mark van Schilfgaarde,

A. Walsh, Nano Lett. 14 (2014), 2584. https://doi.org/10.1021/nl500390f

[22] M. Sendner et al., Mater. Horiz. 3 (2016), 613. https://doi.org/10.1039/C6MH00275G

[23] O. Almora, A. Guerrero, G. Garcia-Belmonte, Appl. Phys. Lett. 108 (2016), 043903.

https://doi.org/10.1063/1.4941033

[24] P. Lopez-Varo et al., ACS Energy Lett. 2 (2017), 1450.

https://doi.org/10.1021/acsenergylett.7b00424

[25] M. García-Rosell, A. Bou, J. A. Jiménez-Tejada, J. Bisquert, P. Lopez-Varo, J. Phys. Chem. C 122 (2018), 13920. https://doi.org/10.1021/acs.jpcc.8b01070 
[26] C. Bat-El, W. Małgorzata, E. Lioz, Adv. Funct. Mater. 27 (2017), 1604733.

https://doi.org/10.1002/adfm.201604733

[27] E. A. Muljarov, S. G. Tikhodeev, N. A. Gippius, T. Ishihara, Phys. Rev. B 51 (1995), 14370. https://doi.org/10.1103/PhysRevB.51.14370

[28] I. C. Smith, E. T. Hoke, D. Solis-Ibarra, M. D. McGehee, H. I. Karunadasa, Angew. Chem. 126 (2014), 11414. https://doi.org/10.1002/ange.201406466

[29] J.-W. Lee et al., Nat. Commun. 9 (2018), 3021. https://doi.org/10.1038/s41467-018-05454-4

[30] G. A. Nemnes et al., J. Phys. Chem. C 121 (2017), 11207. https://doi.org/10.1021/acs.jpcc.7b04248

[31] I. Zarazua et al., J. Phys. Chem. Lett. 7 (2016), 5105. https://doi.org/10.1021/acs.jpclett.6b02193

[32] A. Guerrero et al., J. Phys. Chem. C 120 (2016), 8023. https://doi.org/10.1021/acs.jpcc.6b01728

[33] A. Todinova et al., ChemElectroChem 4 (2017), 1. https://doi.org/10.1002/celc.201700498

[34] O. Almora, I. Zarazua, E. Mas-Marza, I. Mora-Sero, J. Bisquert, G. Garcia-Belmonte, J. Phys. Chem. Lett. 6 (2015), 1645. https://doi.org/10.1021/acs.jpclett.5b00480

[35] E. Ghahremanirad, A. Bou, S. Olyaee, J. Bisquert, J. Phy. Chem. Lett. 8 (2017), 1402. https://doi.org/10.1021/acs.jpclett.7b00415

[36] A. Kovalenko, J. Pospisil, J. Krajcovic, M. Weiter, A. Guerrero, G. Garcia-Belmonte, Appl. Phys. Lett. 111 (2017), 163504. https://doi.org/10.1063/1.4990788

[37] I. Zarazua, J. Bisquert, G. Garcia-Belmonte, J. Phys. Chem. Lett. 7 (2016), 525.

https://doi.org/10.1021/acs.jpclett.5b02810

[38] O. Almora, C. Aranda, G. Garcia-Belmonte, J. Phys. Chem. C 122 (2018), 13450.

https://doi.org/10.1021/acs.jpcc.7b11703

[39] O. Almora, G. Garcia-Belmonte, Solar Energy 189 (2019), 103.

https://doi.org/10.1016/j.solener.2019.07.048

[40] E. Menéndez-Proupin, P. Palacios, P. Wahnón, J. C. Conesa, Phys. Rev. B 90 (2014), 045207. https://doi.org/10.1103/PhysRevB.90.045207

[41] G. Giorgi, J.-I. Fujisawa, H. Segawa, K. Yamashita, J. Phys. Chem. Lett. 4 (2013), 4213. https://doi.org/10.1021/jz4023865

[42] J. Bisquert, G. Garcia-Belmonte, I. Mora-Sero, in Unconventional Thin Film Photovoltaics, (Eds: E. Da Como, F. De Angelis, H. J. Snaith, A. Walker), The Royal Society of Chemistry, Cambridge 2016, 57. http://dx.doi.org/10.1039/9781782624066-00057

[43] J. S. Manser, P. V. Kamat, Nat. Photonics 8 (2014), 737. http://dx.doi.org/10.1038/nphoton.2014.171

[44] D. A. Jacobs et al., J. Appl. Phys. 124 (2018), 225702. https://doi.org/10.1063/1.5063259

[45] D. Moia et al., Energy Environm. Sci. 12 (2019), 1296. https://doi.org/10.1039/C8EE02362J

[46] F. Ebadi, N. Taghavinia, R. Mohammadpour, A. Hagfeldt, W. Tress, Nat. Commun. 10 (2019), 1574. https://doi.org/10.1038/s41467-019-09079-z

[47] E. J. Juarez-Perez et al., J. Phys. Chem. Lett. 5 (2014), 2390. https://doi.org/10.1021/jz5011169

[48] T.-Y. Yang, G. Gregori, N. Pellet, M. Grätzel, J. Maier, Angew. Chem. Int. Ed. 54 (2015), 7905. https://doi.org/10.1002/anie.201500014

[49] H.-S. Kim et al., J. Phys. Chem. Lett. 6 (2015), 4633. https://doi.org/10.1021/acs.jpclett.5b02273

[50] L. Li, F. Wang, X. Wu, H. Yu, S. Zhou, N. Zhao, J. Phys. Chem. C 120 (2016), 2536. https://doi.org/10.1021/acs.jpcc.5b11627

[51] Y.-C. Zhao, W.-K. Zhou, X. Zhou, K.-H. Liu, D.-P. Yu, Q. Zhao, Light: Sci. Appl. 6 (2017), e16243. https://doi.org/10.1038/lsa.2016.243

[52] J. Xing, Q. Wang, Q. Dong, Y. Yuan, Y. Fang, J. Huang, Phys. Chem. Chem. Phys. 18 (2016), 30484. https://doi.org/10.1039/C6CP06496E

[53] X. Tang, G. J. Matt, S. Gao, E. Gu, O. Almora, C. J. Brabec, ACS Appl. Mater. Interfaces 11 (2019), 39018. https://doi.org/10.1021/acsami.9b14788

[54] O. Almora, L. G. Gerling, C. Voz, R. Alcubilla, J. Puigdollers, G. Garcia-Belmonte, Sol. Energy Mater. Sol. Cells 168 (2017), 221. https://doi.org/10.1016/j.solmat.2017.04.042

[55] O. Almora, C. Aranda, E. Mas-Marzá, G. Garcia-Belmonte, Appl. Phys. Lett. 109 (2016), 173903. https://doi.org/10.1063/1.4966127

[56] O. Almora, M. García-Batlle, G. Garcia-Belmonte, J. Phys. Chem. Lett. 10 (2019), 3661. https://doi.org/10.1021/acs.jpclett.9b00601

[57] J. Bisquert, Phys. Chem. Chem. Phys. 5 (2003), 5360. https://doi.org/10.1039/B310907K 
[58] Y. T. Set, E. Birgersson, J. Luther, Phys. Rev. Appl. 5 (2016), 054002. https://doi.org/10.1103/PhysRevApplied.5.054002

[59] J. A. Anta et al., Phys. Chem. Chem. Phys. 14 (2012), 10285. https://doi.org/10.1039/C2CP40719A

[60] E. Kamieniecki, J. Vac. Sci. Technol. 20 (1982), 811. https://doi.org/10.1116/1.571491

[61] E. Kamieniecki, J. Appl. Phys. 54 (1983), 6481. https://doi.org/10.1063/1.331876

[62] R. Peat, L. M. Peter, J. Electroanal. Chem. Interfacial Electrochem. 228 (1987), 351. https://doi.org/10.1016/0022-0728(87)80117-1

[63] E. A. Ponomarev, L. M. Peter, J. Electroanal. Chem. 396 (1995), 219. https://doi.org/10.1016/00220728(95)04115-5

[64] L. Dloczik et al., J. Phys. Chem. B 101 (1997), 10281. https://doi.org/10.1021/jp972466i

[65] P. E. de Jongh, D. Vanmaekelbergh, J. Phys. Chem. B 101 (1997), 2716.

https://doi.org/10.1021/jp962226n

[66] G.-O. Kim, Bull. Korean Chem. Soc. 33 (2012), 469. https://doi.org/10.5012/BKCS.2012.33.2.469

[67] A. Pockett et al., J. Phys. Chem. C 119 (2015), 3456. https://doi.org/10.1021/jp510837q

[68] Y. Gao, A. J. Wise, A. K. Thomas, J. K. Grey, ACS Appl. Mater. Interfaces 8 (2016), 285. https://doi.org/10.1021/acsami.5b08724

[69] L. Bertoluzzi, J. Bisquert, J. Phys. Chem. Lett. 8 (2017), 172.

https://doi.org/10.1021/acs.jpclett.6b02714

[70] S. Ravishankar, C. Aranda, S. Sanchez, J. Bisquert, M. Saliba, G. Garcia-Belmonte, J. Phys. Chem. C 123 (2019), 6444. https://doi.org/10.1021/acs.jpcc.9b01187

[71] S. Ravishankar, A. Riquelme, S. K. Sarkar, M. Garcia-Batlle, G. Garcia-Belmonte, J. Bisquert, J. Phys. Chem. C 123 (2019), 24995. https://doi.org/10.1021/acs.jpcc.9b07434

[72] J. Krüger, R. Plass, M. Grätzel, P. J. Cameron, L. M. Peter, J. Phys. Chem. B 107 (2003), 7536. https://doi.org/10.1021/jp0348777

[73] Y. T. Set, B. Li, F. J. Lim, E. Birgersson, J. Luther, Appl. Phys. Lett. 107 (2015), 173301. https://doi.org/10.1063/1.4934920

[74] X. Chen, Y. Shirai, M. Yanagida, K. Miyano, Phys. Chem. Chem. Phys. 20 (2018), 17918. https://doi.org/10.1039/C8CP01227J

[75] H. Song, D. D. Macdonald, J. Electrochem. Soc. 138 (1991), 1408.

https://doi.org/10.1149/1.2085796

[76] J. Halme, Phys. Chem. Chem. Phys. 13 (2011), 12435. https://doi.org/10.1039/C1CP21134J

[77] J. Bisquert, F. Fabregat-Santiago, in Dye-sensitized solar cells, (Ed: K. Kalyanasundaram), CRC

Press, Lausanne (Switzerland) 2010, 457.

[78] C. A. Schiller, R. Kaus, ECS Transactions 25 (2010), 49. https://doi.org/10.1149/1.3324743 


\section{Chapter 6. Conclusions}

The study of the capacitance in photovoltaic devices allows to discern: (i) the charge carrier profiles defining the p-n junction-like contact selectivity structure, (ii) the deep levels defect distribution related with the SRH recombination mechanisms and (iii) informs on the carriers lifetimes which characterizes general recombination and charge collection mechanisms. These studies are typically based on impedance spectroscopy (IS) analyses, that (iv) could be also used to characterize materials in terms of their dielectric and conductivity responses.

The studies on the bulk properties of $\mathrm{CH}_{3} \mathrm{NH}_{3} \mathrm{PbI}_{3}$ (MAPI) pellet samples suggested that hopping conductivities and electrode polarization processes could be occurring. The presence of space charge regions in symmetric contacted samples resulted in large temperature activated lowfrequency capacitances from IS measurements and slow charging kinetics in the Sawyer-Tower circuit experiments.

The dark characterization of perovskite solar cells (PSCs) showed the presence of time evolving modifications of the current-voltage characteristic depending on the measurement conditions, named $J-V$ curve hysteresis, and large low-frequency capacitances from the IS experiments. The dark $J-V$ curves evidenced two main forms of hysteretic currents: capacitive and non-capacitive.

The dark capacitive hysteretic currents up to tens of $\mu \mathrm{A} \cdot \mathrm{cm}^{-2}$ scaled with the voltage scan rate at voltages below the flat-band condition and were discarded to be related with the dielectric response. The formation of space charge regions towards the interfaces seems to be the mechanism behind these phenomena, which also produce the low-frequency capacitance increase. Importantly, the ionic contribution to the low-frequency capacitance from IS is thermally activated and can hinder the evaluation of deep level trap defect concentrations. Thus, only frequencies above $1 \mathrm{kHz}$ should be taken for thermal admittance spectroscopy studies in PSCs. These capacitive features were found nearly universal for all perovskite-based devices and samples. Interestingly, the use of organic selective contacts in inverted configurations can modify the time scale of these capacitive currents due to resistive artifacts. Besides, the selective contacts, e.g. spiroOMeTAD, can contribute significantly to the hysteretic currents and the capacitance spectra

The non-capacitive hysteretic currents were found to increase as the time of polarization around or above the flat-band regime. Here Faradaic processes cannot be discarded, but most likely the reordering of mobile ions may be the main mechanism to consider. This hysteretic feature is the most dynamic and unreproducible in terms of shapes and/or scales, but also universal in PSCs.

The redistribution of mobile ions in space charge regions has the particular effect of distorting the capacitance as a function of DC voltage in the Mott-Schottky analyses. The perovskites with applications in photovoltaics are significantly intrinsic (excepting some tin- and chloride-based perovskites) which gives a p-i-n junction character to the PSCs. However, instead of typical p-i$n$ junction-like patterns, space charge regions of the mobile ions can effectively shrink the intrinsic region width producing more $p-n$ junction like patterns. In any case, care must be taken with the interpretation of doping densities and built-in voltages from the Mott-Schottky plots in PSCs. 


\section{8}

The light hysteresis was characterized in the named "dipolar switching" experiment. The devices were set under 1 sun illumination intensity and fast scan rates were measured after a minute of pre-bias. As result, the short-circuit current and the open circuit voltage varied tens of $\mathrm{mA} \cdot \mathrm{cm}^{-}$ 2 and hundreds of $\mathrm{mV}$, respectively, forming patterns of normal and inverted hysteresis in devices comprising MAPI and mixed perovskites, respectively. Drift diffusion simulations showed these experimental results responding to the formation of ionic dipoles which can or cannot switch when normal hysteresis or inverted hysteresis, in MAPI- or mixed perovskite-based devices, respectively. Importantly, the modification of interfaces by including 2D capping layers resulted in the largest hysteretic currents for both the normal and inverted hysteresis, for MAPI- and mixed perovskite-based devices respectively. This indicates that the control of the ionic dipoles may require the balanced optimization between the interfaces and the absorber layer.

The light IS analysis at open circuit (OC) under different illumination intensities showed two main regimes of resistive and capacitive features: towards lower and higher frequencies. At lower light intensities, the high and low frequencies resistances can be identified with bulk and interface leakage current recombination resistances. At higher illumination intensities, the resistances follow similar exponential trends, informing on the ideality factor $m$, and thus the main recombination mechanism. For MAPI devices $m \approx 2$ suggest a major importance of SRH recombination and/or interface phenomena, while in mixed perovskite cells $m \approx 1.5$, possibly indicating larger influence of band-to-band radiative recombination and/or diffusion processes. On the other hand, capacitances resulted nearly constant at higher frequencies and exponentially increasing at lower frequencies, up to tens of $\mathrm{mF} \cdot \mathrm{cm}^{-2}$. Interestingly, for the most optimized devices including mixed perovskite solar cells with up to $20 \%$ efficiency the bulk related features seem to be predominant in the electrical response. This indicates that after the interface optimization the bulk absorber quality may be the most important element to consider in order to increase performance in PSCs.

The low frequency capacitance is significantly large in PSCs at OC, short-circuit (SC), and dark forward bias above flat-band condition. In all these cases the increase of charge carrier concentration and/or photon fluence seems to enhance the slow kinetics phenomena producing larger low-frequency capacitances and heavier distortions in the $J-V$ curve, i.e. large hysteresis. Then one can consider a proportionality between the charge carrier concentrations and photon fluences with the ionization and/or activation of the mobile ions. Assuming such proportionality allows to explain the large light low frequency capacitance at SC as sue to the AC response of space charge regions of mobile ions. Additionally, at OC the constant and equally distributed mobile anions and cations can produce an ionic chemical capacitance. The latter may respond for the largest and slowest contribution to the capacitance spectra in PSCs.

Finally, a new concept for the AC characterization of photovoltaic devices at OC was introduced: the light intensity modulated impedance spectroscopy (LIMIS). By combining the intensity modulated photocurrent and photovoltage spectroscopies, IMPS and IMVS respectively, the photoimpedance LIMIS=IMVS/IMPS is obtained due to small light perturbation, as an alternative to the bias perturbation. The difference between impedances from IS and LIMIS is showed to inform on the recombination velocity in devices with the traditional $\mathrm{p}-\mathrm{n}^{+}$junction. Preliminary characterization in PSCs show general agreement between IS and LIMIS at lower illumination 
intensities and larger differences at higher light intensities, suggesting a correction to the lifetimes.

Outlook: The main conclusions of this work suggest that the anomalous hysteresis and lowfrequency capacitive features of PSCs are due to the slow kinetics of mobile ions. First, this indicate that every characterization technique may revise their formalism in order to consider these effects and avoid "fake" conclusions. Second, the influence of these mobile ions on the overall performance of PSCs should be further clarified. And third, the potential of these features for other applications should be explored. Furthermore, the results herein presented are also starting points for future investigations. For instance, the large photo-induced capacitance in PSCs could contribute to energy-storage units in hybrid or stand-alone photovoltaic systems (PVSs) and/or serve as stabilizers for electricity supply to the net in hybrid or grid-connected PVSs.

The control of conductivity in photovoltaic solar cells is a very attractive open problem. In this regard, it would be interesting to explore the introduction of extrinsic dopants and/or the control of crystallinity. For instance, the development of effective acceptor perovskites may allow to prescind of the hole transport layer, which could even improve the device stability. In this direction, tone could explore the combination and/or substitution of solution processed fabrication methods with/for others, like vapor deposition.

Besides the devices, the material characterizations of MAPI and newer mixed photovoltaic perovskites are still pending to clarify the electronic/ionic conductivity and polarization mechanisms. Several experiments varying composition, temperature, light intensity and wavelength could provide better understanding and more argument for newer theories. Purposely, the scattering in the estimation of the static dielectric constant from first principle methods is still under debate.

Some combinations of IS experiments in PSCs as a function of temperature, illumination and DC bias are still lacking. More importantly here, it is the determination of the nature of the mobile ions and the respective activation/ionization energies. Identifying these mobile ions impact more fundamental research on the stability of PSCs. The IS has the potential to become the effective evaluation method for studying the ionic concentrations in perovskite-based devices. Moreover, the combination of first principles and drift-diffusion simulations are possibly the best strategies.

The concept of LIMIS is still in early phases of development. Experimentally, the study of LIMIS as a function of temperature and illumination wavelength are the clearer next steps. Theoretically, more realistic drift-diffusion analyses may be conducted aiming to simulate the several device structures and experimental spectra. The goal would be to elaborate methodologies by combining the use of IS and LIMIS in identifying characteristic bulk and/or interface recombination mechanism. In this regard, one crucial element would be to use machine learning algorithms to overcome the main issue behind the use of IS, IMPS, IMVS or LIMIS techniques: the timeconsuming task of processing, presenting and modeling impedance spectra via equivalent circuits. In addition, similarly to LIMIS, new approaches can be proposed by studying the photovoltage and photocurrent responses under the same DC conditions, but under an small AC perturbation, e.g. magnetic. 


\section{Annex A: List of publications included in this work}

1. O. Almora, A. Guerrero, G. Garcia-Belmonte*, Ionic Charging by Local Imbalance at Interfaces in Hybrid Lead Halide Perovskites, Appl. Phys. Lett. 108 (2016), https://doi.org/043903. 10.1063/1.4941033

2. O. Almora, I. Zarazua, E. Mas-Marza, I. Mora-Sero, J. Bisquert, G. Garcia-Belmonte*, Capacitive Dark Currents, Hysteresis, and Electrode Polarization in Lead Halide Perovskite Solar Cells, J. Phys. Chem. Lett. 6 (2015), 1645. https://doi.org/10.1021/acs.jpclett.5b00480

3. O. Almora, C. Aranda, E. Mas-Marzá, G. Garcia-Belmonte*, On Mott-Schottky Analysis Interpretation of Capacitance Measurements in Organometal Perovskite Solar Cells, Appl. Phys. Lett. $109 \quad$ (2016), 173903. https://doi.org/10.1063/1.4966127

4. O. Almora, C. Aranda, I. Zarazua, A. Guerrero, G. Garcia-Belmonte*, Noncapacitive Hysteresis in Perovskite Solar Cells at Room Temperature, ACS Energy Lett. 1 (2016), 209. https://doi.org/10.1021/acsenergylett.6b00116

5. O. Almora, C. Aranda, G. Garcia-Belmonte, Do Capacitance Measurements Reveal Light-Induced Bulk Dielectric Changes in Photovoltaic Perovskites?, J. Phys. Chem. C 122 (2018), 13450. https://doi.org/10.1021/acs.jpcc.7b11703

6. O. Almora*, K.T. Cho, S. Aghazada, I. Zimmermann, G.J. Matt, C.J. Brabec, M.K. Nazeeruddin, G. Garcia-Belmonte*, Discerning Recombination Mechanisms and Ideality Factors through Impedance Analysis of High-Efficiency Perovskite Solar Cells, Nano Energy 48 (2018), 63. https://doi.org/10.1016/j.nanoen.2018.03.042

7. O. Almora*, M. García-Batlle, G. Garcia-Belmonte*, Utilization of TemperatureSweeping Capacitive Techniques to Evaluate Band Gap Defect Densities in Photovoltaic Perovskites, J. Phys. Chem. Lett. $10 \quad$ (2019), 3661. https://doi.org/10.1021/acs.jpclett.9b00601

8. O. Almora, G. Garcia-Belmonte*, Light capacitances in silicon and perovskite solar cells, Solar Energy 189 (2019), 103. https://doi.org/10.1016/j.solener.2019.07.048

9. O. Almora*, P. Lopez-Varo, K.T. Cho, S. Aghazada, W. Meng, Y. Hou, C. Echeverría-Arrondo, I. Zimmermann, G.J. Matt, J.A. Jiménez-Tejada, C.J. Brabec, M.K. Nazeeruddin, G. Garcia-Belmonte*, Ionic Dipolar Switching Hinders Charge Collection in Perovskite Solar Cells with Normal and Inverted Hysteresis, Sol. Energy Mater. Sol. Cells 195 (2019), 291. https://doi.org/10.1016/j.solmat.2019.03.003

10. O. Almora*, D. Miravet, G.J. Matt, G. Garcia-Belmonte, C.J. Brabec, Analytical Model for Light Modulating Impedance Spectroscopy (LIMIS) in All-Solid-State p-n Junction Solar Cells at Open-Circuit, Appl. Phys. Lett. 116 (2020), 013901. https://doi.org/10.1063/1.5139571 


\section{Annex B: List of other publications by the author}

1. O. Almora, L.G. Gerling, C. Voz, R. Alcubilla, J. Puigdollers*, G. Garcia-Belmonte*, Superior Performance of $\mathrm{V}_{2} \mathrm{O}_{5}$ as Hole Selective Contact over other Transition Metal Oxides in Silicon Heterojunction Solar Cells, Sol. Energy Mater. Sol. Cells 168 (2017), 221. https://doi.org/10.1016/j.solmat.2017.04.042

2. O. Almora*, L. Vaillant-Roca, G. Garcia-Belmonte, Perovskite Solar Cells: A brief Introduction and some Remarks, Rev. Cubana Fis. 34 (2017), 58.

3. O. Almora, L. Vaillant-Roca*, A. Bosio, Electrical Characterizations of CdTe/CdS Poly-Crystalline Thin Film Solar Cells, Rev. Cubana Fis. 31 (2014), 66.

4. O. Almora, L. Vaillant-Roca*, J.C. Rimada-Herrera, A. Bosio, DLTS Measurements on CdTe/CdS Polycrystalline Thin Films Solar Cells, Rev. Cubana Fis. 31 (2014), E87.

5. P. Lopez-Varo, J.A. Jiménez-Tejada, M. García-Rosell, S. Ravishankar, G. GarciaBelmonte, J. Bisquert*, O. Almora*, Device Physics of Hybrid Perovskite Solar Cells: Theory and Experiment, Adv. Energy Mater. 8 (2018), 1702772. https://doi.org/10.1002/aenm.201702772

6. H. Bechert*, O. Almora*, K. Regau, G.J. Matt, C.J. Brabec, T. Wehlus, Thin-Film Electrostatic Discharge Protection for Highly Segmented OLEDs in Automotive Applications, Adv. Mater. Technol. 4 (2019), 1800696. https://doi.org/10.1002/admt.201800696

7. M. Coll*, A. Gomez, E. Mas-Marza, O. Almora, G. Garcia-Belmonte, M. CampoyQuiles, J. Bisquert, Polarization Switching and Light-Enhanced Piezoelectricity in Lead Halide Perovskites, J. Phys. Chem. Lett. 6 (2015), 1408. https://doi.org/10.1021/acs.jpclett.5b00502

8. J. Carrillo, A. Guerrero, S. Rahimnejad, O. Almora, I. Zarazua, E. Mas-Marza, J. Bisquert, G. Garcia-Belmonte*, Ionic Reactivity at Contacts and Aging of Methylammonium Lead Triiodide Perovskite Solar Cells, Adv. Energy Mater. 6 (2016), 1502246 https://doi.org/10.1002/aenm.201502246

9. M. Valles-Pelarda, B.C. Hames, I. García-Benito, O. Almora, A. Molina-Ontoria, R.S. Sánchez, G. Garcia-Belmonte, N. Martín*, I. Mora-Sero*, Analysis of the Hysteresis Behavior of Perovskite Solar Cells with Interfacial Fullerene Self-Assembled Monolayers, J. Phys. Chem. Lett. 7 (2016), 4622. https://doi.org/10.1021/acs.jpclett.6b02103

10. S. Ravishankar, O. Almora, C. Echeverría-Arrondo, E. Ghahremanirad, C. Aranda, A. Guerrero, F. Fabregat-Santiago, A. Zaban, G. Garcia-Belmonte, J. Bisquert, Surface Polarization Model for the Dynamic Hysteresis of Perovskite Solar Cells, $J$. Phys. Chem. Lett. 8 (2017), 915. https://doi.org/10.1021/acs.jpclett.7b00045

11. K.T. Cho, G. Grancini, Y. Lee, E. Oveisi, J. Ryu, O. Almora, M. Tschumi, P.A. Schouwink, G. Seo, S. Heo, J. Park, J. Jang, S. Paek, G. Garcia-Belmonte, M.K. 
Nazeeruddin, Selective Growth of Layered Perovskites for Stable and Efficient $\begin{array}{llllll}\text { Photovoltaics, Energy Environ. Sci. } 11 & \text { (2018), } 952 .\end{array}$ https://doi.org/10.1039/C7EE03513F

12. A. Guerrero*, A. Bou, O. Almora, T. Heumuller, G. Garcia-Belmonte, J. Bisquert, Y. Hou*, C.J. Brabec*, Switching Off Hysteresis in Perovskite Solar Cells by Finetuning Energy Levels of Extraction Layers, Adv. Energy Mater. 8 (2018), 201703376. https://doi.org/10.1002/aenm.201703376

13. X. Tang*, G.J. Matt, S. Gao, E. Gu, O. Almora, C.J. Brabec*, Electrical-Field Driven Tunable Spectral Responses in a Broadband-Absorbing Perovskite Photodiode, ACS Appl. Mater. Interfaces $11 \quad$ (2019), 39018. https://doi.org/10.1021/acsami.9b14788

14. C. Zhang*, T. Heumueller, W. Gruber, O. Almora, X. Du, L. Ying, J. Chen, T. Unruh, Y. Cao, N. Li*, C.J. Brabec*, Comprehensive Investigation and Analysis of Bulk-heterojunction Microstructure of High-performance PCE11:PCBM Solar Cells, ACS Appl. Mater. Interfaces $11 \quad$ (2019), 18555. https://doi.org/10.1021/acsami.8b22539

15. L. Vaillant-Roca*, A. Peukert, M. Wittmer, O. Almora, A. Chanaewa, E. von Hauff, Temperature Dependent Seeding Effects on Hydrothermally Grown Zinc Oxide Nanorods: Towards Low Temperatures and High Scalability, Rev. Cubana Fis. 31 (2014), E85.

16. X. Du, T. Heumueller, W. Gruber, O. Almora, A. Classen, J. Qu, F. He, T. Unruh, N. Li, C.J. Brabec, Unraveling the Microstructure-Related Device Stability for Polymer Solar Cells Based on Nonfullerene Small Molecular Acceptors, Adv. Mater. (2020), 1908305. https://doi.org/10.1002/adma.201908305 